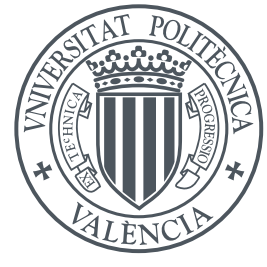

UNIVERSITAT
POLITECCNICA
DE VALĖNCIA

\title{
Global pseudodifferential operators in spaces of ultradifferentiable functions
}

Author:

Vicente Asensio López
Advisor:

David Jornet Casanova

Valencia, July 2021 

Odio gli indifferenti. Credo che vivere voglia dire essere partigiani. Antonio Gramsci 



\section{Acknowledgements}

Voldria començar aquesta secció donant les gràcies, per descomptat, al director de tesi David Jornet per tot l'esforç i ajuda, no només durant aquest període, sinó també des que vaig estudiar el màster. Et voldria donar les gràcies especialment per haver sentit que només tu em podies ajudar a resoldre els problemes que anaven sorgint. Crec que mai t'ho he dit, i em sembla un bon lloc per dir-t'ho.

Vull agrair especialment a José Bonet el seu recolçament constant i les seues bones paraules durant aquest temps. I també m'agradaria donar les gràcies a Carmen Fernández i Antonio Galbis per l'interés en els resultats de la tesi i els seus ànims. A tots us done les gràcies pels comentaris que han fet millorar la qualitat d'aquesta tesi.

Aquesta tesi ha estat desenvolupada a l'Institut Universitari de Matemàtica Pura i Aplicada. Voldria donar les gràcies als directors José Calabuig i Alfred Peris i als seus equips directius per la seua amabilitat i comprensió amb mi. Quisiera también agradecer a Minerva por su inestimable ayuda, a Nuria por sus consejos y comentarios, a Víctor por los buenos momentos, a Nazaret por los viajes, y a los compañeros y becarios que me han ofrecido su colaboración, ayuda, y, en definitiva, que han amenizado este proceso en la sala del café.

I am very thankful to Armin Rainer for accepting me to visit the Fakultät für Mathematik, and I am grateful to Gerhard Schindl for his predisposition to collaborate with me. Despite the time we faced, I enjoyed it a lot. As you once told me, I may tell my 'unusual visit in Vienna 2020' to my great- 
grandchildren within many years. I would like to thank all my colleagues, who created a great atmosphere in the faculty during that enjoyable time.

Vorrei ringraziare Chiara Boiti, non soltanto per la disponibilità e collaborazione ma sopratutto per le belle passeggiate della domenica. Mi piacerebbe inoltre esprimere la mia gratitudine ad Alessandro Oliaro per l'incoraggiamento e l'interese dimostratimi. Grazie davvero a tutti e due del prezioso aiuto.

Durante estos años he tenido la fortuna de impartir docencia en l'Escola Tècnica Superior d'Enginyeria Informàtica y en l'Escola Tècnica Superior d'Enginyeria Industrial. A todos los profesores de las escuelas les doy las gracias por su ayuda, y por echarme unos cuantos años menos de los que tengo. En especial, a Antonio Hervás y María Carmen Pedraza les agradezco su inestimable ayuda por ponerme las cosas tan fáciles. A Elena Alemany y a Carles Bivià su simpatía y su tenacidad durante este difícil último curso. A todos los compañeros de las asignaturas os doy, de corazón, las gracias por haberme hecho sentir uno más.

En darrer lloc, i no per això menys important, voldria tindre un record per als familiars i amics que m'han ajudat i animat durant aquest camí. No ha sigut fàcil. Moltes gràcies. 


\section{Summary}

In this thesis we study pseudodifferential operators, which are integral operators of the form

$$
f \mapsto \int_{\mathbb{R}^{d}}\left(\int_{\mathbb{R}^{d}} e^{i(x-y) \cdot \xi} a(x, y, \xi) f(y) d y\right) d \xi,
$$

in the global class of ultradifferentiable functions of Beurling type $\mathcal{S}_{\omega}\left(\mathbb{R}^{d}\right)$ as introduced by Björck, when the weight function $\omega$ is given in the sense of Braun, Meise, and Taylor.

We develop a symbolic calculus for these operators, treating also the change of quantization, the existence of pseudodifferential parametrices and applications to global wave front sets.

The thesis consists of four chapters. In Chapter 1 we introduce global symbols and amplitudes and show that the corresponding pseudodifferential operators are well defined and continuous in $\mathcal{S}_{\omega}\left(\mathbb{R}^{d}\right)$. These results are extended in Chapter 2 for arbitrary quantizations, which leads to the study of the transpose of any quantization of a pseudodifferential operator, and the composition of two different quantizations of pseudodifferential operators. In Chapter 3 we develop the method of the parametrix, providing sufficient conditions for the existence of left parametrices of a pseudodifferential operator, which motivates in Chapter 4 the definition of a new global wave front set for ultradistributions in $\mathcal{S}_{\omega}^{\prime}\left(\mathbb{R}^{d}\right)$ given in terms of Weyl quantizations. Then, we compare this wave front set with the Gabor wave front set defined by the STFT and give applications to the regularity of Weyl quantizations. 



\section{Resumen}

En esta tesis estudiamos operadores pseudodiferenciales, que son operadores integrales de la forma

$$
f \mapsto \int_{\mathbb{R}^{d}}\left(\int_{\mathbb{R}^{d}} e^{i(x-y) \cdot \xi} a(x, y, \xi) f(y) d y\right) d \xi,
$$

en las clases globales de funciones ultradiferenciables de tipo Beurling $\mathcal{S}_{\omega}\left(\mathbb{R}^{d}\right)$ introducidas por Björck, cuando la función peso $\omega$ viene dada en el sentido de Braun, Meise y Taylor.

Desarrollamos el cálculo simbólico para estos operadores, tratando además el cambio de cuantización, la existencia de paramétrix pseudodiferencial y aplicaciones al frente de ondas global.

La tesis consta de cuatro capítulos. En el Capítulo 1 introducimos los símbolos y amplitudes globales, y demostramos que los correspondientes operadores pseudodiferenciales están bien definidos y son continuos en $\mathcal{S}_{\omega}\left(\mathbb{R}^{d}\right)$. Estos resultados son extendidos en el Capítulo 2 para cuantizaciones arbitrarias, lo que conduce al estudio del traspuesto de cualquier cuantización de un operador pseudodiferencial y a la composición de dos cuantizaciones distintas de operadores pseudodiferenciales. En el Capítulo 3, desarrollamos el método de la paramétrix, dando condiciones suficientes para la existencia de paramétrix por la izquierda de un operador pseudodiferencial, que motiva en el Capítulo 4 la definición de un nuevo frente de ondas global para ultradistribuciones en $\mathcal{S}_{\omega}^{\prime}\left(\mathbb{R}^{d}\right)$ dada en términos de cuantizaciones de Weyl. Comparamos este frente 
de ondas con el frente de ondas de Gabor definido mediante la STFT y damos aplicaciones a la regularidad de las cuantizaciones de Weyl. 


\section{Resum}

En aquesta tesi estudiem operadors pseudodiferencials, que són operadors integrals de la forma

$$
f \mapsto \int_{\mathbb{R}^{d}}\left(\int_{\mathbb{R}^{d}} e^{i(x-y) \cdot \xi} a(x, y, \xi) f(y) d y\right) d \xi,
$$

en les classes globals de funcions ultradiferenciables de tipus Beurling $\mathcal{S}_{\omega}\left(\mathbb{R}^{d}\right)$ introduïdes per Björck, quan la funció pes $\omega$ ve donada en el sentit de Braun, Meise i Taylor.

Desenvolupem el càlcul simbòlic per aquestos operadors, tractant, a més a més, el canvi de quantització, l'existència de paramètrix pseudodiferencial i aplicacions al front d'ones global.

La tesi consisteix de quatre capítols. Al Capítol 1 introduïm els símbols i amplituds globals, i demostrem que els corresponents operadors pseudodiferencials estan ben definits i són continus en $\mathcal{S}_{\omega}\left(\mathbb{R}^{d}\right)$. Aquestos resultats són estesos al Capítol 2 per a quantitzacions arbitràries, que condueix a l'estudi del transposat de qualsevol quantització d'un operador pseudodiferencial i a la composició de dues quantitzacions distintes d'operadors pseudodiferencials. Al Capítol 3 desenvolupem el mètode de la paramètrix, donant condicions suficients per a l'existència de paramètrix per l'esquerra d'un operador pseudodiferencial donat, que motiva al Capítol 4 la definició d'un nou front d'ones global per a ultradistribucions en $\mathcal{S}_{\omega}^{\prime}\left(\mathbb{R}^{d}\right)$ mitjançant quantitzacions de Weyl. Comparem aquest front d'ones amb el front d'ones de Gabor definit mitjançant la STFT i donem aplicacions a la regularitat de les quantitzacions de Weyl. 



\section{Contents}

$\begin{array}{ll}\text { Introduction } & 1\end{array}$

0 Preliminaries $\quad 5$

0.1 Weight functions $\ldots \ldots \ldots \ldots \ldots \ldots \ldots \ldots$

0.2 Spaces of ultradifferentiable functions $\ldots \ldots \ldots \ldots \ldots$

0.3 Ultradifferential operators $\ldots \ldots \ldots \ldots \ldots$

0.4 Time-frequency analysis . . . . . . . . . . . . . . . . 24

1 Global pseudodifferential operators $\quad 29$

1.1 Symbols and amplitudes $\ldots \ldots \ldots \ldots . \ldots \ldots$

1.2 Continuity of the operator $\ldots \ldots \ldots \ldots \ldots$

2 Quantizations for pseudodifferential operators $\quad 59$

2.1 Symbolic calculus . . . . . . . . . . . . . . . . . . 60

2.2 Properties of formal sums . . . . . . . . . . . . . . . . . . 76

2.3 Behaviour of the kernel of a pseudodifferential operator . . . . . . . . 80 
2.4 The transposition and composition of operators . . . . . . . . . . 107

3 Parametrices

3.1 Global regularity . . . . . . . . . . . . . . . . . . . . . 116

3.2 Example .............................. 123

3.3 Global hypoellipticity . . . . . . . . . . . . . . . . . . 127

4 The Weyl wave front set

4.1 The $\omega$-wave front set . . . . . . . . . . . . . . . . . . . . . 137

4.2 The Weyl wave front set . . . . . . . . . . . . . . . . 159

4.3 Regularity of Weyl quantizations . . . . . . . . . . . . . . . . 171

Index

177

References

179

xiv 


\section{Introduction}

Pseudodifferential operators $(\Psi$ do $)$ are integral operators of the form

$$
f \mapsto \int_{\mathbb{R}^{d}} e^{i x \cdot \xi} p(x, \xi) \widehat{f}(\xi) d \xi,
$$

where $x \cdot \xi$ is the scalar product of the vectors $x$ and $\xi$ in $\mathbb{R}^{d}, f$ belongs to a local or global class of functions, $\widehat{f}$ is its Fourier transform and $p(x, \xi)$ is another function, called symbol, that satisfies the necessary properties to ensure that the operator is well defined and continuous when acting on the class of functions. Sometimes we need to use amplitudes $a(x, y, \xi)$ instead of symbols to understand the operator that, in this case, is written as an iterated integral given by

$$
f \mapsto \int_{\mathbb{R}^{d}}\left(\int_{\mathbb{R}^{d}} e^{i(x-y) \cdot \xi} a(x, y, \xi) f(y) d y\right) d \xi .
$$

Such operators generalize linear partial differential operators with variable coefficients and appear, among in many other applications (like topology, differential geometry, signal and image processing, etc.), initially when looking for an approximate solution (parametrix) of a differential equation given by an elliptic or hypoelliptic linear partial differential operator with variable coefficients. The local theory of pseudodifferential operators grew out of the study of singular integral operators, and it was developed after the systematic studies of Kohn and Nirenberg [48], and Hörmander [43], and others.

After that, the theory of $\Psi$ do has been widely developed in local Gevrey classes, which are spaces of (non-quasianalytic) ultradifferentiable functions 
in between real analytic and $C^{\infty}$ functions. The study of several problems in general classes of ultradifferentiable functions has received a lot of attention in the last 60 years. In the 80's, several authors (Hashimoto, Matsuzawa and Morimoto [41] and Iftimie [46]) gave different versions of Gevrey pseudodifferential operators of finite order, that is, given by symbols of moderate growth at infinity. Boutet de Monvel had studied a certain class of operators of infinite order, i.e. with symbols with exponential growth at infinity in some variables (and hence more general for applications). In 1985, Zanghirati [65] gave symbols of infinite order of Gevrey type; see the monograph Rodino [60] for an excellent introduction to this topic. In all these cases the spaces of functions considered are of Roumieu type (the topological structure of the spaces looks like that of the space of real-analytic funtions). Motivated by these results, Fernández, Galbis, and Jornet [33] developed a full theory of pseudodifferential operators of infinite order in the variable $\xi$ of the symbol (or amplitude) with the corresponding symbolic calculus on classes of ultradifferentiable functions of Beurling type (the topological structure looks like the one of the space of all smooth functions) in the sense of Braun, Meise, and Taylor [20].

The classic theory of $\Psi$ do, as well as all the mentioned works, is of local type. That is, it is based on the study of the solutions of the operators in a small enough neighbourhood of a given point. In [32] the authors give sufficient conditions to construct parametrices, i.e. approximate inverses, of the symbols introduced in [33]. The existence of a left parametrix for the symbol gives hypoellipticity in the corresponding class of functions for the $\Psi$ do. Hence, the possible ultradistribution solutions of the operator when the datum is an ultradifferentiable function are, in fact, as regular as the datum.

More recently, several authors have studied $\Psi$ do and Fourier integral operators of infinite order in spaces of Gelfand-Shilov type, which are global classes of ultradifferentiable functions with estimates in terms of the derivatives or of the Fourier transform of Gevrey type, see for instance [22, 23, 24, 27]. In a more general setting, Prangoski [58] introduced $\Psi$ do given by symbols of infinite order in all the variables for ultradifferentiable classes defined in the sense of Komatsu (with sequences), inspired by the classical global theory of $\Psi$ do in the Schwartz class that can be found in the book of Shubin [64]. Prangoski uses some kind of entire functions with prescribed exponential growth, which is crucial to understand the operators using integration by parts. Later, Cappiello, Pilipović, and Prangoski [25] gave sufficient conditions on the symbols to construct parametrices for the operators in [58].

In the theory of partial differential equations, the wave front set locates the singularities of a distribution and, at the same time, describes the directions 
of the high frequencies (in terms of the Fourier transform) responsible for those singularities. In the classical context of Schwartz distributions theory, it was originally defined by Hörmander [44]. In global classes of functions and distributions, the concept of singular support does not make sense since we need the information on the whole $\mathbb{R}^{d}$. However, we can still define a global wave front set to describe the micro-regularity of a distribution.

Very recently, Rodino and Wahlberg [61] recovered the concept of the $C^{\infty}$ global wave front set of [45] for the context of tempered distributions, showing that it can be reformulated in terms of the short-time Fourier transform. It is very natural to use methods of time-frequency analysis in connection with the wave front set, as the wave front set treats simultaneously analysis of points (the variables) and directions (the covariables). The authors prove also in [61] that this wave front set can be described merely by a Gabor frame, i.e. with the information of the decay of the Gabor coefficients in a sufficiently dense lattice.

Boiti, Jornet, and Oliaro [14] presented the ultradifferentiable version of the analytic wave front set found in $[26,45,61]$ in the Beurling setting for $\omega$ ultradistributions, where $\omega$ is a subadditive weight function in the sense of [20], showing that it can be described also in terms of Gabor frames, and applying it to the study of global regularity of pseudodifferential operators of infinite order.

The aim of this thesis is to introduce and study pseudodifferential operators in classes of global ultradifferentiable functions of Beurling type $\mathcal{S}_{\omega}\left(\mathbb{R}^{d}\right)$ (as the ones defined by [8] in the sense of [20]), using tools and techniques from time-frequency analysis and Fourier analysis, and to provide applications to global wave front sets in this setting.

In Chapter 1, we introduce global pseudodifferential operators in $\mathcal{S}_{\omega}\left(\mathbb{R}^{d}\right)$ by means of oscillatory integrals for global amplitudes. It turns out that the action of a pseudodifferential operator on a function in $\mathcal{S}_{\omega}\left(\mathbb{R}^{d}\right)$ can be written as an iterated integral, and we will show that this action is linear and continuous from $\mathcal{S}_{\omega}\left(\mathbb{R}^{d}\right)$ into $\mathcal{S}_{\omega}\left(\mathbb{R}^{d}\right)$. Moreover, this operator will be extended linearly and continuously to an operator from $\mathcal{S}_{\omega}^{\prime}\left(\mathbb{R}^{d}\right)$ into $\mathcal{S}_{\omega}^{\prime}\left(\mathbb{R}^{d}\right)$. We extend these results for arbitrary quantizations in Chapter 2. Furthermore, we develop a symbolic calculus, also valid for quantizations, necessary for the study of the composition of two given pseudodifferential operators. Chapter 3 is devoted to the construction of a suitable parametrix for the pseudodifferential operators considered. Finally, in Chapter 4 we define the analogous global wave front set to the one given in $[45,61]$ for the ultradifferentiable setting using 
Weyl quantizations, completing the results started in [14]. Finally, we give applications to the regularity of pseudodifferential operators. 


\section{Chapter 0}

\section{Preliminaries}

We detail the necessary preliminaries for the following chapters. In particular, we introduce the notation on multi-indices we use.

Let $\mathbb{N}_{0}=\mathbb{N} \cup\{0\}=\{0,1,2, \ldots\}$. In the following $\alpha$ stands for $\left(\alpha_{1}, \ldots, \alpha_{d}\right) \in$ $\mathbb{N}_{0}^{d}$, a multi-index of dimension $d$. We denote the length of $\alpha$ by

$$
|\alpha|=\alpha_{1}+\cdots+\alpha_{d} .
$$

For two multi-indices $\alpha$ and $\beta$, we denote $\beta \leq \alpha$ for $\beta_{j} \leq \alpha_{j}, j=1, \ldots, d$. Moreover,

$$
\alpha !=\alpha_{1} ! \cdots \alpha_{d} !
$$

and if $\beta \leq \alpha$, then

$$
\left(\begin{array}{l}
\alpha \\
\beta
\end{array}\right)=\frac{\alpha !}{\beta !(\alpha-\beta) !} .
$$

For $x=\left(x_{1}, \ldots, x_{d}\right) \in \mathbb{R}^{d}$, we put

$$
x^{\alpha}=x_{1}^{\alpha_{1}} \cdots x_{d}^{\alpha_{d}},
$$

and if $\xi=\left(\xi_{1}, \ldots, \xi_{d}\right) \in \mathbb{R}^{d}, x \cdot \xi$ is the scalar product, and is equal to $x_{1} \xi_{1}+$ $\cdots+x_{d} \xi_{d}$. We denote

$$
\langle x\rangle=\left(1+|x|^{2}\right)^{1 / 2}, \quad x \in \mathbb{R}^{d},
$$


where $|x|$ is the Euclidean norm of $x$. We write

$$
\partial^{\alpha}=\left(\frac{\partial}{\partial x_{1}}\right)^{\alpha_{1}} \cdots\left(\frac{\partial}{\partial x_{d}}\right)^{\alpha_{d}}
$$

and using the notation

$$
D_{x_{j}}=-i \frac{\partial}{\partial x_{j}}, \quad j=1, \ldots, d,
$$

where $i$ is the imaginary unit, we denote

$$
D^{\alpha}=D_{x}^{\alpha}=D_{x_{1}}^{\alpha_{1}} \cdots D_{x_{d}}^{\alpha_{d}}
$$

The well-known inequalities collected in the next lemma will be frequently used during the text.

Lemma 0.1. Let $\alpha, \beta \in \mathbb{N}_{0}^{d}, N \in \mathbb{N}$, and $m, n, r \in \mathbb{N}_{0}$. Then

(1) $\sum_{|\alpha|=N} \frac{N !}{\alpha !}=d^{N}$. In particular, $\sum_{|\alpha|=N} 1 \leq d^{N}$.

(2) $\alpha ! \leq|\alpha| ! \leq d^{|\alpha|} \alpha !$

(3) $\sum_{\beta \leq \alpha}\left(\begin{array}{l}\alpha \\ \beta\end{array}\right)=2^{|\alpha|}$. In particular, if $\beta \leq \alpha$, then $\left(\begin{array}{c}\alpha \\ \beta\end{array}\right) \leq 2^{|\alpha|}$.

(4) $\sum_{\alpha_{1}+\cdots+\alpha_{N}=\alpha} \frac{\alpha !}{\alpha_{1} ! \cdots \alpha_{N} !}=N^{|\alpha|}$, where $\alpha_{1}, \ldots, \alpha_{N} \in \mathbb{N}_{0}^{d}$.

(5) (Vandermonde's Identity). $\sum_{k=0}^{r}\left(\begin{array}{c}m \\ k\end{array}\right)\left(\begin{array}{c}n \\ r-k\end{array}\right)=\left(\begin{array}{c}n+m \\ r\end{array}\right)$.

(6) If $\beta \leq \alpha$, then $\left(\begin{array}{l}\alpha \\ \beta\end{array}\right) \leq\left(\begin{array}{l}|\alpha| \\ |\beta|\end{array}\right)$. In particular, $\frac{|\beta| !}{\beta !} \leq \frac{|\alpha| \text { ! }}{\alpha !}$.

(7) For all $j \in \mathbb{N}_{0},\left|\left\{\alpha \in \mathbb{N}_{0}^{d}:|\alpha|=j\right\}\right|=\left(\begin{array}{c}j+d-1 \\ d-1\end{array}\right)$.

(8) The series $\sum_{\alpha \in \mathbb{N}_{0}^{d}} e^{-|\alpha|}$ is convergent. 
Proof. (1) - (3) are standard properties of multi-indices (see for example [60, $(1.2 .2)-(1.2 .7)])$. The proof of (4) follows proceeding as in (3). Formula (5) is shown in [7, Identity 132], and with this, we can show (6). Formula (7) is [55, (0.3.16)], and then, we obtain (8):

$$
\sum_{\alpha \in \mathbb{N}_{0}^{d}} e^{-|\alpha|}=\sum_{j=0}^{\infty} \sum_{|\alpha|=j} e^{-j}=\sum_{j=0}^{\infty} e^{-j}\left(\begin{array}{c}
j+d-1 \\
d-1
\end{array}\right) \leq 2^{d-1} \sum_{j=0}^{\infty}(2 / e)^{j}<+\infty
$$

The following proof is taken from [28, Lemma 2.6.2].

Lemma 0.2 (Peetre's inequality). For all $t \in \mathbb{R}$ and $x, y \in \mathbb{R}^{d}$, we have

$$
\langle x\rangle^{t} \leq \sqrt{2}^{|t|}\langle x-y\rangle^{|t|}\langle y\rangle^{t} .
$$

Proof. Since $(|x|-|y|)^{2} \geq 0$, we have $2|x||y| \leq|x|^{2}+|y|^{2}$. Then,

$$
1+|x+y|^{2} \leq 1+(|x|+|y|)^{2} \leq 1+2|x|^{2}+2|y|^{2} \leq 2\left(1+|x|^{2}\right)\left(1+|y|^{2}\right),
$$

and therefore $\langle x+y\rangle \leq \sqrt{2}\langle x\rangle\langle y\rangle, x, y \in \mathbb{R}^{d}$. From this, we deduce the result for $t \geq 0$ if $x+y$ is replaced by $x$. For $t<0$, replace $x+y$ by $y$.

\subsection{Weight functions}

In our setting, we work with weight functions as the ones defined by Braun, Meise, and Taylor [20].

Definition 0.3. $A$ non-quasianalytic weight function $\omega:[0,+\infty[\rightarrow[0,+\infty[$ is a continuous and increasing function which satisfies:

( $\alpha)$ There exists $L \geq 1$ such that $\omega(2 t) \leq L(\omega(t)+1), t \geq 0$;

( $\beta) \int_{1}^{+\infty} \frac{\omega(t)}{t^{2}} d t<+\infty$

$(\gamma) \log (t)=o(\omega(t))$ as $t \rightarrow \infty$

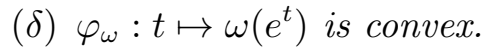


We extend the weight function to $\mathbb{C}^{d}$ in a radial way: $\omega(z)=\omega(|z|), z \in \mathbb{C}^{d}$, where $|z|$ denotes the Euclidean norm in $\mathbb{C}^{d}$.

Condition $(\alpha)$ is weaker than the subadditivity. Indeed, as in [20, Lemma 1.2], we have, for $x, y \in \mathbb{R}^{d}$, where $L \geq 1$ is the constant appearing in Definition $0.3(\alpha)$,

$\omega(x+y) \leq \omega(2 \max \{|x|,|y|\}) \leq L \omega(\max \{|x|,|y|\})+L \leq L(\omega(x)+\omega(y)+1)$.

In [57, Proposition 1.1] it is proved that a weight function is (equivalent to) a subadditive weight function if and only if satisfies

$\left(\alpha_{0}\right)$ There exist $C>0, t_{0}>0$ : for all $\lambda \geq 1 \omega(\lambda t) \leq \lambda C \omega(t), \quad t \geq t_{0}$.

See $[21,31,56,57,59]$ for results involving property $\left(\alpha_{0}\right)$.

As a consequence of (0.1), since $\omega$ is an increasing function, we have

$$
\omega\left(\frac{x+y}{2}\right) \leq \omega(\max \{|x|,|y|\}) \leq \omega(x)+\omega(y), \quad x, y \in \mathbb{R}^{d} .
$$

Moreover, since $|(x, y)| \leq|x|+|y|$, we also have

$$
\omega(x, y) \leq \omega(|x|+|y|) \leq L \omega(x)+L \omega(y)+L, \quad x, y \in \mathbb{R}^{d},
$$

and we can see, for all $x, y, \xi \in \mathbb{R}^{d}$,

$$
\omega(x, y, \xi) \leq \omega(\sqrt{3} \max \{|x|,|y|,|\xi|\}) \leq L \omega(x)+L \omega(y)+L \omega(\xi)+L .
$$

On the other hand, let $q \in \mathbb{N}_{0}$ be such that $d \leq 2^{q}$. Then, using $q$ times property $(\alpha)$,

$$
\omega(d x) \leq L^{q} \omega\left(\left(d / 2^{q}\right) x\right)+L^{q}+\cdots+L \leq L^{q} \omega(x)+L^{q}+\cdots+L, \quad x \in \mathbb{R}^{d} .
$$

Hence, for $L^{\prime}:=L^{q}+\cdots+L \geq 1$, which depends on $L$ and on $d$,

$$
\omega(x) \leq \omega\left(\left|x_{1}\right|+\cdots+\left|x_{d}\right|\right) \leq \omega\left(d|x|_{\infty}\right) \leq L^{\prime} \omega\left(|x|_{\infty}\right)+L^{\prime} \leq L^{\prime} \omega(x)+L^{\prime}
$$

for all $x=\left(x_{1}, \ldots, x_{d}\right) \in \mathbb{R}^{d}$, where $|x|_{\infty}$ is the supremum norm in $\mathbb{R}^{d}$.

It is known that property $(\beta)$ of the weight $\omega$, called non-quasianalyticity condition, implies $\omega(t)=o(t)$ when $t \rightarrow \infty$ :

$$
0 \leq \frac{\omega(t)}{t}=\int_{t}^{+\infty} \frac{\omega(t)}{s^{2}} d s \leq \int_{t}^{+\infty} \frac{\omega(s)}{s^{2}} d s .
$$

We consider now property $(\delta)$ of Definition 0.3 and introduce: 
Definition 0.4. Given $\omega$ a weight function, the Young conjugate $\varphi_{\omega}^{*}:[0, \infty[\rightarrow$ $\left[0, \infty\left[\right.\right.$ of $\varphi_{\omega}$ is defined by

$$
\varphi_{\omega}^{*}(t):=\sup _{s \geq 0}\left\{s t-\varphi_{\omega}(s)\right\}
$$

When the weight chosen is clear, we will write $\varphi_{\omega}$ and $\varphi_{\omega}^{*}$ simply $\varphi$ and $\varphi^{*}$. We will assume without loss of generality that $\left.\omega\right|_{[0,1]} \equiv 0$, which gives some useful properties (see [20]). In fact, $\varphi^{*}(0)=0$, and because of the convexity of $\varphi^{*}$, the function $\varphi^{*}(t) / t$ is increasing and $\left(\varphi^{*}\right)^{*}=\varphi$. Moreover, we have by $(0.1)$,

$$
\omega(\langle x\rangle) \leq \omega(1+|x|) \leq L \omega(x)+L, \quad x \in \mathbb{R}^{d}
$$

Example 0.5. [20, Example 4.3] The following functions are, after a change in some interval $[0, M]$, examples of weight functions:

(i) Gevrey weights: $\omega(t)=t^{p}, 0<p<1$.

(ii) $\omega(t)=(\log (1+t))^{s}, s>1$.

(iii) $\omega(t)=t^{p}(\log (e+t))^{s}, 0<p<1, s \neq 0$.

The following inequalities will be used throughout the next chapters. For their proof, see [33, Lemma 1.4] and [15, Appendix A].

Lemma 0.6. For every $\lambda>0, k \in \mathbb{N}, t \geq 1$,

$$
\begin{aligned}
t^{k} & \leq e^{\lambda \varphi^{*}\left(\frac{k}{\lambda}\right)} e^{\lambda \omega(t)} ; \\
\inf _{j \in \mathbb{N}_{0}} t^{-j} e^{k \varphi^{*}\left(\frac{j}{k}\right)} & \leq e^{-k \omega(t)+\log (t)} ; \\
\inf _{j \in \mathbb{N}_{0}} t^{-2 j} e^{k \varphi^{*}\left(\frac{2 j}{k}\right)} & \leq C e^{-(k-1) \omega(t)},
\end{aligned}
$$

for some $C>0$, independent of $k \in \mathbb{N}$.

It is possible to improve (0.8) when that infimum is attained in a finite set as this result shows (see [33, Lemma 1.5]):

Lemma 0.7. If $\frac{k}{N} \varphi^{*}\left(\frac{N}{k}\right) \leq \log (t) \leq \frac{k}{N+1} \varphi^{*}\left(\frac{N+1}{k}\right)$, then

$$
t^{-N} e^{2 k \varphi^{*}\left(\frac{N}{2 k}\right)} \leq e^{-k \omega(t)+\log (t)}
$$


By condition $(\alpha)$ in Definition 0.3, there exists $L \geq 1$ such that $\omega($ et $) \leq$ $L \omega(t)+L$. By abuse of notation, $L \geq 1$ will be this constant in the following. Under this assumption we have the following results from [20]. For a detailed proof of them we refer to [15, Appendix A] (cf. [42, Remark 2.8(c)]).

Lemma 0.8. (i) We have

$$
\lambda L^{k} \varphi^{*}\left(\frac{x}{\lambda L^{k}}\right)+k x \leq \lambda \varphi^{*}\left(\frac{x}{\lambda}\right)+\lambda \sum_{j=1}^{k} L^{j}
$$

for every $x \geq 0, \lambda>0$ and $k \in \mathbb{N}$.

(ii) For all $s, t, \lambda>0$,

$$
2 \lambda \varphi^{*}\left(\frac{s+t}{2 \lambda}\right) \leq \lambda \varphi^{*}\left(\frac{s}{\lambda}\right)+\lambda \varphi^{*}\left(\frac{t}{\lambda}\right) \leq \lambda \varphi^{*}\left(\frac{s+t}{\lambda}\right) .
$$

(iii) For every $\lambda>0$ and $B>0$ there exists $C>0$ such that

$$
B^{|\alpha|} \alpha ! \leq C e^{\lambda \varphi^{*}\left(\frac{|\alpha|}{\lambda}\right)}, \quad \alpha \in \mathbb{N}_{0}^{d} .
$$

Formula (0.12) can be improved by assuming a further condition on the weight function $\omega$ :

Lemma 0.9. Let $\omega$ be a weight function such that $\omega(t)=o\left(t^{a}\right), t \rightarrow \infty$, for some $0<a \leq 1$. Then, for every $\lambda>0$ and $B>0$ there exists $C>0$ such that

$$
B^{|\alpha|} \alpha ! \leq C e^{a \lambda \varphi^{*}\left(\frac{|\alpha|}{\lambda}\right)}, \quad \alpha \in \mathbb{N}_{0}^{d} .
$$

When considering a suitable change of weights, the following result is useful to estimate their Young conjugate. We observe that if $\omega \leq \sigma$, it is obvious that $\varphi_{\sigma}^{*} \leq \varphi_{\omega}^{*}$.

Lemma 0.10. Let $0<a \leq 1$ and let $\omega$ and $\sigma$ be weight functions. Then:

(1) If $\omega\left(t^{1 / a}\right)=o(\sigma(t))$ as $t \rightarrow \infty$, for all $\lambda, \mu>0$ there exists $C_{\lambda, \mu}>0$ such that

$$
\lambda \varphi_{\sigma}^{*}\left(\frac{j}{\lambda}\right) \leq C_{\lambda, \mu}+a \mu \varphi_{\omega}^{*}\left(\frac{j}{\mu}\right), \quad j \in \mathbb{N}_{0} .
$$

(2) If $\omega\left(t^{1 / a}\right)=O(\sigma(t))$ as $t \rightarrow \infty$, there is $C>0$ so that for each $\lambda>0$,

$$
\lambda \varphi_{\sigma}^{*}\left(\frac{j}{\lambda}\right) \leq \lambda+a \frac{\lambda}{C} \varphi_{\omega}^{*}\left(\frac{j C}{\lambda}\right), \quad j \in \mathbb{N}_{0} .
$$


Proof. (1) By assumption, for all $\lambda, \mu>0$ there exists $C_{\lambda, \mu}>0$ such that

$$
\mu \omega\left(t^{1 / a}\right) \leq C_{\lambda, \mu}+\lambda \sigma(t), \quad t \geq 0 .
$$

Then, for $j \in \mathbb{N}_{0}$, since $0<a \leq 1$,

$$
\begin{aligned}
\lambda \varphi_{\sigma}^{*}\left(\frac{j}{\lambda}\right) & =\sup _{s \geq 0}\left\{s j-\lambda \sigma\left(e^{s}\right)\right\} \\
& \leq C_{\lambda, \mu}+\sup _{s \geq 0}\left\{s j-\mu \omega\left(e^{s / a}\right)\right\} \\
& \leq C_{\lambda, \mu}+a \mu \sup _{s \geq 0}\left\{\frac{s}{a} \frac{j}{\mu}-\omega\left(e^{s / a}\right)\right\} \\
& =C_{\lambda, \mu}+a \mu \sup _{t \geq 0}\left\{t \frac{j}{\mu}-\omega\left(e^{t}\right)\right\}=C_{\lambda, \mu}+a \mu \varphi_{\omega}^{*}\left(\frac{j}{\mu}\right) .
\end{aligned}
$$

(2) There exists $C>0$ such that $\omega\left(t^{1 / a}\right) \leq C+C \sigma(t)$, so $-\sigma(t) \leq 1-$ $C^{-1} \omega\left(t^{1 / a}\right)$ for all $t \geq 0$. Therefore, for all $\lambda>0, j \in \mathbb{N}_{0}$, we obtain

$$
\begin{aligned}
\lambda \varphi_{\sigma}^{*}\left(\frac{j}{\lambda}\right) & =\lambda \sup _{s \geq 0}\left\{s \frac{j}{\lambda}-\sigma\left(e^{s}\right)\right\} \\
& \leq \lambda+a \frac{\lambda}{C} \sup _{s \geq 0}\left\{\frac{s}{a} \frac{j C}{\lambda}-\frac{1}{a} \omega\left(e^{s / a}\right)\right\} \\
& \leq \lambda+a \frac{\lambda}{C} \sup _{t \geq 0}\left\{t \frac{j C}{\lambda}-\omega\left(e^{t}\right)\right\}=\lambda+a \frac{\lambda}{C} \varphi_{\omega}^{*}\left(\frac{j C}{\lambda}\right) .
\end{aligned}
$$

We write $P(\xi, r)$ for the polydisc of center $\xi=\left(\xi_{1}, \ldots, \xi_{d}\right) \in \mathbb{C}^{d}$ and polyradius $r=\left(r_{1}, \ldots, r_{d}\right)$, where $r_{j}>0, j=1, \ldots, d$. That is,

$$
P(\xi, r)=\left\{\left(z_{1}, \ldots, z_{d}\right) \in \mathbb{C}^{d}:\left|z_{j}-\xi_{j}\right|<r_{j}, j=1, \ldots, d\right\} .
$$

Also,

$$
\partial P(\xi, r)=\left\{\left(z_{1}, \ldots, z_{d}\right) \in \mathbb{C}^{d}:\left|z_{j}-\xi_{j}\right|=r_{j}, j=1, \ldots, d\right\} .
$$

By Cauchy's Integral Formula for the derivatives (see for instance [62, Chapter 1.3]), we obtain:

Proposition 0.11 (Cauchy's inequalities). Let $\Omega \subset \mathbb{C}^{d}$ be an open set, $\xi \in \Omega$ and $r=\left(r_{1}, \ldots, r_{d}\right) \in \mathbb{R}^{d}, r_{j}>0, j=1, \ldots, d$ so that $\overline{P(\xi, r)} \subset \Omega$. If $f: \Omega \rightarrow \mathbb{C}$ is continuous and partially holomorphic, then

$$
\left|D^{\alpha} f(\xi)\right| \leq \frac{\alpha !}{r^{\alpha}} \sup _{z \in \partial P(\xi, r)}|f(z)|, \quad \alpha \in \mathbb{N}_{0}^{d}, \xi \in \Omega .
$$




\subsection{Spaces of ultradifferentiable functions}

We introduce the spaces of ultradifferentiable functions in the sense of [20] in terms of the Young conjugate.

Definition 0.12. Let $\omega$ be a weight function. For an open set $\Omega \subset \mathbb{R}^{d}$, we denote

$$
\mathcal{E}_{(\omega)}(\Omega):=\left\{f \in C^{\infty}\left(\mathbb{R}^{d}\right):|f|_{K, \lambda}<+\infty, \quad \forall K \subset \subset \Omega, \lambda>0\right\},
$$

and

$$
\mathcal{E}_{\{\omega\}}(\Omega):=\left\{f \in C^{\infty}\left(\mathbb{R}^{d}\right): \forall K \subset \subset \Omega, \exists \lambda>0 \text { such that }|f|_{K, \lambda}<+\infty\right\},
$$

where

$$
|f|_{K, \lambda}:=\sup _{\alpha \in \mathbb{N}_{0}^{d} x \in K} \sup _{x \in K}\left|D^{\alpha} f(x)\right| e^{-\lambda \varphi^{*}\left(\frac{|\alpha|}{\lambda}\right)} .
$$

The first space is endowed with the Fréchet topology given by the sequence of seminorms $|f|_{K_{n}, n}$, where $\left(K_{n}\right)_{n}$ is any compact exhaustion of $\Omega, n \in \mathbb{N}$. This is called the space of $\omega$-ultradifferentiable functions of Beurling type in $\Omega$. The second space is called the space of $\omega$-ultradifferentiable functions of Roumieu type in $\Omega$.

For a Gevrey weight $\omega(t)=t^{p}, 0<p<1$, the space $\mathcal{E}_{\{\omega\}}(\Omega)$ is the Gevrey class with exponent $1 / p$ (see e.g. [60, Definition 1.4.1] for the definition of the space).

We write $*$ for $(\omega)$ or $\{\omega\}$. For a compact set $K \subset \subset \Omega$, we denote by $\mathcal{D}_{*}(K):=\mathcal{E}_{*}(\Omega) \cap \mathcal{D}(K)$ and we define the spaces of test functions of Beurling and Roumieu type in $\Omega$ as

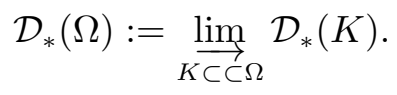

It is important to remark that these spaces are non-trivial if and only if condition $(\beta)$ is satisfied (see [20, Corollary 2.6] and [8, Lemma 1.3.10]). For further information on these spaces, see e.g. [20, Corollary 3.6, Proposition 3.9].

The elements in $\mathcal{D}_{(\omega)}^{\prime}(\Omega)$ are called $\omega$-ultradistributions of Beurling type in $\Omega$, and $\mathcal{D}_{\{\omega\}}^{\prime}(\Omega)$ is the space of $\omega$-ultradistributions of Roumieu type in $\Omega$. By [20, Proposition 3.9], $\mathcal{D}_{(\omega)}(\Omega) \subset \mathcal{D}_{\{\omega\}}(\Omega)$ with dense and continuous inclusion, therefore we consider $\mathcal{D}_{\{\omega\}}^{\prime}(\Omega)$ as a subspace of $\mathcal{D}_{(\omega)}^{\prime}(\Omega)$. Moreover, if 
$\sigma(t)=o(\omega(t))$ as $t \rightarrow \infty$, then $\mathcal{D}_{\{\omega\}}(\Omega) \subset \mathcal{D}_{(\sigma)}(\Omega)$ with dense and continuous inclusion.

For $T \in \mathcal{D}_{*}^{\prime}(\Omega)$, the support of $T$ is defined by

$$
\operatorname{supp}(T):=\left\{x \in \Omega: \forall U \text { neighbourhood of } x, \exists \varphi \in \mathcal{D}_{*}(U) \text { with }\langle T, \varphi\rangle \neq 0\right\} .
$$

The space of ultradistributions with compact support of Beurling and Roumieu type in $\Omega$ is denoted by $\mathcal{E}_{*}^{\prime}(\Omega)$.

We deal with spaces of global $\omega$-ultradifferentiable functions as the ones introduced by Björck [8]. First, we recall the definition of Fourier transform of $f \in L^{1}\left(\mathbb{R}^{d}\right)$ :

$$
\widehat{f}(\xi)=\mathcal{F} f(\xi):=\int_{\mathbb{R}^{d}} e^{-i x \cdot \xi} f(x) d x, \quad \xi \in \mathbb{R}^{d},
$$

with standard extensions to more general spaces of functions and distributions. The partial Fourier transform of $f \in L^{1}\left(\mathbb{R}^{2 d}\right)$ is defined by

$$
\mathcal{F}_{y \mapsto \xi} f(x, \xi):=\int_{\mathbb{R}^{d}} e^{-i y \cdot \xi} f(x, y) d y, \quad x, \xi \in \mathbb{R}^{d} .
$$

Definition 0.13. Given a weight function $\omega$, the space $\mathcal{S}_{\omega}\left(\mathbb{R}^{d}\right)$ is the space of all $f \in L^{1}\left(\mathbb{R}^{d}\right)$ such that $\left(f, \widehat{f} \in C^{\infty}\left(\mathbb{R}^{d}\right)\right.$ and) for all $\lambda>0$ and all multi-index $\alpha \in \mathbb{N}_{0}^{d}$,

$$
\sup _{x \in \mathbb{R}^{d}}\left|D^{\alpha} f(x)\right| e^{\lambda \omega(x)}<+\infty, \quad \sup _{\xi \in \mathbb{R}^{d}}\left|D^{\alpha} \widehat{f}(\xi)\right| e^{\lambda \omega(\xi)}<+\infty .
$$

These estimates form a fundamental system of seminorms for $\mathcal{S}_{\omega}\left(\mathbb{R}^{d}\right)$. It is a Fréchet space endowed with the topology generated by the seminorms given in Definition 0.13. By [8, Proposition 1.8.2], it is contained in the Schwartz class $\mathcal{S}\left(\mathbb{R}^{d}\right)$ and coincides with $\mathcal{S}\left(\mathbb{R}^{d}\right)$ when $\omega(t)=\log (1+t)$. Moreover, the Fourier transform is an automorphism in $\mathcal{S}_{\omega}\left(\mathbb{R}^{d}\right)$ and the space $\mathcal{S}_{\omega}\left(\mathbb{R}^{2 d}\right)$ is invariant under partial Fourier transform (see [13, Remark 4.10]).

Remark 0.14. [8, Proposition 1.8.6, Theorem 1.8.7] For any weight function $\omega$, we have $\mathcal{D}_{(\omega)}\left(\mathbb{R}^{d}\right) \subseteq \mathcal{S}_{\omega}\left(\mathbb{R}^{d}\right) \subseteq \mathcal{E}_{(\omega)}\left(\mathbb{R}^{d}\right)$ with continuous inclusion and $\mathcal{D}_{(\omega)}\left(\mathbb{R}^{d}\right)$ is dense in $\mathcal{S}_{\omega}\left(\mathbb{R}^{\bar{d}}\right)$.

The space $\mathcal{S}_{\omega}\left(\mathbb{R}^{d}\right)$ is nuclear for every weight function $\omega$. See, for instance, Boiti, Jornet, Oliaro, and Schindl [16, 17].

The following characterization will be useful throughout the thesis. 
Lemma 0.15. If $f \in \mathcal{S}\left(\mathbb{R}^{d}\right)$, then $f \in \mathcal{S}_{\omega}\left(\mathbb{R}^{d}\right)$ if and only if for all $\lambda, \mu>0$ there exists $C_{\lambda, \mu}>0$ such that

$$
\left|D^{\alpha} f(x)\right| \leq C_{\lambda, \mu} e^{\lambda \varphi^{*}\left(\frac{|\alpha|}{\lambda}\right)} e^{-\mu \omega(x)}, \quad \alpha \in \mathbb{N}_{0}^{d}, x \in \mathbb{R}^{d} .
$$

Proof. If $f \in \mathcal{S}_{\omega}\left(\mathbb{R}^{d}\right)$, then by [13, Theorem 4.8(5)], we have that for all $\lambda, \mu>0$ there exists $C_{\lambda, \mu}>0$ such that

$$
\sup _{x \in \mathbb{R}^{d}}\left|x^{\beta} D^{\alpha} f(x)\right| \leq C_{\lambda, \mu} e^{\lambda \varphi^{*}\left(\frac{|\alpha|}{\lambda}\right)} e^{\mu \varphi^{*}\left(\frac{|\beta|}{\mu}\right)}, \quad \alpha, \beta \in \mathbb{N}_{0}^{d} .
$$

We fix $\beta=\left(\beta_{1}, \ldots, \beta_{d}\right) \in \mathbb{N}_{0}^{d}$ and $x=\left(x_{1}, \ldots, x_{d}\right) \in \mathbb{R}^{d}$. We assume without losing generality $\left|x_{1}\right|=|x|_{\infty}$.

If $\left|x_{1}\right| \leq 1$, then $|x| \leq \sqrt{d}$, so for every $\mu>0$ we have $C_{\mu}=\sup _{\left|x_{1}\right| \leq 1} e^{\mu \omega(x)}>0$, and therefore for all $\mu>0$,

$$
\left|x_{1}\right|^{\beta_{1}+\cdots+\beta_{d}} \leq 1 \leq C_{\mu} e^{-\mu \omega(x)} .
$$

Since by (0.13) we have that for all $\lambda>0$ there exists $C_{\lambda}>0$ such that

$$
\left|D^{\alpha} f(x)\right| \leq \sup _{x \in \mathbb{R}^{d}}\left|D^{\alpha} f(x)\right| \leq C_{\lambda} e^{\lambda \varphi^{*}\left(\frac{|\alpha|}{\lambda}\right)}, \quad \alpha \in \mathbb{N}_{0}^{d},
$$

then,

$$
\left|x_{1}\right|^{\beta_{1}+\cdots+\beta_{d}}\left|D^{\alpha} f(x)\right| \leq 1 \cdot\left|D^{\alpha} f(x)\right| \leq C_{\lambda} C_{\mu} e^{\lambda \varphi^{*}\left(\frac{|\alpha|}{\lambda}\right)} e^{-\mu \omega(x)},
$$

and the result follows for $C_{\lambda, \mu}:=C_{\lambda} C_{\mu}>0$.

Now, we assume $\left|x_{1}\right|>1$. We have

$$
\left|x^{\beta} D^{\alpha} f(x)\right|=\left|x_{1}\right|^{\beta_{1}} \cdots\left|x_{d}\right|^{\beta_{d}}\left|D^{\alpha} f(x)\right| \leq\left|x_{1}\right|^{\beta_{1}+\cdots+\beta_{d}}\left|D^{\alpha} f(x)\right|=\left|x^{\gamma} D^{\alpha} f(x)\right|,
$$

where $\gamma=\left(\beta_{1}+\cdots+\beta_{d}, 0, \ldots, 0\right) \in \mathbb{N}_{0}^{d}$, satisfying $|\gamma|=|\beta|$. We use $(0.13)$ for $\alpha$ and $\gamma$. Then,

$$
\left|x_{1}\right|^{\beta_{1}+\cdots+\beta_{d}}\left|D^{\alpha} f(x)\right|=\left|x^{\gamma} D^{\alpha} f(x)\right| \leq C_{\lambda, \mu} e^{\lambda \varphi^{*}\left(\frac{|\alpha|}{\lambda}\right)} e^{\left(\mu L^{\prime}+1\right) \varphi^{*}\left(\frac{|\gamma|}{\mu L^{\prime}+1}\right)},
$$

where $L^{\prime} \geq 1$ is the constant from (0.5). We take $j:=\beta_{1}+\cdots+\beta_{d}=|\beta|=$ $|\gamma| \in \mathbb{N}_{0}$, and from (0.8) and (0.5), we get

$$
\begin{aligned}
\left|D^{\alpha} f(x)\right| & \leq C_{\lambda, \mu} e^{\lambda \varphi^{*}\left(\frac{|\alpha|}{\lambda}\right)} \inf _{j \in \mathbb{N}_{0}}\left|x_{1}\right|^{-j} e^{\left(\mu L^{\prime}+1\right) \varphi^{*}\left(\frac{j}{\mu L^{\prime}+1}\right)} \\
& \leq C_{\lambda, \mu} e^{\lambda \varphi^{*}\left(\frac{|\alpha|}{\lambda}\right)} e^{-\left(\mu L^{\prime}+1\right) \omega\left(\left|x_{1}\right|\right)+\log \left|x_{1}\right|} \\
& \leq C_{\lambda, \mu}^{\prime} e^{\lambda \varphi^{*}\left(\frac{|\alpha|}{\lambda}\right)} e^{-\mu L^{\prime} \omega\left(\left|x_{1}\right|\right)} \leq C_{\lambda, \mu}^{\prime} e^{\mu L^{\prime}} e^{\lambda \varphi^{*}\left(\frac{|\alpha|}{\lambda}\right)} e^{-\mu \omega(x)},
\end{aligned}
$$


for some $C_{\lambda, \mu}^{\prime}>0$.

On the other hand we have that, by (0.7), for all $\mu>0, \beta \in \mathbb{N}_{0}^{d}$,

$$
\left|x^{\beta}\right|=\left|x_{1}\right|^{\beta_{1}} \cdots\left|x_{d}\right|^{\beta_{d}} \leq\langle x\rangle^{|\beta|} \leq e^{\mu \varphi^{*}\left(\frac{|\beta|}{\mu}\right)} e^{\mu \omega(\langle x\rangle)} .
$$

Therefore, by (0.6) and hypothesis, for all $\lambda, \mu>0$ there exists $C_{\lambda, \mu}>0$ so that

$$
\left|x^{\beta} D^{\alpha} f(x)\right| \leq\langle x\rangle^{|\beta|}\left|D^{\alpha} f(x)\right| \leq C_{\lambda, \mu} e^{\mu L} e^{\lambda \varphi^{*}\left(\frac{|\alpha|}{\lambda}\right)} e^{\mu \varphi^{*}\left(\frac{|\beta|}{\mu}\right)}
$$

for all $\alpha, \beta \in \mathbb{N}_{0}^{d}$ and $x \in \mathbb{R}^{d}$. This completes the proof.

Given $f \in \mathcal{S}_{\omega}\left(\mathbb{R}^{d}\right)$, for $\lambda>0$ we denote

$$
|f|_{\lambda}:=\sup _{\alpha \in \mathbb{N}_{0}^{d}} \sup _{x \in \mathbb{R}^{d}}\left|D^{\alpha} f(x)\right| e^{-\lambda \varphi^{*}\left(\frac{|\alpha|}{\lambda}\right)} e^{\lambda \omega(x)} .
$$

By the proof of Lemma $0.15,\left\{|\cdot|_{\lambda}\right\}_{\lambda>0}$ is a fundamental system of seminorms for $\mathcal{S}_{\omega}\left(\mathbb{R}^{d}\right)$. In [13, Theorem 4.8] (see also [15, Theorem 2.5]), one can find other equivalent system of seminorms of the space $\mathcal{S}_{\omega}\left(\mathbb{R}^{d}\right)$.

Proposition 0.16. The space $\mathcal{S}_{\omega}\left(\mathbb{R}^{d}\right) \otimes \mathcal{S}_{\omega}\left(\mathbb{R}^{d}\right)$ is dense in $\mathcal{S}_{\omega}\left(\mathbb{R}^{2 d}\right)$.

Proof. Let $f \in \mathcal{S}_{\omega}\left(\mathbb{R}^{2 d}\right)$. By the density of $\mathcal{D}_{(\omega)}\left(\mathbb{R}^{2 d}\right)$ in $\mathcal{S}_{\omega}\left(\mathbb{R}^{2 d}\right)$ (Remark 0.14), for all $\varepsilon, \lambda>0$ there exists $\psi \in \mathcal{D}_{(\omega)}\left(\mathbb{R}^{2 d}\right)$ such that

$$
\sup _{x \in \mathbb{R}^{2 d}}\left|D^{\alpha}(f(x)-\psi(x))\right| e^{-\lambda \varphi^{*}\left(\frac{|\alpha|}{\lambda}\right)} e^{\lambda \omega(x)}<\varepsilon / 2, \quad \alpha \in \mathbb{N}_{0}^{2 d} .
$$

Let $K_{1}$ and $K_{2}$ be compact sets such that $\operatorname{supp} \psi \subseteq K_{1} \times K_{2}$, and set

$$
M:=\sup _{x \in K_{1} \times K_{2}} e^{\omega(x)} .
$$

By [20, Theorem 8.1], for all $\varepsilon, \lambda>0$ there exists $\chi \in \mathcal{D}_{(\omega)}\left(K_{1}\right) \otimes \mathcal{D}_{(\omega)}\left(K_{2}\right)$ satisfying

$$
\sup _{x \in K_{1} \times K_{2}}\left|D^{\alpha}(\psi(x)-\chi(x))\right| e^{-\lambda \varphi^{*}\left(\frac{|\alpha|}{\lambda}\right)}<\frac{\varepsilon}{2 M^{\lambda}}, \quad \alpha \in \mathbb{N}_{0}^{2 d} .
$$


Therefore, for all $\alpha \in \mathbb{N}_{0}^{2 d}$,

$$
\begin{aligned}
\sup _{x \in \mathbb{R}^{2 d}} \mid & D^{\alpha}(f(x)-\chi(x)) \mid e^{-\lambda \varphi^{*}\left(\frac{|\alpha|}{\lambda}\right)} e^{\lambda \omega(x)} \\
\leq & \sup _{x \in \mathbb{R}^{2 d}}\left|D^{\alpha}(f(x)-\psi(x))\right| e^{-\lambda \varphi^{*}\left(\frac{|\alpha|}{\lambda}\right)} e^{\lambda \omega(x)}+ \\
& +\sup _{x \in K_{1} \times K_{2}} e^{\lambda \omega(x)} \cdot \sup _{x \in K_{1} \times K_{2}}\left|D^{\alpha}(\psi(x)-\chi(x))\right| e^{-\lambda \varphi^{*}\left(\frac{|\alpha|}{\lambda}\right)} \\
< & \frac{\varepsilon}{2}+M^{\lambda} \frac{\varepsilon}{2 M^{\lambda}}=\varepsilon .
\end{aligned}
$$

The dual space of $\mathcal{S}_{\omega}\left(\mathbb{R}^{d}\right)$ is denoted by $\mathcal{S}_{\omega}^{\prime}\left(\mathbb{R}^{d}\right)$, consisting of all the linear and continuous mappings $f: \mathcal{S}_{\omega}\left(\mathbb{R}^{d}\right) \rightarrow \mathbb{C}$. We say that an element of $\mathcal{S}_{\omega}^{\prime}\left(\mathbb{R}^{d}\right)$ is an $\omega$-temperate ultradistribution. The space $\mathcal{S}_{\omega}\left(\mathbb{R}^{d}\right)$ is dense in $\mathcal{S}_{\omega}^{\prime}\left(\mathbb{R}^{d}\right)$. Moreover, by Remark 0.14 we can identify $\mathcal{E}_{(\omega)}^{\prime}\left(\mathbb{R}^{d}\right)$ as a subspace of $\mathcal{S}_{\omega}^{\prime}\left(\mathbb{R}^{d}\right)$.

The Fourier transform of $T \in \mathcal{S}_{\omega}^{\prime}\left(\mathbb{R}^{d}\right)$ is defined by

$$
\langle\widehat{T}, \psi\rangle=\langle T, \widehat{\psi}\rangle, \quad \psi \in \mathcal{S}_{\omega}\left(\mathbb{R}^{d}\right)
$$

\subsection{Ultradifferential operators}

We introduce the ultradifferential operators of $(\omega)$-class (with constant coefficients). The notion of ultradifferential operator is used in structure theorems for ultradistributions (see Braun [19], Langenbruch [51]). Let $G \in \mathcal{H}\left(\mathbb{C}^{d}\right)$ be an entire function satisfying $\log |G|=O(\omega)$. For $\varphi \in \mathcal{E}_{(\omega)}\left(\mathbb{R}^{d}\right)$, the map $T_{G}: \mathcal{E}_{(\omega)}\left(\mathbb{R}^{d}\right) \rightarrow \mathbb{C}$ given by

$$
T_{G}(\varphi):=\sum_{\alpha \in \mathbb{N}_{0}^{d}} i^{|\alpha|} \frac{D^{\alpha} G(0)}{\alpha !} D^{\alpha} \varphi(0),
$$

defines an ultradistribution in $\mathcal{E}_{(\omega)}^{\prime}\left(\mathbb{R}^{d}\right)$, with support equal to $\{0\}$. The ultradifferential operator of $(\omega)$-class is defined as the convolution operator $G(D): \mathcal{D}_{(\omega)}^{\prime}\left(\mathbb{R}^{d}\right) \rightarrow \mathcal{D}_{(\omega)}^{\prime}\left(\mathbb{R}^{d}\right), \mu \mapsto T_{G} * \mu$.

In [19, Theorem 7] it is shown the existence of entire functions with prescribed exponential growth. The following theorem is taken from [51, Corollary 1.4]. We observe that condition $(\beta)$ (non-quasianalyticity) is not necessary. 
Theorem 0.17. Let $\omega:[0, \infty[\rightarrow[0, \infty[$ be a continuous and increasing function satisfying the conditions $(\alpha),(\gamma)$, and $(\delta)$ of Definition 0.3. Then there exist an even entire function $f \in \mathcal{H}(\mathbb{C})$ and $C_{1}, C_{2}, C_{3}>0$ such that

i) $\log |f(z)| \leq \omega(z)+C_{1}, \quad z \in \mathbb{C}$;

ii) $\log |f(z)| \geq C_{2} \omega(z), \quad$ for $z \in U:=\left\{z \in \mathbb{C}:|\operatorname{Im}(z)| \leq C_{3}(|\operatorname{Re}(z)|+1)\right\}$.

We prove the analogous result for several variables.

Theorem 0.18. Let $\omega$ satisfy the hypotheses of Theorem 0.17. Then there are an entire function $G \in \mathcal{H}\left(\mathbb{C}^{d}\right)$ and some constants $C_{1}, C_{2}, C_{3}, C_{4}>0$ such that

i') $\log |G(z)| \leq \omega(z)+C_{1}, \quad z \in \mathbb{C}^{d}$;

ii') $\log |G(z)| \geq C_{2} \omega(z)-C_{4}, \quad z \in \widetilde{U}:=\left\{z \in \mathbb{C}^{d}:|\operatorname{Im}(z)| \leq C_{3}(|\operatorname{Re}(z)|+1)\right\}$.

Proof. By Theorem 0.17, there exist an even entire function $f \in \mathcal{H}(\mathbb{C})$ and $C_{1}, C_{2}, C_{3}>0$ such that

$$
\begin{aligned}
& \log |f(z)| \leq \omega(z)+C_{1}, \quad z \in \mathbb{C} ; \\
& \log |f(z)| \geq C_{2} \omega(z), \quad z \in U:=\left\{z \in \mathbb{C}:|\operatorname{Im}(z)| \leq C_{3}(|\operatorname{Re}(z)|+1)\right\} .
\end{aligned}
$$

Since $f$ is even,

$$
f(z)=\sum_{n=0}^{\infty} a_{n} z^{2 n}, \quad a_{n} \in \mathbb{C}, n \in \mathbb{N}_{0} .
$$

It follows by $(0.17)$ that $\log |f(0)| \geq 0$, so $a_{0}$ is not zero. Now, fix $z=$ $\left(z_{1}, \ldots, z_{d}\right) \in \mathbb{C}^{d} \backslash\{0\}$ and put

$$
w=\sqrt{z_{1}^{2}+\cdots+z_{d}^{2}} \in \mathbb{C} .
$$

We define

$$
G(z)=\sum_{n=0}^{\infty} a_{n}\left(z_{1}^{2}+\cdots+z_{d}^{2}\right)^{n}=f(w) .
$$

The function $G$ is well defined and entire. We use (0.16) for $w$ and we obtain

$$
\log |G(z)|=\log |f(w)| \leq \omega(w)+C_{1} .
$$


This proves condition $i^{\prime}$ ), since

$$
\omega(w) \leq \omega\left(\sqrt{\left|z_{1}^{2}\right|+\cdots+\left|z_{d}^{2}\right|}\right)=\omega(z) .
$$

To prove $i i^{\prime}$ ), we first observe that for a small enough $0<\varepsilon<1,|\operatorname{Im}(z)|<$ $\varepsilon|\operatorname{Re}(z)|$ implies $w \in U$. Indeed, by the Cauchy-Schwarz inequality,

$$
\begin{aligned}
\left|\operatorname{Im}\left(z_{1}^{2}+\cdots+z_{d}^{2}\right)\right|=2\left|\sum_{j=1}^{d} \operatorname{Im}\left(z_{j}\right) \operatorname{Re}\left(z_{j}\right)\right| & <2 \sqrt{\sum_{j=1}^{d}\left|\operatorname{Im}\left(z_{j}\right)\right|^{2}} \sqrt{\sum_{j=1}^{d}\left|\operatorname{Re}\left(z_{j}\right)\right|^{2}} \\
& =2|\operatorname{Im}(z)||\operatorname{Re}(z)|<2 \varepsilon|\operatorname{Re}(z)|^{2}
\end{aligned}
$$

On the other hand,

$$
\begin{aligned}
\left|\operatorname{Re}\left(z_{1}^{2}+\cdots+z_{d}^{2}\right)\right| & =\sum_{j=1}^{d}\left(\left|\operatorname{Re}\left(z_{j}\right)\right|^{2}-\left|\operatorname{Im}\left(z_{j}\right)\right|^{2}\right) \\
& =|\operatorname{Re}(z)|^{2}-|\operatorname{Im}(z)|^{2}>|\operatorname{Re}(z)|^{2}\left(1-\varepsilon^{2}\right) .
\end{aligned}
$$

Therefore,

$$
\left|\frac{\operatorname{Im}\left(z_{1}^{2}+\cdots+z_{d}^{2}\right)}{\operatorname{Re}\left(z_{1}^{2}+\cdots+z_{d}^{2}\right)}\right| \leq \frac{2 \varepsilon|\operatorname{Re}(z)|^{2}}{|\operatorname{Re}(z)|^{2}\left(1-\varepsilon^{2}\right)}=\frac{2 \varepsilon}{1-\varepsilon^{2}}=\tan (\alpha),
$$

where $\alpha=\arctan \left(\frac{2 \varepsilon}{1-\varepsilon^{2}}\right)$. Hence, for $\varepsilon$ small enough,

$$
\left|\frac{\operatorname{Im}(w)}{\operatorname{Re}(w)}\right|=\left|\frac{\operatorname{Im}\left(\sqrt{z_{1}^{2}+\cdots+z_{d}^{2}}\right)}{\operatorname{Re}\left(\sqrt{z_{1}^{2}+\cdots+z_{d}^{2}}\right)}\right| \leq \tan \left(\frac{\alpha}{2}\right),
$$

where the right-hand side tends to 0 as $\varepsilon \rightarrow 0$. Therefore by (0.17) we have

$$
\begin{aligned}
\log |G(z)|=\log |f(w)| & \geq C_{2} \omega(w) \\
& =C_{2} \omega\left(\left|\sqrt{z_{1}^{2}+\cdots+z_{d}^{2}}\right|\right)=C_{2} \omega\left(\sqrt{\left|z_{1}^{2}+\cdots+z_{d}^{2}\right|}\right)
\end{aligned}
$$

for all $|\operatorname{Im}(z)|<\varepsilon|\operatorname{Re}(z)|$.

Let $q \in \mathbb{N}_{0}$ so that $2^{q} \geq \sqrt{d}$. Since $d\left|z_{1}^{2}+\cdots+z_{d}^{2}\right| \geq\left|z_{1}^{2}\right|+\cdots+\left|z_{d}^{2}\right|$, using $q$ times condition $(\alpha)$ of Definition 0.3 we have from (0.18) (as $L \geq 1$ )

$$
\begin{aligned}
\log |G(z)| & \geq C_{2} \omega\left(\frac{1}{\sqrt{d}} \sqrt{\left|z_{1}^{2}\right|+\cdots+\left|z_{d}^{2}\right|}\right) \\
& \geq \frac{C_{2}}{L^{q}} \omega\left(\frac{2^{q}}{\sqrt{d}}|z|\right)-C_{2} q \geq \frac{C_{2}}{L^{q}} \omega(z)-C_{2} q .
\end{aligned}
$$


Now, from the continuity of $G$ at 0 (notice that $|G(0)|=|f(0)| \geq 1$ ), there exists $0<\delta<1$ such that if $|z|<\delta$, then

$$
\log |G(z)| \geq C_{2}^{\prime} \omega(z)-C_{4}
$$

for some $C_{2}^{\prime}, C_{4}>0$. For the set $\widetilde{U}=\left\{z \in \mathbb{C}^{d}:|\operatorname{Im}(z)| \leq C_{3}(|\operatorname{Re}(z)|+1)\right\}$, we put $C_{3}:=\delta \varepsilon / 8>0$ and we show that if $z \in \widetilde{U}$, then

$$
\log |G(z)| \geq \widetilde{C_{2}} \omega(z)-\widetilde{C_{4}}
$$

for $\widetilde{C_{2}}:=\min \left\{C_{2} L^{-q}, C_{2}^{\prime}\right\}>0$ and $\widetilde{C_{4}}:=\max \left\{C_{2} q, C_{4}\right\}>0$. To this, we distinguish two cases: if $|\operatorname{Re}(z)| \leq \delta / 2$, then

$$
|\operatorname{Im}(z)| \leq C_{3}(|\operatorname{Re}(z)|+1) \leq \frac{\delta \varepsilon}{8}\left(\frac{\delta}{2}+1\right)<\frac{\delta}{4}\left(\frac{\delta}{2}+1\right)=\frac{\delta}{2}\left(\frac{\delta}{4}+\frac{1}{2}\right)<\frac{\delta}{2}
$$

Therefore $|z| \leq|\operatorname{Re}(z)|+|\operatorname{Im}(z)|<\delta$, and (0.20) is satisfied, and so is (0.21). On the other hand, if $|\operatorname{Re}(z)| \geq \delta / 2$, then

$$
\begin{aligned}
|\operatorname{Im}(z)| \leq C_{3}(|\operatorname{Re}(z)|+1) & =\frac{\delta \varepsilon}{8}|\operatorname{Re}(z)|+\frac{\delta \varepsilon}{8} \\
& <\frac{\varepsilon}{2}|\operatorname{Re}(z)|+\frac{\varepsilon}{2} \frac{\delta}{2} \leq \varepsilon|\operatorname{Re}(z)|,
\end{aligned}
$$

hence (0.19) holds, and also (0.21). The proof is complete.

In what follows, $G \in \mathcal{H}\left(\mathbb{C}^{d}\right)$ is the entire function of Theorem 0.18 .

Proposition 0.19. For the function $q(\xi):=G(\xi)^{-1}, \xi \in \mathbb{R}^{d}$, there exist $C, K, R>0$ such that

$$
\left|D^{\alpha} q(\xi)\right| \leq C \alpha ! R^{-|\alpha|} e^{-K \omega(\xi)}, \quad \alpha \in \mathbb{N}_{0}^{d}, \quad \xi \in \mathbb{R}^{d} .
$$

Proof. Let $\widetilde{U}$ and $C_{3}>0$ be the set and the constant appearing in condition $\left.i i^{\prime}\right)$ of Theorem 0.18. First we check that for the polyradius $r=(R, \ldots, R)$ with $0<\sqrt{d} R<C_{3}$, we have $\partial P(\xi, r) \subseteq \widetilde{U}$ for all $\xi=\left(\xi_{1}, \ldots, \xi_{d}\right) \in \mathbb{R}^{d}$. Indeed, if $z=\left(z_{1}, \ldots, z_{d}\right) \in \partial P(\xi, r)$, we have

$$
|\operatorname{Im}(z)| \leq \sqrt{d} \max _{1 \leq j \leq d}\left|\operatorname{Im}\left(z_{j}\right)\right|=\sqrt{d} \max _{1 \leq j \leq d}\left|\operatorname{Im}\left(z_{j}-\xi_{j}\right)\right| \leq \sqrt{d} \max _{1 \leq j \leq d}\left|z_{j}-\xi_{j}\right| .
$$

Then, by the choice of the polyradius $r$, we obtain

$$
|\operatorname{Im}(z)| \leq \sqrt{d} R<C_{3} \leq C_{3}(|\operatorname{Re}(z)|+1),
$$


as we wanted.

We use Proposition 0.11, and by Theorem 0.18 there exist $C_{2}, C_{4}>0$ such that

$$
\left|D^{\alpha} q(\xi)\right| \leq \frac{\alpha !}{r^{\alpha}} \sup _{z \in \partial P(\xi, r)}|q(z)| \leq e^{C_{4}} \frac{\alpha !}{r^{\alpha}} \sup _{z \in \partial P(\xi, r)} e^{-C_{2} \omega(z)}
$$

for all $\alpha \in \mathbb{N}_{0}^{d}, \xi \in \mathbb{R}^{d}$. We estimate the supremum on the right-hand side of $(0.22)$ : it is clear that

$$
-\omega(z) \leq-\frac{1}{d}\left(\omega\left(z_{1}\right)+\cdots+\omega\left(z_{d}\right)\right), \quad z=\left(z_{1}, \ldots, z_{d}\right) \in \partial P(\xi, r) .
$$

Since $\left|\xi_{j}\right|-\left|z_{j}\right| \leq\left|z_{j}-\xi_{j}\right|=R<C_{3} / \sqrt{d}$ for $j=1, \ldots, d$, we use formula $(0.1)$ to obtain

$$
-\omega\left(z_{j}\right) \leq-\omega\left(\left|\xi_{j}\right|-\frac{1}{\sqrt{d}} C_{3}\right) \leq-\frac{1}{L} \omega\left(\xi_{j}\right)+\omega\left(\frac{1}{\sqrt{d}} C_{3}\right)+1, \quad j=1, \ldots, d .
$$

By formula (0.5), we deduce, for that $L^{\prime} \geq 1$,

$$
\omega(\xi) \leq \omega\left(\sqrt{d}|\xi|_{\infty}\right) \leq L^{\prime} \omega\left(|\xi|_{\infty}\right)+L^{\prime} \leq L^{\prime}\left(\omega\left(\xi_{1}\right)+\cdots+\omega\left(\xi_{d}\right)\right)+L^{\prime}
$$

Therefore,

$$
-\left(\omega\left(\xi_{1}\right)+\cdots+\omega\left(\xi_{d}\right)\right) \leq-\frac{1}{L^{\prime}} \omega(\xi)+1 .
$$

Thus, we obtain, by $(0.23),(0.24)$ for all $j=1, \ldots, d$, and $(0.25)$,

$$
\begin{aligned}
-C_{2} \omega(z) & \leq-\frac{C_{2}}{d}\left(\omega\left(z_{1}\right)+\cdots+\omega\left(z_{d}\right)\right) \\
& \leq-\frac{C_{2}}{d L}\left(\omega\left(\xi_{1}\right)+\cdots+\omega\left(\xi_{d}\right)\right)+C_{2} \omega\left(\frac{1}{\sqrt{d}} C_{3}\right)+C_{2} \\
& \leq-\frac{C_{2}}{d L L^{\prime}} \omega(\xi)+\frac{C_{2}}{d L}+C_{2} \omega\left(\frac{1}{\sqrt{d}} C_{3}\right)+C_{2} .
\end{aligned}
$$

Since $r^{\alpha}=R^{|\alpha|}$, the result follows by $(0.22)$ for

$$
K=C_{2} /\left(d L L^{\prime}\right)>0, \quad C=e^{C_{4}} e^{C_{2} /(d L)+C_{2} \omega\left(C_{3} / \sqrt{d}\right)+C_{2}}>0 .
$$

The estimate in Proposition 0.19 can be adapted for any power of $G$ for the same constants. 
Corollary 0.20. For $n \in \mathbb{N}$, let $G^{n}$ denote the $n$-th power of $G$. Then, for $q^{n}(\xi):=G^{-n}(\xi), \xi \in \mathbb{R}^{d}$, it holds

$$
\left|D^{\alpha} q^{n}(\xi)\right| \leq C^{n} \alpha ! R^{-|\alpha|} e^{-n K \omega(\xi)},
$$

for the same constants $C, K, R>0$ as in Proposition 0.19 , for all $\alpha \in \mathbb{N}_{0}^{d}$, $\xi \in \mathbb{R}^{d}$.

Proof. Let $r$ be the same polyradius as in the proof of Proposition 0.19. Proceeding as in (0.22), we have

$$
\left|D^{\alpha} q^{n}(\xi)\right| \leq \frac{\alpha !}{r^{\alpha}} \sup _{z \in \partial P(\xi, r)}\left|q^{n}(z)\right| \leq e^{n C_{4}} \frac{\alpha !}{r^{\alpha}} \sup _{z \in \partial P(\xi, r)} e^{-n C_{2} \omega(z)},
$$

for all $\alpha \in \mathbb{N}_{0}^{d}, \xi \in \mathbb{R}^{d}$, where $C_{2}, C_{4}>0$ come from condition $\left.i i^{\prime}\right)$ of Theorem 0.18 . From (0.26) we deduce the result.

As $G(z)=\sum_{\alpha \in \mathbb{N}_{0}^{d}} a_{\alpha} z^{\alpha}$ for some sequence $\left\{a_{\alpha}\right\}_{\alpha} \subseteq \mathbb{C}$, for all $z \in \mathbb{C}^{d}$, for any $n \in \mathbb{N}$ we have $G^{n}(z)=\sum_{\alpha \in \mathbb{N}_{0}^{d}} b_{\alpha} z^{\alpha}$ for another sequence $\left\{b_{\alpha}\right\}_{\alpha} \subseteq \mathbb{C}$, for all $z \in \mathbb{C}^{d}$. To complete this section, we find suitable estimates for such sequences. We begin estimating the derivatives of $G$ at the origin.

Lemma 0.21. There exists $C>0$ depending on $G, \omega$ and $d$ such that

$$
\left|D^{\alpha} G(0)\right| \leq \alpha ! e^{C} e^{-C \varphi^{*}\left(\frac{|\alpha|}{C}\right)}, \quad \alpha \in \mathbb{N}_{0}^{d} .
$$

Proof. Let $R>0$ be arbitrary and set $r=(R, \ldots, R) \in \mathbb{R}^{d}$. Take $q \in \mathbb{N}_{0}$ so that $\sqrt{d} \leq 2^{q}$. By Proposition 0.11 and by condition $i^{\prime}$ ) of Theorem 0.18 , using $q$ times condition $(\alpha)$ of the weight function, there exist $C_{1}>0$ and $\bar{L}:=L^{q}+\cdots+L>0$ such that for all $\alpha \in \mathbb{N}_{0}^{d}$,

$$
\left|D^{\alpha} G(0)\right| \leq \frac{\alpha !}{r^{\alpha}} \sup _{z \in \partial P(0, r)}|G(z)| \leq \frac{\alpha !}{R^{|\alpha|}} e^{\omega(\sqrt{d} R)+C_{1}} \leq \frac{\alpha !}{R^{|\alpha|}} e^{\bar{L} \omega(R)+\bar{L}+C_{1}},
$$

for all $R>0$. Since

$$
\begin{aligned}
\inf _{R>0}\left\{R^{-|\alpha|} e^{\bar{L} \omega(R)}\right\} & =\left(\sup _{R>0}\left\{R^{|\alpha|} e^{-\bar{L} \omega(R)}\right\}\right)^{-1} \\
& \leq\left(\sup _{s>0}\left\{e^{s|\alpha|-\bar{L} \varphi(s)}\right\}\right)^{-1} \\
& =\left(\sup _{s>0}\left\{e^{\bar{L}(s(|\alpha| / \bar{L})-\varphi(s))}\right\}\right)^{-1}=e^{-\bar{L} \varphi^{*}\left(\frac{|\alpha|}{\bar{L}}\right)},
\end{aligned}
$$

we obtain the claim for $C:=\bar{L}+C_{1}>0$. 
We now give the analogous estimate for $G^{n}$ at the origin.

Corollary 0.22. Let $n \in \mathbb{N}$. For the same $C>0$ as in Lemma 0.21 it holds

$$
\left|D^{\alpha} G^{n}(0)\right| \leq \alpha ! e^{n C} e^{-n C \varphi^{*}\left(\frac{|\alpha|}{n C}\right)}, \quad \alpha \in \mathbb{N}_{0}^{d}
$$

Proof. Again, for arbitrary $R>0$ and $r=(R, \ldots, R) \in \mathbb{R}^{d}$, we obtain as in formula (0.27), that for all $\alpha \in \mathbb{N}_{0}^{d}$,

$$
\left|D^{\alpha} G^{n}(0)\right| \leq \frac{\alpha !}{r^{\alpha}} \sup _{z \in \partial P(0, r)}\left|G^{n}(z)\right| \leq \frac{\alpha !}{R^{|\alpha|}} e^{n \bar{L} \omega(R)+n \bar{L}+n C_{1}}
$$

for all $R>0$. As in (0.28),

$$
\begin{aligned}
\inf _{R>0}\left\{R^{-|\alpha|} e^{n \bar{L} \omega(R)}\right\} & \leq\left(\sup _{s>0}\left\{e^{s|\alpha|} e^{-n \bar{L} \varphi(s)}\right\}\right)^{-1} \\
& =\exp \left(n \bar{L} \sup _{s>0}\left\{s \frac{|\alpha|}{n \bar{L}}-\varphi(s)\right\}\right)^{-1}=\exp \left(n \bar{L} \varphi^{*}\left(\frac{|\alpha|}{n \bar{L}}\right)\right)^{-1} .
\end{aligned}
$$

Hence, the result follows.

Corollary 0.23. Let $n \in \mathbb{N}$. If $\left\{a_{\alpha}\right\}$ and $\left\{b_{\alpha}\right\}$ are the sequences such that

$$
G(z)=\sum_{\alpha \in \mathbb{N}_{0}^{d}} a_{\alpha} z^{\alpha}, \quad G^{n}(z)=\sum_{\alpha \in \mathbb{N}_{0}^{d}} b_{\alpha} z^{\alpha}, \quad z \in \mathbb{C}^{d},
$$

then for $C \geq 1$ as in Lemma 0.21,

$$
\begin{aligned}
& \left|a_{\alpha}\right| \leq e^{C} e^{-C \varphi^{*}\left(\frac{|\alpha|}{C}\right)}, \quad \alpha \in \mathbb{N}_{0}^{d} ; \\
& \left|b_{\alpha}\right| \leq e^{n C} e^{-n C \varphi^{*}\left(\frac{|\alpha|}{n C}\right)}, \quad \alpha \in \mathbb{N}_{0}^{d} .
\end{aligned}
$$

Proof. It is enough to use Lemma 0.21 and Corollary 0.22 and take into account that for arbitrary $\alpha \in \mathbb{N}_{0}^{d},\left|a_{\alpha}\right| \alpha ! \leq\left|D^{\alpha} G(0)\right|$, and $\left|b_{\alpha}\right| \alpha ! \leq\left|D^{\alpha} G^{n}(0)\right|$ for all $n \in \mathbb{N}$.

We denote $\check{T}(x):=T(-x)$. We define the convolution of $T \in \mathcal{E}_{(\omega)}^{\prime}\left(\mathbb{R}^{d}\right)$ and $\mu \in \mathcal{S}_{\omega}^{\prime}\left(\mathbb{R}^{d}\right)$ by (see [20, Definition 6.1])

$$
\langle T * \mu, \phi\rangle=\langle\mu, \check{T} * \phi\rangle, \quad \phi \in \mathcal{S}_{\omega}\left(\mathbb{R}^{d}\right) .
$$


Proposition 0.24. For an ultradifferential operator of $(\omega)$-class $G(D)$ we have that

$$
G(D): \mathcal{S}_{\omega}\left(\mathbb{R}^{d}\right) \rightarrow \mathcal{S}_{\omega}\left(\mathbb{R}^{d}\right), \quad G(D): \mathcal{S}_{\omega}^{\prime}\left(\mathbb{R}^{d}\right) \rightarrow \mathcal{S}_{\omega}^{\prime}\left(\mathbb{R}^{d}\right)
$$

are linear and continuous.

Proof. From [19], we deduce that for $f \in \mathcal{S}_{\omega}\left(\mathbb{R}^{d}\right)$, the operator $G(D)$ acts

$$
(G(D) f)(x):=\sum_{\alpha \in \mathbb{N}_{0}^{d}}(-i)^{|\alpha|} \frac{D^{\alpha} G(0)}{\alpha !} D^{\alpha} f(x) .
$$

Fix $f \in \mathcal{S}_{\omega}\left(\mathbb{R}^{d}\right)$ and $\lambda>0$. We have for all $\beta \in \mathbb{N}_{0}^{d}$,

$$
\left|D^{\beta}(G(D) f)(x)\right| \leq \sum_{\alpha \in \mathbb{N}_{0}^{d}} \frac{\left|D^{\alpha} G(0)\right|}{\alpha !}\left|D^{\alpha+\beta} f(x)\right| .
$$

By Lemma 0.21 there exists $C>0$ such that

$$
\left|D^{\alpha} G(0)\right| \leq \alpha ! e^{C} e^{-C \varphi^{*}\left(\frac{|\alpha|}{C}\right)}, \quad \alpha \in \mathbb{N}_{0}^{d} .
$$

Denoting $|f|_{\lambda}$ as in $(0.14)$, since $f \in \mathcal{S}_{\omega}\left(\mathbb{R}^{d}\right)$, we have for $\lambda^{\prime}:=\max \{\lambda, C L\}>0$

$$
\left|D^{\beta}(G(D) f)(x)\right| \leq \sum_{\alpha \in \mathbb{N}_{0}^{d}} e^{C} e^{-C \varphi^{*}\left(\frac{|\alpha|}{C}\right)}|f|_{2 \lambda^{\prime}} e^{2 \lambda^{\prime} \varphi^{*}\left(\frac{|\alpha+\beta|}{2 \lambda^{\prime}}\right)} e^{-2 \lambda^{\prime} \omega(x)}
$$

By (0.10) and (0.11), we have

$$
\begin{aligned}
\left|D^{\beta}(G(D) f)(x)\right| & \leq \sum_{\alpha \in \mathbb{N}_{0}^{d}} e^{C} e^{C L} e^{-|\alpha|} e^{-C L \varphi^{*}\left(\frac{|\alpha|}{C L}\right)}|f|_{2 \lambda^{\prime}} e^{C L \varphi^{*}\left(\frac{|\alpha|}{C L}\right)} e^{\lambda \varphi^{*}\left(\frac{|\beta|}{\lambda}\right)} e^{-\lambda \omega(x)} \\
& =e^{C+C L} e^{\lambda \varphi^{*}\left(\frac{|\beta|}{\lambda}\right)} e^{-\lambda \omega(x)}|f|_{2 \lambda^{\prime}} \sum_{\alpha \in \mathbb{N}_{0}^{d}} e^{-|\alpha|}
\end{aligned}
$$

Then, from Lemma 0.1 there exists $C^{\prime}>0$ such that

$$
\left|D^{\beta}(G(D) f)(x)\right| e^{-\lambda \varphi^{*}\left(\frac{|\beta|}{\lambda}\right)} e^{\lambda \omega(x)} \leq C^{\prime}|f|_{2 \lambda^{\prime}} .
$$

Hence $|G(D) f|_{\lambda} \leq C^{\prime}|f|_{2 \lambda^{\prime}}$ as we wanted.

This shows that $G(-D): \mathcal{S}_{\omega}\left(\mathbb{R}^{d}\right) \rightarrow \mathcal{S}_{\omega}\left(\mathbb{R}^{d}\right)$ is continuous and

$$
G(-D)^{t}: \mathcal{S}_{\omega}^{\prime}\left(\mathbb{R}^{d}\right) \rightarrow \mathcal{S}_{\omega}^{\prime}\left(\mathbb{R}^{d}\right)
$$


is also continuous. Therefore, for $\mu \in \mathcal{S}_{\omega}^{\prime}\left(\mathbb{R}^{d}\right), f \in \mathcal{S}_{\omega}\left(\mathbb{R}^{d}\right)$, we have (for the second equality, see [36, Proposición 1.2.4])

$$
\left\langle G(-D)^{t} \mu, f\right\rangle=\langle\mu, G(-D) f\rangle=\left\langle\mu, \check{T}_{G} * f\right\rangle=\left\langle T_{G} * \mu, f\right\rangle=\langle G(D) \mu, f\rangle .
$$

This shows the result.

\subsection{Time-frequency analysis}

Here we present some results regarding time-frequency analysis. Some methods of this theory will be used in Chapter 4. We denote the translation, the modulation, and the phase-shift operators by

$$
T_{x} f(y)=f(y-x) ; \quad M_{\xi} f(y)=e^{i y \cdot \xi} f(y) ; \quad \Pi(z) f(y)=e^{i y \cdot \xi} f(y-x),
$$

for all $x, y, \xi \in \mathbb{R}^{d}$ and $z=(x, \xi)$.

One of the fundamental tools of this theory is the short-time Fourier transform. We refer the reader to Gröchenig [38].

Definition 0.25. Let $\psi \in \mathcal{S}_{\omega}\left(\mathbb{R}^{d}\right) \backslash\{0\}$ be a window function. The short-time Fourier transform of $f \in \mathcal{S}_{\omega}^{\prime}\left(\mathbb{R}^{d}\right)$ is defined by

$$
V_{\psi} f(z):=\langle f, \Pi(z) \psi\rangle=\int_{\mathbb{R}^{d}} f(y) \overline{\psi(y-x)} e^{-i y \cdot \xi} d y, \quad z=(x, \xi) \in \mathbb{R}^{2 d} .
$$

We observe that the conjugate linear action of $\mathcal{S}_{\omega}^{\prime}\left(\mathbb{R}^{d}\right)$ on $\mathcal{S}_{\omega}\left(\mathbb{R}^{d}\right)$ is consistent with the scalar product in $L^{2}\left(\mathbb{R}^{d}\right),\langle\cdot, \cdot\rangle_{L^{2}\left(\mathbb{R}^{d}\right)}$. We can write the short-time Fourier transform in terms of the Fourier transform:

$$
V_{\psi} f(z)=\widehat{f \cdot T_{x} \bar{\psi}}(\xi), \quad z=(x, \xi) \in \mathbb{R}^{2 d},
$$

(see for example Gröchenig and Zimmermann [39]). The adjoint operator of the short-time Fourier transform is defined as follows: for $\psi \in L^{2}\left(\mathbb{R}^{d}\right)$, we write $A_{\psi}: L^{2}\left(\mathbb{R}^{2 d}\right) \rightarrow L^{2}\left(\mathbb{R}^{d}\right)$ for the operator given by

$$
A_{\psi} F=\int_{\mathbb{R}^{2 d}} F(z) \Pi(z) \psi d z .
$$

For all $F \in L^{2}\left(\mathbb{R}^{2 d}\right)$ and $g \in L^{2}\left(\mathbb{R}^{d}\right)$, we have

$$
\left\langle A_{\psi} F, g\right\rangle=\int_{\mathbb{R}^{2 d}} F(z)\langle\Pi(z) \psi, g\rangle d z=\int_{\mathbb{R}^{2 d}} F(z) \overline{V_{\psi} g(z)} d z=\left\langle F, V_{\psi} g\right\rangle=\left\langle V_{\psi}^{*} F, g\right\rangle .
$$


Hence, $A_{\psi}$ is the adjoint operator of $V_{\psi}: L^{2}\left(\mathbb{R}^{d}\right) \rightarrow L^{2}\left(\mathbb{R}^{2 d}\right)$. Thus, for $\psi \in \mathcal{S}_{\omega}\left(\mathbb{R}^{d}\right)$ and $F \in \mathcal{S}_{\omega}\left(\mathbb{R}^{2 d}\right)$, we define

$$
V_{\psi}^{*} F:=A_{\psi} F
$$

It is known that $V_{\psi}^{*}: \mathcal{S}_{\omega}\left(\mathbb{R}^{2 d}\right) \rightarrow \mathcal{S}_{\omega}\left(\mathbb{R}^{d}\right)$ is continuous (see for example [14, $(2.21)])$. Furthermore,

Lemma 0.26. If $\psi \in \mathcal{S}_{\omega}\left(\mathbb{R}^{d}\right) \backslash\{0\}$, then

$$
V_{\psi}: \mathcal{S}_{\omega}\left(\mathbb{R}^{d}\right) \rightarrow \mathcal{S}_{\omega}\left(\mathbb{R}^{2 d}\right), \quad V_{\psi}: \mathcal{S}_{\omega}^{\prime}\left(\mathbb{R}^{d}\right) \rightarrow \mathcal{S}_{\omega}^{\prime}\left(\mathbb{R}^{2 d}\right)
$$

are continuous. Moreover, if $u \in \mathcal{S}_{\omega}^{\prime}\left(\mathbb{R}^{d}\right)$, then there exist $c, \mu>0$ such that

$$
\left|V_{\psi} u(z)\right| \leq c e^{\mu \omega(z)}, \quad z \in \mathbb{R}^{2 d} .
$$

Proof. See [14, Propositions 2.9 and 4.7]. For the second inequality, we refer to [39, Theorem 2.4].

It follows from [39, Lemma 1.1] (see also [14, (2.25)]) that for all $f \in \mathcal{S}_{\omega}^{\prime}\left(\mathbb{R}^{d}\right)$, $g \in \mathcal{S}_{\omega}\left(\mathbb{R}^{d}\right)$, (see $[38,(3.17)]$ to understand the meaning of the integral)

$$
\left\langle V_{\psi}^{*} V_{\psi} f, g\right\rangle=\int_{\mathbb{R}^{2 d}} V_{\psi} f(z)\langle\Pi(z) \psi, g\rangle d z=(2 \pi)^{d}\|\psi\|_{L^{2}\left(\mathbb{R}^{d}\right)}^{2}\langle f, g\rangle .
$$

Therefore, we can show the following

Proposition 0.27. Let $u \in \mathcal{S}_{\omega}^{\prime}\left(\mathbb{R}^{d}\right)$ and $\psi, \phi \in \mathcal{S}_{\omega}\left(\mathbb{R}^{d}\right), \psi \neq 0$. Then,

$$
\left|V_{\phi} u(z)\right| \leq(2 \pi)^{-d}\|\psi\|_{L^{2}\left(\mathbb{R}^{d}\right)}^{-2}\left(\left|V_{\psi} u\right| *\left|V_{\phi} \psi\right|\right)(z), \quad z \in \mathbb{R}^{2 d} .
$$

We recall from [39, Theorem 2.7] a characterization of $\mathcal{S}_{\omega}\left(\mathbb{R}^{d}\right)$ in terms of the short-time Fourier transform.

Theorem 0.28. Let $\psi \in \mathcal{S}_{\omega}\left(\mathbb{R}^{d}\right) \backslash\{0\}$ be a window function, and let $f \in$ $\mathcal{S}_{\omega}^{\prime}\left(\mathbb{R}^{d}\right)$. The following assertions are equivalent:

(i) $f \in \mathcal{S}_{\omega}\left(\mathbb{R}^{d}\right)$.

(ii) For all $\lambda>0$, there exists $C_{\lambda}>0$ such that $\left|V_{\psi} f(z)\right| \leq C_{\lambda} e^{-\lambda \omega(z)}, z \in \mathbb{R}^{d}$.

(iii) $V_{\psi} f \in \mathcal{S}_{\omega}\left(\mathbb{R}^{2 d}\right)$. 
This provides also another equivalent system of seminorms for the space $\mathcal{S}_{\omega}\left(\mathbb{R}^{d}\right)$ (see e.g. [14, Proposition 2.10], cf. [15, Theorem 2.5(h)']): for $\psi \in \mathcal{S}_{\omega}\left(\mathbb{R}^{d}\right) \backslash\{0\}$,

$$
\left\|V_{\psi} f\right\|_{\omega, \lambda}:=\left\|V_{\psi} f(z) e^{\lambda \omega(z)}\right\|_{L^{\infty}\left(\mathbb{R}^{2 d}\right)}, \quad \lambda>0 .
$$

The following results are well known in the Schwartz class $\mathcal{S}\left(\mathbb{R}^{d}\right)$ (see for example [38, Chapter 3] or Grubb [40]). A similar proof of them remains valid for $\mathcal{S}_{\omega}\left(\mathbb{R}^{d}\right)$. We recall the inversion formula for the Fourier transform in $\mathcal{S}_{\omega}\left(\mathbb{R}^{d}\right)$ :

$$
f(x)=(2 \pi)^{-d} \int_{\mathbb{R}^{d}} e^{i y \cdot x} \widehat{f}(y) d y, \quad f \in \mathcal{S}_{\omega}\left(\mathbb{R}^{d}\right) .
$$

We will denote the inverse of the Fourier transform by

$$
\mathcal{F}^{-1}(f)(x)=(2 \pi)^{-d} \int e^{i y \cdot x} f(y) d y, \quad f \in \mathcal{S}_{\omega}\left(\mathbb{R}^{d}\right) .
$$

Lemma 0.29. If $T \in \mathcal{S}_{\omega}^{\prime}\left(\mathbb{R}^{d}\right)$ and $g \in \mathcal{S}_{\omega}\left(\mathbb{R}^{d}\right)$, then

$$
\widehat{g T}=(2 \pi)^{-d}(\widehat{g} * \widehat{T}), \quad \widehat{g * T}=\widehat{g} \cdot \widehat{T} .
$$

Lemma 0.30. If $f, g \in \mathcal{S}_{\omega}\left(\mathbb{R}^{d}\right) \backslash\{0\}$, then

$$
V_{g} f(x, \xi)=e^{-i x \cdot \xi} \overline{V_{f} g(-x,-\xi)}, \quad x, \xi \in \mathbb{R}^{d} .
$$

Lemma 0.31. If $\psi \in \mathcal{S}_{\omega}\left(\mathbb{R}^{d}\right) \backslash\{0\}$, then

$$
M_{y} \widehat{\psi}(\eta)=\widehat{T_{-y} \psi}(\eta), \quad \widehat{M_{y} \psi}(\eta)=T_{y} \widehat{\psi}(\eta), \quad y, \eta \in \mathbb{R}^{d} .
$$

Lemma 0.32. If $f \in \mathcal{S}_{\omega}^{\prime}\left(\mathbb{R}^{d}\right)$ and $\psi \in \mathcal{S}_{\omega}\left(\mathbb{R}^{d}\right) \backslash\{0\}$, then

$$
V_{\psi} f(x, \xi)=(2 \pi)^{-d}\left(\widehat{f} * M_{-x} \widehat{\bar{\psi}}\right)(\xi) \quad x, \xi \in \mathbb{R}^{d} .
$$

Proof. It is an immediate application of formula (0.30) and Lemmas 0.29 and 0.31 .

This result is taken from $[14,(4.31)]$.

Lemma 0.33. If $u \in \mathcal{S}_{\omega}^{\prime}\left(\mathbb{R}^{d}\right)$ and $\psi \in \mathcal{S}_{\omega}\left(\mathbb{R}^{d}\right) \backslash\{0\}$, then for all $\gamma \in \mathbb{N}_{0}^{d}$,

$$
V_{\psi}\left(D^{\gamma} u\right)(z)=\sum_{\beta \leq \gamma}\left(\begin{array}{l}
\gamma \\
\beta
\end{array}\right) \xi^{\gamma-\beta} V_{D^{\beta} \psi}(u)(z), \quad z=(x, \xi) \in \mathbb{R}^{2 d} .
$$


Proof. By definition, we have

$$
V_{\psi}\left(D^{\gamma} u\right)(z)=\left\langle D^{\gamma} u, \Pi(z) \psi\right\rangle=\left\langle u, D^{\gamma}(\Pi(z) \psi)\right\rangle .
$$

As

$$
D^{\gamma}(\Pi(z) \psi(y))=D_{y}^{\gamma}\left(e^{i y \cdot \xi} \psi(y-x)\right)=\sum_{\beta \leq \gamma}\left(\begin{array}{l}
\gamma \\
\beta
\end{array}\right) \xi^{\gamma-\beta} e^{i y \cdot \xi} D_{y}^{\beta} \psi(y-x),
$$

for all $y \in \mathbb{R}^{d}$, we then obtain

$$
V_{\psi}\left(D^{\gamma} u\right)(z)=\sum_{\beta \leq \gamma}\left(\begin{array}{l}
\gamma \\
\beta
\end{array}\right) \xi^{\gamma-\beta}\left\langle u, e^{i y \cdot \xi} D_{y}^{\beta} \psi(y-x)\right\rangle .
$$

Therefore, we get the result since $e^{i y \cdot \xi} D_{y}^{\beta} \psi(y-x)=\Pi(z) D^{\beta} \psi$ and using again the definition of short-time Fourier transform.

In Chapter 4, we will use the following

Definition 0.34. Let $f \in \mathcal{S}_{\omega}\left(\mathbb{R}^{2 d}\right)$. The Wigner transform of $f$ is

$$
\operatorname{Wig}(f)(x, \xi)=\int_{\mathbb{R}^{d}} e^{-i y \cdot \xi} f(x+y / 2, x-y / 2) d y, \quad x, \xi \in \mathbb{R}^{d} .
$$





\section{Chapter 1}

\section{Global pseudodifferential operators}

The local theory of pseudodifferential operators grew out of the study of singular integral operators, and developed after 1965 with the systematic studies of Kohn-Nirenberg [48], Hörmander [43], and others. Since then, several authors have studied pseudodifferential operators of finite or infinite order in Gevrey classes in the local sense; we mention, for instance, [41, 65]. We refer to Rodino [60] for an excellent introduction to this topic, and the references therein.

Gevrey classes are spaces of (non-quasianalytic) ultradifferentiable functions in between real analytic and $C^{\infty}$ functions. The study of several problems in general classes of ultradifferentiable functions has received much attention in the last 60 years. Here, we will work with ultradifferentiable functions as defined by Braun, Meise and Taylor [20], which define the classes in terms of the growth of the derivatives of the functions, or in terms of the growth of their Fourier transform (see, for example, Komatsu [49] and Björck [8], or [20], for two different points of view to define spaces of ultradifferentiable functions and ultradistributions; and Bonet, Meise, and Melikhov [18] for a comparison between the classes defined in [20,49]). 
In Fernández, Galbis, and Jornet [33], a full theory of pseudodifferential operators in the local sense is developed for ultradifferentiable classes of Beurling type as in [20], and it is proved that the corresponding operators are $\omega$-pseudolocal, and the product of two operators is given in terms of a suitable symbolic calculus. In [32, 34], the same authors construct a parametrix for such operators and study the action of the wave front set on them (see also Albanese, Jornet, and Oliaro [2] for a different point of view). On the other hand, very recently, Prangoski [58] studies pseudodifferential operators of global type and infinite order for ultradifferentiable classes of Beurling and Roumieu type in the sense of Komatsu, and later, in Cappiello, Pilipović, and Prangoski [25], a parametrix is constructed for such operators. See $[22,23,27,55,58]$ and the references therein for more examples of pseudodifferential operators in global classes (e.g., in Gelfand-Shilov classes).

The aim of this chapter is to study pseudodifferential operators of global type and infinite order in all the variables in classes of ultradifferentiable functions of Beurling type as introduced in [20]. Hence, the right setting is the class $\mathcal{S}_{\omega}\left(\mathbb{R}^{d}\right)$ as introduced by Björck [8]. We follow the lines of [58] and Shubin [64], but from the point of view of [33], in such a way that our proofs simplify the ones of [58]. Moreover, we clarify the role of some kind of entire functions [19, 51] (see Section 0.3) that become crucial throughout the text.

It is worth mentioning that in the case when the weight function satisfies (see [18, Corollary 16(3)]):

$$
\text { There exists } H>1: 2 \omega(t) \leq \omega(H t)+H, \quad t>0,
$$

the classes of ultradifferentiable functions defined by weights (as in [20]) and the ones defined by sequences (as in [49]) coincide. In this situation, the definition given by Prangoski for the Beurling case in [58, Definition 1] is expected to be the same as our Definition 1.3. But, if the weight sequence $\left(M_{p}\right)_{p}$ satisfies only condition $(M 2)$ of Komatsu, as it is assumed by [58], our classes of amplitudes could differ in general from the ones given by Prangoski (see [18, Example 17]). Hence, we are treating, even only in the Beurling setting, different cases.

We first introduce our global symbols and global amplitudes following [58, 64] and define the corresponding pseudodifferential operators. We give in Proposition 1.19 a characterization in terms of the kernel of an $\omega$-regularizing operator, which is a continuous linear operator $R: \mathcal{S}_{\omega}^{\prime}\left(\mathbb{R}^{d}\right) \rightarrow \mathcal{S}_{\omega}\left(\mathbb{R}^{d}\right)$. The $\omega$-regularizing operators are crucial to understand the symbolic calculus in the next chapter and, thus, to construct parametrices for pseudodifferential 
operators (see Chapter 3). We also see in Example 1.21 that many operators are pseudodifferential operators according to our definition.

The results of this chapter can be found in [6].

\subsection{Symbols and amplitudes}

We begin with the definitions of global symbol and global amplitude in our context of spaces of global (non-quasianalytic) ultradifferentiable functions of Beurling type, following Prangoski [58] and Shubin [64]. In the following, $m \in \mathbb{R}$ and $0<\rho \leq 1$.

Definition 1.1. A global symbol in $\mathrm{GS}_{\rho}^{m, \omega}$ is a function $p(x, \xi) \in C^{\infty}\left(\mathbb{R}^{2 d}\right)$ such that for all $\lambda>0$ there exists $C_{\lambda}>0$ with

$$
\left|D_{x}^{\alpha} D_{\xi}^{\beta} p(x, \xi)\right| \leq C_{\lambda}\langle(x, \xi)\rangle^{-\rho|\alpha+\beta|} e^{\lambda \rho \varphi^{*}\left(\frac{|\alpha+\beta|}{\lambda}\right)} e^{m \omega(x, \xi)},
$$

for all $\alpha, \beta \in \mathbb{N}_{0}^{d}, x, \xi \in \mathbb{R}^{d}$.

The symbols of Definition 1.1 are called of infinite order due to the term $e^{m \omega(x, \xi)}$. For the corresponding definition of finite order, we adapt [64, Definition 23.1] (see also [33]):

Definition 1.2. A global symbol of finite order in $\mathrm{S}_{\rho}^{m, \omega}$ is a function $p(x, \xi) \in$ $C^{\infty}\left(\mathbb{R}^{2 d}\right)$ such that for all $\lambda>0$ there exists $C_{\lambda}>0$ with

$$
\left|D_{x}^{\alpha} D_{\xi}^{\beta} p(x, \xi)\right| \leq C_{\lambda}\langle(x, \xi)\rangle^{-\rho|\alpha+\beta|} e^{\lambda \rho \varphi^{*}\left(\frac{|\alpha+\beta|}{\lambda}\right)}\langle(x, \xi)\rangle^{m},
$$

for all $\alpha, \beta \in \mathbb{N}_{0}^{d}, x, \xi \in \mathbb{R}^{d}$.

It follows from $(0.7)$ that $\mathrm{S}_{\rho}^{m, \omega} \subseteq \mathrm{GS}_{\rho}^{m, \omega}$.

Definition 1.3. A global amplitude in $\mathrm{GA}_{\rho}^{m, \omega}$ is a function $a(x, y, \xi) \in$ $C^{\infty}\left(\mathbb{R}^{3 d}\right)$ such that for all $\lambda>0$ there exists $C_{\lambda}>0$ with

$$
\left|D_{x}^{\alpha} D_{y}^{\gamma} D_{\xi}^{\beta} a(x, y, \xi)\right| \leq C_{\lambda}\left(\frac{\langle x-y\rangle}{\langle(x, y, \xi)\rangle}\right)^{\rho|\alpha+\gamma+\beta|} e^{\lambda \rho \varphi^{*}\left(\frac{|\alpha+\gamma+\beta|}{\lambda}\right)} e^{m \omega(x, y, \xi)},
$$

for all $\alpha, \gamma, \beta \in \mathbb{N}_{0}^{d}, x, y, \xi \in \mathbb{R}^{d}$.

Lemma 1.4. For every $x, y, \xi \in \mathbb{R}^{d}$ we have

$$
\langle x-y\rangle \leq \sqrt{2}\langle(x, y)\rangle \leq \sqrt{2}\langle(x, y, \xi)\rangle \leq \sqrt{6}\langle x-y\rangle\langle(x, \xi)\rangle .
$$


Proof. Since $(|x|-|y|)^{2} \geq 0$, we have $2|x||y| \leq|x|^{2}+|y|^{2}$. By the CauchySchwarz inequality we obtain

$$
\begin{aligned}
1+|x-y|^{2} & =1+|x|^{2}-2 x \cdot y+|y|^{2} \leq 1+|x|^{2}+2|x||y|+|y|^{2} \\
& \leq 1+2|x|^{2}+2|y|^{2}<2\left(1+|x|^{2}+|y|^{2}\right) .
\end{aligned}
$$

As $|y|^{2} \leq(|x-y|+|x|)^{2} \leq 2|x-y|^{2}+2|x|^{2}$, we get

$$
\begin{aligned}
1+|x|^{2}+|y|^{2}+|\xi|^{2} & \leq 1+|x|^{2}+|\xi|^{2}+2|x-y|^{2}+2|x|^{2} \\
& \leq 3\left(1+|x|^{2}+|x-y|^{2}+|\xi|^{2}\right) \\
& \leq 3\left(1+|x-y|^{2}\right)\left(1+|x|^{2}+|\xi|^{2}\right),
\end{aligned}
$$

and the result then follows.

It is immediate to check:

Example 1.5. Let $p(x, \xi)$ be a global symbol in $\mathrm{GS}_{\rho}^{m, \omega}$. Then $a_{1}(x, y, \xi):=$ $p(x, \xi)$ and $a_{2}(x, y, \xi):=p(y, \xi)$ are global amplitudes in $\mathrm{GA}_{\rho}^{\max \{0, m\}, \omega}$.

Proof. We need to estimate $\left|D_{x}^{\alpha} D_{y}^{\gamma} D_{\xi}^{\beta} a_{1}(x, y, \xi)\right|$ for all $\alpha, \gamma, \beta \in \mathbb{N}_{0}^{d}, x, y, \xi \in$ $\mathbb{R}^{d}$. We can assume $\gamma=0$ because $p(x, \xi)$ does not depend on the variable $y$. Since $p(x, \xi) \in \mathrm{GS}_{\rho}^{m, \omega}$, for all $\lambda>0$ there exists $C_{\lambda}=C_{\lambda L}>0$ such that

$$
\left|D_{x}^{\alpha} D_{\xi}^{\beta} a_{1}(x, y, \xi)\right| \leq C_{\lambda}\langle(x, \xi)\rangle^{-\rho|\alpha+\beta|} e^{\lambda L \rho \varphi^{*}\left(\frac{|\alpha+\beta|}{\lambda L}\right)} e^{m \omega(x, \xi)},
$$

for all $\alpha, \beta \in \mathbb{N}_{0}^{d}, x, y, \xi \in \mathbb{R}^{d}$. By Lemma 1.4 we obtain

$$
\langle(x, \xi)\rangle^{-\rho|\alpha+\beta|} \leq \sqrt{3}^{\rho|\alpha+\beta|}\left(\frac{\langle x-y\rangle}{\langle(x, y, \xi)\rangle}\right)^{\rho|\alpha+\beta|} .
$$

As $\sqrt{3} \leq e$, we use formula $(0.10)$ for $k=1$ and get

$$
\left(e^{\lambda L \varphi^{*}\left(\frac{|\alpha+\beta|}{\lambda L}\right)} e^{|\alpha+\beta|}\right)^{\rho} \leq e^{\lambda \rho \varphi^{*}\left(\frac{|\alpha+\beta|}{\lambda}\right)} e^{\lambda L \rho} .
$$

For $m \geq 0$, it follows that $m \omega(x, \xi) \leq m \omega(x, y, \xi)$. So, for all $\lambda>0$ there exists $C_{\lambda}^{\prime}=C_{\lambda} e^{\lambda L \rho}>0$ such that

$$
\left|D_{x}^{\alpha} D_{\xi}^{\beta} a_{1}(x, y, \xi)\right| \leq C_{\lambda}^{\prime}\left(\frac{\langle x-y\rangle}{\langle(x, y, \xi)\rangle}\right)^{\rho|\alpha+\beta|} e^{\lambda \rho \varphi^{*}\left(\frac{|\alpha+\beta|}{\lambda}\right)} e^{m \omega(x, y, \xi)},
$$

for every $\alpha, \beta \in \mathbb{N}_{0}^{d}, x, y, \xi \in \mathbb{R}^{d}$. This shows $a_{1} \in \mathrm{GA}_{\rho}^{m, \omega}$ for $m \geq 0$. An analogous proof works to see that $a_{2} \in \mathrm{GA}_{\rho}^{m, \omega}$. If $m<0$ the result is also clear. 
The following result can be seen as the reciprocal of Example 1.5.

Example 1.6. Let $a(x, y, \xi) \in \mathrm{GA}_{\rho}^{m, \omega}$ and $p(x, \xi):=\left.a(x, y, \xi)\right|_{y=x}$. Then $p \in \mathrm{GS}_{\rho}^{\max \{m, m L\}, \omega}$.

Proof. Let $\widetilde{p} \in \mathbb{N}_{0}$ so that $2 \leq e^{\rho \widetilde{p}}$. By the chain rule, from Definition 1.3 we have that for all $\lambda>0$ there exists $C_{\lambda}>0$ such that $\left(\right.$ as $\left.\sum\left(\begin{array}{l}\alpha \\ \widetilde{\alpha}\end{array}\right)=2^{|\alpha|}\right)$

$$
\begin{aligned}
\left|D_{x}^{\alpha} D_{\xi}^{\beta} a(x, y, \xi)\right|_{y=x} \mid & \leq \sum_{\widetilde{\alpha} \leq \alpha}\left(\begin{array}{c}
\alpha \\
\widetilde{\alpha}
\end{array}\right)\left|D_{x}^{\widetilde{\alpha}} D_{y}^{\alpha-\widetilde{\alpha}} D_{\xi}^{\beta} a(x, y, \xi)\right|_{y=x} \mid \\
& \leq C_{\lambda}\langle(x, x, \xi)\rangle^{-\rho|\alpha+\beta|} e^{\lambda L^{\widetilde{p}} \rho \varphi^{*}\left(\frac{|\alpha+\beta|}{\lambda L^{\tilde{p}}}\right)} 2^{|\alpha|} e^{m \omega(x, x, \xi)}
\end{aligned}
$$

for all $\alpha, \beta \in \mathbb{N}_{0}^{d}, x, \xi \in \mathbb{R}^{d}$. By (0.10), we have

$$
e^{\lambda L^{\widetilde{p}} \rho \varphi^{*}\left(\frac{|\alpha+\beta|}{\lambda L^{\widetilde{p}}}\right)} 2^{|\alpha|} \leq e^{\lambda \rho \varphi^{*}\left(\frac{|\alpha+\beta|}{\lambda}\right)} e^{\lambda \rho \sum_{j=1}^{\widetilde{p}} L^{j}} .
$$

From (0.4) it follows that

$$
\omega(x, \xi) \leq \omega(x, x, \xi) \leq L \omega(x, \xi)+L, \quad x, \xi \in \mathbb{R}^{d}
$$

The result holds since $\langle(x, \xi)\rangle \leq\langle(x, x, \xi)\rangle$.

Let $m_{1}, m_{2} \in \mathbb{R}$. It is clear that if $p_{1} \in \mathrm{GS}_{\rho}^{m_{1}, \omega}$ and $p_{2} \in \mathrm{GS}_{\rho}^{m_{2}, \omega}$ (respectively $a_{1} \in \mathrm{GA}_{\rho}^{m_{1}, \omega}$ and $a_{2} \in \mathrm{GA}_{\rho}^{m_{2}, \omega}$ ), then $p_{1} p_{2} \in \mathrm{GS}_{\rho}^{m_{1}+m_{2}, \omega}$ (respectively $a_{1} a_{2} \in$ $\left.\mathrm{GA}_{\rho}^{m_{1}+m_{2}, \omega}\right)$, and that if $m_{1} \leq m_{2}$, then $\mathrm{GS}_{\rho}^{m_{1}, \omega} \subseteq \mathrm{GS}_{\rho}^{m_{2}, \omega}$ and $\mathrm{GA}_{\rho}^{m_{1}, \omega} \subseteq$ $\mathrm{GA}_{\rho}^{m_{2}, \omega}$.

If $\omega_{2} \leq \omega_{1}$, as $\varphi_{\omega_{2}}^{*} \geq \varphi_{\omega_{1}}^{*}$ and $m \omega_{2} \geq m \omega_{1}$ for $m \leq 0$, it holds that GS $_{\rho}^{m, \omega_{1}} \subseteq$ $\mathrm{GS}_{\rho}^{m, \omega_{2}}$ for $m \leq 0$ (respectively, $\mathrm{GA}_{\rho}^{m, \omega_{1}} \subseteq \mathrm{GA}_{\rho}^{m, \omega_{2}}$ for $m \leq 0$ ). If $m>0$, we do not know if similar inclusions are true.

Moreover, if $0<\rho \leq \rho^{\prime} \leq 1$ we need to impose conditions on the weight functions $\omega_{1}$ and $\omega_{2}$ in the following way:

Example 1.7. Let $0<\rho_{2} \leq \rho_{1} \leq 1$. If $\omega_{1}$ and $\omega_{2}$ are weight functions such that

(1) $\omega_{2}\left(t^{\rho_{1} / \rho_{2}}\right)=O\left(\omega_{1}(t)\right)$, as $t \rightarrow \infty$, then there exists $C>0$ such that for $m \leq 0, \mathrm{GS}_{\rho_{1}}^{m, \omega_{1}} \subseteq \mathrm{GS}_{\rho_{2}}^{m C, \omega_{2}}$ and $\mathrm{GA}_{\rho_{1}}^{m, \omega_{1}} \subseteq \mathrm{GA}_{\rho_{2}}^{m C, \omega_{2}}$ 
(2) $\omega_{2}\left(t^{\rho_{1} / \rho_{2}}\right)=o\left(\omega_{1}(t)\right)$, as $t \rightarrow \infty$, then, for $m<0, \operatorname{GS}_{\rho_{1}}^{m, \omega_{1}} \subseteq \bigcap_{k \in \mathbb{R}} \operatorname{GS}_{\rho_{2}}^{k, \omega_{2}}$ and $\mathrm{GA}_{\rho_{1}}^{m, \omega_{1}} \subseteq \bigcap_{k \in \mathbb{R}} \mathrm{GA}_{\rho_{2}}^{k, \omega_{2}}$.

Proof. (1) As $\rho_{2} \leq \rho_{1}$, by assumption there exists $C>0$ such that $\omega_{2}(t) \leq$ $\omega_{2}\left(t^{\rho_{1} / \rho_{2}}\right) \leq C \omega_{1}(t)+C$ for all $t \geq 0$. Thus

$$
m \omega_{1}(t) \leq m C^{-1} \omega_{2}(t)-m, \quad t \geq 0,
$$

for $m \leq 0$. Moreover, we use Lemma $0.10(2)$ to get that there exists $C^{\prime}>0$ so that for all $\lambda>0, j \in \mathbb{N}_{0}$,

$$
\lambda C^{\prime} \varphi_{\omega_{1}}^{*}\left(\frac{j}{\lambda C^{\prime}}\right) \leq \lambda C^{\prime}+\lambda \frac{\rho_{2}}{\rho_{1}} \varphi_{\omega_{2}}^{*}\left(\frac{j}{\lambda}\right) .
$$

Since $\langle(x, \xi)\rangle^{-\rho_{1}|\alpha+\beta|} \leq\langle(x, \xi)\rangle^{-\rho_{2}|\alpha+\beta|}$ for all $x, \xi \in \mathbb{R}^{d}$, the result follows for symbols. Now, let $a \in \mathrm{GA}_{\rho_{1}}^{m, \omega_{1}}$. By Definition 1.3, for all $\lambda>0$ there exists $C_{\lambda}=C_{\lambda C^{\prime} L}>0$ such that

$$
\left|D_{x}^{\alpha} D_{y}^{\gamma} D_{\xi}^{\beta} a(x, y, \xi)\right| \leq C_{\lambda}\left(\frac{\langle x-y\rangle}{\langle(x, y, \xi)\rangle}\right)^{\rho_{1}|\alpha+\gamma+\beta|} e^{\lambda C^{\prime} L \rho_{1} \varphi_{\omega_{1}}^{*}\left(\frac{|\alpha+\gamma+\beta|}{\lambda C^{\prime} L}\right)} e^{m \omega_{1}(x, y, \xi)},
$$

for all $\alpha, \gamma, \beta \in \mathbb{N}_{0}^{d}, x, y, \xi \in \mathbb{R}^{d}$. By Lemma 1.4, as $0 \leq \rho_{1}-\rho_{2}<\rho_{1}$,

$$
\left(\frac{\langle x-y\rangle}{\langle(x, y, \xi)\rangle}\right)^{\rho_{1}|\alpha+\gamma+\beta|} \leq\left(\frac{\langle x-y\rangle}{\langle(x, y, \xi)\rangle}\right)^{\rho_{2}|\alpha+\gamma+\beta|} \sqrt{2}^{\rho_{1}|\alpha+\gamma+\beta|} .
$$

Since $\sqrt{2} \leq e$, from formula (0.10) for $k=1$ and (1.2) we obtain

$$
\begin{aligned}
\left(e^{\lambda C^{\prime} L \varphi_{\omega_{1}}^{*}\left(\frac{|\alpha+\gamma+\beta|}{\lambda C^{\prime} L}\right)} e^{|\alpha+\gamma+\beta|}\right)^{\rho_{1}} & \leq e^{\lambda C^{\prime} \rho_{1} \varphi_{\omega_{1}}^{*}\left(\frac{|\alpha+\gamma+\beta|}{\lambda C^{\prime}}\right)} e^{\lambda C^{\prime} L \rho_{1}} \\
& \leq e^{\lambda C^{\prime} L \rho_{1}} e^{\lambda C^{\prime} \rho_{1}} e^{\lambda \rho_{2} \varphi_{\omega_{2}}^{*}\left(\frac{|\alpha+\gamma+\beta|}{\lambda}\right)} .
\end{aligned}
$$

By (1.1) we obtain $a \in \mathrm{GA}_{\rho_{2}}^{m C^{-1}, \omega_{2}}$.

(2) By the hypothesis, similarly as in (1.1) and (1.2), for $-m>0$ and given $k>0$ there exists $C_{k, m}>0$ such that $k \omega_{2}(t) \leq-m \omega_{1}(t)+C_{k, m}$. Hence

$$
m \omega_{1}(t) \leq-k \omega_{2}(t)+C_{k, m}, \quad t \geq 0 .
$$

Moreover, by Lemma $0.10(1)$, for all $\lambda>0$ there exists $C_{\lambda}>0$ such that

$$
\lambda \varphi_{\omega_{1}}^{*}\left(\frac{j}{\lambda}\right) \leq C_{\lambda}+\lambda \frac{\rho_{2}}{\rho_{1}} \varphi_{\omega_{2}}^{*}\left(\frac{j}{\lambda}\right), \quad j \in \mathbb{N}_{0} .
$$

As before, the result for symbols follows due to the arbitrariness of $k>0$. For amplitudes we replace (1.2) by (1.3) and proceed in the same way. 
Now, take a weight function $\sigma$ such that $\omega\left(t^{1 / \rho}\right)=o(\sigma(t)), t \rightarrow \infty$. We show that if $f \in \mathcal{D}_{\{\sigma\}}\left(\mathbb{R}^{2 d}\right)$, then $f \in \bigcap_{m \in \mathbb{R}} \mathrm{GS}_{\rho}^{m, \omega}$. Indeed, there exist $n, C>0$ such that

$$
\left|D_{x}^{\alpha} D_{\xi}^{\beta} f(x, \xi)\right| \leq C e^{\frac{1}{n} \varphi_{\sigma}^{*}(n|\alpha+\beta|)}, \quad \alpha, \beta \in \mathbb{N}_{0}^{d}, x, \xi \in \mathbb{R}^{d} .
$$

We take $R \in \mathbb{N}$ such that $f(x, \xi)=0$ for $\langle(x, \xi)\rangle \geq e^{R}$. By Lemma 0.10, we get that for all $\lambda>0$ there exists $C_{\lambda}=C e^{C_{\lambda L},_{n}}>0$ such that

$$
\left|D_{x}^{\alpha} D_{\xi}^{\beta} f(x, \xi)\right| \leq C_{\lambda} e^{\lambda L^{R} \rho \varphi_{\omega}^{*}\left(\frac{|\alpha+\beta|}{\lambda L^{R}}\right)}, \quad \alpha, \beta \in \mathbb{N}_{0}^{d}, x, \xi \in \mathbb{R}^{d} .
$$

For $\langle(x, \xi)\rangle \leq e^{R}$, we have by $(0.10)$

$$
\begin{aligned}
e^{\lambda L^{R} \rho \varphi_{\omega}^{*}\left(\frac{|\alpha+\beta|}{\lambda L^{R}}\right)} & \leq\langle(x, \xi)\rangle^{-\rho|\alpha+\beta|}\left(e^{R|\alpha+\beta|} e^{\lambda L^{R} \varphi_{\omega}^{*}\left(\frac{|\alpha+\beta|}{\lambda L^{R}}\right)}\right)^{\rho}\left(e^{-\lambda \omega(x, \xi)} e^{\lambda \omega\left(e^{R}\right)}\right) \\
& \leq\langle(x, \xi)\rangle^{-\rho|\alpha+\beta|} e^{\lambda \rho \varphi_{\omega}^{*}\left(\frac{|\alpha+\beta|}{\lambda}\right)} e^{\lambda \rho \sum_{j=1}^{R} L^{j}} e^{-\lambda \omega(x, \xi)} e^{\lambda \omega\left(e^{R}\right)} .
\end{aligned}
$$

This shows the result. The same argument works to show that if $f \in \mathcal{D}_{(\omega)}\left(\mathbb{R}^{2 d}\right)$, then $f \in \bigcap_{m \in \mathbb{R}} \mathrm{GS}_{1}^{m, \omega}$. We will discuss similar inclusions in Example 1.21(b).

\subsection{Continuity of the operator}

We define pseudodifferential operators for global amplitudes as in Definition 1.3 using oscillatory integrals. Let $\chi \in \mathcal{S}_{\omega}\left(\mathbb{R}^{2 d}\right)$ with $\chi(0)=1$. We consider, for $n \in \mathbb{N}$, the double integral for arbitrary $f \in \mathcal{S}_{\omega}\left(\mathbb{R}^{d}\right), x \in \mathbb{R}^{d}$, given by

$$
A_{\frac{1}{n}, \chi}(f)(x):=\iint_{\mathbb{R}^{d} \times \mathbb{R}^{d}} e^{i(x-y) \cdot \xi} a(x, y, \xi) \chi\left(\frac{1}{n}(x, \xi)\right) f(y) d y d \xi .
$$

We prove that $\left\{A_{\frac{1}{n}, \chi}(f)\right\}$ converges for every $f \in \mathcal{S}_{\omega}\left(\mathbb{R}^{d}\right)$ when $n \rightarrow \infty$. This limit will define a linear and continuous operator $A: \mathcal{S}_{\omega}\left(\mathbb{R}^{d}\right) \rightarrow \mathcal{S}_{\omega}\left(\mathbb{R}^{d}\right)$ given by the iterated integral

$$
A(f)(x):=\int_{\mathbb{R}^{d}}\left(\int_{\mathbb{R}^{d}} e^{i(x-y) \cdot \xi} a(x, y, \xi) f(y) d y\right) d \xi, \quad f \in \mathcal{S}_{\omega}\left(\mathbb{R}^{d}\right) .
$$

Hence, the operator in (1.5) is independent of the choice of the test function $\chi \in \mathcal{S}_{\omega}\left(\mathbb{R}^{2 d}\right)$ in $(1.4)$.

We use a suitable integration by parts with an ultradifferential operator of $(\omega)$-class as in Section 0.3, which will be also useful for next chapters. 
Lemma 1.8. Let $G$ be the entire function of Theorem 0.18. For every $n \in \mathbb{N}$,

$$
\begin{aligned}
e^{i(x-y) \cdot \xi} & =\frac{1}{G^{n}(\xi)} G^{n}\left(-D_{y}\right) e^{i(x-y) \cdot \xi} \\
& =\frac{1}{G^{n}(y-x)} G^{n}\left(-D_{\xi}\right) e^{i(x-y) \cdot \xi} \\
& =\frac{1}{G^{n}(y-x)} G^{n}\left(-D_{\xi}\right)\left(\frac{1}{G^{n}(\xi)} G^{n}\left(-D_{y}\right) e^{i(x-y) \cdot \xi}\right) .
\end{aligned}
$$

Proof. Since $G^{n}(D)=\sum_{\alpha \in \mathbb{N}_{0}^{d}} b_{\alpha} D^{\alpha}$, for some sequence $\left\{b_{\alpha}\right\} \subseteq \mathbb{C}$, we have (notice that $e^{i(x-y) \cdot \xi} \in \mathcal{E}_{(\omega)}\left(\mathbb{R}_{y}^{d}\right)$ for all $x, \xi \in \mathbb{R}^{d}$ )

$$
\begin{aligned}
G^{n}\left(-D_{y}\right)\left(e^{i(x-y) \cdot \xi}\right) & =\sum_{\alpha \in \mathbb{N}_{0}^{d}} b_{\alpha}\left(-D_{y}\right)^{\alpha}\left(e^{i(x-y) \cdot \xi}\right) \\
& =\sum_{\alpha \in \mathbb{N}_{0}^{d}} b_{\alpha}(-1)^{|\alpha|}(-i)^{|\alpha|}(-i \xi)^{\alpha} e^{i(x-y) \cdot \xi}=e^{i(x-y) \cdot \xi} G^{n}(\xi) .
\end{aligned}
$$

This shows (1.6). For (1.7), we can proceed similarly. A combination of (1.6) and (1.7) yields (1.8).

Proposition 1.9. Let $\chi \in \mathcal{S}_{\omega}\left(\mathbb{R}^{2 d}\right)$. For every function $f \in \mathcal{S}_{\omega}\left(\mathbb{R}^{d}\right)$, the sequence $\left\{A_{\frac{1}{n}, \chi}(f)\right\}_{n \in \mathbb{N}}$ as in (1.4) is a Cauchy sequence in $\mathcal{S}_{\omega}\left(\mathbb{R}^{d}\right)$.

Proof. According to the notation (0.14), for any $f \in \mathcal{S}_{\omega}\left(\mathbb{R}^{d}\right)$ and $\lambda>0$, we need to show that

$$
\left|\left(A_{1 / k, \chi}-A_{1 / l, \chi}\right)(f)\right|_{\lambda} \rightarrow 0
$$

as $l, k$ tend to infinity.

To this aim, we differentiate $\left(A_{1 / k, \chi}-A_{1 / l, \chi}\right)(f)$ under the integral sign, using Leibniz rule, as follows:

$$
\begin{aligned}
D_{x}^{\alpha}\left(\iint_{\mathbb{R}^{d} \times \mathbb{R}^{d}} e^{i(x-y) \cdot \xi} a(x, y, \xi)\left(\chi\left(\frac{1}{k}(x, \xi)\right)-\chi\left(\frac{1}{l}(x, \xi)\right)\right) f(y) d y d \xi\right) \\
=\sum_{\alpha_{1}+\alpha_{2}+\alpha_{3}=\alpha} \frac{\alpha !}{\alpha_{1} ! \alpha_{2} ! \alpha_{3} !} \iint_{\mathbb{R}^{2 d}} e^{i(x-y) \cdot \xi} \xi^{\alpha_{1}} D_{x}^{\alpha_{2}} a(x, y, \xi) \times \\
\quad \times D_{x}^{\alpha_{3}}\left(\chi\left(\frac{1}{k}(x, \xi)\right)-\chi\left(\frac{1}{l}(x, \xi)\right)\right) f(y) d y d \xi
\end{aligned}
$$

for all $\alpha \in \mathbb{N}_{0}^{d}$ and $x \in \mathbb{R}^{d}$. Taking into account the sequences in Corollary 0.23, we make an integration by parts via formula (1.8) for a suitable power $n \in \mathbb{N}$ 
(that we determine later) to obtain the following expression for the integrand in $(1.10)$ :

$$
\begin{aligned}
e^{i(x-y) \cdot \xi} \frac{1}{G^{n}(\xi)} G^{n}\left(D_{y}\right)\left(\frac { 1 } { G ^ { n } ( y - x ) } G ^ { n } ( D _ { \xi } ) \left(\xi^{\alpha_{1}} D_{x}^{\alpha_{2}} a(x, y, \xi) \times\right.\right. \\
\left.\left.\quad \times D_{x}^{\alpha_{3}}\left(\chi\left(\frac{1}{k}(x, \xi)\right)-\chi\left(\frac{1}{l}(x, \xi)\right)\right) f(y)\right)\right) \\
=e^{i(x-y) \cdot \xi} \frac{1}{G^{n}(\xi)} G^{n}\left(D_{y}\right)\left(\frac{1}{G^{n}(y-x)} \sum_{\eta \in \mathbb{N}_{0}^{d}} b_{\eta} \sum_{\eta_{1}+\eta_{2}+\eta_{3}=\eta, \eta_{1} \leq \alpha_{1}} \frac{\eta !}{\eta_{1} ! \eta_{2} ! \eta_{3} !} \times\right. \\
\quad \times \frac{\alpha_{1} !}{\left(\alpha_{1}-\eta_{1}\right) !}(-i)^{\left|\eta_{1}\right|} \xi^{\alpha_{1}-\eta_{1}} D_{x}^{\alpha_{2}} D_{\xi}^{\eta_{2}} a(x, y, \xi) \times \\
\left.\quad \times D_{x}^{\alpha_{3}} D_{\xi}^{\eta_{3}}\left(\chi\left(\frac{1}{k}(x, \xi)\right)-\chi\left(\frac{1}{l}(x, \xi)\right)\right) f(y)\right) \\
e^{i(x-y) \cdot \xi} \frac{1}{G^{n}(\xi)} \sum_{\epsilon, \eta \in \mathbb{N}_{0}^{d}} b_{\epsilon} b_{\eta} \quad \sum_{\eta_{1}+\eta_{2}+\eta_{3}=\eta, \epsilon_{1} \leq \alpha_{1}} \frac{\epsilon !}{\epsilon_{1} ! \epsilon_{2} ! \epsilon_{3} !} \frac{\eta !}{\eta_{1} ! \eta_{2} ! \eta_{3} !} \frac{\alpha_{1} !}{\left(\alpha_{1}-\eta_{1}\right) !} \times \\
\quad \times(-i)^{\left|\eta_{1}\right|} \xi^{\alpha_{1}-\eta_{1}} D_{y}^{\epsilon_{1}} \frac{1}{G^{n}(y-x)} D_{x}^{\alpha_{2}} D_{y}^{\epsilon_{2}} D_{\xi}^{\eta_{2}} a(x, y, \xi) \times \\
\quad \times D_{x}^{\alpha_{3}} D_{\xi}^{\eta_{3}}\left(\chi\left(\frac{1}{k}(x, \xi)\right)-\chi\left(\frac{1}{l}(x, \xi)\right)\right) D_{y}^{\epsilon_{3}} f(y) .
\end{aligned}
$$

Hence, (1.10) is equal to

$$
\begin{aligned}
& \sum_{\epsilon, \eta \in \mathbb{N}_{0}^{d}} b_{\epsilon} b_{\eta} \sum_{\begin{array}{c}
\alpha_{1}+\alpha_{2}+\alpha_{3}=\alpha \\
\epsilon_{1}+\epsilon_{2}+\epsilon_{3}=\epsilon \\
\eta_{1}+\eta_{2}+\eta_{3}=\eta, \eta_{1} \leq \alpha_{1}
\end{array}} \frac{\alpha !}{\alpha_{1} ! \alpha_{2} ! \alpha_{3} !} \frac{\epsilon !}{\epsilon_{1} ! \epsilon_{2} ! \epsilon_{3} !} \frac{\eta !}{\eta_{1} ! \eta_{2} ! \eta_{3} !} \frac{\alpha_{1} !}{\left(\alpha_{1}-\eta_{1}\right) !} \times \\
& \quad \times \iint_{\mathbb{R}^{2 d}} e^{i(x-y) \cdot \xi} \frac{(-i)^{\left|\eta_{1}\right|}}{G^{n}(\xi)} \xi^{\alpha_{1}-\eta_{1}} D_{y}^{\epsilon_{1}} \frac{1}{G^{n}(y-x)} D_{x}^{\alpha_{2}} D_{y}^{\epsilon_{2}} D_{\xi}^{\eta_{2}} a(x, y, \xi) \times \\
& \times D_{x}^{\alpha_{3}} D_{\xi}^{\eta_{3}}\left(\chi\left(\frac{1}{k}(x, \xi)\right)-\chi\left(\frac{1}{l}(x, \xi)\right)\right) D_{y}^{\epsilon_{3}} f(y) d y d \xi .
\end{aligned}
$$

Now we fix $\lambda>0$ and we take $s \geq \lambda$ to be determined. Since $f \in \mathcal{S}_{\omega}\left(\mathbb{R}^{d}\right)$, for that $s>0$ there exists $C_{s}=C_{s L^{3}}>0$ such that

$$
\left|D^{\epsilon_{3}} f(y)\right| \leq C_{s} e^{s L^{3} \varphi^{*}\left(\frac{\left|\epsilon_{3}\right|}{s L^{3}}\right)} e^{-s L^{3} \omega(y)} .
$$


Moreover, by Definition 1.3 there exists $C_{s}^{\prime}=C_{4 s L^{4}}^{\prime}>0$ so that we obtain, by Lemma 1.4 and (0.10),

$$
\begin{aligned}
& \left|D_{x}^{\alpha_{2}} D_{y}^{\epsilon_{2}} D_{\xi}^{\eta_{2}} a(x, y, \xi)\right| \leq C_{s}^{\prime}\left(\frac{\langle x-y\rangle}{\langle(x, y, \xi)\rangle}\right)^{\rho\left|\alpha_{2}+\epsilon_{2}+\eta_{2}\right|} e^{4 s L^{4} \rho \varphi^{*}\left(\frac{\left|\alpha_{2}+\epsilon_{2}+\eta_{2}\right|}{4 s L^{4}}\right)} e^{m \omega(x, y, \xi)} \\
& \leq C_{s}^{\prime} \sqrt{2}^{\left|\alpha_{2}+\epsilon_{2}+\eta_{2}\right|} e^{4 s L^{4} \varphi^{*}\left(\frac{\left|\alpha_{2}+\epsilon_{2}+\eta_{2}\right|}{4 s L^{4}}\right)} e^{m \omega(x, y, \xi)} \\
& \leq C_{s}^{\prime} e^{4 s L^{4}} e^{4 s L^{3} \varphi^{*}\left(\frac{\left|\alpha_{2}+\epsilon_{2}+\eta_{2}\right|}{4 s L^{3}}\right)} e^{m \omega(x, y, \xi)} .
\end{aligned}
$$

By formula (0.7) we have if $|\xi| \geq 1$ (if $|\xi| \leq 1$, then $|\xi|^{\left|\alpha_{1}-\eta_{1}\right|} \leq 1$ )

$$
|\xi|^{\left|\alpha_{1}-\eta_{1}\right|} \leq e^{\lambda L^{3} \varphi^{*}\left(\frac{\left|\alpha_{1}-\eta_{1}\right|}{\lambda L^{3}}\right)} e^{\lambda L^{3} \omega(\xi)} .
$$

On the other hand, by Corollary 0.23 there exists $C_{1}>0$ depending only on $G$ so that

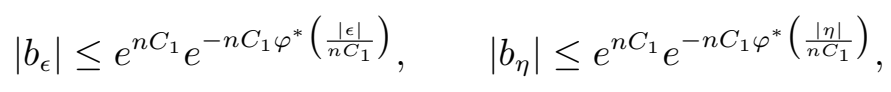

and from Corollary 0.20 there exist $C_{2}, C_{3}, C_{4}>0$ that depend on $G, \omega$, and the dimension $d$ such that, by formula $(0.12)$

$$
\begin{aligned}
\left|\frac{1}{G^{n}(\xi)}\right| & \leq C_{3}^{n} e^{-n C_{2} \omega(\xi)} ; \\
\left|D_{y}^{\epsilon_{1}} \frac{1}{G^{n}(y-x)}\right| & \leq C_{3}^{n} \epsilon_{1} ! C_{4}^{-\left|\epsilon_{1}\right|} e^{-n C_{2} \omega(y-x)} \leq C_{3}^{n} C_{s}^{\prime \prime} e^{s L^{3} \varphi^{*}\left(\frac{\left|\epsilon_{1}\right|}{s L^{3}}\right)} e^{-n C_{2} \omega(y-x),},
\end{aligned}
$$

for some constant $C_{s}^{\prime \prime}=C_{s L^{3}}^{\prime \prime}>0$. The same formula (0.12) guarantees the existence of $C_{s}^{\prime \prime \prime}=C_{s L^{3}}^{\prime \prime \prime}>0$ satisfying

$$
\frac{\alpha_{1} !}{\left(\alpha_{1}-\eta_{1}\right) !} \leq 2^{\left|\alpha_{1}\right|} \eta_{1} ! \leq 2^{\left|\alpha_{1}\right|} C_{s}^{\prime \prime \prime \prime} e^{s L^{3} \varphi^{*}\left(\frac{\left|\eta_{1}\right|}{s L^{3}}\right)} .
$$

We can assume $m \geq 0$ without loss of generality. By (0.4) and (0.1),

$$
\begin{aligned}
\omega(x, y, \xi) & \leq L \omega(x)+L \omega(y)+L \omega(\xi)+L \\
& \leq L^{2} \omega(y-x)+\left(L^{2}+L\right) \omega(y)+L \omega(\xi)+L^{2}+L .
\end{aligned}
$$


Therefore we obtain, with the previous estimates,

$$
\begin{aligned}
\left|D_{x}^{\alpha}\left(A_{1 / k, \chi}-A_{1 / l, \chi}\right)(f)(x)\right| & e^{2 n C_{1}} \sum_{\epsilon, \eta \in \mathbb{N}_{0}^{d}} e^{-n C_{1} \varphi^{*}\left(\frac{|\epsilon|}{n C_{1}}\right)} e^{-n C_{1} \varphi^{*}\left(\frac{|\eta|}{n C_{1}}\right)} \sum_{\begin{array}{c}
\alpha_{1}+\alpha_{2}+\alpha_{3}=\alpha \\
\epsilon_{1}+\epsilon_{2}+\epsilon_{3}=\epsilon \\
\eta_{1}+\eta_{2}+\eta_{3}=\eta, \eta_{1} \leq \alpha_{1}
\end{array}} \frac{\alpha !}{\alpha_{1} ! \alpha_{2} ! \alpha_{3} !} \frac{\epsilon !}{\epsilon_{1} ! \epsilon_{2} ! \epsilon_{3} !} \times \\
& \times \frac{\eta !}{\eta_{1} ! \eta_{2} ! \eta_{3} !} 2^{\left|\alpha_{1}\right|} C_{s}^{\prime \prime \prime \prime} e^{s L^{3} \varphi^{*}\left(\frac{\left|\eta_{1}\right|}{s L^{3}}\right)} \int\left|D_{x}^{\alpha_{3}} D_{\xi}^{\eta_{3}}\left(\chi\left(\frac{1}{k}(x, \xi)\right)-\chi\left(\frac{1}{l}(x, \xi)\right)\right)\right| \times \\
& \times\left(\int C_{3}^{2 n} e^{-n C_{2} \omega(\xi)} e^{\lambda L^{3} \varphi^{*}\left(\frac{\left|\alpha_{1}\right|}{\lambda L^{3}}\right)} e^{\lambda L^{3} \omega(\xi)} C_{s}^{\prime \prime} e^{s L^{3} \varphi^{*}\left(\frac{\left|\epsilon_{1}\right|}{s L^{3}}\right)} \times\right. \\
& \times e^{-n C_{2} \omega(y-x)} C_{s}^{\prime} e^{4 s L^{4}} e^{4 s L^{3} \varphi^{*}\left(\frac{\left|\alpha_{2}+\epsilon_{2}+\eta_{2}\right|}{4 s L^{3}}\right)} e^{m L^{2} \omega(y-x)} e^{m\left(L^{2}+L\right) \omega(y)} \times \\
& \left.\times e^{m L \omega(\xi)} e^{m L^{2}+m L} C_{s} e^{s L^{3} \varphi^{*}\left(\frac{\left|\epsilon_{3}\right|}{s L^{3}}\right)} e^{-s L^{3} \omega(y)} d y\right) d \xi .
\end{aligned}
$$

We take $n \in \mathbb{N}_{0}$ so that

$$
n C_{2} \geq \max \left\{1+\lambda L^{3}+m L, m L^{2}+(\lambda+L) L\right\} .
$$

Hence, in particular, we obtain

$$
e^{\left(-n C_{2}+m L+\lambda L^{3}\right) \omega(\xi)} \leq e^{-\omega(\xi)}
$$

Moreover, if we put $s \geq n C_{1}$ such that

$$
s L^{3} \geq m\left(L^{2}+L\right)+(\lambda+L) L+1,
$$

we have, by (0.1),

$$
\begin{aligned}
e^{m\left(L^{2}+L\right) \omega(y)} e^{-s L^{3} \omega(y)} e^{\left(m L^{2}-n C_{2}\right) \omega(y-x)} & \leq e^{-\omega(y)} e^{-(\lambda+L) L \omega(y)} e^{-(\lambda+L) L \omega(y-x)} \\
& \leq e^{-\omega(y)} e^{(\lambda+L) L} e^{-(\lambda+L) \omega(x)}
\end{aligned}
$$

Now, we assume $\left|\alpha_{3}+\eta_{3}\right|>0$. Then, there exists $C_{s}^{\prime \prime \prime \prime}=C_{4 s L^{3}}^{\prime \prime \prime \prime}>0$ such that, by the triangular inequality,

$$
\begin{aligned}
\mid D_{x}^{\alpha_{3}} & D_{\xi}^{\eta_{3}}\left(\chi\left(\frac{1}{k}(x, \xi)\right)-\chi\left(\frac{1}{l}(x, \xi)\right)\right) \mid \\
& \leq\left(\left(\frac{1}{k}\right)^{\left|\alpha_{3}+\eta_{3}\right|}+\left(\frac{1}{l}\right)^{\left|\alpha_{3}+\eta_{3}\right|}\right) C_{s}^{\prime \prime \prime \prime} e^{4 s L^{3} \varphi^{*}\left(\frac{\left|\alpha_{3}+\eta_{3}\right|}{4 s L^{3}}\right)} \\
& \leq\left(\frac{1}{k}+\frac{1}{l}\right) C_{s}^{\prime \prime \prime \prime \prime} e^{4 s L^{3} \varphi^{*}\left(\frac{\left|\alpha_{3}+\eta_{3}\right|}{4 s L^{3}}\right)}
\end{aligned}
$$


for $\left|\alpha_{3}+\eta_{3}\right|>0$. On the other hand, by the mean value theorem there exists $\zeta$ which lies in the line segment between $\frac{1}{l}(x, \xi)$ and $\frac{1}{k}(x, \xi)$ such that

$$
\left|\chi\left(\frac{1}{k}(x, \xi)\right)-\chi\left(\frac{1}{l}(x, \xi)\right)\right| \leq|\nabla \chi(\zeta)|\left|\frac{1}{k}-\frac{1}{l}\right||(x, \xi)| .
$$

It shows that, for some constant $C_{s}^{\prime \prime \prime \prime}>0$, as $|(x, \xi)| \leq\langle x\rangle\langle\xi\rangle$,

$$
\left|D_{x}^{\alpha_{3}} D_{\xi}^{\eta_{3}}\left(\chi\left(\frac{1}{k}(x, \xi)\right)-\chi\left(\frac{1}{l}(x, \xi)\right)\right)\right| \leq C_{s}^{\prime \prime \prime \prime} e^{4 s L^{3} \varphi^{*}\left(\frac{\left|\alpha_{3}+\eta_{3}\right|+1}{4 s L^{3}}\right)}\left|\frac{1}{k}+\frac{1}{l}\right|\langle x\rangle\langle\xi\rangle
$$

for all $\alpha_{3}, \eta_{3} \in \mathbb{N}_{0}^{d}$. Since $s \geq \lambda$, by $(0.11)$ (and the fact that $\varphi^{*}(t) / t$ is increasing) we have $4 s L^{3} \varphi^{*}\left(\frac{\left|\alpha_{3}+\eta_{3}\right|+1}{4 s L^{3}}\right) \leq \lambda \varphi^{*}\left(\frac{1}{\lambda}\right)+2 s L^{3} \varphi^{*}\left(\frac{\left|\alpha_{3}+\eta_{3}\right|}{2 s L^{3}}\right)$. Moreover, by Lemma 0.8 ,

$$
\begin{aligned}
& 2^{\left|\alpha_{1}\right|} e^{s L^{3} \varphi^{*}\left(\frac{\left|\eta_{1}\right|}{s L^{3}}\right)} e^{\lambda L^{3} \varphi^{*}\left(\frac{\left|\alpha_{1}\right|}{\lambda L^{3}}\right)} e^{s L^{3} \varphi^{*}\left(\frac{\left|\epsilon_{1}\right|}{s L^{3}}\right)} e^{4 s L^{3} \varphi^{*}\left(\frac{\left|\alpha_{2}+\epsilon_{2}+\eta_{2}\right|}{4 s L^{3}}\right)} \times \\
& \times e^{s L^{3} \varphi^{*}\left(\frac{\left|\epsilon_{3}\right|}{s L^{3}}\right)} e^{2 s L^{3} \varphi^{*}\left(\frac{\left|\alpha_{3}+\eta_{3}\right|}{2 s L^{3}}\right)} \leq e^{\lambda L^{3}} e^{\lambda L^{2} \varphi^{*}\left(\frac{|\alpha|}{\lambda L^{2}}\right)} e^{s L^{3} \varphi^{*}\left(\frac{|\epsilon|}{s L^{3}}\right)} e^{s L^{3} \varphi^{*}\left(\frac{|\eta|}{s L^{3}}\right) .}
\end{aligned}
$$

Furthermore, we obtain, by Lemma 0.1(4) and (0.10),

$$
\begin{aligned}
& \sum_{\substack{\alpha_{1}+\alpha_{2}+\alpha_{3}=\alpha \\
\epsilon_{1}+\epsilon_{3}+\epsilon_{3}=\epsilon \\
\eta_{1}+\eta_{2}+\eta_{3}=\eta, \eta_{1} \leq \alpha_{1}}} \frac{\alpha !}{\alpha_{1} ! \alpha_{2} ! \alpha_{3} !} \frac{\epsilon !}{\epsilon_{1} ! \epsilon_{2} ! \epsilon_{3} !} \frac{\eta !}{\eta_{1} ! \eta_{2} ! \eta_{3} !} e^{\lambda L^{2} \varphi^{*}\left(\frac{|\alpha|}{\lambda L^{2}}\right)} e^{s L^{3} \varphi^{*}\left(\frac{|\epsilon|}{s L^{3}}\right)} e^{s L^{3} \varphi^{*}\left(\frac{|\eta|}{s L^{3}}\right)} \\
& \begin{array}{l}
\leq e^{2|\alpha+\epsilon+\eta|} e^{\lambda L^{2} \varphi^{*}\left(\frac{|\alpha|}{\lambda L^{2}}\right)} e^{s L^{3} \varphi^{*}\left(\frac{|\epsilon|}{s L^{3}}\right)} e^{s L^{3} \varphi^{*}\left(\frac{|\eta|}{s L^{3}}\right)} \\
\leq e^{(\lambda+2 s L)\left(L+L^{2}\right)} e^{\lambda \varphi^{*}\left(\frac{|\alpha|}{\lambda}\right)} e^{s L \varphi^{*}\left(\frac{|\epsilon|}{s L}\right)} e^{s L \varphi^{*}\left(\frac{|\eta|}{s L}\right)} .
\end{array}
\end{aligned}
$$

As the selection of $n$ and $s$ depends on $\lambda$, we write

$$
\begin{aligned}
D_{\lambda}= & C_{s} C_{s}^{\prime} C_{s}^{\prime \prime} C_{s}^{\prime \prime \prime} C_{s}^{\prime \prime \prime \prime} e^{4 s L^{4}} e^{(\lambda+2 s L)\left(L+L^{2}\right)} e^{m L^{2}+m L} \times \\
& \times e^{(\lambda+L) L+\lambda L^{3}} e^{\lambda \varphi^{*}\left(\frac{1}{\lambda}\right)} C_{3}^{2 n} e^{2 n C_{1}}>0,
\end{aligned}
$$

and hence,

$$
\begin{aligned}
& \left|D_{x}^{\alpha}\left(A_{1 / k, \chi}-A_{1 / l, \chi}\right)(f)(x)\right| \\
& \leq D_{\lambda}\left|\frac{1}{k}+\frac{1}{l}\right| e^{\lambda \varphi^{*}\left(\frac{|\alpha|}{\lambda}\right)} \sum_{\epsilon, \eta \in \mathbb{N}_{0}^{d}} e^{-n C_{1} \varphi^{*}\left(\frac{|\epsilon|}{n C_{1}}\right)} e^{-n C_{1} \varphi^{*}\left(\frac{|\eta|}{n C_{1}}\right)} \times \\
& \quad \times e^{s L \varphi^{*}\left(\frac{|\epsilon|}{s L}\right)} e^{s L \varphi^{*}\left(\frac{|\eta|}{s L}\right)}\langle x\rangle e^{-(\lambda+L) \omega(x)}\left(\int e^{-\omega(y)} d y\right)\left(\int\langle\xi\rangle e^{-\omega(\xi)} d \xi\right) .
\end{aligned}
$$


We use (0.7) and (0.6) to obtain

$$
\langle x\rangle \leq e^{\varphi^{*}(1)} e^{\omega(\langle x\rangle)} \leq e^{\varphi^{*}(1)} e^{L} e^{L \omega(x)} .
$$

Similarly,

$$
\langle\xi\rangle \leq e^{\frac{1}{2 L} \varphi^{*}(2 L)} e^{\frac{1}{2 L} \omega(\langle\xi\rangle)} \leq e^{\frac{1}{2 L} \varphi^{*}(2 L)} e^{\frac{1}{2}} e^{\frac{1}{2} \omega(\xi)},
$$

and thus the integrals converge by condition $(\gamma)$ of the weight $\omega$. For the convergence of the series, we treat the sum in $\epsilon$ (the other one will follow in the same way). That is, we need to estimate

$$
\sum_{\epsilon \in \mathbb{N}_{0}^{d}} e^{-n C_{1} \varphi^{*}\left(\frac{|\epsilon|}{n C_{1}}\right)} e^{s L \varphi^{*}\left(\frac{|\epsilon|}{s L}\right)} .
$$

We have by $(0.10)$, as $s \geq n C_{1}$,

$$
\begin{aligned}
e^{-n C_{1} \varphi^{*}\left(\frac{|\epsilon|}{n C_{1}}\right)} e^{s L \varphi^{*}\left(\frac{|\epsilon|}{s L}\right)} & =e^{-|\epsilon|} e^{-n C_{1} \varphi^{*}\left(\frac{|\epsilon|}{n C_{1}}\right)} e^{|\epsilon|+s L \varphi^{*}\left(\frac{|\epsilon|}{s L}\right)} \\
& \leq e^{-|\epsilon|} e^{-n C_{1} \varphi^{*}\left(\frac{|\epsilon|}{n C_{1}}\right)} e^{s L} e^{s \varphi^{*}\left(\frac{|\epsilon|}{s}\right)} \leq e^{s L} e^{-|\epsilon|} .
\end{aligned}
$$

The series $\sum_{\epsilon \in \mathbb{N}_{0}^{d}} e^{-|\epsilon|}$ is convergent (by Lemma 0.1(8)), and also (1.14). Hence, from (1.13) we show that for all $\lambda>0$ formula (1.9) holds and the result then follows.

Lemma 1.10. Given $a(x, y, \xi) \in \mathrm{GA}_{\rho}^{m, \omega}$ and $f \in \mathcal{S}_{\omega}\left(\mathbb{R}^{d}\right)$, for all $\lambda>0$ there exists $C_{\lambda}>0$ such that for all $x, \xi \in \mathbb{R}^{d}$,

$$
\left|\int_{\mathbb{R}^{d}} e^{i(x-y) \cdot \xi} a(x, y, \xi) f(y) d y\right| \leq C_{\lambda} e^{-\lambda \omega(\xi)} e^{\max \left\{m, m L^{2}\right\} \omega(x)} .
$$

Proof. As in Proposition 1.9, we integrate by parts in the integrand of the lefthand side of (1.15) with formula (1.6) for a suitable $n \in \mathbb{N}$ to be determined. We have

$$
\begin{aligned}
& e^{i(x-y) \cdot \xi} \frac{1}{G^{n}(\xi)} G^{n}\left(D_{y}\right)(a(x, y, \xi) f(y)) \\
& =e^{i(x-y) \cdot \xi} \frac{1}{G^{n}(\xi)} \sum_{\eta \in \mathbb{N}_{0}^{d}} b_{\eta} \sum_{\eta_{1}+\eta_{2}=\eta} \frac{\eta !}{\eta_{1} ! \eta_{2} !} D_{y}^{\eta_{1}} a(x, y, \xi) D_{y}^{\eta_{2}} f(y),
\end{aligned}
$$

and therefore

$$
\begin{aligned}
& \int_{\mathbb{R}^{d}} e^{i(x-y) \cdot \xi} a(x, y, \xi) f(y) d y \\
& \quad=\sum_{\eta \in \mathbb{N}_{0}^{d}} b_{\eta} \sum_{\eta_{1}+\eta_{2}=\eta} \frac{\eta !}{\eta_{1} ! \eta_{2} !} \frac{1}{G^{n}(\xi)} \int_{\mathbb{R}^{d}} e^{i(x-y) \cdot \xi} D_{y}^{\eta_{1}} a(x, y, \xi) D_{y}^{\eta_{2}} f(y) d y
\end{aligned}
$$


We fix $\lambda>0$ and we take $s \geq \lambda$, to be determined. Similarly as in (1.11), from Definition 1.3 for that $s>0$ there exists $C_{s}=C_{s L^{3}}>0$ such that

$$
\begin{aligned}
\left|D_{y}^{\eta_{1}} a(x, y, \xi)\right| & \leq C_{s}\left(\frac{\langle x-y\rangle}{\langle(x, y, \xi)\rangle}\right)^{\rho\left|\eta_{1}\right|} e^{s L^{3} \rho \varphi^{*}\left(\frac{\left|\eta_{1}\right|}{s L^{3}}\right)} e^{m \omega(x, y, \xi)} \\
& \leq C_{s} e^{s L^{3}} e^{s L^{2} \varphi^{*}\left(\frac{\left|\eta_{1}\right|}{s L^{2}}\right)} e^{m \omega(x, y, \xi)}
\end{aligned}
$$

Since $f \in \mathcal{S}_{\omega}\left(\mathbb{R}^{d}\right)$ there exists $C_{s}^{\prime}=C_{s L^{2}}^{\prime}>0$ such that

$$
\left|D_{y}^{\eta_{2}} f(y)\right| \leq C_{s}^{\prime} e^{s L^{2} \varphi^{*}\left(\frac{\left|\eta_{2}\right|}{s L^{2}}\right)} e^{-s L^{2} \omega(y)} .
$$

Again, by Corollaries 0.23 and 0.20 there are $C_{1}, C_{2}, C_{3}>0$ such that

$$
\left|b_{\eta}\right| \leq e^{n C_{1}} e^{-n C_{1} \varphi^{*}\left(\frac{|\eta|}{n C_{1}}\right)}, \quad\left|\frac{1}{G^{n}(\xi)}\right| \leq C_{3}^{n} e^{-n C_{2} \omega(\xi)} .
$$

We then obtain that the left-hand side of (1.15) is estimated by

$$
\begin{aligned}
& C_{3}^{n} e^{n C_{1}} e^{-n C_{2} \omega(\xi)} C_{s} C_{s}^{\prime} e^{s L^{3}}\left(\int_{\mathbb{R}^{d}} e^{m \omega(x, y, \xi)} e^{-s L^{2} \omega(y)} d y\right) \times \\
& \quad \times \sum_{\eta \in \mathbb{N}_{0}^{d}} e^{-n C_{1} \varphi^{*}\left(\frac{|\eta|}{n C_{1}}\right)} \sum_{\eta_{1}+\eta_{2}=\eta} \frac{\eta !}{\eta_{1} ! \eta_{2} !} e^{s L^{2} \varphi^{*}\left(\frac{\left|\eta_{1}\right|}{s L^{2}}\right)} e^{s L^{2} \varphi^{*}\left(\frac{\left|\eta_{2}\right|}{s L^{2}}\right)} .
\end{aligned}
$$

From (0.4), we have

$$
\omega(x) \leq \omega(x, y, \xi) \leq L \omega(x)+L \omega(y)+L \omega(\xi)+L .
$$

We consider $m \geq 0$. We take $n \in \mathbb{N}_{0}$ so that $n C_{2} \geq \lambda+m L$. Then

$$
e^{-n C_{2} \omega(\xi)} e^{m L \omega(\xi)} \leq e^{-\lambda \omega(\xi)} .
$$

Moreover, if we take $s \geq n C_{1}$ such that

$$
s L^{2} \geq 1+m L,
$$

we guarantee the convergence of the integral as $e^{-s L^{2} \omega(y)} e^{m L \omega(y)} \leq e^{-\omega(y)}$, by condition $(\gamma)$. On the other hand, it follows from Lemma 0.8 that

$$
\sum_{\eta_{1}+\eta_{2}=\eta} \frac{\eta !}{\eta_{1} ! \eta_{2} !} e^{s L^{2} \varphi^{*}\left(\frac{\left|\eta_{1}\right|}{s L^{2}}\right)} e^{s L^{2} \varphi^{*}\left(\frac{\left|\eta_{2}\right|}{s L^{2}}\right)} \leq e^{s L^{2}} e^{s L \varphi^{*}\left(\frac{|\eta|}{s L}\right)} .
$$

Since $s \geq n C_{1}$, the series depending on $\eta$ in (1.16) is convergent as it is proved in (1.14). Both $n$ and $s$ depend on $\lambda>0$, therefore the estimate (1.15) holds. 
Lemma 1.11. For $a(x, y, \xi) \in \mathrm{GA}_{\rho}^{m, \omega}$ and $\chi \in \mathcal{S}_{\omega}\left(\mathbb{R}^{2 d}\right)$, we denote

$$
K(x, y):=\int_{\mathbb{R}^{d}} e^{i(x-y) \cdot \xi} a(x, y, \xi) \chi(x, \xi) d \xi
$$

We have

(a) $K(x, y) \in \mathcal{S}_{\omega}\left(\mathbb{R}^{2 d}\right)$.

(b) The operator $T: \mathcal{S}_{\omega}\left(\mathbb{R}^{d}\right) \rightarrow \mathcal{S}_{\omega}\left(\mathbb{R}^{d}\right)$ given by $T(f)(x)=\int K(x, y) f(y) d y$, $x \in \mathbb{R}^{d}$, is linear and continuous.

Proof. (a) Differentiating $K(x, y)$ one obtains

$$
\begin{aligned}
D_{x}^{\alpha} D_{y}^{\beta} K(x, y)= & \sum_{\substack{\alpha_{1}+\alpha_{2}+\alpha_{3}=\alpha \\
\beta_{1}+\beta_{2}=\beta}} \frac{\alpha !}{\alpha_{1} ! \alpha_{2} ! \alpha_{3} !} \frac{\beta !}{\beta_{1} ! \beta_{2} !}(-1)^{\left|\beta_{1}\right|} \times \\
& \times \int_{\mathbb{R}^{d}} e^{i(x-y) \cdot \xi} \xi^{\alpha_{1}+\beta_{1}} D_{x}^{\alpha_{2}} D_{y}^{\beta_{2}} a(x, y, \xi) D_{x}^{\alpha_{3}} \chi(x, \xi) d \xi,
\end{aligned}
$$

for all $\alpha, \beta \in \mathbb{N}_{0}^{d}, x, y \in \mathbb{R}^{d}$. We integrate by parts via formula (1.7) for an appropriate power $n \in \mathbb{N}$. Then, the integrand in (1.17) is equal to

$$
\begin{aligned}
& e^{i(x-y) \cdot \xi} \frac{1}{G^{n}(y-x)} G^{n}\left(D_{\xi}\right)\left(\xi^{\alpha_{1}+\beta_{1}} D_{x}^{\alpha_{2}} D_{y}^{\beta_{2}} a(x, y, \xi) D_{x}^{\alpha_{3}} \chi(x, \xi)\right) \\
& =e^{i(x-y) \cdot \xi} \frac{1}{G^{n}(y-x)} \sum_{\eta \in \mathbb{N}_{0}^{d}} b_{\eta} \sum_{\eta_{1}+\eta_{2}+\eta_{3}=\eta, \eta_{1} \leq \alpha_{1}+\beta_{1}} \frac{\eta !}{\eta_{1} ! \eta_{2} ! \eta_{3} !} \frac{\left(\alpha_{1}+\beta_{1}\right) !}{\left(\alpha_{1}+\beta_{1}-\eta_{1}\right) !} \times \\
& \quad \times \xi^{\alpha_{1}+\beta_{1}-\eta_{1}} D_{x}^{\alpha_{2}} D_{y}^{\beta_{2}} D_{\xi}^{\eta_{2}} a(x, y, \xi) D_{x}^{\alpha_{3}} D_{\xi}^{\eta_{3}} \chi(x, \xi),
\end{aligned}
$$

and this yields from $(1.17)$ that $D_{x}^{\alpha} D_{y}^{\beta} K(x, y)$ is equal to

$$
\begin{aligned}
& \frac{1}{G^{n}(y-x)} \sum_{\eta \in \mathbb{N}_{0}^{d}} b_{\eta} \sum_{\substack{\alpha_{1}+\alpha_{2}+\alpha_{3}=\alpha \\
\beta_{1}+\beta_{2}=\beta \\
\eta_{1}+\eta_{2}+\eta_{3}=\eta, \eta_{1} \leq \alpha_{1}+\beta_{1}}} \frac{\alpha !}{\alpha_{1} ! \alpha_{2} ! \alpha_{3} !} \frac{\beta !}{\beta_{1} ! \beta_{2} !} \frac{\eta !}{\eta_{1} ! \eta_{2} ! \eta_{3} !} \frac{\left(\alpha_{1}+\beta_{1}\right) !}{\left(\alpha_{1}+\beta_{1}-\eta_{1}\right) !} \times \\
& \times(-1)^{\left|\beta_{1}\right|} \int_{\mathbb{R}^{d}} e^{i(x-y) \cdot \xi} \xi^{\alpha_{1}+\beta_{1}-\eta_{1}} D_{x}^{\alpha_{2}} D_{y}^{\beta_{2}} D_{\xi}^{\eta_{2}} a(x, y, \xi) D_{x}^{\alpha_{3}} D_{\xi}^{\eta_{3}} \chi(x, \xi) d \xi
\end{aligned}
$$

We fix $\lambda>0$ and we take $s \geq \lambda$. Since $\chi \in \mathcal{S}_{\omega}\left(\mathbb{R}^{2 d}\right)$, for that $s>0$ there exists $C_{s}=C_{2 s L^{3}}>0$ so that

$$
\left|D_{x}^{\alpha_{3}} D_{\xi}^{\eta_{3}} \chi(x, \xi)\right| \leq C_{s} e^{2 s L^{3} \varphi^{*}\left(\frac{\left|\alpha_{3}+\eta_{3}\right|}{2 s L^{3}}\right)} e^{-2 s L^{3} \omega(x, \xi)} .
$$


By Definition 1.3 there exists $C_{s}^{\prime}=C_{2 s L^{4}}^{\prime}>0$ such that (as in (1.11))

$$
\begin{aligned}
\left|D_{x}^{\alpha_{2}} D_{y}^{\beta_{2}} D_{\xi}^{\eta_{2}} a(x, y, \xi)\right| & \leq C_{s}^{\prime}\left(\frac{\langle x-y\rangle}{\langle(x, y, \xi)\rangle}\right)^{\rho\left|\alpha_{2}+\beta_{2}+\eta_{2}\right|} e^{2 s L^{4} \rho \varphi^{*}\left(\frac{\left|\alpha_{2}+\beta_{2}+\eta_{2}\right|}{2 s L^{4}}\right)} e^{m \omega(x, y, \xi)} \\
& \leq C_{s}^{\prime} e^{2 s L^{4}} e^{2 s L^{3} \varphi^{*}\left(\frac{\left|\alpha_{2}+\beta_{2}+\eta_{2}\right|}{2 s L^{3}}\right)} e^{m \omega(x, y, \xi)} .
\end{aligned}
$$

Formula (0.7) gives (if $|\xi| \geq 1$ )

$$
|\xi|^{\left|\alpha_{1}+\beta_{1}-\eta_{1}\right|} \leq e^{\lambda L^{3} \varphi^{*}\left(\frac{\left|\alpha_{1}+\beta_{1}-\eta_{1}\right|}{\lambda L^{3}}\right)} e^{\lambda L^{3} \omega(\xi)} .
$$

By (0.12) there exists $C_{s}^{\prime \prime}=C_{s L^{3}}^{\prime \prime}>0$ so that

$$
\frac{\left(\alpha_{1}+\beta_{1}\right) !}{\left(\alpha_{1}+\beta_{1}-\eta_{1}\right) !} \leq 2^{\left|\alpha_{1}+\beta_{1}\right|} \eta_{1} ! \leq 2^{\left|\alpha_{1}+\beta_{1}\right|} C_{s}^{\prime \prime} e^{s L^{3} \varphi^{*}\left(\frac{\left|\eta_{1}\right|}{s L^{3}}\right)},
$$

and by (0.10), we have

$$
2^{\left|\alpha_{1}+\beta_{1}\right|} e^{\lambda L^{3} \varphi^{*}\left(\frac{\left|\alpha_{1}+\beta_{1}\right|}{\lambda L^{3}}\right)} \leq e^{\lambda L^{2} \varphi^{*}\left(\frac{\left|\alpha_{1}+\beta_{1}\right|}{\lambda L^{2}}\right)} e^{\lambda L^{3}} .
$$

On the other hand, by Corollaries 0.23 and 0.20 there are $C_{1}, C_{2}, C_{3}>0$ such that

$$
\left|b_{\eta}\right| \leq e^{n C_{1}} e^{-n C_{1} \varphi^{*}\left(\frac{|\eta|}{n C_{1}}\right)}, \quad\left|\frac{1}{G^{n}(y-x)}\right| \leq C_{3}^{n} e^{-n C_{2} \omega(y-x)} .
$$

Hence, we estimate $\left|D_{x}^{\alpha} D_{y}^{\beta} K(x, y)\right|$ by

$$
\begin{gathered}
C_{s} C_{s}^{\prime} C_{s}^{\prime \prime} e^{2 s L^{4}} e^{\lambda L^{3}} C_{3}^{n} e^{n C_{1}} e^{-n C_{2} \omega(y-x)}\left(\int_{\mathbb{R}^{d}} e^{\lambda L^{3} \omega(\xi)} e^{-2 s L^{3} \omega(x, \xi)} e^{m \omega(x, y, \xi)} d \xi\right) \times \\
\times \sum_{\eta \in \mathbb{N}_{0}^{d}} e^{-n C_{1} \varphi^{*}\left(\frac{|\eta|}{n C_{1}}\right)} \sum_{\substack{\alpha_{1}+\alpha_{2}+\alpha_{3}=\alpha \\
\beta_{1}+\beta_{2}=\beta}} \frac{\alpha !}{\alpha_{1} ! \alpha_{2} ! \alpha_{3} !} \frac{\beta !}{\beta_{1} ! \beta_{2} !} \frac{\eta !}{\eta_{1} ! \eta_{2} ! \eta_{3} !} \times \\
\times e^{s L^{3} \varphi^{*}\left(\frac{\left|\eta_{1}\right|}{s L^{3}}\right)} e^{\lambda L^{2} \varphi^{*}\left(\frac{\left|\alpha_{1}+\beta_{1}-\eta_{1}\right|}{\lambda L^{2}}\right)} e^{2 s L^{3} \varphi^{*}\left(\frac{\left|\alpha_{2}+\beta_{2}+\eta_{2}\right|}{2 s L^{3}}\right)} e^{2 s L^{3} \varphi^{*}\left(\frac{\left|\alpha_{3}+\eta_{3}\right|}{2 s L^{3}}\right)} .
\end{gathered}
$$

First of all we deduce from (0.3) and (0.1), for $m \geq 0$ (if $m<0$ the proof is easier)

$$
\begin{aligned}
m \omega(x, y, \xi) & \leq m L(\omega(x, \xi)+\omega(y)+1) \\
& \leq m L(\omega(x, \xi)+L \omega(y-x)+L \omega(x)+1+L) .
\end{aligned}
$$

We take $n \in \mathbb{N}_{0}$ satisfying

$$
n C_{2} \geq m L^{2}+\lambda L^{2}
$$


Thus, by (0.1),

$$
e^{-n C_{2} \omega(y-x)} e^{m L^{2} \omega(y-x)} \leq e^{-\lambda L^{2} \omega(y-x)} \leq e^{-\lambda L \omega(y)} e^{\lambda L^{2} \omega(x)} e^{\lambda L^{2}} .
$$

Now, we take $s \geq n C_{1}$ so that

$$
2 s L^{3} \geq 2\left(\lambda L^{3}+\lambda L+m L^{2}\right)+m L .
$$

Therefore,

$$
e^{\left(-2 s L^{3}+m L\right) \omega(x, \xi)} \leq e^{-2\left(\lambda L^{3}+\lambda L+m L^{2}\right) \omega(x, \xi)} \leq e^{-\left(\lambda L^{3}+\lambda\right) \omega(\xi)} e^{-\left(\lambda L^{2}+\lambda L+m L^{2}\right) \omega(x)} .
$$

By (1.19), (1.20), and (1.21) we obtain, from (0.3),

$$
\begin{aligned}
e^{-n C_{2} \omega(y-x)} e^{\lambda L^{3} \omega(\xi)} e^{-2 s L^{3} \omega(x, \xi)} e^{m \omega(x, y, \xi)} & \leq e^{-\lambda L \omega(y)} e^{-\lambda L \omega(x)} e^{-\lambda \omega(\xi)} e^{\lambda L^{2}} e^{m L+m L^{2}} \\
& \leq e^{\lambda L} e^{-\lambda \omega(x, y)} e^{-\lambda \omega(\xi)} e^{\lambda L^{2}} e^{m L+m L^{2}} .
\end{aligned}
$$

The integral converges by condition $(\gamma)$ of the weight $\omega$. On the other hand, since $s \geq \lambda$ we obtain, by Lemma 0.8 ,

$$
\begin{aligned}
& e^{s L^{3} \varphi^{*}\left(\frac{\left|\eta_{1}\right|}{s L^{3}}\right)} e^{\lambda L^{2} \varphi^{*}\left(\frac{\left|\alpha_{1}+\beta_{1}-\eta_{1}\right|}{\lambda L^{2}}\right)} e^{2 s L^{3} \varphi^{*}\left(\frac{\left|\alpha_{2}+\beta_{2}+\eta_{2}\right|}{2 s L^{3}}\right)} e^{2 s L^{3} \varphi^{*}\left(\frac{\left|\alpha_{3}+\eta_{3}\right|}{2 s L^{3}}\right)} \\
& \leq e^{\lambda L^{2} \varphi^{*}\left(\frac{|\alpha+\beta|}{\lambda L^{2}}\right)} e^{s L^{3} \varphi^{*}\left(\frac{|\eta|}{s L^{3}}\right)} .
\end{aligned}
$$

As

$$
\sum_{\substack{\alpha_{1}+\alpha_{2}+\alpha_{3}=\alpha \\ \beta_{1}+\beta_{2}=\beta \\ \eta_{1}+\eta_{2}+\eta_{3}=\eta}} \frac{\alpha !}{\alpha_{1} ! \alpha_{2} ! \alpha_{3} !} \frac{\beta !}{\beta_{1} ! \beta_{2} !} \frac{\eta !}{\eta_{1} ! \eta_{2} ! \eta_{3} !}=3^{|\alpha+\eta|} 2^{|\beta|} \leq e^{2|\alpha+\beta+\eta|},
$$

we use formula (0.10) to get

$$
e^{2|\alpha+\beta|} e^{\lambda L^{2} \varphi^{*}\left(\frac{|\alpha+\beta|}{\lambda L^{2}}\right)} e^{2|\eta|} e^{s L^{3} \varphi^{*}\left(\frac{|\eta|}{s L^{3}}\right)} \leq e^{\lambda L^{2}+\lambda L} e^{\lambda \varphi^{*}\left(\frac{|\alpha+\beta|}{\lambda}\right)} e^{s L^{3}+s L^{2}} e^{s L \varphi^{*}\left(\frac{|\eta|}{s L}\right)} .
$$

The series depending on $\eta$ in (1.18) converges as in (1.14). Since $n$ and $s$ depend on $\lambda>0$, there exists $C_{\lambda}>0$ such that

$$
\left|D_{x}^{\alpha} D_{y}^{\beta} K(x, y)\right| \leq C_{\lambda} e^{\lambda \varphi^{*}\left(\frac{|\alpha+\beta|}{\lambda}\right)} e^{-\lambda \omega(x, y)}, \quad \alpha, \beta \in \mathbb{N}_{0}^{d}, x, y \in \mathbb{R}^{d} .
$$

(b) We note that for $f \in \mathcal{S}_{\omega}\left(\mathbb{R}^{d}\right)$, as $\varphi^{*}(0)=0$, we have, for any $\mu>0$,

$$
\sup _{y \in \mathbb{R}^{d}}|f(y)| \leq \sup _{y \in \mathbb{R}^{d}}|f(y)| e^{\mu \omega(y)} \leq \sup _{\alpha \in \mathbb{N}_{0}^{d}} \sup _{y \in \mathbb{R}^{d}}\left|D^{\alpha} f(y)\right| e^{-\mu \varphi^{*}\left(\frac{|\alpha|}{\mu}\right)} e^{\mu \omega(y)}=|f|_{\mu},
$$


being $|\cdot|_{\mu}$ the seminorm in (0.14). Now, to prove that $T$ is continuous, we differentiate under the integral sign the function $T(f)$, and by (a) we obtain that for all $\lambda>0$ there exists $C_{\lambda}=C_{2 \lambda}>0$ such that

$$
\begin{aligned}
\left|D^{\alpha} T(f)(x)\right| & \leq \int_{\mathbb{R}^{d}}\left|D_{x}^{\alpha} K(x, y)\right||f(y)| d y \\
& \leq C_{\lambda} e^{2 \lambda \varphi^{*}\left(\frac{|\alpha|}{2 \lambda}\right)} \int_{\mathbb{R}^{d}} e^{-2 \lambda \omega(x, y)}|f(y)| d y \\
& \leq C_{\lambda} e^{\lambda \varphi^{*}\left(\frac{|\alpha|}{\lambda}\right)} e^{-\lambda \omega(x)}|f|_{\mu} \int_{\mathbb{R}^{d}} e^{-\lambda \omega(y)} d y
\end{aligned}
$$

for all $\alpha \in \mathbb{N}_{0}^{d}, x \in \mathbb{R}^{d}$, and for any $\mu>0$. This gives the conclusion.

Lemma 1.12. Every global amplitude is an $\omega$-temperate ultradistribution in $\mathcal{S}_{\omega}^{\prime}\left(\mathbb{R}^{3 d}\right)$.

Proof. Since $a \in \mathrm{GA}_{\rho}^{m, \omega}$ is a $C^{\infty}\left(\mathbb{R}^{3 d}\right)$ function, we have

$$
\langle a(x, y, \xi), f(x, y, \xi)\rangle=\iiint a(x, y, \xi) f(x, y, \xi) d x d y d \xi, \quad f \in \mathcal{S}_{\omega}\left(\mathbb{R}^{3 d}\right) .
$$

Again since $a \in \mathrm{GA}_{\rho}^{m, \omega}$, there exists $C>0$ such that

$$
\begin{aligned}
|\langle a, f\rangle| & \leq \iiint|a(x, y, \xi)||f(x, y, \xi)| d x d y d \xi \\
& \leq C \iiint e^{m \omega(x, y, \xi)}|f(x, y, \xi)| d x d y d \xi \\
& \leq C\left(\iiint e^{-\omega(x, y, \xi)} d x d y d \xi\right) \sup _{x, y, \xi \in \mathbb{R}^{d}}|f(x, y, \xi)| e^{(|m|+1) \omega(x, y, \xi)} \\
& \leq C^{\prime}|f|_{|m|+1}
\end{aligned}
$$

for all $f \in \mathcal{S}_{\omega}\left(\mathbb{R}^{3 d}\right)$, where $C^{\prime}=C \iiint e^{-\omega(x, y, \xi)} d x d y d \xi>0$ and $|f|_{|m|+1}$ is as in (0.14). Hence $a \in \mathcal{S}_{\omega}^{\prime}\left(\mathbb{R}^{3 d}\right)$.

Since $\mathcal{S}_{\omega}\left(\mathbb{R}^{2 d}\right)$ is nuclear, there exists a kernel $K_{A}$ for the pseudodifferential operator $A$. If an amplitude $a(x, y, \xi)$ belongs to $\mathcal{S}_{\omega}\left(\mathbb{R}^{3 d}\right)$, then by Fubini's theorem,

$$
\left\langle K_{A}(x, y), \chi(x, y)\right\rangle=\iint\left(\int e^{i(x-y) \cdot \xi} a(x, y, \xi) d \xi\right) \chi(x, y) d x d y, \quad \chi \in \mathcal{S}_{\omega}\left(\mathbb{R}^{2 d}\right) .
$$


Then, the kernel $K_{A}$ is given formally, as in [64, (23.22)], by

$$
K_{A}(x, y)=\int e^{i(x-y) \cdot \xi} a(x, y, \xi) d \xi,
$$

which defines an ultradistribution in $\mathcal{S}_{\omega}^{\prime}\left(\mathbb{R}^{2 d}\right)$.

Moreover, we can also characterize the global symbols in $\mathcal{S}_{\omega}\left(\mathbb{R}^{2 d}\right)$ in terms of the kernel of the associated pseudodifferential operator (cf. [55, Proposition 1.2.1]):

Corollary 1.13. A global symbol $a(x, \xi)$ belongs to $\mathcal{S}_{\omega}\left(\mathbb{R}^{2 d}\right)$ if and only if

$$
K(x, y):=\int_{\mathbb{R}^{d}} e^{i(x-y) \cdot \xi} a(x, \xi) d \xi
$$

is in $\mathcal{S}_{\omega}\left(\mathbb{R}^{2 d}\right)$.

Proof. As the function equivalent to 1 in $\mathbb{R}^{3 d}$ is a global amplitude in $\mathrm{GA}_{\rho}^{0, \omega}$, the necessity follows by Lemma 1.11(a). On the other hand, when the kernel belongs to $\mathcal{S}_{\omega}\left(\mathbb{R}^{2 d}\right)$, a can be written as

$$
a(x, \xi)=(2 \pi)^{-d} \mathcal{F}_{y \mapsto \xi} K(x, x-y) .
$$

Indeed, by (0.34),

$$
\begin{aligned}
\mathcal{F}_{y \mapsto \xi} K(x, x-y) & =\int_{\mathbb{R}^{d}} e^{-i y \cdot \xi} K(x, x-y) d y=\int_{\mathbb{R}^{d}} e^{-i y \cdot \xi}\left(\int_{\mathbb{R}^{d}} e^{i y \cdot \xi} a(x, \xi) d \xi\right) d y \\
& =(2 \pi)^{d} \int_{\mathbb{R}^{d}} e^{-i y \cdot \xi} \mathcal{F}_{\xi \mapsto y}^{-1} a(x, y) d y=(2 \pi)^{d} a(x, \xi) .
\end{aligned}
$$

This shows the result since $\mathcal{S}_{\omega}\left(\mathbb{R}^{2 d}\right)$ is invariant by partial Fourier transform (see e.g. [13, Remark 4.10]).

Remark 1.14. If $\chi \in \mathcal{S}_{\omega}\left(\mathbb{R}^{d}\right)$ only depends on the variable $\xi$, we do not get Lemma 1.11(a), but the following: $K \in C^{\infty}\left(\mathbb{R}^{2 d}\right)$, and for every $\lambda>0$ there exists $C_{\lambda}>0$ such that

$$
\left|D_{x}^{\alpha} D_{y}^{\beta} K(x, y)\right| \leq C_{\lambda} e^{\lambda \varphi^{*}\left(\frac{|\alpha+\beta|}{\lambda}\right)} e^{\max \left\{m, m L^{2}\right\} \omega(y)}, \quad \alpha, \beta \in \mathbb{N}_{0}^{d}, x, y \in \mathbb{R}^{d} .
$$

Indeed, $\left|D_{x}^{\alpha} D_{y}^{\beta} K(x, y)\right|$ is estimated as in (1.18), replacing $e^{-2 s L^{3} \omega(x, \xi)}$ with $e^{-2 s L^{3} \omega(\xi)}$, and $\eta_{3}$ is now zero. Using (0.4), it is enough to take $n$ and $s$ as in the proof of Lemma 1.11(a) to obtain the estimate above.

However, under this weaker estimate on K, Lemma 1.11(b) is still true. 
Theorem 1.15. The operator $A: \mathcal{S}_{\omega}\left(\mathbb{R}^{d}\right) \rightarrow \mathcal{S}_{\omega}\left(\mathbb{R}^{d}\right)$ given by (1.5) is well defined, linear, and continuous.

Proof. We fix $\chi \in \mathcal{S}_{\omega}\left(\mathbb{R}^{2 d}\right)$ so that $\chi(0)=1$. For every $f \in \mathcal{S}_{\omega}\left(\mathbb{R}^{d}\right)$, the sequence $\left\{A_{1 / n, \chi}(f)\right\}_{n}$ in (1.4) converges in $\mathcal{S}_{\omega}\left(\mathbb{R}^{d}\right)$ by Proposition 1.9. Moreover, the operator $A_{1 / n, \chi}: \mathcal{S}_{\omega}\left(\mathbb{R}^{d}\right) \rightarrow \mathcal{S}_{\omega}\left(\mathbb{R}^{d}\right)$ is linear, and, by Lemma 1.11, is well defined and continuous for every $n \in \mathbb{N}$. We denote by $A_{\chi}$ the operator given by the limit:

$$
A_{\chi}(f):=\lim _{n \rightarrow \infty} \iint_{\mathbb{R}^{d} \times \mathbb{R}^{d}} e^{i(x-y) \cdot \xi} a(x, y, \xi) f(y) \chi\left(\frac{1}{n}(x, \xi)\right) d y d \xi, \quad f \in \mathcal{S}_{\omega}\left(\mathbb{R}^{d}\right) .
$$

By Proposition 1.9, this operator is well defined from $\mathcal{S}_{\omega}\left(\mathbb{R}^{d}\right)$ to $\mathcal{S}_{\omega}\left(\mathbb{R}^{d}\right)$. Since $\mathcal{S}_{\omega}\left(\mathbb{R}^{d}\right)$ is barrelled, the family $\left\{A_{1 / n, \chi}\right\}_{n}$ is equicontinuous by BanachSteinhaus theorem. Then, for every seminorm $p$ of $\mathcal{S}_{\omega}\left(\mathbb{R}^{d}\right)$, there exist $C>0$ and another seminorm $q$ of $\mathcal{S}_{\omega}\left(\mathbb{R}^{d}\right)$ such that $p\left(A_{1 / n, \chi}(f)\right) \leq C q(f)$ for all $f \in \mathcal{S}_{\omega}\left(\mathbb{R}^{d}\right)$. When taking the limit, we have

$$
p\left(A_{\chi}(f)\right) \leq C q(f), \quad f \in \mathcal{S}_{\omega}\left(\mathbb{R}^{d}\right),
$$

which yields that $A_{\chi}: \mathcal{S}_{\omega}\left(\mathbb{R}^{d}\right) \rightarrow \mathcal{S}_{\omega}\left(\mathbb{R}^{d}\right)$ is continuous.

We show formula (1.5). Indeed, by Lemma 1.10 we have, for each $n \in \mathbb{N}$, that for all $\lambda>0$ there exists $C_{\lambda}>0$ such that

$\left|\int e^{i(x-y) \cdot \xi} a(x, y, \xi) f(y) \chi\left(\frac{1}{n}(x, \xi)\right) d y\right| \leq C_{\lambda} e^{-\lambda \omega(\xi)} e^{\max \left\{m, m L^{2}\right\} \omega(x)} \sup _{\eta \in \mathbb{R}^{2 d}}|\chi(\eta)|$,

which is integrable in $\xi$. Moreover,

$$
\int e^{i(x-y) \cdot \xi} a(x, y, \xi) f(y) \chi\left(\frac{1}{n}(x, \xi)\right) d y \longrightarrow \int e^{i(x-y) \cdot \xi} a(x, y, \xi) f(y) d y
$$

pointwise on $x, \xi \in \mathbb{R}^{d}$ as $n$ goes to infinity. An application of Lebesgue theorem gives the conclusion.

Definition 1.16. The operator $A$ in (1.5) is called global $\omega$-pseudodifferential operator associated to the amplitude $a(x, y, \xi)$.

When we consider a global symbol $a \in \mathrm{GS}_{\rho}^{m, \omega}$, its corresponding global $\omega$ pseudodifferential operator is given by

$$
a(x, D) f(x):=\int_{\mathbb{R}^{d}} e^{i x \cdot \xi} a(x, \xi) \widehat{f}(\xi) d \xi, \quad f \in \mathcal{S}_{\omega}\left(\mathbb{R}^{d}\right), x \in \mathbb{R}^{d} .
$$


Remark 1.17. In the hypothesis of Proposition 1.9 we could have also used $\chi(\xi)$ in $\mathcal{S}_{\omega}\left(\mathbb{R}^{d}\right)$ instead of $\chi(x, \xi) \in \mathcal{S}_{\omega}\left(\mathbb{R}^{2 d}\right)$. Also, Theorem 1.15 holds true if we consider $\chi(\xi)$ in $\mathcal{S}_{\omega}\left(\mathbb{R}^{d}\right)$ with $\chi(0)=1$ instead of $\chi(x, \xi) \in \mathcal{S}_{\omega}\left(\mathbb{R}^{2 d}\right)$. Both results follow in the same way.

The use of amplitudes permits to extend the operator to spaces of ultradistributions in an easy way (by duality). The following result is similar to [33, Theorem 2.5].

Proposition 1.18. The pseudodifferential operator $A: \mathcal{S}_{\omega}\left(\mathbb{R}^{d}\right) \rightarrow \mathcal{S}_{\omega}\left(\mathbb{R}^{d}\right)$ extends to a linear and continuous operator $\widetilde{A}: \mathcal{S}_{\omega}^{\prime}\left(\mathbb{R}^{d}\right) \rightarrow \mathcal{S}_{\omega}^{\prime}\left(\mathbb{R}^{d}\right)$.

Proof. Given the amplitude $a(x, y, \xi) \in \mathrm{GA}_{\rho}^{m, \omega}$, we denote

$$
b(x, y, \xi):=a(y, x,-\xi),
$$

also in $\mathrm{GA}_{\rho}^{m, \omega}$. We denote by $B$ its associated pseudodifferential operator. The transpose operator of $B$ defines a linear and continuous operator $\widetilde{A}:=$ $B^{t}: \mathcal{S}_{\omega}^{\prime}\left(\mathbb{R}^{d}\right) \rightarrow \mathcal{S}_{\omega}^{\prime}\left(\mathbb{R}^{d}\right)$ and we check that $\left.\widetilde{A}\right|_{\mathcal{S}_{\omega}\left(\mathbb{R}^{d}\right)}=A$. We denote

$$
\begin{aligned}
& \left(B_{\delta} \phi\right)(x)=\iint e^{i(x-y) \cdot \xi} b(x, y, \xi) \chi(\delta \xi) \phi(y) d y d \xi \\
& \left(A_{\delta} \psi\right)(x)=\iint e^{i(x-y) \cdot \xi} a(x, y, \xi) \chi(\delta \xi) \psi(y) d y d \xi
\end{aligned}
$$

for $\delta>0, x \in \mathbb{R}^{d}$, and $\chi$ is a function in $\mathcal{S}_{\omega}\left(\mathbb{R}^{d}\right)$ with $\chi(0)=1$. By Fubini's theorem we obtain (see [47, Teorema 1.2.5])

$$
\int \psi\left(B_{\delta} \phi\right)=\int\left(A_{\delta} \psi\right) \phi, \quad \psi, \phi \in \mathcal{S}_{\omega}\left(\mathbb{R}^{d}\right) .
$$

Proceeding as in the proof of Theorem 1.15 (see Remark 1.17), we obtain, by Lebesgue theorem,

$$
\int \psi(B \phi)=\int(A \psi) \phi, \quad \psi, \phi \in \mathcal{S}_{\omega}\left(\mathbb{R}^{d}\right) .
$$

From the proof of Proposition 1.18 we obtain that, given a pseudodifferential operator $A: \mathcal{S}_{\omega}\left(\mathbb{R}^{d}\right) \rightarrow \mathcal{S}_{\omega}\left(\mathbb{R}^{d}\right)$ with amplitude $a(x, y, \xi)$, the transpose operator restricted to $\mathcal{S}_{\omega}\left(\mathbb{R}^{d}\right),\left.A^{t}\right|_{\mathcal{S}_{\omega}\left(\mathbb{R}^{d}\right)}: \mathcal{S}_{\omega}\left(\mathbb{R}^{d}\right) \rightarrow \mathcal{S}_{\omega}\left(\mathbb{R}^{d}\right)$, is still a pseudodifferential operator, with amplitude $a(y, x,-\xi)$.

The following result clarifies the role operators with kernel in $\mathcal{S}_{\omega}\left(\mathbb{R}^{2 d}\right)$ play. 
Proposition 1.19. Let $A: \mathcal{S}_{\omega}\left(\mathbb{R}^{d}\right) \rightarrow \mathcal{S}_{\omega}\left(\mathbb{R}^{d}\right)$ be a pseudodifferential operator. The following assertions are equivalent:

(1) A has a linear and continuous extension $\widetilde{A}: \mathcal{S}_{\omega}^{\prime}\left(\mathbb{R}^{d}\right) \rightarrow \mathcal{S}_{\omega}\left(\mathbb{R}^{d}\right)$.

(2) There exists $K \in \mathcal{S}_{\omega}\left(\mathbb{R}^{2 d}\right)$ such that

$$
(A \varphi)(x)=\int_{\mathbb{R}^{d}} K(x, y) \varphi(y) d y, \quad \varphi \in \mathcal{S}_{\omega}\left(\mathbb{R}^{d}\right) .
$$

Proof. (1) $\Rightarrow(2)$ We define $K(x, y):=\widetilde{A}\left(\delta_{y}\right)(x)$ for $x, y \in \mathbb{R}^{d}$, where $\delta_{y}$ : $\mathcal{S}_{\omega}\left(\mathbb{R}^{d}\right) \rightarrow \mathbb{C}, \delta_{y}(f)=f(y)$ is the evaluation map (clearly, $\delta_{y} \in \mathcal{E}_{(\omega)}^{\prime}\left(\mathbb{R}^{d}\right) \subseteq$ $\left.\mathcal{S}_{\omega}^{\prime}\left(\mathbb{R}^{d}\right)\right)$. First, for fixed $y_{0} \in \mathbb{R}^{d}$ and $e_{i} \in \mathbb{R}^{d}$, an element of the canonical basis for some $1 \leq i \leq d$, we check

$$
-\partial_{y_{i}} \delta_{y_{0}}=\mathcal{S}_{\omega}^{\prime}\left(\mathbb{R}^{d}\right)-\lim _{t \rightarrow 0} \frac{\delta_{y_{0}+t e_{i}}-\delta_{y_{0}}}{t} .
$$

Indeed, for $f \in \mathcal{S}_{\omega}\left(\mathbb{R}^{d}\right)$,

$$
\lim _{t \rightarrow 0}\left\langle f, \frac{\delta_{y_{0}+t e_{i}}-\delta_{y_{0}}}{t}\right\rangle=\lim _{t \rightarrow 0} \frac{1}{t}\left(f\left(y_{0}+t e_{i}\right)-f\left(y_{0}\right)\right)=\partial_{y_{i}} f\left(y_{0}\right)=\left\langle f,-\partial_{y_{i}} \delta_{y_{0}}\right\rangle .
$$

From the continuity of $\widetilde{A}: \mathcal{S}_{\omega}^{\prime}\left(\mathbb{R}^{d}\right) \rightarrow \mathcal{S}_{\omega}\left(\mathbb{R}^{d}\right)$, we have

$$
\lim _{t \rightarrow 0}\left\langle\mu, \widetilde{A}\left(\frac{\delta_{y_{0}+t e_{i}}-\delta_{y_{0}}}{t}\right)\right\rangle=\left\langle\mu, \widetilde{A}\left(-\partial_{y_{i}} \delta_{y_{0}}\right)\right\rangle, \quad \mu \in \mathcal{S}_{\omega}^{\prime}\left(\mathbb{R}^{d}\right) .
$$

In particular, for $\mu=\delta_{x_{0}}, x_{0} \in \mathbb{R}^{d}$, we obtain that

$$
\begin{aligned}
\left\langle\delta_{x_{0}}, \widetilde{A}\left(\frac{\delta_{y_{0}+t e_{i}}-\delta_{y_{0}}}{t}\right)\right\rangle & =\frac{1}{t}\left(\widetilde{A}\left(\delta_{y_{0}+t e_{i}}\right)\left(x_{0}\right)-\widetilde{A}\left(\delta_{y_{0}}\right)\left(x_{0}\right)\right) \\
& =\frac{1}{t}\left(K\left(x_{0}, y_{0}+t e_{i}\right)-K\left(x_{0}, y_{0}\right)\right)
\end{aligned}
$$

tends to

$$
\left\langle\delta_{x_{0}}, \widetilde{A}\left(-\partial_{y_{i}} \delta_{y_{0}}\right)\right\rangle=\widetilde{A}\left(-\partial_{y_{i}} \delta_{y_{0}}\right)\left(x_{0}\right)
$$

when $t \rightarrow 0$. Given $\alpha \in \mathbb{N}_{0}^{d}$ we easily have

$$
\partial_{y}^{\alpha} K\left(x_{0}, y_{0}\right)=\widetilde{A}\left((-1)^{|\alpha|} \partial_{y}^{\alpha} \delta_{y_{0}}\right)\left(x_{0}\right),
$$

by proceeding by induction on $|\alpha|$. Hence, as $\widetilde{A}\left((-1)^{|\alpha|} \partial_{y}^{\alpha} \delta_{y_{0}}\right)(\cdot) \in \mathcal{S}_{\omega}\left(\mathbb{R}^{d}\right)$, we get that $\partial_{x}^{\beta} \partial_{y}^{\alpha} K(x, y)$ exists (and is continuous) for all $\alpha, \beta \in \mathbb{N}_{0}^{d}$, thus $K \in C^{\infty}\left(\mathbb{R}^{2 d}\right)$. 
To show that $K \in \mathcal{S}_{\omega}\left(\mathbb{R}^{2 d}\right)$ we denote, for $k \in \mathbb{N}$,

$$
\mathcal{B}:=\left\{(-1)^{|\alpha|} \partial_{y}^{\alpha} \delta_{y} e^{-k \varphi^{*}\left(\frac{|\alpha|}{k}\right)} e^{k L \omega(y)}: \alpha \in \mathbb{N}_{0}^{d}, y \in \mathbb{R}^{d}\right\}
$$

which is (weakly) bounded in $\mathcal{S}_{\omega}^{\prime}\left(\mathbb{R}^{d}\right)$. Indeed, for $f \in \mathcal{S}_{\omega}\left(\mathbb{R}^{d}\right)$,

$$
\begin{aligned}
& \sup _{\alpha \in \mathbb{N}_{0}^{d}} \sup _{y \in \mathbb{R}^{d}}\left|\left\langle f,(-1)^{|\alpha|} \partial_{y}^{\alpha} \delta_{y} e^{-k \varphi^{*}\left(\frac{|\alpha|}{k}\right)} e^{k L \omega(y)}\right\rangle\right| \\
& =\sup _{\alpha \in \mathbb{N}_{0}^{d}} \sup _{y \in \mathbb{R}^{d}}\left|D^{\alpha} f(y)\right| e^{-k \varphi^{*}\left(\frac{|\alpha|}{k}\right)} e^{k L \omega(y)}<+\infty .
\end{aligned}
$$

Hence $\mathcal{B}$ is bounded in $\mathcal{S}_{\omega}\left(\mathbb{R}^{d}\right)$ (as $\mathcal{S}_{\omega}\left(\mathbb{R}^{d}\right)$ is barrelled). Then, $\widetilde{A}(\mathcal{B})$ is bounded in $\mathcal{S}_{\omega}\left(\mathbb{R}^{d}\right)$ since $\widetilde{A}$ is continuous. Therefore, for the same $k$ as before, we have

$$
\sup _{\alpha, \beta \in \mathbb{N}_{0}^{d}} \sup _{x, y \in \mathbb{R}^{d}}\left|D_{x}^{\beta} \widetilde{A}\left((-1)^{|\alpha|} \partial_{y}^{\alpha} \delta_{y}\right)(x)\right| e^{-k \varphi^{*}\left(\frac{|\alpha|}{k}\right)} e^{k L \omega(y)} e^{-k \varphi^{*}\left(\frac{|\beta|}{k}\right)} e^{k L \omega(x)}<+\infty .
$$

Thus, by (0.3) and (0.11), we deduce

$$
\sup _{\alpha, \beta \in \mathbb{N}_{0}^{d}} \sup _{x, y \in \mathbb{R}^{d}}\left|D_{x}^{\beta} D_{y}^{\alpha} K(x, y)\right| e^{-k \varphi^{*}\left(\frac{|\alpha+\beta|}{k}\right)} e^{k \omega(x, y)}<+\infty .
$$

This shows $K(x, y) \in \mathcal{S}_{\omega}\left(\mathbb{R}^{2 d}\right)$ since $k \in \mathbb{N}$ is arbitrary.

We finally check the formula in (2). We write $\mu:=\int \varphi(y) \delta_{y} d y \in \mathcal{S}_{\omega}^{\prime}\left(\mathbb{R}^{d}\right)$. For any $f \in \mathcal{S}_{\omega}\left(\mathbb{R}^{d}\right)$ we have $\langle\mu, f\rangle=\left\langle\int \varphi\left(\widetilde{A}^{y} \delta_{y} d y, f\right\rangle=\int \varphi(y) f(y) d y\right.$, which shows $\mu=\varphi$. Then, by the assumptions on $\widetilde{A}$, we obtain

$$
\begin{aligned}
(A \varphi)(x) & =\left\langle\delta_{x}, \widetilde{A}\left(\int \varphi(y) \delta_{y} d y\right)\right\rangle \\
& =\left\langle\delta_{x}, \int \varphi(y) \widetilde{A}\left(\delta_{y}\right) d y\right\rangle=\int \widetilde{A}\left(\delta_{y}\right)(x) \varphi(y) d y=\int K(x, y) \varphi(y) d y
\end{aligned}
$$

for all $x \in \mathbb{R}^{d}$.

$(2) \Rightarrow(1)$ By Proposition 1.18, $A$ admits a linear and continuous extension $\widetilde{A}: \mathcal{S}_{\omega}^{\prime}\left(\mathbb{R}^{d}\right) \rightarrow \mathcal{S}_{\omega}^{\prime}\left(\mathbb{R}^{d}\right)$. Since $\mathcal{S}_{\omega}\left(\mathbb{R}^{d}\right)$ is dense in $\mathcal{S}_{\omega}^{\prime}\left(\mathbb{R}^{d}\right)$, for $u \in \mathcal{S}_{\omega}^{\prime}\left(\mathbb{R}^{d}\right)$ there exists $\left\{u_{n}\right\}_{n} \subseteq \mathcal{S}_{\omega}\left(\mathbb{R}^{d}\right)$ that converges to $u$ (in the topology of $\mathcal{S}_{\omega}^{\prime}\left(\mathbb{R}^{d}\right)$ ).

We claim that $\left\{\widetilde{A}\left(u_{n}\right)\right\}_{n}$ is a Cauchy sequence in $\mathcal{S}_{\omega}\left(\mathbb{R}^{d}\right)$. First, we observe that, for all $k>0$,

$$
\mathcal{B}:=\left\{\partial_{x}^{\alpha} K(x, \cdot) e^{-k \varphi^{*}\left(\frac{|\alpha|}{k}\right)} e^{k \omega(x)}: \alpha \in \mathbb{N}_{0}^{d}, x \in \mathbb{R}^{d}\right\}
$$


is a bounded set in $\mathcal{S}_{\omega}\left(\mathbb{R}^{d}\right)$. Indeed, by $(0.11)$ we have, for all $k>0$,

$$
\begin{gathered}
\sup _{\alpha, \beta \in \mathbb{N}_{0}^{d}} \sup _{x, y \in \mathbb{R}^{d}}\left|\partial_{y}^{\beta} \partial_{x}^{\alpha} K(x, y)\right| e^{-k \varphi^{*}\left(\frac{|\alpha|}{k}\right)} e^{k \omega(x)} e^{-k \varphi^{*}\left(\frac{|\beta|}{k}\right)} e^{k \omega(y)} \\
\leq \sup _{\alpha, \beta \in \mathbb{N}_{0}^{d}} \sup _{x, y \in \mathbb{R}^{d}}\left|\partial_{y}^{\beta} \partial_{x}^{\alpha} K(x, y)\right| e^{-2 k \varphi^{*}\left(\frac{|\alpha+\beta|}{2 k}\right)} e^{2 k \omega(x, y)},
\end{gathered}
$$

which is finite since $K \in \mathcal{S}_{\omega}\left(\mathbb{R}^{2 d}\right)$. Then, the polar set $\mathcal{B}^{\circ}$ is a 0 -neighbourhood in $\mathcal{S}_{\omega}^{\prime}\left(\mathbb{R}^{d}\right)$. Since $\left\{u_{n}\right\}_{n}$ is a Cauchy sequence in $\mathcal{S}_{\omega}^{\prime}\left(\mathbb{R}^{d}\right)$, given $\varepsilon>0$ and $k>0$ there exists $n_{0} \in \mathbb{N}$ such that if $n, l \geq n_{0}$, then $u_{n}-u_{l} \in \varepsilon \mathcal{B}^{\circ}$; that is,

$$
\left|\left\langle\left(u_{n}-u_{l}\right)(\cdot), \partial_{x}^{\alpha} K(x, \cdot)\right\rangle\right| e^{-k \varphi^{*}\left(\frac{|\alpha|}{k}\right)} e^{k \omega(x)} \leq \varepsilon .
$$

On the other hand, we differentiate under the integral sign and we get

$$
\partial_{x}^{\alpha}\left(\widetilde{A} u_{n}-\widetilde{A} u_{l}\right)(x)=\int\left(u_{n}-u_{l}\right)(y) \partial_{x}^{\alpha} K(x, y) d y
$$

Thus,

$$
\begin{aligned}
& \sup _{\alpha \in \mathbb{N}_{0}^{d}} \sup _{x \in \mathbb{R}^{d}}\left|\partial_{x}^{\alpha}\left(\widetilde{A} u_{n}-\widetilde{A} u_{l}\right)(x)\right| e^{-k \varphi^{*}\left(\frac{|\alpha|}{k}\right)} e^{k \omega(x)} \\
& \quad=\sup _{\alpha \in \mathbb{N}_{0}^{d}} \sup _{x \in \mathbb{R}^{d}}\left|\int\left(u_{n}-u_{l}\right)(y) \partial_{x}^{\alpha} K(x, y) d y\right| e^{-k \varphi^{*}\left(\frac{|\alpha|}{k}\right)} e^{k \omega(x)} \leq \varepsilon,
\end{aligned}
$$

and the claim is shown.

Since $\mathcal{S}_{\omega}\left(\mathbb{R}^{d}\right)$ is Fréchet, the sequence $\left\{\widetilde{A}\left(u_{n}\right)\right\}_{n}$ converges to some $f \in \mathcal{S}_{\omega}\left(\mathbb{R}^{d}\right)$. By the uniqueness of the limit, $\widetilde{A}(u)=f \in \mathcal{S}_{\omega}\left(\mathbb{R}^{d}\right)$, as $u_{n} \rightarrow u$ in $\mathcal{S}_{\omega}^{\prime}\left(\mathbb{R}^{d}\right)$. This shows that $\widetilde{A}\left(\mathcal{S}_{\omega}^{\prime}\left(\mathbb{R}^{d}\right)\right) \subseteq \mathcal{S}_{\omega}\left(\mathbb{R}^{d}\right)$. An application of the closed graph theorem shows that $\widetilde{A}: \mathcal{S}_{\omega}^{\prime}\left(\mathbb{R}^{d}\right) \rightarrow \mathcal{S}_{\omega}\left(\mathbb{R}^{d}\right)$ is continuous.

Definition 1.20. A pseudodifferential operator $A: \mathcal{S}_{\omega}\left(\mathbb{R}^{d}\right) \rightarrow \mathcal{S}_{\omega}\left(\mathbb{R}^{d}\right)$ satisfying (1) or (2) of Proposition 1.19 is called $\omega$-regularizing.

Example 1.21. (a) Particular cases of weight functions give already known definitions of symbol classes and pseudodifferential operators as in [33, Example 2.11]. For example, when $\omega(t)=\log (1+t)$, for which $\mathcal{S}_{\omega}\left(\mathbb{R}^{d}\right)=\mathcal{S}\left(\mathbb{R}^{d}\right)$, it is known (see for instance [47, Ejemplo 1.3.1]) that $\varphi_{\omega}^{*}(t)$ is either 0 (for $t \leq 1$ ) or $+\infty$ (for $t>1$ ). In this case, we have that $a \in \mathrm{GA}_{\rho}^{m, \omega}$ if and only if for all $\alpha, \beta, \gamma \in \mathbb{N}_{0}^{d}$ there exists $C>0$ such that

$$
\left|D_{x}^{\alpha} D_{y}^{\gamma} D_{\xi}^{\beta} a(x, y, \xi)\right| \leq C\langle x-y\rangle^{\rho|\alpha+\gamma+\beta|}\langle(x, y, \xi)\rangle^{m-\rho|\alpha+\gamma+\beta|}, \quad x, y, \xi \in \mathbb{R}^{d} .
$$


This characterization coincides with [64, Definition 23.3] for $m^{\prime}=0$ (see [33, Example 2.11(1)] for a symbol in the sense of Grigis and Sjöstrand [37]).

In the same way, if we consider a Gevrey weight $\left(\omega(t)=t^{p}\right.$ for some $\left.0<p<1\right)$, then for all $n \in \mathbb{N}$ there exist $A_{n}, B_{n}>0$ such that (see [47, Ejemplo 1.3.2])

$$
A_{n}(\alpha !)^{1 / p}\left(\frac{1}{n p}\right)^{|\alpha| / p} \leq e^{n \varphi_{\omega}^{*}\left(\frac{|\alpha|}{n}\right)} \leq B_{n}(\alpha !)^{1 / p}\left(\frac{2 d}{n p}\right)^{|\alpha| / p}, \quad \alpha \in \mathbb{N}_{0}^{d} .
$$

Therefore, if $a \in \mathrm{GA}_{\rho}^{m, \omega}$, for all $n \in \mathbb{N}$ there exists $C_{n^{\prime}}>0$, where we denote $n^{\prime}=\frac{2 d}{p n^{1 / \rho}}>0$, such that

$$
\begin{aligned}
& \left|D_{x}^{\alpha} D_{y}^{\gamma} D_{\xi}^{\beta} a(x, y, \xi)\right| \\
& \quad \leq C_{n^{\prime}}\left(\frac{\langle x-y\rangle}{\langle(x, y, \xi)\rangle}\right)^{\rho|\alpha+\gamma+\beta|} e^{n^{\prime} \rho \varphi^{*}\left(\frac{|\alpha+\gamma+\beta|}{n^{\prime}}\right)} e^{m \omega(x, y, \xi)} \\
& \quad \leq C_{n^{\prime}}\left(\frac{\langle x-y\rangle}{\langle(x, y, \xi)\rangle}\right)^{\rho|\alpha+\gamma+\beta|} B_{n^{\prime}}^{\rho}(\alpha ! \gamma ! \beta !)^{\rho / p} n^{|\alpha+\gamma+\beta| / p} e^{m|(x, y, \xi)|^{p}}
\end{aligned}
$$

for all $\alpha, \gamma, \beta \in \mathbb{N}_{0}^{d}, x, y, \xi \in \mathbb{R}^{d}$. Conversely, given $n \in \mathbb{N}$ take $n^{\prime}=(n p)^{-\rho}>0$. Then, there exist $C_{n^{\prime}}^{\prime}, C_{n^{\prime}}^{\prime \prime}>0$ so that

$$
\begin{aligned}
\mid D_{x}^{\alpha} & D_{y}^{\gamma} D_{\xi}^{\beta} a(x, y, \xi) \mid \\
& \leq C_{n^{\prime}}^{\prime}\left(\frac{\langle x-y\rangle}{\langle(x, y, \xi)\rangle}\right)^{\rho|\alpha+\gamma+\beta|}(\alpha ! \gamma ! \beta !)^{\rho / p}\left(n^{\prime}\right)^{|\alpha+\gamma+\beta| / p} e^{m|(x, y, \xi)|^{p}} \\
& \leq C_{n^{\prime}}^{\prime \prime}\left(\frac{\langle x-y\rangle}{\langle(x, y, \xi)\rangle}\right)^{\rho|\alpha+\gamma+\beta|} e^{n \rho \varphi^{*}\left(\frac{|\alpha+\gamma+\beta|}{n}\right)} e^{m \omega(x, y, \xi)},
\end{aligned}
$$

for each $\alpha, \gamma, \beta \in \mathbb{N}_{0}^{d}, x, y, \xi \in \mathbb{R}^{d}$. Then, this definition of amplitude could be compared with Cappiello [23, Definition 2.1] (see also Rodino [60]), which is the corresponding definition for the Gevrey class (of Roumieu type).

(b) Given a weight function $\omega$, for $0<\rho \leq 1$, take another weight function $\sigma$ satisfying $\omega\left(t^{(1+\rho) / \rho}\right)=O(\sigma(t))$ as $t \rightarrow \infty$. We show that

$$
\mathcal{S}_{\sigma}\left(\mathbb{R}^{2 d}\right) \subseteq \bigcap_{m \in \mathbb{R}} \mathrm{GS}_{\rho}^{m, \omega} \subseteq \mathcal{S}_{\omega}\left(\mathbb{R}^{2 d}\right)
$$

Indeed, if $p(x, \xi) \in \mathcal{S}_{\sigma}\left(\mathbb{R}^{2 d}\right)$, then for all $\lambda, m>0$ there is $C_{\lambda, m}=C_{\lambda,(\lambda+m) L}>0$ with (we use (0.6), but we do not know if $\sigma(1)=0$ )

$$
\begin{aligned}
\left|D_{x}^{\alpha} D_{\xi}^{\beta} p(x, \xi)\right| & \leq C_{\lambda, m} e^{\lambda \varphi_{\sigma}^{*}\left(\frac{|\alpha+\beta|}{\lambda}\right)} e^{-(\lambda+m) L \sigma(x, \xi)} \\
& \leq C_{\lambda, m} e^{(\lambda+m) L(1+\sigma(1))} e^{\lambda \varphi_{\sigma}^{*}\left(\frac{|\alpha+\beta|}{\lambda}\right)} e^{-(\lambda+m) \sigma(\langle(x, \xi)\rangle)}
\end{aligned}
$$


for all $\alpha, \beta \in \mathbb{N}_{0}^{d}, x, \xi \in \mathbb{R}^{d}$. For all $\lambda>0$, formula (0.7) yields

$$
\begin{aligned}
e^{\lambda \varphi_{\sigma}^{*}\left(\frac{|\alpha+\beta|}{\lambda}\right)} e^{-(\lambda+m) \sigma(\langle(x, \xi)\rangle)} & \leq e^{\lambda(1+\rho) \varphi_{\sigma}^{*}\left(\frac{|\alpha+\beta|}{\lambda}\right)} e^{-\lambda \rho \varphi_{\sigma}^{*}\left(\frac{|\alpha+\beta|}{\lambda}\right)} e^{-(\lambda \rho+m) \sigma(\langle(x, \xi)\rangle)} \\
& \leq e^{\lambda(1+\rho) \varphi_{\sigma}^{*}\left(\frac{|\alpha+\beta|}{\lambda}\right)}\langle(x, \xi)\rangle^{-\rho|\alpha+\beta|} e^{-m \sigma(\langle(x, \xi)\rangle)} .
\end{aligned}
$$

By hypothesis there is $C>0$ such that $\omega(t) \leq C \sigma(t)+C \leq C \sigma(\langle t\rangle)+C$, for all $t \geq 0$. Moreover, from Lemma $0.10(2)$, we obtain

$$
\lambda(1+\rho) \varphi_{\sigma}^{*}\left(\frac{j}{\lambda}\right) \leq \lambda+\frac{\lambda}{C} \rho \varphi_{\omega}^{*}\left(\frac{j C}{\lambda}\right), \quad j \in \mathbb{N}_{0} .
$$

Thus, we have $p \in \mathrm{GS}_{\rho}^{-m / C, \omega}$. Due to the arbitrariness of $m>0$, we get the first inclusion. The second inclusion is immediate.

The weight functions $\omega(t)=\log ^{s}(1+t), t \geq 0$, with $s \geq 1$, satisfy $\omega\left(t^{(1+\rho) / \rho}\right)=$ $O(\omega(t))$ as $t \rightarrow \infty$. Hence, for $\omega(t)=\log ^{s}(1+t)$,

$$
\bigcap_{m \in \mathbb{R}} \mathrm{GS}_{\rho}^{m, \omega}=\mathcal{S}_{\omega}\left(\mathbb{R}^{2 d}\right)
$$

In fact, for all $t \geq 0$, we have

$$
\begin{aligned}
\omega\left(t^{(1+\rho) / \rho}\right) & =\log ^{s}\left(1+t^{(1+\rho) / \rho}\right) \leq \log ^{s}\left((1+t)^{(1+\rho) / \rho}\right) \\
& \leq\left(\frac{1+\rho}{\rho}\right)^{s} \log ^{s}(1+t)=\left(\frac{1+\rho}{\rho}\right)^{s} \omega(t) .
\end{aligned}
$$

So, the identity (1.22) follows from the previous arguments.

(c) We consider now an ultradifferential operator of $(\omega)$-class in the sense of Komatsu [49] of type

$$
G(x, D):=\sum_{\gamma \in \mathbb{N}_{0}^{d}} a_{\gamma}(x) D^{\gamma},
$$

where $a_{\gamma}$ is a $C^{\infty}\left(\mathbb{R}^{d}\right)$ function satisfying that there exist $0<\rho \leq 1$ and $n \in \mathbb{N}$ such that for all $\lambda>0$, there exists $C_{\lambda}>0$, with

$$
\sup _{x \in \mathbb{R}^{d}}\left|D^{\alpha} a_{\gamma}(x)\right| \leq C_{\lambda} e^{\lambda \rho \varphi^{*}\left(\frac{|\alpha|}{\lambda}\right)} e^{-n \varphi^{*}\left(\frac{|\alpha+\gamma|}{n}\right)}, \quad \alpha, \gamma \in \mathbb{N}_{0}^{d} .
$$

We want to show that $p(x, \xi):=(2 \pi)^{-d} \sum_{\gamma \in \mathbb{N}_{0}^{d}} a_{\gamma}(x) \xi^{\gamma}$ is a global symbol as in Definition 1.1. First, we need to check that $p(x, \xi)$ defines a series that 
converges uniformly in $x \in \mathbb{R}^{d}$ (and pointwise in $\xi \in \mathbb{R}^{d}$ ). To this, we fix $x \in \mathbb{R}^{d}$ and we have that there exists $C_{n}>0$ such that (by Lemma $0.1(7)$ )

$$
\begin{aligned}
|p(x, \xi)| & \leq \sum_{\gamma \in \mathbb{N}_{0}^{d}}\left|a_{\gamma}(x)\right||\xi|^{|\gamma|} \\
& \leq \sum_{\gamma \in \mathbb{N}_{0}^{d}} C_{n} e^{-n \varphi^{*}\left(\frac{|\gamma|}{n}\right)}|\xi|^{|\gamma|} \leq \sum_{k=0}^{\infty} C_{n} e^{-n \varphi^{*}\left(\frac{k}{n}\right)}|\xi|^{k} \sum_{|\gamma|=k} 1 \\
& \leq C_{n} \sum_{k=0}^{\infty} e^{-n \varphi^{*}\left(\frac{k}{n}\right)}|\xi|^{k} 2^{k+d-1}=2^{d-1} C_{n} \sum_{k=0}^{\infty} e^{-n \varphi^{*}\left(\frac{k}{n}\right)}(2|\xi|)^{k} .
\end{aligned}
$$

We use formula (0.7) to obtain

$$
|p(x, \xi)| \leq 2^{d-1} C_{n} \sum_{k=0}^{\infty}\left(\frac{1}{2}\right)^{k} e^{-n \varphi^{*}\left(\frac{k}{n}\right)}(4\langle\xi\rangle)^{k} \leq 2^{d-1} C_{n} e^{n \omega(4\langle\xi\rangle)} \sum_{k=0}^{\infty}\left(\frac{1}{2}\right)^{k}
$$

Now, for $\gamma \in \mathbb{N}_{0}^{d}$ we estimate the derivatives of $a_{\gamma}(x) \xi^{\gamma}$ for all $x, \xi \in \mathbb{R}^{d}$ as follows: there are $0<\rho \leq 1$ and $n \in \mathbb{N}$ such that for all $\lambda>0$ there is $C_{\lambda}>0$ such that, by (0.11), we have for all $\alpha, \beta \in \mathbb{N}_{0}^{d}, \beta \leq \gamma$,

$$
\begin{aligned}
& \left|D_{x}^{\alpha} D_{\xi}^{\beta}\left(a_{\gamma}(x) \xi^{\gamma}\right)\right| \leq C_{\lambda} e^{\lambda \rho \varphi^{*}\left(\frac{|\alpha|}{\lambda}\right)} e^{-n \varphi^{*}\left(\frac{|\alpha+\gamma|}{n}\right)} \frac{\gamma !}{(\gamma-\beta) !}|\xi|^{|\gamma-\beta|} \\
& \leq C_{\lambda} e^{\lambda \rho \varphi^{*}\left(\frac{|\alpha|}{\lambda}\right)} e^{-n \varphi^{*}\left(\frac{|\alpha|}{n}\right)} e^{-n \varphi^{*}\left(\frac{|\gamma|}{n}\right)} 2^{|\gamma|} \beta !\langle(x, \xi)\rangle^{|\gamma|-\rho|\beta|} .
\end{aligned}
$$

We use Lemma 0.9 (under the assumption $\omega(t)=o\left(t^{\rho}\right)$ as $t \rightarrow \infty$ ) to get that there exists $C_{\lambda}^{\prime}>0$ such that $\beta ! \leq C_{\lambda}^{\prime} e^{\lambda \rho \varphi^{*}\left(\frac{|\beta|}{\lambda}\right)}$. From Lemma 0.1 and $(0.7)$ we have

$$
\begin{aligned}
\left|D_{x}^{\alpha} D_{\xi}^{\beta}\left(a_{\gamma}(x) \xi^{\gamma}\right)\right| \leq & C_{\lambda} e^{\lambda \rho \varphi^{*}\left(\frac{|\alpha|}{\lambda}\right)} e^{-n \varphi^{*}\left(\frac{|\alpha|}{n}\right)} e^{-n \varphi^{*}\left(\frac{|\gamma|}{n}\right)} 2^{|\gamma|} \times \\
& \times C_{\lambda}^{\prime} e^{\lambda \rho \varphi^{*}\left(\frac{|\beta|}{\lambda}\right)}\langle(x, \xi)\rangle^{-\rho|\beta|} e^{n L^{2} \varphi^{*}\left(\frac{|\gamma|}{n L^{2}}\right)} e^{n L^{2} \omega(\langle(x, \xi)\rangle)} .
\end{aligned}
$$

We use (0.10) to get

$$
e^{-n \varphi^{*}\left(\frac{|\gamma|}{n}\right)} 2^{|\gamma|} e^{n L^{2} \varphi^{*}\left(\frac{|\gamma|}{n L^{2}}\right)} \leq\left(\frac{2}{e^{2}}\right)^{|\gamma|} e^{n L+n L^{2}} \leq e^{-|\gamma|} e^{n L+n L^{2}}
$$

Again, by (0.7) it follows that

$$
e^{-n \varphi^{*}\left(\frac{|\alpha|}{n}\right)} \leq\langle(x, \xi)\rangle^{-|\alpha|} e^{n \omega(\langle(x, \xi)\rangle)} \leq\langle(x, \xi)\rangle^{-\rho|\alpha|} e^{n \omega(\langle(x, \xi)\rangle)} .
$$


Hence, for $C_{\lambda}^{\prime \prime}=C_{\lambda} C_{\lambda}^{\prime} e^{n L+n L^{2}}>0$,

$$
\left|D_{x}^{\alpha} D_{\xi}^{\beta} p(x, \xi)\right| \leq C_{\lambda}^{\prime \prime} e^{\lambda \rho \varphi^{*}\left(\frac{|\alpha+\beta|}{\lambda}\right)}\langle(x, \xi)\rangle^{-\rho|\alpha+\beta|} e^{n\left(1+L^{2}\right) \omega(\langle(x, \xi)\rangle)} \sum_{\gamma \in \mathbb{N}_{0}^{d}} e^{-|\gamma|} .
$$

The series converges by Lemma 0.1(8). Then, by (0.6) we obtain, for $m=$ $n L\left(1+L^{2}\right)>0$, that $p \in \mathrm{GS}_{\rho}^{m, \omega}$.

We take $f \in \mathcal{S}_{\omega}\left(\mathbb{R}^{d}\right)$. Then, by formula (0.34) it follows that

$$
G(x, D) f(x)=\sum_{\gamma \in \mathbb{N}_{0}^{d}} a_{\gamma}(x) D^{\gamma} f(x)=(2 \pi)^{-d} \sum_{\gamma \in \mathbb{N}_{0}^{d}} a_{\gamma}(x) \int e^{i x \cdot \xi} \xi^{\gamma} \widehat{f}(\xi) d \xi .
$$

The latter series is convergent. Indeed, there exists $C_{n}>0$ such that by Lemma 0.1

$$
\begin{aligned}
\sum_{\gamma \in \mathbb{N}_{0}^{d}}\left|a_{\gamma}(x)\right| \int|\xi|^{|\gamma|}|\widehat{f}(\xi)| d \xi & \leq \sum_{k=0}^{\infty} C_{n} e^{-n \varphi^{*}\left(\frac{k}{n}\right)} \int|\xi|^{k}|\widehat{f}(\xi)| 2^{d+k-1} d \xi \\
& =2^{d-1} C_{n} \sum_{k=0}^{\infty}\left(\frac{1}{2}\right)^{k} e^{-n \varphi^{*}\left(\frac{k}{n}\right)} \int(4|\xi|)^{k}|\widehat{f}(\xi)| d \xi
\end{aligned}
$$

From $(0.7)$ and $(0.6)$, we obtain that $(4|\xi|)^{k} e^{-n \varphi^{*}\left(\frac{k}{n}\right)} \leq e^{n \omega(4\langle\xi\rangle)} \leq e^{m^{\prime} \omega(\xi)} e^{m^{\prime}}$, for some $m^{\prime}>0$. Thus,

$$
\sum_{\gamma \in \mathbb{N}_{0}^{d}}\left|a_{\gamma}(x)\right| \int|\xi|^{|\gamma|}|\widehat{f}(\xi)| d \xi \leq C_{n} e^{m^{\prime}}\left(\sum_{k=0}^{\infty}\left(\frac{1}{2}\right)^{k}\right) \int e^{m^{\prime} \omega(\xi)}|\widehat{f}(\xi)| d \xi,
$$

which shows that the series converges, since $f \in \mathcal{S}_{\omega}\left(\mathbb{R}^{d}\right)$. Therefore, by the Lebesgue theorem it follows that

$$
\begin{aligned}
G(x, D) f(x)=\int e^{i x \cdot \xi}\left((2 \pi)^{-d} \sum_{\gamma \in \mathbb{N}_{0}^{d}} a_{\gamma}(x) \xi^{\gamma}\right) \widehat{f}(\xi) d \xi & =\int e^{i x \cdot \xi} p(x, \xi) \widehat{f}(\xi) d \xi \\
& =P(x, D) f(x) .
\end{aligned}
$$

Notice that since the constant $n \in \mathbb{N}$ is fixed, the coefficients $a_{\gamma}$ satisfy a similar estimate as in (0.29) in Corollary 0.23 for the ultradifferential operator of $(\omega)$-class $G^{n}(D)$ with constant coefficients.

(d) As a consequence of (c), we can easily study some linear partial differential operators with variable coefficients which are examples of pseudodifferential 
operators. We consider

$$
P(x, D):=\sum_{|\gamma| \leq m} a_{\gamma}(x) D^{\gamma}
$$

for some $m \in \mathbb{N}$, where $a_{\gamma} \in C^{\infty}\left(\mathbb{R}^{d}\right)$, satisfying that there exist $0<\rho \leq 1$ and $n \in \mathbb{N}$ such that for all $\lambda>0$ there is $C_{\lambda}>0$ with

$$
\sup _{x \in \mathbb{R}^{d}}\left|D^{\alpha} a_{\gamma}(x)\right| \leq C_{\lambda} e^{\lambda \rho \varphi^{*}\left(\frac{|\alpha|}{\lambda}\right)} e^{-n \varphi^{*}\left(\frac{|\alpha|}{n}\right)}, \quad \alpha \in \mathbb{N}_{0}^{d} .
$$

Therefore, if $p(x, \xi):=(2 \pi)^{-d} \sum_{|\gamma| \leq m} a_{\gamma}(x) \xi^{\gamma}$, then by (c) we have that $p \in$ $\mathrm{GS}_{\rho}^{m^{\prime}, \omega}$ for $m^{\prime}=n L\left(1+L^{2}\right)>0$.

On the other hand, for a linear partial differential operator with polynomial coefficients, we have that

$$
p(x, \xi):=\sum_{|\eta| \leq n,|\gamma| \leq m} c_{\eta, \gamma} x^{\eta} \xi^{\gamma},
$$

where $c_{\eta, \gamma} \in \mathbb{C}$, is a global symbol of finite order in $\mathrm{S}_{\rho}^{n+m, \omega}$ in the sense of Definition 1.2, where $\omega(t)=o\left(t^{\rho}\right)$ as $t \rightarrow \infty$. In fact, there exists $C>0$ such that

$$
\left|D_{x}^{\alpha} D_{\xi}^{\beta} p(x, \xi)\right| \leq C \sum_{|\eta| \leq n,|\gamma| \leq m} \frac{\eta !}{(\eta-\alpha) !}|x|^{|\eta-\alpha|} \frac{\gamma !}{(\gamma-\beta) !}|\xi|^{|\gamma-\beta|}
$$

for all $\alpha \leq \eta, \beta \leq \gamma$. From Lemma 0.9 we have, since $\omega(t)=o\left(t^{\rho}\right)$ as $t \rightarrow \infty$,

$$
\frac{\eta !}{(\eta-\alpha) !} \frac{\gamma !}{(\gamma-\beta) !} \leq 2^{|\eta+\gamma|} \alpha ! \beta ! \leq 2^{n+m} e^{\lambda \rho \varphi^{*}\left(\frac{|\alpha+\beta|}{\lambda}\right)} .
$$

We have

$$
|x|^{|\eta-\alpha|}|\xi|^{|\gamma-\beta|} \leq\langle(x, \xi)\rangle^{|\eta+\gamma|-|\alpha+\beta|} \leq\langle(x, \xi)\rangle^{n+m-\rho|\alpha+\beta|} .
$$

Hence, for all $\lambda>0$ there exists $C_{\lambda, n, m}>0$ such that

$$
\left|D_{x}^{\alpha} D_{\xi}^{\beta} p(x, \xi)\right| \leq C_{\lambda, n, m}\langle(x, \xi)\rangle^{-\rho|\alpha+\beta|} e^{\lambda \rho \varphi^{*}\left(\frac{|\alpha+\beta|}{\lambda}\right)}\langle(x, \xi)\rangle^{n+m},
$$

for all $\alpha, \beta \in \mathbb{N}_{0}^{d}, x, \xi \in \mathbb{R}^{d}$. This shows that $p \in \mathrm{S}_{\rho}^{n+m, \omega}$, and also, by (0.7) we have $p \in \mathrm{GS}_{\rho}^{n+m, \omega}$. 
(e) An ultradifferential operator of $(\omega)$-class with constant coefficients is a global symbol if $\omega(t)=o\left(t^{\rho}\right), t \rightarrow \infty$, for some $0<\rho \leq 1$. In fact, let $G$ be an entire function in $\mathbb{C}^{d}$ such that $\log |G(z)|=O(\omega(z))$ as $|z| \rightarrow \infty$ (see $\left(i^{\prime}\right)$ of Theorem 0.18 ). We show that $G$, restricted to $\mathbb{R}^{2 d}$, is a global symbol in $\mathrm{GS}_{\rho}^{m, \omega}$ for some $m>0$.

Indeed, for $x, \xi \in \mathbb{R}^{d}$ we consider the polydisk $P((x, \xi), r)$ whose polyradius is $r:=\left(\langle(x, \xi)\rangle^{\rho}, \ldots,\langle(x, \xi)\rangle^{\rho}\right)$. By Proposition 0.11,

$$
\left|D_{x}^{\alpha} D_{\xi}^{\beta} G(x, \xi)\right| \leq \frac{\alpha ! \beta !}{r^{\alpha+\beta}} \sup _{(y, t) \in \partial P((x, \xi), r)}\{|G(y, t)|\}, \quad \alpha, \beta \in \mathbb{N}_{0}^{d}, x, \xi \in \mathbb{R}^{d} .
$$

By assumption there exists $C>0$ such that $|G(z)| \leq C e^{C \omega(z)}, z \in \mathbb{C}^{d}$. We see that if $(y, t) \in \partial P((x, \xi), r)$, then $|(y, t)-(x, \xi)|=\sqrt{d}\langle(x, \xi)\rangle^{\rho}$, so $|(y, t)| \leq$ $|(y, t)-(x, \xi)|+|(x, \xi)| \leq(1+\sqrt{d})\langle(x, \xi)\rangle$. As $r^{\alpha+\beta}=\langle(x, \xi)\rangle^{\rho|\alpha+\beta|}$, we have

$$
\left|D_{x}^{\alpha} D_{\xi}^{\beta} G(x, \xi)\right| \leq \alpha ! \beta !\langle(x, \xi)\rangle^{-\rho|\alpha+\beta|} C e^{C \omega((1+\sqrt{d})\langle(x, \xi)\rangle)},
$$

for all $\alpha, \beta \in \mathbb{N}_{0}^{d}, x, \xi \in \mathbb{R}^{d}$. From property $(\alpha)$ of $\omega$, there is $m>0$ satisfying

$$
C \omega((1+\sqrt{d})\langle(x, \xi)\rangle) \leq m \omega(x, \xi)+m .
$$

Finally, from Lemma 0.9 , for all $\lambda>0$ there exists $C_{\lambda}>0$ so that

$$
\alpha ! \beta ! \leq C_{\lambda} e^{\lambda \rho \varphi^{*}\left(\frac{|\alpha+\beta|}{\lambda}\right)},
$$

and hence

$$
\left|D_{x}^{\alpha} D_{\xi}^{\beta} G(x, \xi)\right| \leq C C_{\lambda} e^{m}\langle(x, \xi)\rangle^{-\rho|\alpha+\beta|} e^{\lambda \rho \varphi^{*}\left(\frac{|\alpha+\beta|}{\lambda}\right)} e^{m \omega(x, \xi)},
$$

for all $\alpha, \beta \in \mathbb{N}_{0}^{d}, x, \xi \in \mathbb{R}^{d}$, which shows $G \in \mathrm{GS}_{\rho}^{m, \omega}$. 


\section{Chapter 2}

\section{Quantizations for pseudodifferential operators}

In the present chapter we deal with the change of quantization in the class of global pseudodifferential operators introduced in Chapter 1. The symbols are of infinite order with exponential growth in all the variables (see Definition 1.1), in contrast to the approach of [33, 65], who treat pseudodifferential operators of infinite order in the local sense and infinite order only in the last variable, for classes of ultradifferentiable functions of Beurling type in the sense of [20] and for Gevrey classes. In [33], the composition of two operators is given in terms of a suitable symbolic calculus.

As we mention at the beginning, one of the main goals of this chapter is to extend the results in Chapter 1 by adapting them for a valid change of quantization for these symbols (see Definition 2.25). Namely, we follow the ideas for the change of quantization within the framework of global symbol classes of Shubin [64, §23]. In [58] it is considered the change of quantization and its corresponding symbolic calculus for classes in the sense of Komatsu [49], also in the Roumieu setting. However, our classes of symbols (and amplitudes) might not coincide with the ones defined in [58] even only in the Beurling setting, as mentioned in the introduction of Chapter 1. 
We develop the symbolic calculus and we state some previous results needed to compose two pseudodifferential operators. In this setting, to study the composition of two pseudodifferential operators, we need to show first the good behaviour (in terms of its estimates) of the kernel of a pseudodifferential operator outside a strip around the diagonal in Theorem 2.20. Thus, we improve [55, Theorem 6.3.3] and [58, Proposition 5], where similar estimates for Gevrey classes or classes of ultradifferentiable functions in the sense of Komatsu [49] are obtained only in the complement of a conical neighbourhood of the diagonal. This investigation leads to the construction of parametrices for pseudodifferential operators in Chapter 3.

The results of this chapter are contained in $[4,6]$.

\section{$2.1 \quad$ Symbolic calculus}

Definition 2.1. We define $\mathrm{FGS}_{\rho}^{m, \omega}$ as the set of all formal sums $\sum_{j \in \mathbb{N}_{0}} a_{j}(x, \xi)$ such that $a_{j}(x, \xi) \in C^{\infty}\left(\mathbb{R}^{2 d}\right)$ and there is $R \geq 1$ such that for every $n \in \mathbb{N}$ there exists $C_{n}>0$ with

$$
\left|D_{x}^{\alpha} D_{\xi}^{\beta} a_{j}(x, \xi)\right| \leq C_{n}\langle(x, \xi)\rangle^{-\rho(|\alpha+\beta|+j)} e^{n \rho \varphi^{*}\left(\frac{|\alpha+\beta|+j}{n}\right)} e^{m \omega(x, \xi)},
$$

for each $j \in \mathbb{N}_{0}, \alpha, \beta \in \mathbb{N}_{0}^{d}$ and $\log \left(\frac{\langle(x, \xi)\rangle}{R}\right) \geq \frac{n}{j} \varphi^{*}\left(\frac{j}{n}\right)$.

In Definition 2.1 we assume that $a_{0}(x, \xi)$ satisfies the estimate (2.1) for all $\log \left(\frac{\langle(x, \xi)\rangle}{R}\right) \geq 0$, i.e. for $\langle(x, \xi)\rangle \geq R$.

Let $a$ be a global symbol in $\mathrm{GS}_{\rho}^{m, \omega}$. The formal series $\sum_{j \in \mathbb{N}_{0}} a_{j}$ defined by $a_{0}:=a, a_{j}=0$ for $j \in \mathbb{N}$, belongs to $\mathrm{FGS}_{\rho}^{m, \omega}$; as a result, we may regard a global symbol as this formal sum $\sum_{j \in \mathbb{N}_{0}} a_{j}$.

Definition 2.2. Two formal sums $\sum a_{j}$ and $\sum b_{j}$ in $\mathrm{FGS}_{\rho}^{m, \omega}$ are called equivalent, denoted by $\sum a_{j} \sim \sum b_{j}$, if there is $R \geq 1$ such that for every $n \in \mathbb{N}$ there exist $C_{n}>0$ and $N_{n} \in \mathbb{N}$ with

$$
\left|D_{x}^{\alpha} D_{\xi}^{\beta} \sum_{j<N}\left(a_{j}-b_{j}\right)\right| \leq C_{n}\langle(x, \xi)\rangle^{-\rho(|\alpha+\beta|+N)} e^{n \rho \varphi^{*}\left(\frac{|\alpha+\beta|+N}{n}\right)} e^{m \omega(x, \xi)},
$$

for all $N \geq N_{n}, \alpha, \beta \in \mathbb{N}_{0}^{d}$, and $\log \left(\frac{\langle(x, \xi)\rangle}{R}\right) \geq \frac{n}{N} \varphi^{*}\left(\frac{N}{n}\right)$.

We understand that a global symbol $a \in \mathrm{GS}_{\rho}^{m, \omega}$ regarded as a formal sum satisfies $a \sim 0$ if there exists $R \geq 1$ such that for all $n \in \mathbb{N}$ there are $C_{n}>0$ 
and $N_{n} \in \mathbb{N}$ so that $\left|D_{x}^{\alpha} D_{\xi}^{\beta} a(x, \xi)\right|$ is estimated by the right-hand side of $(2.2)$ for all $N \geq N_{n}, \alpha, \beta \in \mathbb{N}_{0}^{d}$, and $\log \left(\frac{\langle(x, \xi)\rangle}{R}\right) \geq \frac{n}{N} \varphi^{*}\left(\frac{N}{n}\right)$.

Now, we investigate the class of pseudodifferential operators for which their symbol is equivalent to zero. In fact, this gives a sufficient condition for a pseudodifferential operator to be $\omega$-regularizing (see Definition 1.20) in terms of formal sums.

Proposition 2.3. If $A$ is a pseudodifferential operator associated to a symbol $a(x, \xi)$ equivalent to zero in $\mathrm{FGS}_{\rho}^{m, \omega}$, then $A$ is $\omega$-regularizing.

Proof. It is enough to show that $a \in \mathcal{S}_{\omega}\left(\mathbb{R}^{2 d}\right)$ since operators with symbols in $\mathcal{S}_{\omega}\left(\mathbb{R}^{2 d}\right.$ ) have kernels in $\mathcal{S}_{\omega}\left(\mathbb{R}^{2 d}\right)$ (Corollary 1.13), and these operators are $\omega$-regularizing by Proposition 1.19. By assumption, there exists $R \geq 1$ such that for all $n \in \mathbb{N}$ there exist $C_{n}>0$ and $N_{n} \in \mathbb{N}$ so that, by $(0.11)$,

$$
\begin{aligned}
\left|D_{x}^{\alpha} D_{\xi}^{\beta} a(x, \xi)\right| & \leq C_{n}\langle(x, \xi)\rangle^{-\rho(|\alpha+\beta|+N)} e^{4 n \rho \varphi^{*}\left(\frac{|\alpha+\beta|+N}{4 n}\right)} e^{m \omega(x, \xi)} \\
& \leq C_{n}\langle(x, \xi)\rangle^{-\rho N} e^{2 n \rho \varphi^{*}\left(\frac{N}{2 n}\right)} e^{2 n \rho \varphi^{*}\left(\frac{|\alpha+\beta|}{2 n}\right)} e^{m \omega(x, \xi)} \\
& \leq C_{n}\left(\frac{\langle(x, \xi)\rangle}{R}\right)^{-\rho N} e^{2 n \rho \varphi^{*}\left(\frac{N}{2 n}\right)} e^{n \varphi^{*}\left(\frac{|\alpha+\beta|}{n}\right)} e^{m \omega(x, \xi)}
\end{aligned}
$$

for all $N \geq N_{n}, \alpha, \beta \in \mathbb{N}_{0}^{d}$, and $\log \left(\frac{\langle(x, \xi)\rangle}{R}\right) \geq \frac{4 n}{N} \varphi^{*}\left(\frac{N}{4 n}\right)$. From Definition $0.3(\alpha)$ there exists $0<\varepsilon<1$ depending on $R$ and on the weight $\omega$ such that

$$
\omega\left(\frac{\langle(x, \xi)\rangle}{R}\right) \geq \varepsilon \omega(\langle(x, \xi)\rangle)-\frac{1}{\varepsilon}, \quad x, \xi \in \mathbb{R}^{d} .
$$

By formulas (0.7) and (0.6), we have

$$
\log \left(\frac{\langle(x, \xi)\rangle}{R}\right) \leq \varphi^{*}(1)+\omega(\langle(x, \xi)\rangle) \leq \varphi^{*}(1)+L+L \omega(x, \xi),
$$

for all $x, \xi \in \mathbb{R}^{d}$. We take $N \geq N_{n}$ depending on $R \geq 1$ and on $x, \xi \in \mathbb{R}^{d}$ such that

$$
\left(\frac{4 n}{N} \varphi^{*}\left(\frac{N}{4 n}\right) \leq\right) \frac{n}{N} \varphi^{*}\left(\frac{N}{n}\right) \leq \log \left(\frac{\langle(x, \xi)\rangle}{R}\right) \leq \frac{n}{N+1} \varphi^{*}\left(\frac{N+1}{n}\right) .
$$

Then, we use Lemma 0.7 to obtain

$$
\begin{aligned}
\left(\left(\frac{\langle(x, \xi)\rangle}{R}\right)^{-N} e^{2 n \varphi^{*}\left(\frac{N}{2 n}\right)}\right)^{\rho} & \leq\left(e^{-n \omega\left(\frac{\langle(x, \xi)\rangle}{R}\right)}\left(\frac{\langle(x, \xi)\rangle}{R}\right)\right)^{\rho} \\
& \leq e^{-n \varepsilon \rho \omega(\langle(x, \xi)\rangle)} e^{\frac{n \rho}{\varepsilon}} e^{\left(\varphi^{*}(1)+L\right) \rho} e^{L \rho \omega(x, \xi)} \\
& \leq e^{\frac{n \rho}{\varepsilon}} e^{\left(\varphi^{*}(1)+L\right) \rho} e^{(-n \varepsilon \rho+L \rho) \omega(x, \xi)}
\end{aligned}
$$


Thus

$$
\left|D_{x}^{\alpha} D_{\xi}^{\beta} a(x, \xi)\right| \leq C_{n} e^{\frac{n \rho}{\varepsilon}} e^{\left(\varphi^{*}(1)+L\right) \rho} e^{n \varphi^{*}\left(\frac{|\alpha+\beta|}{n}\right)} e^{(-n \varepsilon \rho+L \rho+m) \omega(x, \xi)} .
$$

Hence the result follows choosing $n$ large enough.

The reciprocal of Proposition 2.3 is also true for weight functions of the form $\omega(t)=\log ^{s}(1+t)$ for $s \geq 1$. Although we do not consider the case $s=1$ in our setting, the same argument works too. We first need a lemma, which holds for every weight function $\omega$.

Lemma 2.4. If $a \in \bigcap_{m \in \mathbb{R}} \mathrm{GS}_{\rho}^{m, \omega}$, then $a \sim 0$ in $\bigcap_{m \in \mathbb{R}} \mathrm{FGS}_{\rho}^{m, \omega}$.

Proof. Fix $m \in \mathbb{R}$. By assumption, for all $n \in \mathbb{N}$ there exists $C_{n}>0$ (which depends on $m$ ) such that

$$
\left|D_{x}^{\alpha} D_{\xi}^{\beta} a(x, \xi)\right| \leq C_{n}\langle(x, \xi)\rangle^{-\rho|\alpha+\beta|} e^{n \rho \varphi^{*}\left(\frac{|\alpha+\beta|}{n}\right)} e^{(-n L+m) \omega(x, \xi)}
$$

for all $\alpha, \beta \in \mathbb{N}_{0}^{d}$ and $x, \xi \in \mathbb{R}^{d}$. From (0.6) it follows that

$$
-n L \omega(x, \xi) \leq-n \rho \omega(\langle(x, \xi)\rangle)+n L
$$

Moreover, from (0.7) we get

$$
\langle(x, \xi)\rangle^{\rho N} e^{-n \rho \omega(\langle(x, \xi)\rangle)} \leq e^{n \rho \varphi^{*}\left(\frac{N}{n}\right)},
$$

and therefore by $(0.11)$, we have that for all $n \in \mathbb{N}$ there exists $C_{n}^{\prime}>0$ so that

$$
\left|D_{x}^{\alpha} D_{\xi}^{\beta} a(x, \xi)\right| \leq C_{n}^{\prime}\langle(x, \xi)\rangle^{-\rho(|\alpha+\beta|+N)} e^{n \rho \varphi^{*}\left(\frac{|\alpha+\beta|+N}{n}\right)} e^{m \omega(x, \xi)},
$$

for all $N \in \mathbb{N}_{0}, \alpha, \beta \in \mathbb{N}_{0}^{d}$, and $x, \xi \in \mathbb{R}^{d}$. As the argument does not depend on the choice of $m \in \mathbb{R}$, it holds that $a \sim 0$ in $\mathrm{FGS}_{\rho}^{m, \omega}$ for all $m \in \mathbb{R}$.

Proposition 2.5. Let $\omega(t)=\log ^{s}(1+t)$ for $s \geq 1$. If $A$ is an $\omega$-regularizing operator with symbol $a$, then $a \sim 0$ in $\mathrm{FGS}_{\rho}^{m, \omega}$ for all $m \in \mathbb{R}$.

Proof. Since $A$ is $\omega$-regularizing, the symbol $a$ belongs to $\mathcal{S}_{\omega}\left(\mathbb{R}^{2 d}\right)$ by Proposition 1.19 and Corollary 1.13. For $\omega(t)=\log ^{s}(1+t), s \geq 1$, by Example 1.21(b) we have $\mathcal{S}_{\omega}\left(\mathbb{R}^{2 d}\right) \subseteq \bigcap_{m \in \mathbb{R}} \mathrm{GS}_{\rho}^{m, \omega}$. Hence, Lemma 2.4 gives the conclusion. 
Now, we proceed to construct a symbol from an arbitrary formal sum (following the lines of [33, Theorem 3.7]). To do so, we need some kind of partition of unity. Here, we cannot use the estimates as in [33, Lemma 3.6] due to some technical issues. Instead, we consider the usual estimates for ultradifferentiable functions. This is because of the fact that our symbols are defined in the whole $\mathbb{R}^{d}$ for each variable. This is not very restrictive as pointed out in [33, Remark 1.7(1)].

Given a weight function $\omega$, we consider another weight function $\sigma$ such that $\omega\left(t^{1 / \rho}\right)=O(\sigma(t)), t \rightarrow \infty$. Let $\Phi \in \mathcal{D}_{(\sigma)}\left(\mathbb{R}^{2 d}\right)$ be such that

$$
|\Phi| \leq 1, \quad \Phi(t)=1 \text { if }|t| \leq 2, \quad \Phi(t)=0 \text { if }|t| \geq 3 .
$$

Let $\left(j_{n}\right)_{n}$ be an increasing sequence of natural numbers such that $j_{n} / n \rightarrow \infty$ as $n \rightarrow \infty$. For each $j_{n} \leq j<j_{n+1}$, we define

$$
\Psi_{j, n}(x, \xi):=1-\Phi\left(\frac{1}{A_{n, j}}(x, \xi)\right), \quad A_{n, j}=R e^{\frac{n}{j} \varphi_{\omega}^{*}\left(\frac{j}{n}\right)},
$$

for some $R \geq 1$, and all $x, \xi \in \mathbb{R}^{d}$. Notice that $A_{n, j} \rightarrow \infty$ as $j \rightarrow \infty$. If $(x, \xi)$ is in the support of $\Psi_{j, n}$, by $(2.3)$, we have $\left|\frac{1}{A_{n, j}}(x, \xi)\right|>2$. So, if $\Psi_{j, n}(x, \xi) \neq 0$,

$$
\langle(x, \xi)\rangle>2 A_{n, j}
$$

For the estimate of the derivatives of the function $\Psi_{j, n}$, let $C>0$ be as in Lemma 0.10(2). By such lemma, for all $k \in \mathbb{N}$ there exists $C_{k}=C_{k L^{2} C}>0$ such that

$$
\begin{aligned}
\left|D_{x}^{\alpha} D_{\xi}^{\beta} \Psi_{j, n}(x, \xi)\right| & =\left|D_{x}^{\alpha} D_{\xi}^{\beta} \Phi\left(\frac{1}{A_{n, j}}(x, \xi)\right)\right| A_{n, j}^{-|\alpha+\beta|} \\
& \leq C_{k} e^{k L^{2} C \varphi_{\sigma}^{*}\left(\frac{|\alpha+\beta|}{k L^{2} C}\right)} A_{n, j}^{-\rho|\alpha+\beta|} \\
& \leq C_{k} e^{k L^{2} C} e^{k L^{2} \rho \varphi_{\omega}^{*}\left(\frac{|\alpha+\beta|}{k L^{2}}\right)} A_{n, j}^{-\rho|\alpha+\beta|}
\end{aligned}
$$

for all $\alpha, \beta \in \mathbb{N}_{0}^{d}$ and $x, \xi \in \mathbb{R}^{d}$. The points in the support of any derivative of $\Psi_{j, n}$ satisfy $2 \leq\left|\frac{1}{A_{n, j}}(x, \xi)\right| \leq 3$. Thus

$$
2 A_{n, j} \leq\langle(x, \xi)\rangle \leq \sqrt{10} A_{n, j} \leq e^{2} A_{n, j} .
$$

From (2.6) we obtain using (2.7) and (0.10) that for all $k \in \mathbb{N}$ there exists $C_{k}^{\prime}=C_{k} e^{k L^{2} C} e^{\left(k L+k L^{2}\right) \rho}>0$ such that

$$
\left|D_{x}^{\alpha} D_{\xi}^{\beta} \Psi_{j, n}(x, \xi)\right| \leq C_{k}^{\prime}\langle(x, \xi)\rangle^{-\rho|\alpha+\beta|} e^{k \rho \varphi_{\omega}^{*}\left(\frac{|\alpha+\beta|}{k}\right)},
$$


for each $\alpha, \beta \in \mathbb{N}_{0}^{d}$ and $x, \xi \in \mathbb{R}^{d}$. Hence, $\Psi_{j, n} \in \mathrm{GS}_{\rho}^{0, \omega}$.

With the properties of $\Psi_{j, n}$ in (2.4), we are in a position to show the following fundamental result. The proof follows the lines of [33, Theorem 3.7].

Theorem 2.6. Let $\sum a_{j}$ be a formal sum in $\mathrm{FGS}_{\rho}^{m, \omega}$. Then, there exists a global symbol $a \in \mathrm{GS}_{\rho}^{m, \omega}$ such that $a \sim \sum a_{j}$.

Proof. For the functions $\Psi_{j, n}$ defined in (2.4), it follows, by (2.7), that for $(x, \xi)$ in the support of their derivatives we have

$$
\left(e^{2} R\right)^{-\rho j} \leq\langle(x, \xi)\rangle^{-\rho j} e^{n \rho \varphi^{*}\left(\frac{j}{n}\right)} \leq(2 R)^{-\rho j},
$$

for some $R \geq 1$. We check that for all $n \in \mathbb{N}$ there exists $C_{n}>0$ such that

$$
\begin{aligned}
& \left|D_{x}^{\alpha} D_{\xi}^{\beta}\left(\Psi_{j, n}(x, \xi) a_{j}(x, \xi)\right)\right| \\
& \quad \leq C_{n}(2 R)^{-\rho j}\langle(x, \xi)\rangle^{-\rho|\alpha+\beta|} e^{n \rho \varphi^{*}\left(\frac{|\alpha+\beta|}{n}\right)} e^{m \omega(x, \xi)},
\end{aligned}
$$

for all $j \in \mathbb{N}_{0}, \alpha, \beta \in \mathbb{N}_{0}^{d}$, and $\log \left(\frac{\langle(x, \xi)\rangle}{2 R}\right) \geq \frac{n}{j} \varphi^{*}\left(\frac{j}{n}\right)$. This will ensure that $\Psi_{j, n} a_{j} \in \mathrm{GS}_{\rho}^{m, \omega}$ since $\log \left(\frac{\langle(x, \xi)\rangle}{2 R}\right) \leq \frac{n}{j} \varphi^{*}\left(\frac{j}{n}\right)$ implies $\Psi_{j, n}=0$. Choose $\widetilde{p} \in \mathbb{N}_{0}$ so that $2 \leq e^{\rho \widetilde{p}}$. Since $\sum a_{j} \in \mathrm{FGS}_{\rho}^{m, \omega}$, for all $n \in \mathbb{N}$ there exists $C_{n}^{\prime}=C_{2 n L^{\tilde{p}}}^{\prime}>0$ such that

$$
\left|D_{x}^{\alpha} D_{\xi}^{\beta} a_{j}(x, \xi)\right| \leq C_{n}^{\prime}\langle(x, \xi)\rangle^{-\rho(|\alpha+\beta|+j)} e^{2 n L^{\tilde{p}} \rho \varphi^{*}\left(\frac{|\alpha+\beta|+j}{2 n L^{\tilde{p}}}\right)} e^{m \omega(x, \xi)},
$$

for all $j \in \mathbb{N}_{0}, \alpha, \beta \in \mathbb{N}_{0}^{d}$, and $\log \left(\frac{\langle(x, \xi)\rangle}{2 R}\right) \geq \frac{n}{j} \varphi^{*}\left(\frac{j}{n}\right)\left(\geq \frac{2 n L^{\widetilde{p}}}{j} \varphi^{*}\left(\frac{j}{2 n L^{\widetilde{p}}}\right)\right)$. Moreover, since $\Psi_{j, n} \in \mathrm{GS}_{\rho}^{0, \omega}$ there exists $C_{n}^{\prime \prime}=C_{2 n L^{\tilde{p}}}^{\prime \prime}>0$ with

$$
\left|D_{x}^{\alpha} D_{\xi}^{\beta} \Psi_{j, n}(x, \xi)\right| \leq C_{n}^{\prime \prime}\langle(x, \xi)\rangle^{-\rho|\alpha+\beta|} e^{2 n L^{\tilde{p}} \rho \varphi^{*}\left(\frac{|\alpha+\beta|}{2 n L^{\tilde{p}}}\right)},
$$

for all $\alpha, \beta \in \mathbb{N}_{0}^{d}$ and $x, \xi \in \mathbb{R}^{d}$. Then, by Leibniz rule and Lemma 0.8 (with the choice of $\widetilde{p} \in \mathbb{N}_{0}$ ), we have (since $\sum_{\widetilde{\alpha} \leq \alpha ; \widetilde{\beta} \leq \beta}(\underset{\widetilde{\alpha}}{\alpha})\left(\begin{array}{l}\beta \\ \widetilde{\beta}\end{array}\right)=2^{|\alpha+\beta|}$ )

$$
\begin{aligned}
& \left|D_{x}^{\alpha} D_{\xi}^{\beta}\left(\Psi_{j, n}(x, \xi) a_{j}(x, \xi)\right)\right| \\
& \leq \sum_{\widetilde{\alpha} \leq \alpha ; \widetilde{\beta} \leq \beta}\left(\begin{array}{l}
\alpha \\
\widetilde{\alpha}
\end{array}\right)\left(\begin{array}{l}
\beta \\
\widetilde{\beta}
\end{array}\right)\left|D_{x}^{\alpha-\widetilde{\alpha}} D_{\xi}^{\beta-\widetilde{\beta}} \Psi_{j, n}(x, \xi)\right|\left|D_{x}^{\widetilde{\alpha}} D_{\xi}^{\widetilde{\beta}} a_{j}(x, \xi)\right| \\
& \leq C_{n}^{\prime} C_{n}^{\prime \prime}\langle(x, \xi)\rangle^{-\rho(|\alpha+\beta|+j)} e^{2 n L^{\tilde{p}} \rho \varphi^{*}\left(\frac{|\alpha+\beta|+j}{2 n L^{\tilde{p}}}\right)} e^{m \omega(x, \xi)} 2^{|\alpha+\beta|} \\
& \leq C_{n}^{\prime} C_{n}^{\prime \prime} e^{2 n \rho \sum_{s=1}^{\widetilde{p}} L^{s}}\langle(x, \xi)\rangle^{-\rho(|\alpha+\beta|+j)} e^{n \rho \varphi^{*}\left(\frac{|\alpha+\beta|}{n}\right)} e^{n \rho \varphi^{*}\left(\frac{j}{n}\right)} e^{m \omega(x, \xi)},
\end{aligned}
$$


for all $j \in \mathbb{N}_{0}, \alpha, \beta \in \mathbb{N}_{0}^{d}$, and $\log \left(\frac{\langle(x, \xi)\rangle}{2 R}\right) \geq \frac{n}{j} \varphi^{*}\left(\frac{j}{n}\right)\left(\geq \frac{2 n L^{\widetilde{p}}}{j} \varphi^{*}\left(\frac{j}{2 n L^{\widetilde{p}}}\right)\right)$. Then, from formula (2.8) we obtain (2.9).

Let $\left(C_{n}\right)_{n}$ be the sequence of constants appearing in (2.9), and let $\left(j_{n}\right)_{n}$ be the sequence that defines $\Psi_{j, n}$ in (2.4). By induction, we can take such sequence $\left(j_{n}\right)_{n}$ so that $j_{1}:=1, j_{n}<j_{n+1}, j_{n} / n \rightarrow \infty$, and

$$
C_{n+1} \sum_{j=j_{n+1}}^{\infty}(2 R)^{-\rho j} \leq \frac{1}{2} C_{n} \sum_{j=j_{n}}^{j_{n+1}-1}(2 R)^{-\rho j}
$$

We check that

$$
\bar{C}_{n}:=C_{n} \sum_{j=j_{n}}^{j_{n+1}-1}(2 R)^{-\rho j}
$$

satisfies $\bar{C}_{n+1} \leq \frac{1}{2} \bar{C}_{n}$. Indeed,

$$
\bar{C}_{n+1} \leq C_{n+1} \sum_{j=j_{n+1}}^{\infty}(2 R)^{-\rho j} \leq \frac{1}{2} C_{n} \sum_{j=j_{n}}^{j_{n+1}-1}(2 R)^{-\rho j}=\frac{1}{2} \bar{C}_{n}
$$

We prove that

$$
a(x, \xi):=a_{0}(x, \xi)+\sum_{n=1}^{\infty} \sum_{j=j_{n}}^{j_{n+1}-1} \Psi_{j, n}(x, \xi) a_{j}(x, \xi)
$$

is a global symbol in $\mathrm{GS}_{\rho}^{m, \omega}$. First of all, we observe that $a$ defines a locally finite sum: as $j_{n} / n \rightarrow \infty$, for fixed $x, \xi \in \mathbb{R}^{d}$ there exists $n \in \mathbb{N}$ such that $|(x, \xi)| \leq 2 A_{n, j}$ for all $j \geq j_{n}$, thus $\Psi_{j, n}=0$ for all $j \geq j_{n}$. Hence $a$ is well defined, and a $C^{\infty}$ function. By (2.9) we have, for the same sequence of constants $\left(C_{k}\right)_{k}>0$ (according to the definition of $\bar{C}_{k}>0$ in $(2.11)$ ),

$$
\begin{aligned}
& \left|D_{x}^{\alpha} D_{\xi}^{\beta}\left(\sum_{k=n}^{\infty} \sum_{j=j_{k}}^{j_{k+1}-1} \Psi_{j, k}(x, \xi) a_{j}(x, \xi)\right)\right| \\
& \quad \leq \sum_{k=n}^{\infty} \sum_{j=j_{k}}^{j_{k+1}-1}\left|D_{x}^{\alpha} D_{\xi}^{\beta}\left(\Psi_{j, k}(x, \xi) a_{j}(x, \xi)\right)\right| \\
& \quad \leq\langle(x, \xi)\rangle^{-\rho|\alpha+\beta|} e^{m \omega(x, \xi)} \sum_{k=n}^{\infty} C_{k} e^{k \rho \varphi^{*}\left(\frac{|\alpha+\beta|}{k}\right)} \sum_{j=j_{k}}^{j_{k+1}-1}(2 R)^{-\rho j} \\
& \quad \leq\langle(x, \xi)\rangle^{-\rho|\alpha+\beta|} e^{n \rho \varphi^{*}\left(\frac{|\alpha+\beta|}{n}\right)} e^{m \omega(x, \xi)} \sum_{k=n}^{\infty} \bar{C}_{k},
\end{aligned}
$$


for all $\alpha, \beta \in \mathbb{N}_{0}^{d}, \log \left(\frac{\langle(x, \xi)\rangle}{2 R}\right) \geq \frac{n}{j} \varphi^{*}\left(\frac{j}{n}\right)\left(\geq \frac{2 k L^{\widetilde{p}}}{j} \varphi^{*}\left(\frac{j}{2 k L^{\tilde{p}}}\right)\right)$. We recall that if $\log \left(\frac{\langle(x, \xi)\rangle}{2 R}\right) \leq \frac{n}{j} \varphi^{*}\left(\frac{j}{n}\right)$, then $\Psi_{j, n}=0$. As $\sum_{k=n}^{\infty} \bar{C}_{k}$ is a constant depending on $n$ and

$$
a-\sum_{k=n}^{\infty} \sum_{j=j_{k}}^{j_{k+1}-1} \Psi_{j, k} a_{j}=a_{0}+\sum_{k=1}^{n-1} \sum_{j=j_{k}}^{j_{k+1}-1} \Psi_{j, k} a_{j}
$$

is a finite sum of symbols, we obtain $a \in \mathrm{GS}_{\rho}^{m, \omega}$.

We claim that $a \sim \sum a_{j}$. By Definition 2.2, it is enough to show the estimate in (2.2) for $N \geq n j_{n}$. We consider $\log \left(\frac{\langle(x, \xi)\rangle}{\sqrt{10} R}\right) \geq \frac{n}{N} \varphi^{*}\left(\frac{N}{n}\right)$. For arbitrary $j \in \mathbb{N}$ there exists $k \in \mathbb{N}$ such that $j_{k} \leq j<j_{k+1}$. If $k<n$, then $j \leq j_{n}$, and we have

$$
\log \left(\frac{\langle(x, \xi)\rangle}{\sqrt{10} R}\right) \geq \frac{n}{N} \varphi^{*}\left(\frac{N}{n}\right) \geq \frac{1}{j_{n}} \varphi^{*}\left(j_{n}\right) \geq \frac{k}{j} \varphi^{*}\left(\frac{j}{k}\right) .
$$

Therefore, by (2.3), $\Psi_{j, k} \equiv 1$. If $k \geq n$ and $N>j$ we similarly obtain $\Psi_{j, k} \equiv 1$, as

$$
\log \left(\frac{\langle(x, \xi)\rangle}{\sqrt{10} R}\right) \geq \frac{n}{N} \varphi^{*}\left(\frac{N}{n}\right) \geq \frac{k}{j} \varphi^{*}\left(\frac{j}{k}\right) .
$$

Hence, we only deal with the case $k \geq n$ and $j \geq N$. Following the proof of $(2.9)$ (see $(2.10)$ ) we obtain that, for the same sequence $\left(C_{k}\right)_{k}>0$,

$$
\begin{aligned}
& \left|D_{x}^{\alpha} D_{\xi}^{\beta}\left(\Psi_{j, k}(x, \xi) a_{j}(x, \xi)\right)\right| \\
& \quad \leq C_{k}\langle(x, \xi)\rangle^{-\rho(|\alpha+\beta|+N)} e^{k \rho \varphi^{*}\left(\frac{|\alpha+\beta|+N}{k}\right)}\langle(x, \xi)\rangle^{-\rho(j-N)} e^{k \rho \varphi^{*}\left(\frac{j-N}{k}\right)} e^{m \omega(x, \xi)} \\
& \quad \leq C_{k}\langle(x, \xi)\rangle^{-\rho(|\alpha+\beta|+N)} e^{k \rho \varphi^{*}\left(\frac{|\alpha+\beta|+N}{k}\right)}(2 R)^{-\rho(j-N)} e^{m \omega(x, \xi)}
\end{aligned}
$$

for all $N \geq n j_{n}, \alpha, \beta \in \mathbb{N}_{0}^{d}$ and $\log \left(\frac{\langle(x, \xi)\rangle}{\sqrt{10} R}\right) \geq \frac{n}{j} \varphi^{*}\left(\frac{j}{n}\right)$. Since $k \geq n$ and $j \geq N$,

$$
\begin{aligned}
\left|D_{x}^{\alpha} D_{\xi}^{\beta}\left(a-\sum_{j<N} a_{j}\right)\right| & \leq\left|D_{x}^{\alpha} D_{\xi}^{\beta}\left(\sum_{k=1}^{\infty} \sum_{j=j_{k}}^{j_{k+1}-1} \Psi_{j, k} a_{j}-\sum_{j=1}^{N-1} a_{j}\right)\right| \\
& \leq\left|D_{x}^{\alpha} D_{\xi}^{\beta}\left(\sum_{k \geq n} \sum_{j=j_{k}}^{j_{k+1}-1} \Psi_{j, k} a_{j}\right)\right| \\
& \leq \sum_{k \geq n} \sum_{j=j_{k}}^{j_{k+1}-1}\left|D_{x}^{\alpha} D_{\xi}^{\beta}\left(\Psi_{j, k} a_{j}\right)\right|,
\end{aligned}
$$


and, by (2.13), we obtain

$$
\begin{aligned}
& \left|D_{x}^{\alpha} D_{\xi}^{\beta}\left(a-\sum_{j<N} a_{j}\right)\right| \\
& \quad \leq(2 R)^{\rho N}\langle(x, \xi)\rangle^{-\rho(|\alpha+\beta|+N)} e^{m \omega(x, \xi)} \sum_{k \geq n} C_{k} e^{k \rho \varphi^{*}\left(\frac{|\alpha+\beta|+N}{k}\right)} \sum_{j=j_{k} \quad j \geq N}^{j_{k+1}-1}(2 R)^{-\rho j} \\
& \quad \leq(2 R)^{\rho N}\langle(x, \xi)\rangle^{-\rho(|\alpha+\beta|+N)} e^{n \rho \varphi^{*}\left(\frac{|\alpha+\beta|+N}{n}\right)} e^{m \omega(x, \xi)} \sum_{k \geq n} \bar{C}_{k} .
\end{aligned}
$$

This gives the result since $\sum_{k \geq n} \bar{C}_{k}$ is a constant that depends on $n$.

In short, for every $n \in \mathbb{N}$ we write, for $j_{n} \leq j<j_{n+1}$,

$$
\Psi_{j}:=\Psi_{j, n}, \quad \Psi_{0}=1
$$

In what follows, $\tau$ stands for a real number. Let $k \in \mathbb{N}_{0}$ be the smallest natural number satisfying

$$
|\tau|+|1-\tau| \leq 2^{k}
$$

Proceeding as in Lemma 1.4, it is easy to check:

Lemma 2.7. For every $x, y, \xi \in \mathbb{R}^{d}$ and $\tau \in \mathbb{R}$,

$$
\langle(x, y, \xi)\rangle \leq \sqrt{6}\langle\tau\rangle\langle x-y\rangle\langle((1-\tau) x+\tau y, \xi)\rangle .
$$

Proof. We have

$$
\begin{aligned}
& |y|^{2} \leq(|x|+|x-y|)^{2} \leq 2|x|^{2}+2|x-y|^{2} \\
& |x|^{2} \leq(|x-\tau(x-y)|+|\tau(x-y)|)^{2} \leq 2|x-\tau(x-y)|^{2}+2|\tau(x-y)|^{2} .
\end{aligned}
$$

Then,

$$
\begin{aligned}
|x|^{2}+|y|^{2} & \leq 3|x|^{2}+2|x-y|^{2} \\
& \leq 6|x-\tau(x-y)|^{2}+6|\tau|^{2}|x-y|^{2}+2|x-y|^{2} \\
& \leq 6|(1-\tau) x+\tau y|^{2}+6\left(1+|\tau|^{2}\right)|x-y|^{2} .
\end{aligned}
$$

Therefore, it is easy to see that

$$
\frac{1+|x|^{2}+|y|^{2}+|\xi|^{2}}{\left(1+|x-y|^{2}\right)\left(1+|(1-\tau) x+\tau y|^{2}+|\xi|^{2}\right)} \leq 6\left(1+|\tau|^{2}\right) .
$$


Given $m \in \mathbb{R}$ we denote for $k \in \mathbb{N}_{0}$ as in (2.16),

$$
m^{\prime}=m L^{k} \text {. }
$$

We observe that $m^{\prime}=m$ if and only if $0 \leq \tau \leq 1$.

The next result generalizes Example 1.5.

Lemma 2.8. If $b(x, \xi) \in \mathrm{GS}_{\rho}^{m, \omega}$ and $\tau \in \mathbb{R}$, then

$$
a(x, y, \xi):=b((1-\tau) x+\tau y, \xi)
$$

is a global amplitude in $\mathrm{GA}_{\rho}^{\max \left\{0, m^{\prime}\right\}, \omega}$.

Proof. We take $\widetilde{p} \in \mathbb{N}$ such that $\max \left\{|1-\tau|,|\tau|,(\sqrt{6}\langle\tau\rangle)^{\rho}\right\} \leq e^{\rho \widetilde{p}}$. By assumption, for all $\lambda>0$ there exists $C_{\lambda}>0$ such that

$$
\begin{aligned}
\left|D_{x}^{\alpha} D_{y}^{\gamma} D_{\xi}^{\beta} a(x, y, \xi)\right| \leq & |1-\tau|^{|\alpha|}|\tau|^{|\gamma|} C_{\lambda}\langle((1-\tau) x+\tau y, \xi)\rangle^{-\rho|\alpha+\gamma+\beta|} \times \\
& \times e^{\lambda L^{2 \widetilde{p}} \rho \varphi^{*}\left(\frac{|\alpha+\gamma+\beta|}{\lambda L^{2 \widetilde{p}}}\right)} e^{m \omega((1-\tau) x+\tau y, \xi)}
\end{aligned}
$$

for all $\alpha, \gamma, \beta \in \mathbb{N}_{0}^{d}, x, y, \xi \in \mathbb{R}^{d}$. We use Lemma 2.7 to get

$$
\langle((1-\tau) x+\tau y, \xi)\rangle^{-\rho|\alpha+\gamma+\beta|} \leq(\sqrt{6}\langle\tau\rangle)^{\rho|\alpha+\gamma+\beta|}\left(\frac{\langle x-y\rangle}{\langle(x, y, \xi)\rangle}\right)^{\rho|\alpha+\gamma+\beta|} .
$$

The choice of $\widetilde{p} \in \mathbb{N}$ gives $|1-\tau|^{|\alpha|}|\tau|^{|\gamma|}(\sqrt{6}\langle\tau\rangle)^{\rho|\alpha+\gamma+\beta|} \leq e^{2 \rho \widetilde{p}|\alpha+\gamma+\beta|}$. Then, by $(0.10)$, we get

$$
\left[e^{2 \widetilde{p}|\alpha+\gamma+\beta|} e^{\lambda L^{2 \widetilde{p}} \varphi^{*}\left(\frac{|\alpha+\gamma+\beta|}{\lambda L^{2 \widetilde{p}}}\right)}\right]^{\rho} \leq e^{\lambda \rho \varphi^{*}\left(\frac{|\alpha+\gamma+\beta|}{\lambda}\right)} e^{\lambda \rho \sum_{j=1}^{2 \widetilde{p}} L^{j}} .
$$

Finally, since $\omega$ is radial and increasing, using $k$ times condition $(\alpha)$ of Definition 0.3 , we conclude for $m \geq 0$

$$
e^{m \omega((1-\tau) x+\tau y, \xi)} \leq e^{m \omega\left(2^{k}(x, y, \xi)\right)} \leq e^{m^{\prime} \omega(x, y, \xi)} e^{m L^{k}+m L^{k-1}+\cdots+m L} .
$$

Since $\Psi_{j}$ as in (2.15) is a global symbol in $\mathrm{GS}_{\rho}^{0, \omega}$, it follows that

Corollary 2.9. Let $\Psi_{j}(x, \xi)$ be as in $(2.15)$. Then $\Psi_{j}((1-\tau) x+\tau y, \xi) \in \mathrm{GA}_{\rho}^{0, \omega}$ for all $\tau \in \mathbb{R}$. Moreover, for all $\lambda>0$ there exists $C_{\lambda}>0$ such that

$\left|D_{x}^{\alpha} D_{y}^{\gamma} D_{\xi}^{\beta} \Psi_{j}((1-\tau) x+\tau y, \xi)\right| \leq C_{\lambda}\langle((1-\tau) x+\tau y, \xi)\rangle^{-\rho|\alpha+\gamma+\beta|} e^{\lambda \rho \varphi^{*}\left(\frac{|\alpha+\gamma+\beta|}{\lambda}\right)}$ for all $\alpha, \gamma, \beta \in \mathbb{N}_{0}^{d}$ and $x, y, \xi \in \mathbb{R}^{d}$. 
Lemma 2.10. Let $a(x, y, \xi)$ be an amplitude in $\mathrm{GA}_{\rho}^{m, \omega}$ and let $A$ be the associated pseudodifferential operator. For each $f \in \mathcal{S}_{\omega}\left(\mathbb{R}^{d}\right)$, we have

$$
A(f)=\sum_{j=0}^{\infty} A_{j}(f) \text { in } \mathcal{S}_{\omega}\left(\mathbb{R}^{d}\right)
$$

where $A_{j}$ is the pseudodifferential operator defined by the amplitude

$$
\left(\Psi_{j}-\Psi_{j+1}\right)((1-\tau) x+\tau y, \xi) a(x, y, \xi), \quad j \in \mathbb{N}_{0} .
$$

Proof. By Corollary 2.9, $\left(\Psi_{j}-\Psi_{j+1}\right)((1-\tau) x+\tau y, \xi) a(x, y, \xi) \in \mathrm{GA}_{\rho}^{m, \omega}$ for $j_{n} \leq j<j_{n+1}$. Since $A_{n, N+1} \rightarrow \infty$ as $N \rightarrow \infty$, for each $f \in \mathcal{S}_{\omega}\left(\mathbb{R}^{d}\right)$ we have

$$
\begin{aligned}
& \sum_{j=0}^{\infty} A_{j}(f)(x) \\
& \quad=\sum_{j=0}^{\infty} \iint e^{i(x-y) \cdot \xi}\left(\Psi_{j}-\Psi_{j+1}\right)((1-\tau) x+\tau y, \xi) a(x, y, \xi) f(y) d y d \xi \\
& \quad=\lim _{N \rightarrow \infty} \iint e^{i(x-y) \cdot \xi}\left(1-\Psi_{N+1}((1-\tau) x+\tau y, \xi)\right) a(x, y, \xi) f(y) d y d \xi .
\end{aligned}
$$

We show that this limit is, for all $\tau \in \mathbb{R}$, equal to $A$ in $L\left(\mathcal{S}_{\omega}\left(\mathbb{R}^{d}\right), \mathcal{S}_{\omega}^{\prime}\left(\mathbb{R}^{d}\right)\right)$. We recall that

$$
\left(1-\Psi_{N+1}\right)((1-\tau) x+\tau y, \xi)=\Phi\left(\frac{((1-\tau) x+\tau y, \xi)}{A_{n, N+1}}\right)
$$

and $\Phi(0)=1$, being $\Phi \in \mathcal{D}_{(\sigma)}\left(\mathbb{R}^{2 d}\right)$ the function in (2.3) with $\omega\left(t^{1 / \rho}\right)=$ $O(\sigma(t)), t \rightarrow \infty$. Since $\mathcal{S}_{\omega}\left(\mathbb{R}^{d}\right)$ is Fréchet-Montel, it is enough that for each $f, g \in \mathcal{S}_{\omega}\left(\mathbb{R}^{d}\right)$,

$$
\iiint e^{i(x-y) \cdot \xi}\left(\Phi\left(\frac{(1-\tau) x+\tau y, \xi}{k}\right)-1\right) a(x, y, \xi) f(y) g(x) d y d \xi d x \rightarrow 0
$$

as $k \rightarrow \infty$. We integrate by parts with formula (1.6) for some power $s \in \mathbb{N}$ determined later of the ultradifferential operator $G(D)$. Then, the integrand in the left-hand side of (2.19) equals

$$
\begin{aligned}
e^{i(x-y) \cdot \xi} & \frac{1}{G^{s}(\xi)} G^{s}\left(D_{y}\right)\left\{\left(\Phi\left(\frac{(1-\tau) x+\tau y, \xi}{k}\right)-1\right) a(x, y, \xi) f(y) g(x)\right\} \\
= & e^{i(x-y) \cdot \xi} \frac{1}{G^{s}(\xi)} \sum_{\eta \in \mathbb{N}_{0}^{d}} b_{\eta} \sum_{\eta_{1}+\eta_{2}+\eta_{3}=\eta} \frac{\eta !}{\eta_{1} ! \eta_{2} ! \eta_{3} !} \times \\
& \times\left(\frac{\tau}{k}\right)^{\left|\eta_{1}\right|} D_{y}^{\eta_{1}}\left(\Phi\left(\frac{(1-\tau) x+\tau y, \xi}{k}\right)-1\right) D_{y}^{\eta_{2}} a(x, y, \xi) D_{y}^{\eta_{3}} f(y) g(x) .
\end{aligned}
$$


Therefore, the integral in (2.19) is equal to

$$
\begin{aligned}
& \sum_{\eta \in \mathbb{N}_{0}^{d}} b_{\eta} \sum_{\eta_{1}+\eta_{2}+\eta_{3}=\eta} \frac{\eta !}{\eta_{1} ! \eta_{2} ! \eta_{3} !}\left(\frac{\tau}{k}\right)^{\left|\eta_{1}\right|} \iiint e^{i(x-y) \cdot \xi} \frac{1}{G^{s}(\xi)} \times \\
& \quad \times D_{y}^{\eta_{1}}\left(\Phi\left(\frac{(1-\tau) x+\tau y, \xi}{k}\right)-1\right) D_{y}^{\eta_{2}} a(x, y, \xi) D_{y}^{\eta_{3}} f(y) g(x) d y d \xi d x .
\end{aligned}
$$

From Corollaries 0.23 and 0.20 there are $C_{1}, C_{2}, C_{3}>0$ (depending only on $G$ ) such that for all $\eta \in \mathbb{N}_{0}^{d}$ and $\xi \in \mathbb{R}^{d}$

$$
\left|b_{\eta}\right| \leq e^{s C_{1}} e^{-s C_{1} \varphi^{*}\left(\frac{|\eta|}{s C_{1}}\right)}, \quad\left|\frac{1}{G^{s}(\xi)}\right| \leq C_{3}^{s} e^{-s C_{2} \omega(\xi)} .
$$

It follows from Lemma 1.4 (and (0.10)) that for all $\lambda>0$ there exists $C_{\lambda}>0$ such that

$$
\left|D_{y}^{\eta_{2}} a(x, y, \xi)\right| \leq C_{\lambda} e^{\lambda L^{3} \varphi^{*}\left(\frac{\left|\eta_{2}\right|}{\lambda L^{3}}\right)} e^{m \omega(x, y, \xi)} .
$$

Since $f, g \in \mathcal{S}_{\omega}\left(\mathbb{R}^{d}\right)$, there exist $C_{\lambda, m}^{\prime}, C_{m}>0$ such that

$$
\begin{aligned}
\left|D_{y}^{\eta_{3}} f(y)\right| & \leq C_{\lambda, m}^{\prime} e^{\lambda L^{3} \varphi^{*}\left(\frac{\left|\eta_{3}\right|}{\lambda L^{3}}\right)} e^{-(m L+1) \omega(y)} ; \\
|g(x)| & \leq C_{m} e^{-(m L+1) \omega(x)} .
\end{aligned}
$$

Now, for $\eta_{1}=0$ we have that $\Phi \equiv 1$ if $|((1-\tau) x+\tau y, \xi)| \leq 2 k$, and for $\left|\eta_{1}\right|>0$ it follows that $D_{y}^{\eta_{1}}\left(\Phi\left(\frac{(1-\tau) x+\tau y, \xi}{k}\right)-1\right)=D_{y}^{\eta_{1}} \Phi\left(\frac{(1-\tau) x+\tau y, \xi}{k}\right)$ is zero for $|((1-\tau) x+\tau y, \xi)| \leq 2 k$; therefore we assume that $|((1-\tau) x+\tau y, \xi)|>2 k$. We then have

$$
1 \leq \frac{1}{2 k}|((1-\tau) x+\tau y, \xi)| \leq \frac{1}{k}(|1-\tau|+|\tau|)(|x|+1)(|y|+1)(|\xi|+1) .
$$

As $\Phi \in \mathcal{D}_{(\sigma)}\left(\mathbb{R}^{2 d}\right) \subseteq \mathcal{D}_{(\omega)}\left(\mathbb{R}^{2 d}\right)$, by $(0.10)$, there exists $C_{\lambda}^{\prime \prime}>0$ such that

$$
|\tau|^{\left|\eta_{1}\right|}\left|D_{y}^{\eta_{1}}\left(\Phi\left(\frac{(1-\tau) x+\tau y, \xi}{k}\right)-1\right)\right| \leq C_{\lambda}^{\prime \prime} e^{\lambda L^{3} \varphi^{*}\left(\frac{\left|\eta_{1}\right|}{\lambda L^{3}}\right)}, \quad \eta_{1} \in \mathbb{N}_{0}^{d} .
$$

For $m \geq 0$ (if $m<0$, then $m \omega(x, y, \xi)<0$ ), formula (0.4) gives

$$
m \omega(x, y, \xi) \leq m L \omega(x)+m L \omega(y)+m L \omega(\xi)+m L .
$$

If $s \in \mathbb{N}$ satisfies $s C_{2} \geq m L+1$, we get $e^{\left(-s C_{2}+m L\right) \omega(\xi)} \leq e^{-\omega(\xi)}$ and therefore the integrals in (2.19) are convergent by condition $(\gamma)$ of Definition 0.3. On the other hand, from Lemma 0.8 , since $\sum \frac{\eta !}{\eta_{1} ! \eta_{2} ! \eta_{3} !}=3^{|\eta|} \leq e^{2|\eta|}$, we have

$$
\sum_{\eta_{1}+\eta_{2}+\eta_{3}=\eta} \frac{\eta !}{\eta_{1} ! \eta_{2} ! \eta_{3} !} e^{\lambda L^{3} \varphi^{*}\left(\frac{\left|\eta_{1}\right|}{\lambda L^{3}}\right)} e^{\lambda L^{3} \varphi^{*}\left(\frac{\left|\eta_{2}\right|}{\lambda L^{3}}\right)} e^{\lambda L^{3} \varphi^{*}\left(\frac{\left|\eta_{3}\right|}{\lambda L^{3}}\right)} \leq e^{\lambda L \varphi^{*}\left(\frac{|\eta|}{\lambda L}\right)} e^{\lambda L^{2}+\lambda L^{3}} .
$$


Now, the series

$$
\sum_{\eta \in \mathbb{N}_{0}^{d}} e^{-s C_{1} \varphi^{*}\left(\frac{|\eta|}{s C_{1}}\right)} e^{\lambda L \varphi^{*}\left(\frac{|\eta|}{\lambda L}\right)}
$$

converges provided $\lambda>s C_{1}$ (see (1.14)). Thus, there exists $C>0$ such that $\left|\iiint e^{i(x-y) \cdot \xi}\left(\Phi\left(\frac{(1-\tau) x+\tau y, \xi}{k}\right)-1\right) a(x, y, \xi) f(y) g(x) d y d \xi d x\right| \leq C \frac{1}{k} \rightarrow 0$ and hence (2.19) is satisfied, which proves the result.

Lemma 2.11. Let $\sum p_{j}$ be a formal sum in $\mathrm{FGS}_{\rho}^{m, \omega}$. Let $\left(C_{n}\right)_{n}$ and $\left(C_{n}^{\prime}\right)_{n}$ be the sequences of constants that appear in Definition 2.1 and in Corollary 2.9. Assume that the sequence $\left(j_{n}\right)_{n}$ in the proof of Theorem 2.6 satisfies in addition

$$
\frac{n}{j} \varphi^{*}\left(\frac{j}{n}\right) \geq \max \left\{n, \log \left(C_{2 n L^{\widetilde{p}+1}}\right), \log \left(C_{n L^{\tilde{p}+1}}^{\prime}\right)\right\} \quad \text { for } j_{n} \leq j<j_{n+1},
$$

where $\widetilde{p} \in \mathbb{N}$ is so that $3\langle\tau\rangle \leq e^{\widetilde{p}}$. For

$$
p(x, \xi):=\sum_{j=0}^{\infty} \Psi_{j}(x, \xi) p_{j}(x, \xi)
$$

its corresponding pseudodifferential operator $P$ is, in $L\left(\mathcal{S}_{\omega}\left(\mathbb{R}^{d}\right), \mathcal{S}_{\omega}^{\prime}\left(\mathbb{R}^{d}\right)\right)$, the limit of the sequence of operators

$$
S_{N, \tau}: \mathcal{S}_{\omega}\left(\mathbb{R}^{d}\right) \rightarrow \mathcal{S}_{\omega}\left(\mathbb{R}^{d}\right), \quad N \in \mathbb{N},
$$

where each $S_{N, \tau}, N \in \mathbb{N}$, denotes a pseudodifferential operator with amplitude

$$
\sum_{j=0}^{N}\left(\Psi_{j}-\Psi_{j+1}\right)((1-\tau) x+\tau y, \xi) \sum_{l=0}^{j} p_{l}((1-\tau) x+\tau y, \xi)
$$

in $\mathrm{GA}_{\rho}^{\max \left\{0, m^{\prime}\right\}, \omega}$, where $m^{\prime}$ is as in $(2.17)$.

Proof. For each $j \in \mathbb{N}_{0}$, we check that

$$
\left(\left(\Psi_{j}-\Psi_{j+1}\right) \sum_{l=0}^{j} p_{l}\right)((1-\tau) x+\tau y, \xi)=\sum_{l=0}^{j}\left(\left(\Psi_{j}-\Psi_{j+1}\right) p_{l}\right)((1-\tau) x+\tau y, \xi)
$$

is a global amplitude in $\mathrm{GA}_{\rho}^{\max \left\{0, m^{\prime}\right\}, \omega}$. We choose $\widetilde{p}_{1} \in \mathbb{N}$ so that

$$
\max \left\{2,2|1-\tau|, 2|\tau|,(\sqrt{6}\langle\tau\rangle)^{\rho}\right\} \leq e^{\rho \widetilde{p}_{1}}
$$


From (2.7) we assume (since $0 \leq l \leq j$ )

$$
\left(A_{2 n L^{2 \widetilde{p}_{1}}, l} \leq\right) 2 A_{n, j} \leq\langle((1-\tau) x+\tau y, \xi)\rangle \leq \sqrt{10} A_{n, j} .
$$

By Definition 2.1 and the first part of Corollary 2.9, for all $n \in \mathbb{N}$ we have for the sequences as in the statement of the lemma,

$$
\begin{aligned}
& \left|D_{x}^{\alpha} D_{y}^{\gamma} D_{\xi}^{\beta}\left(\left(\Psi_{j}-\Psi_{j+1}\right) p_{l}\right)((1-\tau) x+\tau y, \xi)\right| \\
& \leq \sum_{\widetilde{\alpha} \leq \alpha ; \widetilde{\gamma} \leq \gamma ; \widetilde{\beta} \leq \beta}\left(\begin{array}{c}
\alpha \\
\widetilde{\alpha}
\end{array}\right)\left(\begin{array}{l}
\gamma \\
\widetilde{\gamma}
\end{array}\right)\left(\begin{array}{c}
\beta \\
\widetilde{\beta}
\end{array}\right)\left|D_{x}^{\widetilde{\alpha}} D_{y}^{\widetilde{\gamma}} D_{\xi}^{\widetilde{\beta}}\left(\Psi_{j}-\Psi_{j+1}\right)((1-\tau) x+\tau y, \xi)\right| \times \\
& \times\left|D_{x}^{\alpha-\widetilde{\alpha}} D_{y}^{\gamma-\widetilde{\gamma}} D_{\xi}^{\beta-\widetilde{\beta}} p_{l}((1-\tau) x+\tau y, \xi)\right| \\
& \leq \sum_{\widetilde{\alpha} \leq \alpha ; \widetilde{\gamma} \leq \gamma ; \widetilde{\beta} \leq \beta}\left(\begin{array}{c}
\alpha \\
\widetilde{\alpha}
\end{array}\right)\left(\begin{array}{l}
\gamma \\
\widetilde{\gamma}
\end{array}\right)\left(\begin{array}{c}
\beta \\
\widetilde{\beta}
\end{array}\right)|1-\tau|^{|\widetilde{\alpha}|}|\tau|^{|\widetilde{\gamma}|} C_{n L^{2 \widetilde{p}_{1}}}^{\prime} \times \\
& \times\left(\frac{\langle x-y\rangle}{\langle(x, y, \xi)\rangle}\right)^{\rho|\widetilde{\alpha}+\widetilde{\gamma}+\widetilde{\beta}|} e^{n L^{2 \tilde{p}_{1}} \rho \varphi^{*}\left(\frac{|\widetilde{\alpha}+\tilde{\gamma}+\widetilde{\beta}|}{n L^{2 \tilde{p}} 1}\right)}|1-\tau|^{|\alpha-\widetilde{\alpha}|}|\tau|^{|\gamma-\widetilde{\gamma}|} C_{2 n L^{2 \widetilde{p}_{1}} \times} \\
& \times\langle((1-\tau) x+\tau y, \xi)\rangle^{-\rho(|\alpha-\widetilde{\alpha}+\gamma-\widetilde{\gamma}+\beta-\widetilde{\beta}|+l)} \times \\
& \times e^{2 n L^{2 \widetilde{p}_{1}} \rho \varphi^{*}\left(\frac{|\alpha-\tilde{\alpha}+\gamma-\tilde{\gamma}+\beta-\widetilde{\beta}|+l}{2 n L^{2 \tilde{p}_{1}}}\right)} e^{m \omega((1-\tau) x+\tau y, \xi)} .
\end{aligned}
$$

We first notice that, since $(2 R)^{-\rho l} \leq 1$, by $(0.11)$,

$$
\langle((1-\tau) x+\tau y, \xi)\rangle^{-\rho l} e^{2 n L^{2 \widetilde{p}_{1}} \rho \varphi^{*}\left(\frac{|\alpha-\tilde{\alpha}+\gamma-\tilde{\gamma}+\beta-\widetilde{\beta}|+l}{2 n L^{2 \tilde{p}_{1}}}\right)} \leq e^{n L^{2 \widetilde{p}_{1}} \rho \varphi^{*}\left(\frac{|\alpha-\tilde{\alpha}+\gamma-\tilde{\gamma}+\beta-\widetilde{\beta}|}{n L^{2 \tilde{p}_{1}}}\right) .}
$$

By Lemma 2.7, we deduce

$$
\begin{aligned}
& \langle((1-\tau) x+\tau y, \xi)\rangle^{-\rho|\alpha-\widetilde{\alpha}+\gamma-\widetilde{\gamma}+\beta-\widetilde{\beta}|} \\
& \quad \leq(\sqrt{6}\langle\tau\rangle)^{\rho|\alpha+\gamma+\beta|}\left(\frac{\langle x-y\rangle}{\langle(x, y, \xi)\rangle}\right)^{\rho|\alpha-\widetilde{\alpha}+\gamma-\widetilde{\gamma}+\beta-\widetilde{\beta}|} .
\end{aligned}
$$

Then, from Lemma 0.8 , since $\sum(\underset{\widetilde{\alpha}}{\alpha})(\underset{\widetilde{\gamma}}{\gamma})\left(\begin{array}{c}\beta \\ \widetilde{\beta}\end{array}\right)=2^{|\alpha+\gamma+\beta|}$, we have by the choice of $\widetilde{p}_{1} \in \mathbb{N}$,

$$
\begin{aligned}
& (\sqrt{6}\langle\tau\rangle)^{\rho|\alpha+\gamma+\beta|}|1-\tau|^{|\alpha|}|\tau|^{|\gamma|} \sum_{\widetilde{\alpha} \leq \alpha ; \widetilde{\gamma} \leq \gamma ; \widetilde{\beta} \leq \beta}\left(\begin{array}{c}
\alpha \\
\widetilde{\alpha}
\end{array}\right)\left(\begin{array}{c}
\gamma \\
\widetilde{\gamma}
\end{array}\right)\left(\begin{array}{c}
\beta \\
\widetilde{\beta}
\end{array}\right) \times \\
& \times e^{n L^{2 \widetilde{p}_{1}} \rho \varphi^{*}\left(\frac{|\tilde{\alpha}+\tilde{\gamma}+\widetilde{\beta}|}{n L^{2} \tilde{p}_{1}}\right)} e^{n L^{2 \widetilde{p}_{1}} \rho \varphi^{*}\left(\frac{|\alpha-\tilde{\alpha}+\gamma-\tilde{\gamma}+\beta-\widetilde{\beta}|}{n L^{2} \tilde{p}_{1}}\right)} \\
& \leq(\sqrt{6}\langle\tau\rangle)^{\rho|\alpha+\gamma+\beta|}(2|1-\tau|)^{|\alpha|}(2|\tau|)^{|\gamma|} 2^{|\beta|} e^{n L^{2 \tilde{p}_{1}} \rho \varphi^{*}\left(\frac{|\alpha+\gamma+\beta|}{n L^{2 \tilde{p}} 1}\right)} \\
& \leq e^{n \rho \varphi^{*}\left(\frac{|\alpha+\gamma+\beta|}{n}\right)} e^{n \rho \sum_{t=1}^{2 \widetilde{p}_{1}} L^{t}} .
\end{aligned}
$$


By (2.18), we obtain that $\sum_{l=0}^{j}\left(\Psi_{j}-\Psi_{j+1}\right) p_{l} \in \mathrm{GA}_{\rho}^{\max \left\{0, m^{\prime}\right\}, \omega}$. Hence, the function

$$
\sum_{j=0}^{N}\left(\Psi_{j}-\Psi_{j+1}\right)\left(\sum_{l=0}^{j} p_{l}\right)=\sum_{j=0}^{N} \Psi_{j} p_{j}-\Psi_{N+1} \sum_{l=0}^{N} p_{l}
$$

is a global amplitude in $\mathrm{GA}_{\rho}^{\max \left\{0, m^{\prime}\right\}, \omega}$.

Now, we prove that $S_{N, \tau}$ converges to the operator $P$ in $L\left(\mathcal{S}_{\omega}\left(\mathbb{R}^{d}\right), \mathcal{S}_{\omega}^{\prime}\left(\mathbb{R}^{d}\right)\right)$ when $N \rightarrow \infty$. Since $\mathcal{S}_{\omega}\left(\mathbb{R}^{d}\right)$ is a Fréchet-Montel space, it is enough to show that, for all $f, g \in \mathcal{S}_{\omega}\left(\mathbb{R}^{d}\right)$,

$$
\left\langle\left(S_{N, \tau}-P\right) f, g\right\rangle \rightarrow 0, \quad \text { as } N \rightarrow \infty .
$$

Since $P$ and $S_{N, \tau}, N \in \mathbb{N}$, act continuously from $\mathcal{S}_{\omega}\left(\mathbb{R}^{d}\right)$ into $\mathcal{S}_{\omega}\left(\mathbb{R}^{d}\right)$, we have

$$
\begin{aligned}
& \left\langle\left(S_{N, \tau}-P\right) f, g\right\rangle=\int\left(S_{N, \tau}-P\right) f(x) g(x) d x \\
& \quad=\int\left(\iint e^{i(x-y) \cdot \xi}\left(\left\{\sum_{j=0}^{N} \Psi_{j} p_{j}-\Psi_{N+1} \sum_{l=0}^{N} p_{l}\right\}-p\right) f(y) d y d \xi\right) g(x) d x
\end{aligned}
$$

for every $f, g \in \mathcal{S}_{\omega}\left(\mathbb{R}^{d}\right)$, where $\Psi_{j}, \Psi_{N+1}, p_{j}, p_{l}$, and $p$ are evaluated at $((1-$ $\tau) x+\tau y, \xi)$. We prove that, for each $f, g \in \mathcal{S}_{\omega}\left(\mathbb{R}^{d}\right)$,

$$
\begin{aligned}
& \text { a) } \int\left(\iint e^{i(x-y) \cdot \xi}\left(\sum_{j=N+1}^{\infty} \Psi_{j} p_{j}\right) f(y) d y d \xi\right) g(x) d x \text {, and } \\
& \text { b) } \int\left(\iint e^{i(x-y) \cdot \xi}\left(\Psi_{N+1} \sum_{l=0}^{N} p_{l}\right) f(y) d y d \xi\right) g(x) d x
\end{aligned}
$$

tend to zero when $N \rightarrow \infty$.

Let us show that $a$ ) goes to zero. We integrate by parts with formula (1.6) for some $s \in \mathbb{N}$ determined later. The integrand in $a$ ) equals

$$
\begin{aligned}
& e^{i(x-y) \cdot \xi} \frac{1}{G^{s}(\xi)} G^{s}\left(D_{y}\right)\left(\sum_{j=N+1}^{\infty} \Psi_{j} \cdot p_{j} \cdot f(y)\right) \\
& =e^{i(x-y) \cdot \xi} \frac{1}{G^{s}(\xi)} \sum_{\eta \in \mathbb{N}_{0}^{d}} b_{\eta} \sum_{\eta_{1}+\eta_{2}+\eta_{3}=\eta} \frac{\eta !}{\eta_{1} ! \eta_{2} ! \eta_{3} !} \sum_{j=N+1}^{\infty} \tau^{\left|\eta_{1}+\eta_{2}\right|} D_{y}^{\eta_{1}} \Psi_{j} D_{y}^{\eta_{2}} p_{j} D_{y}^{\eta_{3}} f(y) .
\end{aligned}
$$


Hence, we reformulate $a$ ) by

$$
\begin{aligned}
& \int\left(\int \frac{1}{G^{s}(\xi)} \sum_{\eta \in \mathbb{N}_{0}^{d}} b_{\eta} \sum_{\eta_{1}+\eta_{2}+\eta_{3}=\eta} \frac{\eta !}{\eta_{1} ! \eta_{2} ! \eta_{3} !} \tau^{\left|\eta_{1}+\eta_{2}\right|} \times\right. \\
& \left.\times\left(\int e^{i(x-y) \cdot \xi} \sum_{j=N+1}^{\infty} D_{y}^{\eta_{1}} \Psi_{j} D_{y}^{\eta_{2}} p_{j} D_{y}^{\eta_{3}} f(y) d y\right) d \xi\right) g(x) d x .
\end{aligned}
$$

When $\Psi_{j} \neq 0$, and $j_{n} \leq j<j_{n+1}$, we have $\log \left(\frac{\langle((1-\tau) x+\tau y, \xi)\rangle}{2 R}\right) \geq \frac{n}{j} \varphi^{*}\left(\frac{j}{n}\right)$ by (2.5). By Corollary 2.9, for each $n \in \mathbb{N}$ we have (for $C_{n}^{\prime}=C_{n L^{\tilde{p}+1}}^{\prime}>0$ as in the statement of the lemma),

$$
\left|D_{y}^{\eta_{1}} \Psi_{j}((1-\tau) x+\tau y, \xi)\right| \leq C_{n}^{\prime} e^{n L^{\widetilde{p}+1} \varphi^{*}\left(\frac{\left|\eta_{1}\right|}{n L^{\tilde{p}+1}}\right)} .
$$

Moreover, for that $n \in \mathbb{N}$, we have from Definition 2.1, by $(0.11)$, (we denote $\left.C_{n}=C_{2 n L^{\widetilde{p}+1}}>0\right)$,

$$
\begin{aligned}
& \left|D_{y}^{\eta_{2}} p_{j}((1-\tau) x+\tau y, \xi)\right| \\
& \quad \leq C_{n} e^{2 n L^{\widetilde{p}+1} \rho \varphi^{*}\left(\frac{\left|\eta_{2}\right|+j}{2 n L^{\tilde{p}}+1}\right)}\langle((1-\tau) x+\tau y, \xi)\rangle^{-\rho\left(\left|\eta_{2}\right|+j\right)} e^{m \omega((1-\tau) x+\tau y, \xi)} \\
& \quad \leq C_{n} e^{n L^{\widetilde{p}+1} \varphi^{*}\left(\frac{\left|\eta_{2}\right|}{n L^{\tilde{p}+1}}\right)} e^{n L^{\widetilde{p}+1} \rho \varphi^{*}\left(\frac{j}{n L^{\tilde{p}+1}}\right)}\langle((1-\tau) x+\tau y, \xi)\rangle^{-\rho j} e^{m \omega((1-\tau) x+\tau y, \xi)} \\
& \quad \leq C_{n} e^{n L^{\widetilde{p}+1} \varphi^{*}\left(\frac{\left|\eta_{2}\right|}{n L^{\tilde{p}+1}}\right)}(2 R)^{-\rho j} e^{m \omega((1-\tau) x+\tau y, \xi)} .
\end{aligned}
$$

We may assume that $m \geq 0$ (otherwise, the proof is simpler). Property $(\gamma)$ of $\omega$ yields that there exists $C>0$ such that $\langle t\rangle \leq C e^{\omega(\langle t\rangle)}, t \in \mathbb{R}^{d}$. By $(0.6)$, we obtain, according to the support of $\Psi_{j}$ (see (2.5)),

$$
\begin{aligned}
e^{m \omega((1-\tau) x+\tau y, \xi)} & \leq e^{(m+3) \omega(\langle((1-\tau) x+\tau y, \xi)\rangle)} e^{-3 \omega(\langle((1-\tau) x+\tau y, \xi)\rangle)} \\
& \leq e^{(m+3) L \omega((1-\tau) x+\tau y, \xi)} e^{(m+3) L} C^{3}\langle((1-\tau) x+\tau y, \xi)\rangle^{-3} \\
& \leq e^{(m+3) L \omega((1-\tau) x+\tau y, \xi)} e^{(m+3) L} C^{3} e^{-3 \frac{n}{j} \varphi^{*}\left(\frac{j}{n}\right)} .
\end{aligned}
$$

Moreover, by (2.18) (where $k \in \mathbb{N}_{0}$ is as in (2.16)) and (0.4)

$$
\begin{aligned}
e^{(m+3) L \omega((1-\tau) x+\tau y, \xi)} & \leq e^{(m+3) L^{k+1} \omega(x, y, \xi)} e^{(m+3) L^{k+1}+\cdots+(m+3) L^{2}} \\
& \leq e^{(m+3) L^{k+2}(\omega(x)+\omega(y)+\omega(\xi))} e^{(m+3) L^{k+2}+\cdots+(m+3) L^{2}} .
\end{aligned}
$$

Take $0<s C_{1} \leq \ell<n$. Since $f, g \in \mathcal{S}_{\omega}\left(\mathbb{R}^{d}\right)$ there exist $C_{\ell}^{\prime \prime}>0$ (depending on $\ell, m, \tau$ ) and $D>0$ (depending on $m, \tau$ ) such that

$$
\begin{aligned}
\left|D_{y}^{\eta_{3}} f(y)\right| & \leq C_{\ell}^{\prime \prime} e^{\ell L^{\widetilde{p}+1} \varphi^{*}\left(\frac{\left|\eta_{3}\right|}{\ell L^{\tilde{p}+1}}\right)} e^{-\left((m+3) L^{k+2}+1\right) \omega(y)} \\
|g(x)| & \leq D e^{-\left((m+3) L^{k+2}+1\right) \omega(x)} .
\end{aligned}
$$


From Lemmas 0.8 and 0.1 , we have by the choice of $\widetilde{p} \in \mathbb{N}$

$$
\begin{aligned}
& \sum_{\eta_{1}+\eta_{2}+\eta_{3}=\eta} \frac{\eta !}{\eta_{1} ! \eta_{2} ! \eta_{3} !}|\tau|^{\left|\eta_{1}+\eta_{2}\right|} e^{n L^{\widetilde{p}+1} \varphi^{*}\left(\frac{\left|\eta_{1}\right|}{n L^{\tilde{p}}+1}\right)} e^{n L^{\widetilde{p}+1} \varphi^{*}\left(\frac{\left|\eta_{2}\right|}{n L^{\tilde{p}}+1}\right)} e^{\ell L^{\tilde{p}+1} \varphi^{*}\left(\frac{\left|\eta_{3}\right|}{\ell L^{\tilde{p}+1}}\right)} \\
& \leq\langle\tau\rangle^{|\eta|} e^{\ell L^{\widetilde{p}+1} \varphi^{*}\left(\frac{|\eta|}{\ell L^{\tilde{p}+1}}\right)} \sum_{\eta_{1}+\eta_{2}+\eta_{3}=\eta} \frac{\eta !}{\eta_{1} ! \eta_{2} ! \eta_{3} !} \\
& \leq e^{\ell L \varphi^{*}\left(\frac{|\eta|}{\ell L}\right)} e^{\ell L \sum_{r=1}^{\widetilde{p}} L^{r}} .
\end{aligned}
$$

By Corollaries 0.23 and 0.20 , there exist $C_{1}, C_{2}, C_{3}>0$ such that

$$
\left|b_{\eta}\right| \leq e^{s C_{1}} e^{-s C_{1} \varphi^{*}\left(\frac{|\eta|}{s C_{1}}\right)}, \quad\left|\frac{1}{G^{s}(\xi)}\right| \leq C_{3}^{s} e^{-s C_{2} \omega(\xi)} .
$$

We then estimate the modulus of (2.20) by

$$
\begin{aligned}
& \int\left(\int C _ { 3 } ^ { s } e ^ { - s C _ { 2 } \omega ( \xi ) } \sum _ { \eta \in \mathbb { N } _ { 0 } ^ { d } } e ^ { s C _ { 1 } } e ^ { - s C _ { 1 } \varphi ^ { * } ( \frac { | \eta | } { s C _ { 1 } } ) } \left(\int \sum_{j=N+1}^{\infty} C_{n} C_{n}^{\prime} e^{\ell L \varphi^{*}\left(\frac{|\eta|}{\ell L}\right)} e^{\ell L \sum_{r=1}^{\widetilde{p}} L^{r}} \times\right.\right. \\
& \times(2 R)^{-\rho j} C^{3} e^{(m+3) L^{k+2}+\cdots+(m+3) L^{2}} e^{(m+3) L} e^{(m+3) L^{k+2}(\omega(x)+\omega(y)+\omega(\xi))} \times \\
& \left.\left.\times e^{-3 \frac{n}{j} \varphi^{*}\left(\frac{j}{n}\right)} C_{\ell}^{\prime \prime} e^{-\left((m+3) L^{k+2}+1\right) \omega(y)} d y\right) d \xi\right) D e^{-\left((m+3) L^{k+2}+1\right) \omega(x)} d x .
\end{aligned}
$$

Since $\ell \geq s C_{1}$, the series depending on $\eta \in \mathbb{N}_{0}^{d}$ converges (as in (1.14)). Notice that the constant depending on $n \in \mathbb{N}$ is $C_{n} C_{n}^{\prime}$. Take $s \in \mathbb{N}_{0}$ such that $s C_{2} \geq(m+3) L^{k+2}+1$. This yields, for $j_{l} \leq N+1<j_{l+1}$, the following estimate for the modulus of $a)$ :

$$
E_{\ell}\left(\int e^{-\omega(x)} d x\right)\left(\int e^{-\omega(y)} d y\right)\left(\int e^{-\omega(\xi)} d \xi\right)\left(\sum_{n=l}^{\infty} \sum_{j=j_{n}}^{j_{n+1}-1} \frac{C_{n} C_{n}^{\prime}}{(2 R)^{\rho j} e^{3 \frac{n}{j} \varphi^{*}\left(\frac{j}{n}\right)}}\right),
$$

where $E_{\ell}>0$ is a constant depending on $\ell$. The convergence of the integrals is guaranteed by property $(\gamma)$ of the weight function. By assumption we have $3 \frac{n}{j} \varphi^{*}\left(\frac{j}{n}\right) \geq \log \left(C_{2 n L^{\tilde{p}+1}}\right)+\log \left(C_{n L^{\tilde{p}+1}}^{\prime}\right)+n$. This proves $\left.a\right)$.

For the integral in $b$ ), we proceed in a similar way: we consider the same integration by parts (1.6) as in the previous case, for some $s \in \mathbb{N}$ determined later. Then, the integrand is equal to

$$
\begin{aligned}
& e^{i(x-y) \cdot \xi} \frac{1}{G^{s}(\xi)} G^{s}\left(D_{y}\right)\left(\Psi_{N+1} \sum_{l=0}^{N} p_{l} \cdot f(y)\right) \\
& =\frac{e^{i(x-y) \cdot \xi}}{G^{s}(\xi)} \sum_{\eta \in \mathbb{N}_{0}^{d}} b_{\eta} \sum_{\eta_{1}+\eta_{2}+\eta_{3}=\eta} \frac{\eta !}{\eta_{1} ! \eta_{2} ! \eta_{3} !} \tau^{\left|\eta_{1}+\eta_{2}\right|} D_{y}^{\eta_{1}} \Psi_{N+1}\left(\sum_{l=0}^{N} D_{y}^{\eta_{2}} p_{l}\right) D_{y}^{\eta_{3}} f(y) .
\end{aligned}
$$


We claim that

$$
\begin{aligned}
& \int\left(\int \frac{1}{G^{s}(\xi)} \sum_{\eta \in \mathbb{N}_{0}^{d}} b_{\eta} \sum_{\eta_{1}+\eta_{2}+\eta_{3}=\eta} \frac{\eta !}{\eta_{1} ! \eta_{2} ! \eta_{3} !} \tau^{\left|\eta_{1}+\eta_{2}\right|} \times\right. \\
& \left.\times\left(\int e^{i(x-y) \cdot \xi} D_{y}^{\eta_{1}} \Psi_{N+1}\left(\sum_{l=0}^{N} D_{y}^{\eta_{2}} p_{l}\right) D_{y}^{\eta_{3}} f(y) d y\right) d \xi\right) g(x) d x
\end{aligned}
$$

converges to zero as $N \rightarrow \infty$. Given $N \in \mathbb{N}$, we take $n \in \mathbb{N}$ such that $j_{n} \leq N+1<j_{n+1}$. If $\Psi_{N+1}((1-\tau) x+\tau y, \xi) \neq 0$, then $\log \left(\frac{\langle((1-\tau) x+\tau y, \xi)\rangle}{2 R}\right) \geq$ $\frac{n}{N+1} \varphi^{*}\left(\frac{N+1}{n}\right)$. Put $0<s C_{1} \leq \ell<n$, where $C_{1}>0$ is the constant from Corollary 0.23 . Similarly as before, for the same constants $C_{n}=C_{2 n L^{\tilde{p}}+1}>0$ and $C_{n}^{\prime}=C_{n L^{\widetilde{p}+1}}>0$ we have this estimate for the modulus of $b$ ):

$$
\begin{aligned}
& \int\left(\int C _ { 3 } ^ { s } e ^ { - s C _ { 2 } \omega ( \xi ) } \sum _ { \eta \in \mathbb { N } _ { 0 } ^ { d } } e ^ { s C _ { 1 } } e ^ { - s C _ { 1 } \varphi ^ { * } ( \frac { | \eta | } { s C _ { 1 } } ) } \left(\int C_{n} C_{n}^{\prime} e^{\ell L \varphi^{*}\left(\frac{|\eta|}{\ell L}\right)} e^{\ell L \sum_{r=1}^{\tilde{p}} L^{r}} \times\right.\right. \\
& \times\left(\sum_{l=0}^{N}(2 R)^{-\rho l}\right) C^{3} e^{(m+3)\left(L^{k+2}+\cdots+L^{2}+L\right)} e^{(m+3) L^{k+2}(\omega(x)+\omega(y)+\omega(\xi))} \times \\
& \left.\left.\times e^{-3 \frac{n}{N+1} \varphi^{*}\left(\frac{N+1}{n}\right)} C_{\ell}^{\prime \prime} e^{-\left((m+3) L^{k+2}+1\right) \omega(y)} d y\right) d \xi\right) D e^{-\left((m+3) L^{k+2}+1\right) \omega(x)} d x .
\end{aligned}
$$

The same $s \in \mathbb{N}$ as before guarantees the convergence of the integrals and of the series on $\eta \in \mathbb{N}_{0}^{d}$. The proof of $\left.b\right)$ now follows by the selection of $\left(j_{n}\right)$ and because the series $\sum_{l=0}^{\infty}(2 R)^{-\rho l}$ is convergent.

\subsection{Properties of formal sums}

Example 2.12. Let $a(x, y, \xi)$ be an amplitude in $\mathrm{GA}_{\rho}^{m, \omega}$ and let

$$
p_{j}(x, \xi):=\left.\sum_{|\beta+\gamma|=j} \frac{1}{\beta ! \gamma !} \tau^{|\beta|}(1-\tau)^{|\gamma|} \partial_{\xi}^{\beta+\gamma}\left(-D_{x}\right)^{\beta} D_{y}^{\gamma} a(x, y, \xi)\right|_{y=x} .
$$

Then, the formal series $\sum p_{j}$ belongs to $\operatorname{FGS}_{\rho}^{\max \{m, m L\}, \omega}$ for all $\tau \in \mathbb{R}$.

Proof. We want to estimate $\left|D_{x}^{\alpha} D_{\xi}^{\epsilon} p_{j}(x, \xi)\right|$ for all $j \in \mathbb{N}_{0}, \alpha, \epsilon \in \mathbb{N}_{0}^{d}$ and $\log \left(\frac{\langle(x, \xi)\rangle}{R}\right) \geq \frac{n}{j} \varphi^{*}\left(\frac{j}{n}\right)$, for some $R \geq 1$. We consider $\widetilde{p} \in \mathbb{N}_{0}$ so that $2 \leq e^{\rho \widetilde{p}}$. Since $a \in \mathrm{GA}_{\rho}^{m, \omega}$, by the chain rule we have that for all $n \in \mathbb{N}$ there exists 


$$
\begin{aligned}
& \left.C_{n}=C_{2 n L^{\widetilde{p}}}>0 \text { such that (for }|\beta+\gamma|=j\right) \\
& \qquad \begin{array}{l}
\left|D_{x}^{\alpha} D_{\xi}^{\epsilon}\left[\left.\partial_{\xi}^{\beta+\gamma}\left(-D_{x}\right)^{\beta} D_{y}^{\gamma} a(x, y, \xi)\right|_{y=x}\right]\right| \\
\quad \leq \sum_{\widetilde{\alpha} \leq \alpha}\left(\begin{array}{c}
\alpha \\
\widetilde{\alpha}
\end{array}\right)\left|D_{x}^{\widetilde{\alpha}} D_{y}^{\alpha-\widetilde{\alpha}} D_{\xi}^{\epsilon}\left[\left.\partial_{\xi}^{\beta+\gamma}\left(-D_{x}\right)^{\beta} D_{y}^{\gamma} a(x, y, \xi)\right|_{y=x}\right]\right| \\
\leq C_{n} e^{m \omega(x, x, \xi)}\langle(x, x, \xi)\rangle^{-\rho(|\alpha+\epsilon|+2 j)} e^{2 n L^{\widetilde{p}} \rho \varphi^{*}\left(\frac{|\alpha+\epsilon|+2 j}{2 n L^{\widetilde{p}}}\right)} \sum_{\widetilde{\alpha} \leq \alpha}\left(\begin{array}{c}
\alpha \\
\widetilde{\alpha}
\end{array}\right) .
\end{array}
\end{aligned}
$$

By formula (0.11), we have $e^{2 n L^{\widetilde{p}} \rho \varphi^{*}\left(\frac{|\alpha+\epsilon|+2 j}{2 n L^{\widetilde{p}}}\right)} \leq e^{n L^{\widetilde{p}} \rho \varphi^{*}\left(\frac{|\alpha+\epsilon|+j}{n L^{\widetilde{p}}}\right)} e^{n \rho \varphi^{*}\left(\frac{j}{n}\right)}$. Furthermore, $\log \left(\frac{\langle(x, \xi)\rangle}{R}\right) \geq \frac{n}{j} \varphi^{*}\left(\frac{j}{n}\right)$ implies $e^{n \rho \varphi^{*}\left(\frac{j}{n}\right)} \leq\langle(x, \xi)\rangle^{\rho j}$. Since $\sum\left(\frac{\alpha}{\alpha}\right)=$ $2^{|\alpha|}$, from the choice of $\tilde{p} \in \mathbb{N}_{0}$, we have, by $(0.10)$,

$$
2^{|\alpha|} e^{n L^{\widetilde{p}} \rho \varphi^{*}\left(\frac{|\alpha+\epsilon|+j}{n L \tilde{p}}\right)} \leq e^{n \rho \varphi^{*}\left(\frac{|\alpha+\epsilon|+j}{n}\right)} e^{n \rho \sum_{s=1}^{\widetilde{p}} L^{s}} .
$$

From $(0.3)$ we have that $\omega(x, \xi) \leq \omega(x, x, \xi) \leq L \omega(x, \xi)+L$. Thus, we obtain

$$
\begin{aligned}
& \left|D_{x}^{\alpha} D_{\xi}^{\epsilon}\left[\left.\partial_{\xi}^{\beta+\gamma}\left(-D_{x}\right)^{\beta} D_{y}^{\gamma} a(x, y, \xi)\right|_{y=x}\right]\right| \\
& \quad \leq C_{n} e^{n \rho \sum_{s=1}^{\widetilde{p}} L^{s}}\langle(x, \xi)\rangle^{-\rho(|\alpha+\epsilon|+j)} e^{n \rho \varphi^{*}\left(\frac{|\alpha+\epsilon|+j}{n}\right)} e^{\max \{0, m L\}} e^{\max \{m, m L\} \omega(x, \xi)} .
\end{aligned}
$$

Finally, by Lemma $0.1(2),(1)$, we have

$$
\begin{aligned}
\sum_{|\beta+\gamma|=j} \frac{|\tau|^{|\beta|}|1-\tau|^{|\gamma|}}{\beta ! \gamma !} & \leq \sum_{|\beta|=0}^{j} \frac{d^{|\beta|}|\tau|^{|\beta|}}{|\beta| !} \sum_{|\gamma|=0}^{j} \frac{d^{|\gamma|}|1-\tau|^{|\gamma|}}{|\gamma| !} \\
& \leq\left(\sum_{k=0}^{j} \frac{(d|\tau|)^{k}}{k !} \sum_{|\beta|=k} 1\right)\left(\sum_{l=0}^{j} \frac{(d|1-\tau|)^{l}}{l !} \sum_{|\gamma|=l} 1\right) \\
& \leq \sum_{k=0}^{j} \frac{\left(d^{2}|\tau|\right)^{k}}{k !} \sum_{l=0}^{j} \frac{\left(d^{2}|1-\tau|\right)^{l}}{l !} \leq e^{d^{2}|\tau|} e^{d^{2}|1-\tau|}
\end{aligned}
$$

Then, we obtain the result.

Proposition 2.13. Let $\sum p_{j} \in \mathrm{FGS}_{\rho}^{m, \omega}$ be a formal sum. Then $\sum q_{j}$ given by

$$
q_{j}(x, \xi):=\sum_{|\alpha|+h=j} \frac{1}{\alpha !}(1-2 \tau)^{|\alpha|} \partial_{\xi}^{\alpha} D_{x}^{\alpha}\left(p_{h}(x,-\xi)\right)
$$

is a formal sum in $\mathrm{FGS}_{\rho}^{m, \omega}$. 
Proof. By assumption, there is $R \geq 1$ such that for all $n \in \mathbb{N}$ there exists $C_{n}=C_{2 n}>0$ satisfying (using (0.11))

$$
\begin{aligned}
\mid D_{x}^{\gamma} D_{\xi}^{\beta} & q_{j}(x, \xi) \mid \\
\leq & \sum_{|\alpha|+h=j} \frac{1}{\alpha !}|1-2 \tau|^{|\alpha|}\left|D_{x}^{\alpha+\gamma} D_{\xi}^{\alpha+\beta} p_{h}(x,-\xi)\right| \\
\leq & C_{n} e^{m \omega(x, \xi)} \sum_{|\alpha|+h=j} \frac{1}{\alpha !}|1-2 \tau|^{|\alpha|}\langle(x, \xi)\rangle^{-\rho(|2 \alpha+\gamma+\beta|+h)} e^{2 n \rho \varphi^{*}\left(\frac{|2 \alpha+\gamma+\beta|+h}{2 n}\right)} \\
\leq & C_{n} e^{m \omega(x, \xi)}\langle(x, \xi)\rangle^{-\rho(|\gamma+\beta|+j)} e^{n \rho \varphi^{*}\left(\frac{|\gamma+\beta|+j}{n}\right)} \times \\
& \times \sum_{|\alpha|+h=j} \frac{1}{\alpha !}|1-2 \tau|^{|\alpha|}\langle(x, \xi)\rangle^{-\rho|\alpha|} e^{n \rho \varphi^{*}\left(\frac{|\alpha|}{n}\right)},
\end{aligned}
$$

for every $j \in \mathbb{N}, \gamma, \beta \in \mathbb{N}_{0}^{d}$, and $\log \left(\frac{\langle(x, \xi)\rangle}{R}\right) \geq \frac{2 n}{j} \varphi^{*}\left(\frac{j}{2 n}\right)$. We take $\log \left(\frac{\langle(x, \xi)\rangle}{R}\right) \geq$ $\frac{n}{j} \varphi^{*}\left(\frac{j}{n}\right)\left(\geq \frac{2 n}{j} \varphi^{*}\left(\frac{j}{2 n}\right)\right)$. In particular, as $|\alpha| \leq j$, we obtain $e^{n \rho \varphi^{*}\left(\frac{|\alpha|}{n}\right)} \leq$ $\langle(x, \xi)\rangle^{\rho|\alpha|}$. Finally, using Lemma 0.1, we have

$$
\sum_{|\alpha|+h=j} \frac{1}{\alpha !}|1-2 \tau|^{|\alpha|} \leq \sum_{|\alpha|=0}^{j} \frac{d^{|\alpha|}|1-2 \tau|^{|\alpha|}}{|\alpha| !} \leq \sum_{k=0}^{j} \frac{(d|1-2 \tau|)^{k}}{k !} \sum_{|\alpha|=k} 1 \leq e^{d^{2}|1-2 \tau|},
$$

which gives the conclusion.

Definition 2.14. Let $\sum p_{j} \in \mathrm{FGS}_{\rho}^{m, \omega}$. We define $\left(\sum p_{j}\right)^{t}$ as the formal sum $\sum q_{j}$ in $\mathrm{FGS}_{\rho}^{m, \omega}$ given in Proposition 2.13.

Proposition 2.15. Let $\sum p_{j} \in \mathrm{FGS}_{\rho}^{m_{1}, \omega}$ and $\sum q_{j} \in \mathrm{FGS}_{\rho}^{m_{2}, \omega}$. The formal sum $\sum r_{j}$ given by

$$
r_{j}(x, \xi):=\sum_{|\beta+\gamma|+k+h=j} \frac{(-1)^{|\beta|}}{\beta ! \gamma !} \tau^{|\beta|}(1-\tau)^{|\gamma|}\left(\partial_{\xi}^{\gamma} D_{x}^{\beta} p_{h}(x, \xi)\right)\left(\partial_{\xi}^{\beta} D_{x}^{\gamma} q_{k}(x, \xi)\right)
$$

belongs to $\mathrm{FGS}_{\rho}^{m_{1}+m_{2}, \omega}$. 
Proof. Let $\tilde{p} \in \mathbb{N}_{0}$ satisfy $2 \leq e^{\rho \widetilde{p}}$. By assumption, there is $R \geq 1$ such that for all $n \in \mathbb{N}$ there exists $C_{n}=C_{2 n L \widetilde{p}}>0$ with

$$
\begin{aligned}
& \left|D_{x}^{\alpha} D_{\xi}^{\epsilon} r_{j}(x, \xi)\right| \\
& \leq \sum_{|\beta+\gamma|+k+h=j} \frac{1}{\beta ! \gamma !}|\tau|^{|\beta|}|1-\tau|^{|\gamma|}\left|D_{x}^{\alpha} D_{\xi}^{\epsilon}\left(\partial_{\xi}^{\gamma} D_{x}^{\beta} p_{h}(x, \xi) \cdot \partial_{\xi}^{\beta} D_{x}^{\gamma} q_{k}(x, \xi)\right)\right| \\
& \leq \sum_{|\beta+\gamma|+k+h=j} \frac{1}{\beta ! \gamma !}|\tau|^{|\beta|} \mid 1-\tau^{|\gamma|} \times \\
& \times \sum_{\widetilde{\alpha} \leq \alpha ; \widetilde{\epsilon} \leq \epsilon}\left(\begin{array}{c}
\alpha \\
\widetilde{\alpha}
\end{array}\right)\left(\begin{array}{c}
\epsilon \\
\widetilde{\epsilon}
\end{array}\right)\left|D_{x}^{\widetilde{\alpha}+\beta} D_{\xi}^{\widetilde{\epsilon}+\gamma} p_{h}(x, \xi)\right|\left|D_{x}^{\alpha-\widetilde{\alpha}+\gamma} D_{\xi}^{\epsilon-\widetilde{\epsilon}+\beta} q_{k}(x, \xi)\right| \\
& \leq C_{n} e^{\left(m_{1}+m_{2}\right) \omega(x, \xi)}\langle(x, \xi)\rangle^{-\rho(|\alpha+\epsilon|+j)} \sum_{|\beta+\gamma|+k+h=j} \frac{|\tau|^{|\beta|}|1-\tau|^{|\gamma|}}{\beta ! \gamma !}\langle(x, \xi)\rangle^{-\rho|\beta+\gamma|} \times \\
& \times \sum_{\widetilde{\alpha} \leq \alpha ; \widetilde{\epsilon} \leq \epsilon}\left(\begin{array}{c}
\alpha \\
\widetilde{\alpha}
\end{array}\right)\left(\begin{array}{l}
\epsilon \\
\widetilde{\epsilon}
\end{array}\right) e^{2 n L^{\widetilde{p}} \rho \varphi^{*}\left(\frac{|\widetilde{\alpha}+\widetilde{\epsilon}+\beta+\gamma|+h}{2 n L^{\widetilde{p}}}\right)} e^{2 n L^{\widetilde{p}} \rho \varphi^{*}\left(\frac{|\alpha-\widetilde{\alpha}+\epsilon-\widetilde{\epsilon}+\beta+\gamma|+k}{2 n L \tilde{p}}\right)}
\end{aligned}
$$

for all $j \in \mathbb{N}_{0}, \alpha, \epsilon \in \mathbb{N}_{0}^{d}$, and $\log \left(\frac{\langle(x, \xi)\rangle}{R}\right) \geq \frac{2 n L^{\widetilde{p}}}{j} \varphi^{*}\left(\frac{j}{2 n L^{\tilde{p}}}\right)$. By Lemma 0.8, we have

$$
e^{2 n L^{\widetilde{p}} \rho \varphi^{*}\left(\frac{|\widetilde{\alpha}+\tilde{\epsilon}+\beta+\gamma|+h}{2 n L^{\widetilde{p}}}\right)} e^{2 n L^{\widetilde{p}} \rho \varphi^{*}\left(\frac{|\alpha-\widetilde{\alpha}+\epsilon-\widetilde{\epsilon}+\beta+\gamma|+k}{2 n L^{\widetilde{p}}}\right)} \leq e^{n L^{\widetilde{p}} \rho \varphi^{*}\left(\frac{|\alpha+\epsilon|+j}{n L^{\tilde{p}}}\right)} e^{n \rho \varphi^{*}\left(\frac{|\beta+\gamma|}{n}\right)} .
$$

Then, as $\sum\left(\begin{array}{c}\alpha \\ \widetilde{\alpha}\end{array}\right)\left(\begin{array}{c}\epsilon \\ \widetilde{\epsilon}\end{array}\right)=2^{|\alpha+\epsilon|}$, it follows by $(0.10)$ and the choice of $\widetilde{p} \in \mathbb{N}_{0}$, that

$$
e^{n L^{\widetilde{p}} \rho \varphi^{*}\left(\frac{|\alpha+\epsilon|+j}{n L \widetilde{p}}\right)} 2^{|\alpha+\epsilon|} \leq e^{n \rho \varphi^{*}\left(\frac{|\alpha+\epsilon|+j}{n}\right)} e^{n \rho \sum_{s=1}^{\widetilde{p}} L^{s}} .
$$

On the other hand, if we take $\log \left(\frac{\langle(x, \xi)\rangle}{R}\right) \geq \frac{n}{j} \varphi^{*}\left(\frac{j}{n}\right)\left(\geq \frac{2 n L^{\widetilde{p}}}{j} \varphi^{*}\left(\frac{j}{2 n L^{\tilde{p}}}\right)\right)$, this implies $\log \left(\frac{\langle(x, \xi)\rangle}{R}\right) \geq \frac{n}{|\beta+\gamma|} \varphi^{*}\left(\frac{|\beta+\gamma|}{n}\right)$ for $|\beta+\gamma|>0$, and therefore as in the proof of Example 2.12 we have

$$
\begin{gathered}
\sum_{|\beta+\gamma|+k+h=j} \frac{1}{\beta ! \gamma !}|\tau|^{|\beta|}|1-\tau|^{|\gamma|}\langle(x, \xi)\rangle^{-\rho|\beta+\gamma|} e^{n \rho \varphi^{*}\left(\frac{|\beta+\gamma|}{n}\right)} \\
\leq\left(\sum_{|\beta|=0}^{j} \frac{|\tau|^{|\beta|}}{\beta !}\right)\left(\sum_{|\gamma|=0}^{j} \frac{|1-\tau|^{|\gamma|}}{\gamma !}\right) \leq e^{d^{2}|\tau|} e^{d^{2}|1-\tau|} .
\end{gathered}
$$

This completes the proof.

Definition 2.16. Let $\sum p_{j} \in \mathrm{FGS}_{\rho}^{m_{1}, \omega}$ and $\sum q_{j} \in \mathrm{FGS}_{\rho}^{m_{2}, \omega}$. We define $\left(\sum p_{j}\right) \circ\left(\sum q_{j}\right)$ as the formal sum $\sum r_{j}$ in $\mathrm{FGS}_{\rho}^{m_{1}+m_{2}, \omega}$ given by Proposition 2.15 . 
The following result is taken from [65, Proposition 2.19]. See for example [47, Proposición 2.2.8] for a detailed proof.

Proposition 2.17. Let $\sum p_{j}$ and $\sum q_{j}$ be formal sums in $\mathrm{FGS}_{\rho}^{m_{1}, \omega}$ and in $\mathrm{FGS}_{\rho}^{m_{2}, \omega}$. If $\sum p_{j} \sim \sum p_{j}^{\prime}$ and $\sum q_{j} \sim \sum q_{j}^{\prime}$ for some formal sums $\sum p_{j}^{\prime}, \sum q_{j}^{\prime}$, then $\left(\sum p_{j}\right) \circ\left(\sum q_{j}\right) \sim\left(\sum p_{j}^{\prime}\right) \circ\left(\sum q_{j}^{\prime}\right)$.

\subsection{Behaviour of the kernel of a pseudodifferential operator}

To study the transposition and composition of operators, we need to analyse the behaviour of the kernel of a pseudodifferential operator. We will show, similarly to the local case, that the kernel satisfies the estimates of a function in $\mathcal{S}_{\omega}\left(\mathbb{R}^{2 d}\right)$, but outside a strip around the diagonal.

For $r>0$, we denote

$$
\Delta_{r}:=\left\{(x, y) \in \mathbb{R}^{2 d}:|x-y|<r\right\} .
$$

We begin with a lemma.

Lemma 2.18. Given $r>0$, there exists $\chi \in C^{\infty}\left(\mathbb{R}^{2 d}\right)$ satisfying $0 \leq \chi \leq 1$, $\chi(x, y)=1$ if $(x, y) \in \mathbb{R}^{2 d} \backslash \Delta_{r}$ and $\chi(x, y)=0$ if $(x, y) \in \Delta_{r / 2}$ such that for all $\lambda>0$ there exists $C_{\lambda}>0$ with

$$
\left|D_{x}^{\alpha} D_{y}^{\beta} \chi(x, y)\right| \leq C_{\lambda} e^{\lambda \varphi^{*}\left(\frac{|\alpha+\beta|}{\lambda}\right)}, \quad \alpha, \beta \in \mathbb{N}_{0}^{d}, x, y \in \mathbb{R}^{d} .
$$

Proof. Let $\psi \in \mathcal{D}_{(\omega)}\left(\mathbb{R}^{d}\right)$ such that $0 \leq \psi \leq 1, \psi(\xi)=1$ if $|\xi|<r / 2$ and $\psi(\xi)=0$ if $|\xi| \geq r$. Set $\phi:=1-\psi$. Then, it is enough to take $\chi(x, y):=$ $\phi(x-y), x, y \in \mathbb{R}^{d}$.

Leibniz rule yields

Lemma 2.19. If $\chi$ is the function in Lemma 2.18, then $\chi f$ and $(1-\chi) f$ belong to $\mathcal{S}_{\omega}\left(\mathbb{R}^{2 d}\right)$ for all $f \in \mathcal{S}_{\omega}\left(\mathbb{R}^{2 d}\right)$.

This result is crucial for the proof of Theorem 2.24. It is an improvement of [55, Theorem 6.3.3] and [58, Proposition 5] (see the introduction to this chapter). See [33, Theorem 2.17] for the corresponding result in the local case. 
Theorem 2.20. Let $a \in \mathrm{GA}_{\rho}^{m, \omega}$ and $r>0$. The formal kernel

$$
K(x, y):=\int_{\mathbb{R}^{d}} e^{i(x-y) \cdot \xi} a(x, y, \xi) d \xi
$$

satisfies

1. $K(x, y) \in C^{\infty}\left(\mathbb{R}^{2 d} \backslash \overline{\Delta_{r}}\right)$.

2. For every $\lambda>0$ there exists $C_{\lambda}>0$ (depending on $r>0$ ) such that

$$
\left|D_{x}^{\alpha} D_{y}^{\gamma} K(x, y)\right| \leq C_{\lambda} e^{\lambda \varphi^{*}\left(\frac{|\alpha+\gamma|}{\lambda}\right)} e^{-\lambda \omega(x, y)}, \quad \alpha, \gamma \in \mathbb{N}_{0}^{d}, \quad(x, y) \in \mathbb{R}^{2 d} \backslash \Delta_{r} .
$$

Proof. Let $\sigma$ be a weight function satisfying $\omega\left(t^{1 / \rho}\right)=O(\sigma(t))$ as $t \rightarrow \infty$. We consider $\Psi \in \mathcal{D}_{(\sigma)}\left(\mathbb{R}^{2 d}\right)$ with $0 \leq \Psi \leq 1, \Psi(t)=1$ if $\langle t\rangle \leq 2$ and $\Psi(t)=0$ if $\langle t\rangle \geq 3$. We denote by $A_{n}$ the operator associated to the kernel

$$
K_{n}(x, y)=\int_{\mathbb{R}^{d}} e^{i(x-y) \cdot \xi} a(x, y, \xi) \Psi\left(2^{-n}(x, \xi)\right) d \xi .
$$

We show that $K_{n} \rightarrow K$ in $\mathcal{S}_{\omega}^{\prime}\left(\mathbb{R}^{2 d}\right)$. By Lemma 1.11 we have that $K_{n} \in \mathcal{S}_{\omega}\left(\mathbb{R}^{2 d}\right)$ for all $n \in \mathbb{N}$. On the other hand, for the pseudodifferential operator associated to $a$, denoted by $A$, we have $\langle K, \varphi \otimes \chi\rangle=\langle A \chi, \varphi\rangle$ for all $\varphi, \chi \in \mathcal{S}_{\omega}\left(\mathbb{R}^{d}\right)$. Furthermore, since $A_{n} \rightarrow A$ in $L\left(\mathcal{S}_{\omega}\left(\mathbb{R}^{d}\right), \mathcal{S}_{\omega}\left(\mathbb{R}^{d}\right)\right)$ by Theorem 1.15 , we have

$$
\langle K, \varphi \otimes \chi\rangle=\lim _{n \rightarrow \infty}\left\langle A_{n} \chi, \varphi\right\rangle=\lim _{n \rightarrow \infty} \iint K_{n}(x, y) \chi(y) \varphi(x) d y d x,
$$

and this shows $K_{n} \rightarrow K$ in $\sigma\left(\mathcal{S}_{\omega}^{\prime}\left(\mathbb{R}^{2 d}\right), \mathcal{S}_{\omega}\left(\mathbb{R}^{2 d}\right)\right)$ since $\mathcal{S}_{\omega}\left(\mathbb{R}^{d}\right) \otimes \mathcal{S}_{\omega}\left(\mathbb{R}^{d}\right)$ is dense in $\mathcal{S}_{\omega}\left(\mathbb{R}^{2 d}\right)$ (Proposition 0.16). The family $\left\{K_{n}\right\}_{n}$ is equicontinuous as $\mathcal{S}_{\omega}\left(\mathbb{R}^{2 d}\right)$ is barrelled, hence the convergence $K_{n} \rightarrow K$ is also for the topology of precompact convergence. Since $\mathcal{S}_{\omega}\left(\mathbb{R}^{2 d}\right)$ is Montel, $K_{n} \rightarrow K$ converges in the bounded sets of $\mathcal{S}_{\omega}\left(\mathbb{R}^{2 d}\right)$.

On the other hand, there exists $c_{0}>0$ such that $|x-y|_{\infty} \geq c_{0}$ for all $(x, y) \in$ $\mathbb{R}^{2 d} \backslash \Delta_{r}$. We assume that given $(x, y) \in \mathbb{R}^{2 d} \backslash \Delta_{r},|x-y|_{\infty}=\left|x_{l}-y_{l}\right|$, for some $1 \leq l \leq d$. We have, by Leibniz rule,

$$
\begin{aligned}
D_{x}^{\alpha} D_{y}^{\gamma} & \left(K_{n}(x, y)-K_{n+1}(x, y)\right) \\
= & \sum_{\substack{\alpha_{1}+\alpha_{2}+\alpha_{3}=\alpha \\
\gamma_{1}+\gamma_{2}=\gamma}} \frac{\alpha !}{\alpha_{1} ! \alpha_{2} ! \alpha_{3} !} \frac{\gamma !}{\gamma_{1} ! \gamma_{2} !}(-1)^{\left|\gamma_{1}\right|} \int_{\mathbb{R}^{d}} e^{i(x-y) \cdot \xi} \xi^{\alpha_{1}+\gamma_{1}} \times \\
& \times D_{x}^{\alpha_{2}} D_{y}^{\gamma_{2}} a(x, y, \xi) D_{x}^{\alpha_{3}}\left(\Psi\left(2^{-n}(x, \xi)\right)-\Psi\left(2^{-(n+1)}(x, \xi)\right)\right) d \xi
\end{aligned}
$$


for all $\alpha, \gamma \in \mathbb{N}_{0}^{d}, x, y \in \mathbb{R}^{d}$. Following [33, Theorem 2.17], we integrate by parts $N$ times, $N \in \mathbb{N}_{0}$, and we obtain

$$
\begin{aligned}
D_{x}^{\alpha} D_{y}^{\gamma} & \left(K_{n}(x, y)-K_{n+1}(x, y)\right) \\
= & \sum_{\substack{\alpha_{1}+\alpha_{2}+\alpha_{3}=\alpha \\
\gamma_{1}+\gamma_{2}=\gamma}} \frac{\alpha !}{\alpha_{1} ! \alpha_{2} ! \alpha_{3} !} \frac{\gamma !}{\gamma_{1} ! \gamma_{2} !} \frac{(-1)^{N+\left|\gamma_{1}\right|}}{\left|x_{l}-y_{l}\right|^{N}} \int_{\mathbb{R}^{d}} e^{i(x-y) \cdot \xi} \times \\
& \times D_{\xi_{l}}^{N}\left(\xi^{\alpha_{1}+\gamma_{1}} D_{x}^{\alpha_{2}} D_{y}^{\gamma_{2}} a(x, y, \xi) D_{x}^{\alpha_{3}}\left(\Psi\left(2^{-n}(x, \xi)\right)-\Psi\left(2^{-(n+1)}(x, \xi)\right)\right) d \xi\right. \\
= & \sum_{\substack{\alpha_{1}+\alpha_{2}+\alpha_{3}=\alpha \\
\gamma_{1}+\gamma_{2}=\gamma}} \frac{\alpha !}{\alpha_{1} ! \alpha_{2} ! \alpha_{3} !} \frac{\gamma !}{\gamma_{1} ! \gamma_{2} !} \frac{(-1)^{N+\left|\gamma_{1}\right|}}{\left|x_{l}-y_{l}\right|^{N}} \sum_{\substack{N_{1}+N_{2}+N_{3}=N \\
N_{1} \leq\left(\alpha_{1}+\gamma_{1}\right) l}} \frac{N !}{N_{1} ! N_{2} ! N_{3} !} \times \\
& \times \frac{\left(\left(\alpha_{1}+\gamma_{1}\right)_{l}\right) !}{\left(\left(\alpha_{1}+\gamma_{1}\right)_{l}-N_{1}\right) !} \int_{\mathbb{R}^{d}} e^{i(x-y) \cdot \xi} \xi^{\alpha_{1}+\gamma_{1}-N_{1} e_{l}} D_{x}^{\alpha_{2}} D_{y}^{\gamma_{2}} D_{\xi_{l}}^{N_{2}} a(x, y, \xi) \times \\
& \times D_{x}^{\alpha_{3}} D_{\xi_{l}}^{N_{3}}\left(\Psi\left(2^{-n}(x, \xi)\right)-\Psi\left(2^{-(n+1)}(x, \xi)\right)\right) d \xi .
\end{aligned}
$$

Now, we integrate by parts via some power of the ultradifferential operator $G^{s}(D)$, where $s \in \mathbb{N}$ will be determined later, with formula (1.7). The integrand above is then equal to

$$
\begin{aligned}
e^{i(x-y) \cdot \xi} & \frac{1}{G^{s}(y-x)} G^{s}\left(D_{\xi}\right)\left\{\xi^{\alpha_{1}+\gamma_{1}-N_{1} e_{l}} D_{x}^{\alpha_{2}} D_{y}^{\gamma_{2}} D_{\xi_{l}}^{N_{2}} a(x, y, \xi) \times\right. \\
\times & \left.D_{x}^{\alpha_{3}} D_{\xi_{l}}^{N_{3}}\left(\Psi\left(2^{-n}(x, \xi)\right)-\Psi\left(2^{-(n+1)}(x, \xi)\right)\right)\right\} \\
= & e^{i(x-y) \cdot \xi} \frac{1}{G^{s}(y-x)} \sum_{\eta \in \mathbb{N}_{0}^{d}} b_{\eta} \sum_{\substack{\eta_{1}+\eta_{2}+\eta_{3}=\eta \\
\eta_{1} \leq \alpha_{1}+\gamma_{1}-N_{1} e_{l}}} \frac{\eta !}{\eta_{1} ! \eta_{2} ! \eta_{3} !} \times \\
& \times \frac{\left(\alpha_{1}+\gamma_{1}-N_{1} e_{l}\right) !}{\left(\alpha_{1}+\gamma_{1}-N_{1} e_{l}-\eta_{1}\right) !} \xi^{\alpha_{1}+\gamma_{1}-N_{1} e_{l}-\eta_{1}} D_{x}^{\alpha_{2}} D_{y}^{\gamma_{2}} D_{\xi}^{N_{2} e_{l}+\eta_{2}} a(x, y, \xi) \times \\
& \times D_{x}^{\alpha_{3}} D_{\xi}^{N_{3} e_{l}+\eta_{3}}\left(\Psi\left(2^{-n}(x, \xi)\right)-\Psi\left(2^{-(n+1)}(x, \xi)\right)\right) .
\end{aligned}
$$


Thus we obtain

$$
\begin{aligned}
& \left|D_{x}^{\alpha} D_{y}^{\gamma}\left(K_{n}(x, y)-K_{n+1}(x, y)\right)\right| \\
& \leq \sum_{\substack{\alpha_{1}+\alpha_{2}+\alpha_{3}=\alpha \\
\gamma_{1}+\gamma_{2}=\gamma}} \frac{\alpha !}{\alpha_{1} ! \alpha_{2} ! \alpha_{3} !} \frac{\gamma !}{\gamma_{1} ! \gamma_{2} !} \frac{1}{\left|x_{l}-y_{l}\right|^{N}} \sum_{\substack{N_{1}+N_{2}+N_{3}=N \\
N_{1} \leq\left(\alpha_{1}+\gamma_{1}\right)_{l}}} \frac{N !}{N_{1} ! N_{2} ! N_{3} !} \times \\
& \times \frac{\left(\left(\alpha_{1}+\gamma_{1}\right)_{l}\right) !}{\left(\left(\alpha_{1}+\gamma_{1}\right)_{l}-N_{1}\right) !} \sum_{\eta \in \mathbb{N}_{0}^{d}}\left|b_{\eta}\right| \sum_{\substack{\eta_{1}+\eta_{2}+\eta_{3}=\eta \\
\eta_{1} \leq \alpha_{1}+\gamma_{1}-N_{1} e_{l}}} \frac{\eta !}{\eta_{1} ! \eta_{2} ! \eta_{3} !} \frac{\left(\alpha_{1}+\gamma_{1}-N_{1} e_{l}\right) !}{\left(\alpha_{1}+\gamma_{1}-N_{1} e_{l}-\eta_{1}\right) !} \times \\
& \times\left|\frac{1}{G^{s}(y-x)}\right| \int_{\mathbb{R}^{d}}\left|\xi^{\alpha_{1}+\gamma_{1}-N_{1} e_{l}-\eta_{1}}\right|\left|D_{x}^{\alpha_{2}} D_{y}^{\gamma_{2}} D_{\xi}^{N_{2} e_{l}+\eta_{2}} a(x, y, \xi)\right| \times \\
& \times\left|D_{x}^{\alpha_{3}} D_{\xi}^{N_{3} e_{l}+\eta_{3}}\left(\Psi\left(2^{-n}(x, \xi)\right)-\Psi\left(2^{-(n+1)}(x, \xi)\right)\right)\right| d \xi .
\end{aligned}
$$

By Corollaries 0.23 and 0.20 , there exist $C_{1}, C_{2}, C_{3}>0$ such that

$$
\left|b_{\eta}\right| \leq e^{s C_{1}} e^{-s C_{1} \varphi^{*}\left(\frac{|\eta|}{s C_{1}}\right)}, \quad\left|\frac{1}{G^{s}(y-x)}\right| \leq C_{3}^{s} e^{-s C_{2} \omega(y-x)} .
$$

We set $A>1$ such that $A^{2}=d+\frac{1}{c_{0}^{2}}$, and we take $\widetilde{p} \in \mathbb{N}$ so that $\max \{\sqrt{2} A, 6\} \leq$ $e^{\rho \widetilde{p}}$. We fix $\lambda>0$, and we take $\mu>\lambda$. Since $a \in \operatorname{GA}_{\rho}^{m, \omega}$ there exists $C_{\mu}=C_{4 \mu L^{2 \tilde{p}+3}}>0$ with

$$
\begin{aligned}
& \left|D_{x}^{\alpha_{2}} D_{y}^{\gamma_{2}} D_{\xi}^{N_{2} e_{l}+\eta_{2}} a(x, y, \xi)\right| \\
& \quad \leq C_{\mu}\left(\frac{\langle x-y\rangle}{\langle(x, y, \xi)\rangle}\right)^{\rho\left(\left|\alpha_{2}+\gamma_{2}+\eta_{2}\right|+N_{2}\right)} e^{4 \mu L^{2 \widetilde{p}+3} \rho \varphi^{*}\left(\frac{\left|\alpha_{2}+\gamma_{2}+\eta_{2}\right|+N_{2}}{4 \mu L^{2 \widetilde{p}+3}}\right)} e^{m \omega(x, y, \xi)},
\end{aligned}
$$

for each $\alpha_{2}, \gamma_{2}, \eta_{2} \in \mathbb{N}_{0}^{d}, N_{2} \in \mathbb{N}_{0}$, and $x, y, \xi \in \mathbb{R}^{d}$. We see that $\Psi\left(2^{-n}(x, \xi)\right)$ $\Psi\left(2^{-(n+1)}(x, \xi)\right)$ is supported in

$$
B_{n}:=\left\{(x, \xi) \in \mathbb{R}^{2 d}: 2^{n} \leq\langle(x, \xi)\rangle \leq 6 \cdot 2^{n}\right\} .
$$

Hence, there exists $C_{\mu}^{\prime}=C_{4 \mu L^{3 \widetilde{p}+3}}^{\prime}>0$ such that, by $(0.10)$ (and recalling the choice of $\widetilde{p} \in \mathbb{N}$ ),

$$
\begin{aligned}
\mid D_{x}^{\alpha_{3}} & D_{\xi}^{N_{3} e_{l}+\eta_{3}}\left(\Psi\left(2^{-n}(x, \xi)\right)-\Psi\left(2^{-(n+1)}(x, \xi)\right)\right) \mid \\
& \leq 2 C_{\mu}^{\prime} e^{4 \mu L^{3 \widetilde{p}+3} \rho \varphi^{*}\left(\frac{\left|\alpha_{3}+\eta_{3}\right|+N_{3}}{4 \mu L^{3 \widetilde{p}+3}}\right)}\left(\frac{1}{2^{n}}\right)^{\left|\alpha_{3}+\eta_{3}\right|+N_{3}} \\
& \leq 2 C_{\mu}^{\prime} e^{4 \mu L^{3 \widetilde{p}+3} \rho \varphi^{*}\left(\frac{\left|\alpha_{3}+\eta_{3}\right|+N_{3}}{4 \mu L^{3 \tilde{p}+3}}\right)} 6^{\left|\alpha_{3}+\eta_{3}\right|+N_{3}}\langle(x, \xi)\rangle^{-\left|\alpha_{3}+\eta_{3}\right|-N_{3}} \\
& \leq 2 C_{\mu}^{\prime} e^{4 \mu L^{2 \widetilde{p}+3} \rho \sum_{t=1}^{\widetilde{p}} L^{t}} e^{4 \mu L^{2 \widetilde{p}+3} \rho \varphi^{*}\left(\frac{\left|\alpha_{3}+\eta_{3}\right|+N_{3}}{4 \mu L^{2 \tilde{p}+3}}\right)}\langle(x, \xi)\rangle^{-\rho N_{3}}
\end{aligned}
$$


for all $(x, \xi) \in B_{n}$. Now $(0.7)$ yields

$$
|\xi|^{\left|\alpha_{1}+\gamma_{1}\right|-N_{1}-\left|\eta_{1}\right|} \leq\langle(x, \xi)\rangle^{\left|\alpha_{1}+\gamma_{1}\right|-N_{1}} \leq e^{\lambda L^{4} \varphi^{*}\left(\frac{\left|\alpha_{1}+\gamma_{1}\right|}{\lambda L^{4}}\right)} e^{\lambda L^{4} \omega(\langle(x, \xi)\rangle)}\langle(x, \xi)\rangle^{-\rho N_{1}} .
$$

Since $\left|x_{l}-y_{l}\right| \geq c_{0}$, we have

$$
\langle x-y\rangle^{2} \leq 1+d\left|x_{l}-y_{l}\right|^{2} \leq \frac{1}{c_{0}^{2}}\left|x_{l}-y_{l}\right|^{2}+d\left|x_{l}-y_{l}\right|^{2}=A^{2}\left|x_{l}-y_{l}\right|^{2} .
$$

So $\langle x-y\rangle^{N} \leq A^{N}\left|x_{l}-y_{l}\right|^{N}, N \in \mathbb{N}$. Therefore, by Lemma 1.4,

$$
\begin{aligned}
\left(\frac{\langle x-y\rangle}{\langle(x, y, \xi)\rangle}\right)^{\rho\left(\left|\alpha_{2}+\gamma_{2}+\eta_{2}\right|+N_{2}\right)} \frac{1}{\left|x_{l}-y_{l}\right|^{N}} & \leq \sqrt{2}^{\left|\alpha_{2}+\gamma_{2}+\eta_{2}\right|}\left(\frac{\langle x-y\rangle}{\langle(x, y, \xi)\rangle}\right)^{\rho N_{2}} \frac{1}{\left|x_{l}-y_{l}\right|^{N}} \\
& \leq \sqrt{2}^{\left|\alpha_{2}+\gamma_{2}+\eta_{2}\right|}\langle(x, \xi)\rangle^{-\rho N_{2}} \frac{\langle x-y\rangle^{N}}{\left|x_{l}-y_{l}\right|^{N}} \\
& \leq(\sqrt{2} A)^{\left|\alpha_{2}+\gamma_{2}+\eta_{2}\right|+N}\langle(x, \xi)\rangle^{-\rho N_{2}} .
\end{aligned}
$$

Let $C>0$ be the constant in Lemma 0.10(2). By formula (0.12) and using Lemma $0.10(2)$ we obtain, for some $C_{\mu}^{\prime \prime}>0$,

$$
\begin{aligned}
& \frac{\left(\alpha_{1}+\gamma_{1}-N_{1} e_{l}\right) !}{\left(\alpha_{1}+\gamma_{1}-N_{1} e_{l}-\eta_{1}\right) !} \frac{\left(\left(\alpha_{1}+\gamma_{1}\right)_{l}\right) !}{\left(\left(\alpha_{1}+\gamma_{1}\right)_{l}-N_{1}\right) !} \\
& \leq 2^{\left|\alpha_{1}+\gamma_{1}\right|-N_{1}} 2^{\left(\alpha_{1}+\gamma_{1}\right)_{l}} \eta_{1} ! N_{1} \text { ! } \\
& \leq 4^{\left|\alpha_{1}+\gamma_{1}\right|} C_{\mu}^{\prime \prime} e^{\mu L^{3} \varphi^{*}\left(\frac{\left|\eta_{1}\right|}{\mu L^{3}}\right)} e^{\mu L^{2 \tilde{p}} C \varphi_{\sigma}^{*}\left(\frac{N_{1}}{\mu L^{2 \tilde{p}} C}\right)} \\
& \leq 4^{\left|\alpha_{1}+\gamma_{1}\right|} C_{\mu}^{\prime \prime} e^{\mu L^{2 \widetilde{p}} C} e^{\mu L^{3} \varphi^{*}\left(\frac{\left|\eta_{1}\right|}{\mu L^{3}}\right)} e^{\mu L^{2 \widetilde{p}} \rho \varphi^{*}\left(\frac{N_{1}}{\mu L^{2 \tilde{p}}}\right)} \text {. }
\end{aligned}
$$

Writing $\left|\alpha_{2}+\gamma_{2}+\eta_{2}\right|+N=N_{1}+\left(\left|\alpha_{2}+\gamma_{2}+\eta_{2}\right|+N_{2}\right)+N_{3}$, we use Lemma 0.8 (as $\mu>\lambda$ ) as follows:

$$
\begin{aligned}
& 4^{\left|\alpha_{1}+\gamma_{1}\right|} e^{\lambda L^{4} \varphi^{*}\left(\frac{\left|\alpha_{1}+\gamma_{1}\right|}{\lambda L^{4}}\right)} e^{\mu L^{3} \varphi^{*}\left(\frac{\left|\eta_{1}\right|}{\mu L^{3}}\right)}(\sqrt{2} A)^{\left|\alpha_{2}+\gamma_{2}+\eta_{2}\right|+N} e^{\mu L^{2 \widetilde{p}} \rho \varphi^{*}\left(\frac{N_{1}}{\mu L^{2 \widetilde{p}}}\right)} \times \\
& \times e^{4 \mu L^{2 \tilde{p}+3} \rho \varphi^{*}\left(\frac{\left|\alpha_{2}+\gamma_{2}+\eta_{2}\right|+N_{2}}{4 \mu L^{2 \tilde{p}+3}}\right)} e^{4 \mu L^{2 \tilde{p}+3} \rho \varphi^{*}\left(\frac{\left|\alpha_{3}+\eta_{3}\right|+N_{3}}{4 \mu L^{2 \tilde{p}+3}}\right)} \\
& \leq e^{\lambda L^{3}+\lambda L^{4}} e^{\lambda L^{2} \varphi^{*}\left(\frac{\left|\alpha_{1}+\gamma_{1}\right|}{\lambda L^{2}}\right)} e^{\mu L^{3} \varphi^{*}\left(\frac{\left|\eta_{1}\right|}{\mu L^{3}}\right)} e^{\mu L^{\tilde{p}} \rho \sum_{t=1}^{\tilde{p}} L^{\tilde{p}}}\left(e^{4 \mu L^{\tilde{p}+3} \rho \sum_{t=1}^{\tilde{p}} L^{\tilde{p}}}\right)^{2} \times \\
& \times e^{\mu L^{\tilde{p}} \rho \varphi^{*}\left(\frac{N_{1}}{\mu L^{\tilde{p}}}\right)} e^{4 \mu L^{\tilde{p}+3} \rho \varphi^{*}\left(\frac{\left|\alpha_{2}+\gamma_{2}+\eta_{2}\right|+N_{2}}{4 \mu L^{\tilde{p}+3}}\right)} e^{4 \mu L^{\tilde{p}+3} \rho \varphi^{*}\left(\frac{\left|\alpha_{3}+\eta_{3}\right|+N_{3}}{4 \mu L^{\tilde{p}}+3}\right)} \\
& \leq e^{\lambda L^{3}+\lambda L^{4}} e^{\left(8 L^{3}+1\right) \mu L^{\widetilde{p}} \rho \sum_{t=1}^{\widetilde{p}} L^{t}} e^{\lambda L^{2} \varphi^{*}\left(\frac{|\alpha+\gamma|}{\lambda L^{2}}\right)} e^{\mu L^{3} \varphi^{*}\left(\frac{|\eta|}{\mu L^{3}}\right)} e^{\mu L^{\widetilde{p}} \rho \varphi^{*}\left(\frac{N}{\mu L^{\tilde{p}}}\right)} .
\end{aligned}
$$


Then, there exists

$$
C_{\lambda, \mu, s}=e^{s C_{1}} C_{3}^{s} C_{\mu} 2 C_{\mu}^{\prime} e^{4 \mu L^{2 \widetilde{p}+3} \rho \sum_{t=1}^{\widetilde{p}} L^{t}} C_{\mu}^{\prime \prime} e^{\mu L^{2 \widetilde{p}} C} e^{\left(8 L^{3}+1\right) \mu L^{\widetilde{p}} \rho \sum_{t=1}^{\widetilde{p}} L^{t}} e^{\lambda L^{3}+\lambda L^{4}}>0
$$

such that, by Lemma 0.1 ,

$$
\begin{aligned}
\mid D_{x}^{\alpha} D_{y}^{\gamma} & \left.K_{n}(x, y)-K_{n+1}(x, y)\right) \mid \\
\leq & C_{\lambda, \mu, s} 3^{|\alpha|} 2^{|\gamma|} e^{\lambda L^{2} \varphi^{*}\left(\frac{|\alpha+\gamma|}{\lambda L^{2}}\right)}\left(\sum_{\eta \in \mathbb{N}_{0}^{d}} e^{-s C_{1} \varphi^{*}\left(\frac{|\eta|}{s C_{1}}\right)} e^{\mu L^{3} \varphi^{*}\left(\frac{|\eta|}{\mu L^{3}}\right)} 3^{|\eta|}\right) \times \\
& \times e^{-s C_{2} \omega(y-x)} \int_{\mathbb{R}^{d}} e^{\lambda L^{4} \omega(\langle(x, \xi)\rangle)} e^{m \omega(x, y, \xi)}\langle(x, \xi)\rangle^{-\rho N} 3^{N} e^{\mu L^{\widetilde{p}} \rho \varphi^{*}\left(\frac{N}{\mu L^{\widetilde{p}}}\right)} d \xi
\end{aligned}
$$

for all $N \in \mathbb{N}_{0}$. So from (0.8) we obtain using (0.10) and (0.7) that there exists $C^{\prime}>0$ with $\left(\right.$ since $\left.(x, \xi) \in B_{n}\right)$

$$
\begin{aligned}
\inf _{N \in \mathbb{N}_{0}}\langle(x, \xi)\rangle^{-\rho N} 3^{N} e^{\mu L^{\widetilde{p}} \rho \varphi^{*}\left(\frac{N}{\mu L^{\widetilde{p}}}\right)} & \leq e^{\mu \rho \sum_{t=1}^{\widetilde{p}} L^{t}} \inf _{N \in \mathbb{N}_{0}}\langle(x, \xi)\rangle^{-\rho N} e^{\mu \rho \varphi^{*}\left(\frac{N}{\mu}\right)} \\
& \leq e^{\mu \rho \sum_{t=1}^{\widetilde{p}} L^{t}} e^{-\mu \rho \omega(\langle(x, \xi)\rangle)+\rho \log (\langle(x, \xi)\rangle)} \\
& \leq C^{\prime} e^{\mu \rho \sum_{t=1}^{\widetilde{p}} L^{t}} e^{-(\mu-1) \rho \omega(\langle(x, \xi)\rangle)} \\
& \leq C^{\prime} e^{\mu \rho \sum_{t=1}^{\widetilde{p}} L^{t}} e^{-(\mu-2) \rho \omega(\langle(x, \xi)\rangle)} e^{-\rho \omega\left(2^{n}\right)}
\end{aligned}
$$

We put $s \in \mathbb{N}$ such that

$$
s C_{2} \geq(\lambda+m) L^{2}
$$

Then by (0.1) we have

$$
e^{-s C_{2} \omega(y-x)} \leq e^{s C_{2} \omega(x)} e^{-(\lambda+m) L \omega(y)} e^{s C_{2}} .
$$

From $(0.4), \omega(x, y, \xi) \leq L \omega(\langle(x, \xi)\rangle)+L \omega(y)+L$. We take $\mu \geq s C_{1}$ large enough satisfying

$$
(\mu-2) \rho \geq \lambda L^{4}+m L+2 \lambda L+s C_{2} .
$$

Therefore, the series depending on $\eta \in \mathbb{N}_{0}^{d}$ in $(2.21)$ converges by $(0.10)$, proceeding as in (1.14), and we see that

$$
e^{\left(\lambda L^{4}+m L-(\mu-2) \rho\right) \omega(\langle(x, \xi)\rangle)} e^{s C_{2} \omega(x)} \leq e^{-2 \lambda L \omega(\langle(x, \xi)\rangle)} \leq e^{-\lambda L \omega(x)} e^{-\lambda L \omega(\xi)} .
$$

The integral in (2.21) then converges. On the other hand, from (0.10) we have that

$$
3^{|\alpha|} 2^{|\gamma|} e^{\lambda L^{2} \varphi^{*}\left(\frac{|\alpha+\gamma|}{\lambda L^{2}}\right)} \leq e^{\lambda \varphi^{*}\left(\frac{|\alpha+\gamma|}{\lambda}\right)} e^{\lambda L+\lambda L^{2}}
$$


By $(0.3),-\lambda L \omega(x)-\lambda L \omega(y) \leq-\lambda \omega(x, y)+\lambda L$, hence we get that for all $\lambda>0$ there exists $C_{\lambda}>0$ such that

$$
\left|D_{x}^{\alpha} D_{y}^{\gamma}\left(K_{n}(x, y)-K_{n+1}(x, y)\right)\right| \leq C_{\lambda} e^{\lambda \varphi^{*}\left(\frac{|\alpha+\gamma|}{\lambda}\right)} e^{-\lambda \omega(x, y)} e^{-\rho \omega\left(2^{n}\right)}
$$

for all $\alpha, \gamma \in \mathbb{N}_{0}^{d},(x, y) \notin \Delta_{r}$.

By Lemma 2.18, take $\chi$ such that $\chi \equiv 0$ in $\overline{\Delta_{r}}$ and $\chi \equiv 1$ in $\mathbb{R}^{2 d} \backslash \Delta_{2 r}$. Since $\left\{\chi K_{n}\right\}$ is a Cauchy sequence in $\mathcal{S}_{\omega}\left(\mathbb{R}^{2 d}\right)$, there exists $T \in \mathcal{S}_{\omega}\left(\mathbb{R}^{2 d}\right)$ such that $\chi K_{n} \rightarrow T$ in $\mathcal{S}_{\omega}\left(\mathbb{R}^{2 d}\right)$. We notice that $T=\chi K$ in $\mathcal{S}_{\omega}^{\prime}\left(\mathbb{R}^{2 d}\right)$, since $K_{n} \rightarrow K$ in $\mathcal{S}_{\omega}^{\prime}\left(\mathbb{R}^{2 d}\right)$. Therefore we have that for all $\lambda>0$ there exists $C_{\lambda}^{\prime}>0$ such that

$$
\left|D_{x}^{\alpha} D_{y}^{\gamma} K(x, y)\right|=\left|D_{x}^{\alpha} D_{y}^{\gamma} T(x, y)\right| \leq C_{\lambda}^{\prime} e^{\lambda \varphi^{*}\left(\frac{|\alpha+\gamma|}{\lambda}\right)} e^{-\lambda \omega(x, y)},
$$

for all $\alpha, \gamma \in \mathbb{N}_{0}^{d},(x, y) \notin \Delta_{2 r}$. This completes the proof.

We observe that the constant $C_{\lambda}^{\prime}>0$ in (2.22) grows as $r \rightarrow 0$. Indeed, if $r$ tends to zero, then $c_{0}$ tends to zero, too. Therefore, the constant $A>1$ tends to infinity, and also the constant $\widetilde{p} \in \mathbb{N}$. Hence $C_{\lambda, \mu, s}>0$ grows, and so the constant in (2.22).

Now, we prove that an operator given by an amplitude can be decomposed as the sum of (any quantization) of an operator given by a global symbol and an $\omega$-regularizing operator. But first, we need some preparation. The following result is proved in [33, Lemma 3.11].

Lemma 2.21. Let $m \geq n$ and $\frac{1}{e} e^{\frac{m}{j} \varphi^{*}\left(\frac{j}{m}\right)} \leq t \leq e^{\frac{n}{j} \varphi^{*}\left(\frac{j}{n}\right)}$ for $t>0$. Then

$$
e^{n \varphi^{*}\left(\frac{j}{n}\right)} \geq e^{(n-1) \omega(t)} e^{2 n \varphi^{*}\left(\frac{j}{2 n}\right)},
$$

for $j$ large enough.

Lemma 2.22. Let $\tau \in \mathbb{R}$ and let $k \in \mathbb{N}_{0}$ as in (2.16). Then,

$$
\omega(x, y) \leq L^{2} \omega((1-\tau) x+\tau y)+L^{k+2} \omega(y-x)+\sum_{t=1}^{k+2} L^{t}, \quad x, y \in \mathbb{R}^{d} .
$$

Proof. We denote $v=(1-\tau) x+\tau y$ and $w=x-y$. By the triangular inequality, $|x| \leq|v|+|\tau||w|$ and $|y| \leq|v|+|1-\tau||w|$. Then, as $|(x, y)| \leq \sqrt{2} \max \{|x|,|y|\}$, 
by formula (0.1) we obtain

$$
\begin{aligned}
\omega(x, y) & \leq \omega(\sqrt{2}(|v|+(|1-\tau|+|\tau|)|w|)) \\
& \leq L \omega(|v|+(|1-\tau|+|\tau|)|w|)+L \\
& \leq L^{2} \omega(v)+L^{2} \omega\left(2^{k}|w|\right)+L^{2}+L \\
& \leq L^{2} \omega(v)+L^{k+2} \omega(w)+\sum_{t=1}^{k+2} L^{t} .
\end{aligned}
$$

Lemma 2.23. For all $\tau \in \mathbb{R}$ there exists $C=2 \max \left\{(1-\tau)^{2}, \tau^{2}\right\} \geq 1 / 2$ such that

$$
|v|^{2} \leq C\left(|v+t \tau w|^{2}+|v-t(1-\tau) w|^{2}\right), \quad v, w \in \mathbb{R}^{d}, t \in \mathbb{R}
$$

Proof. If $x=v+t \tau w$ and $y=v-t(1-\tau) w$, obviously $v=(1-\tau) x+\tau y$. So, we need to find $C>0$ such that

$$
|(1-\tau) x+\tau y|^{2} \leq C\left(|x|^{2}+|y|^{2}\right) .
$$

Since $2|\tau||1-\tau||x||y| \leq(1-\tau)^{2}|x|^{2}+\tau^{2}|y|^{2}$, we have

$$
\begin{aligned}
|(1-\tau) x+\tau y|^{2} & \leq(1-\tau)^{2}|x|^{2}+\tau^{2}|y|^{2}+2|1-\tau||\tau||x||y| \\
& \leq 2(1-\tau)^{2}|x|^{2}+2 \tau^{2}|y|^{2} \\
& \leq 2 \max \left\{(1-\tau)^{2}, \tau^{2}\right\}\left(|x|^{2}+|y|^{2}\right) .
\end{aligned}
$$

Theorem 2.24. Let $a(x, y, \xi)$ be an amplitude in $\mathrm{GA}_{\rho}^{m, \omega}$ with associated pseudodifferential operator $A$. Then, for any $\tau \in \mathbb{R}$, we can write $A$ as

$$
A=P+R
$$

where $R$ is an $\omega$-regularizing operator and $P$ is the pseudodifferential operator given by

$$
P u(x)=\iint e^{i(x-y) \cdot \xi} p((1-\tau) x+\tau y, \xi) u(y) d y d \xi, \quad u \in \mathcal{S}_{\omega}\left(\mathbb{R}^{d}\right),
$$

being $p \in \operatorname{GS}_{\rho}^{\max \{m, m L\}, \omega}$. Moreover, we have

$$
\left.p(x, \xi) \sim \sum_{j=0}^{\infty} \sum_{|\beta+\gamma|=j} \frac{1}{\beta ! \gamma !} \tau^{|\beta|}(1-\tau)^{|\gamma|} \partial_{\xi}^{\beta+\gamma}\left(-D_{x}\right)^{\beta} D_{y}^{\gamma} a(x, y, \xi)\right|_{y=x} .
$$


Proof. We consider the sequence $\left(j_{n}\right)_{n}$ as in the proof of Theorem 2.6, with the extra assumption (see Third step below):

$$
\frac{n}{j} \varphi^{*}\left(\frac{j}{n}\right) \geq \max \left\{n, \log \left(C_{4 n L^{\tilde{p}+3}}\right), \log \left(D_{4 n L^{\tilde{p}+3}}\right)\right\} \text { for } j_{n} \leq j<j_{n+1},
$$

where $\left(C_{n}\right)_{n}$ and $\left(D_{n}\right)_{n}$ are the sequences of constants of Definition 1.3 and Corollary 2.9, and $\widetilde{p} \in \mathbb{N}_{0}$ is such that $\max \{|1-\tau|, 2|\tau|\} \leq e^{\widetilde{p}}$.

Put

$$
p_{j}(x, \xi):=\left.\sum_{|\beta+\gamma|=j} \frac{1}{\beta ! \gamma !} \tau^{|\beta|}(1-\tau)^{|\gamma|} \partial_{\xi}^{\beta+\gamma}\left(-D_{x}\right)^{\beta} D_{y}^{\gamma} a(x, y, \xi)\right|_{y=x} .
$$

By Example 2.12, $\sum p_{j} \in \mathrm{FGS}_{\rho}^{\max \{m, m L\}, \omega}$. Now, we write

$$
p(x, \xi):=\sum_{j=0}^{\infty} \Psi_{j}(x, \xi) p_{j}(x, \xi),
$$

where $\left(\Psi_{j}\right)_{j}$ is the sequence in (2.15). By Theorem 2.6, we have that $p(x, \xi) \in$ $\mathrm{GS}_{\rho}^{\max \{m, m L\}, \omega}$ and $p \sim \sum p_{j}$. By Lemma 2.8 and Theorem 1.15, the operator $P$ as in the statement of the theorem is continuous. Moreover, by Lemma 2.11, it is the limit of $S_{N, \tau}$ in $L\left(\mathcal{S}_{\omega}\left(\mathbb{R}^{d}\right), \mathcal{S}_{\omega}^{\prime}\left(\mathbb{R}^{d}\right)\right)$, where $S_{N, \tau}$ is the pseudodifferential operator with amplitude $\sum_{j=0}^{N}\left(\Psi_{j}-\Psi_{j+1}\right)((1-\tau) x+\tau y, \xi)\left(\sum_{l=0}^{j} p_{l}((1-\tau) x+\right.$ $\tau y, \xi))$ in $\mathrm{GA}_{\rho}^{\max \left\{0, m^{\prime} L\right\}, \omega}, m^{\prime}$ as in $(2.17)$. That is, for $u \in \mathcal{S}_{\omega}\left(\mathbb{R}^{d}\right)$, we have

$$
\begin{aligned}
P u(x)= & \lim _{N \rightarrow \infty} \iint e^{i(x-y) \cdot \xi}\left(\sum_{j=0}^{N}\left(\Psi_{j}-\Psi_{j+1}\right)((1-\tau) x+\tau y, \xi) \times\right. \\
& \left.\times \sum_{l=0}^{j} p_{l}((1-\tau) x+\tau y, \xi)\right) u(y) d y d \xi .
\end{aligned}
$$

On the other hand, from Lemma 2.10, $A=\sum_{N=0}^{\infty} A_{N}$, where $A_{N}$ is the pseudodifferential operator with amplitude $\left(\Psi_{N}-\Psi_{N+1}\right)((1-\tau) x+\tau y, \xi) a(x, y, \xi)$ in $\mathrm{GA}_{\rho}^{m, \omega} \subseteq \mathrm{GA}_{\rho}^{\max \left\{0, m^{\prime} L\right\}, \omega}$. That is, for $u \in \mathcal{S}_{\omega}\left(\mathbb{R}^{d}\right)$,

$$
\begin{aligned}
A u(x)= & \mathcal{S}_{\omega}\left(\mathbb{R}^{d}\right)-\sum_{N=0}^{\infty} \iint e^{i(x-y) \cdot \xi}\left(\Psi_{N}-\Psi_{N+1}\right)((1-\tau) x+\tau y, \xi) \times \\
& \times a(x, y, \xi) u(y) d y d \xi .
\end{aligned}
$$


Hence, $A-P$ is written as the series $\sum_{N=0}^{\infty} P_{N, \tau}$, where each $P_{N, \tau}$ corresponds to the pseudodifferential operator associated to the amplitude, which belongs to $\mathrm{GA}_{\rho}^{\max \left\{0, m^{\prime} L\right\}, \omega}$, given by

$a_{N, \tau}(x, y, \xi)=\left(\Psi_{N}-\Psi_{N+1}\right)((1-\tau) x+\tau y, \xi)\left(a(x, y, \xi)-\sum_{j=0}^{N} p_{j}((1-\tau) x+\tau y, \xi)\right)$

Our purpose is to show that the kernel $K$ of $A-P$ given by

$$
K(x, y):=\sum_{N=0}^{\infty} K_{N}(x, y)=\sum_{N=0}^{\infty} \int_{\mathbb{R}^{d}} e^{i(x-y) \cdot \xi} a_{N, \tau}(x, y, \xi) d \xi
$$

belongs to $\mathcal{S}_{\omega}\left(\mathbb{R}^{2 d}\right)$. To this, take $r>0$ and let $\chi(x, y)$ be as in Lemma 2.18 satisfying $\chi \equiv 1$ for $(x, y) \in \mathbb{R}^{2 d} \backslash \Delta_{2 r}$ and $\chi \equiv 0$ if $(x, y) \in \overline{\Delta_{r}}$. As $a_{N, \tau} \in$ $\mathrm{GA}_{\rho}^{\max \left\{0, m^{\prime} L\right\}, \omega}$, we have that $K$ satisfies the estimate in Theorem 2.20, and by Lemma 2.19 it follows that $\chi K \in \mathcal{S}_{\omega}\left(\mathbb{R}^{2 d}\right)$. Thus, we want to show that $(1-\chi) K \in \mathcal{S}_{\omega}\left(\mathbb{R}^{2 d}\right)$.

Now, we follow the lines of [64, Theorem 23.2] (see also the scheme of the proof of [33, Theorem 3.13]). Given $x, y \in \mathbb{R}^{d}$, we consider

$$
v=(1-\tau) x+\tau y ; \quad w=x-y .
$$

We proceed similarly as in [33, Theorem 3.13]. The Taylor series of $a(x, y, \xi)=$ $a(v+\tau w, v-(1-\tau) w, \xi)$ at $w=0$ is, for $N \geq 1$,

$$
\begin{array}{r}
a(x, y, \xi)=\sum_{|\beta+\gamma|=0}^{N} \frac{(-1)^{|\gamma|}}{\beta ! \gamma !} \tau^{|\beta|}(1-\tau)^{|\gamma|}(x-y)^{\beta+\gamma}\left(\partial_{x}^{\beta} \partial_{y}^{\gamma} a\right)(v, v, \xi)+ \\
+\sum_{|\beta+\gamma|=N+1} \frac{(-1)^{|\gamma|}}{\beta ! \gamma !} \tau^{|\beta|}(1-\tau)^{|\gamma|} \omega_{\beta \gamma}(x, y, \xi)(x-y)^{\beta+\gamma}
\end{array}
$$

where

$$
\omega_{\beta \gamma}(x, y, \xi):=(N+1) \int_{0}^{1}\left(\partial_{x}^{\beta} \partial_{y}^{\gamma} a\right)(v+t \tau w, v-(1-\tau) t w, \xi)(1-t)^{N} d t .
$$

Note that the expression $\left(\partial_{x}^{\beta} \partial_{y}^{\gamma} a\right)(v, v, \xi)$ means that in $\partial_{x}^{\beta} \partial_{y}^{\gamma} a(x, y, \xi)$, it is necessary to take $v=(1-\tau) x+\tau y$ instead of $x$ and $y$. Then, for $N \geq 1$, we 
have

$$
\begin{aligned}
\int e^{i(x-y) \cdot \xi}\left(\Psi_{N}-\Psi_{N+1}\right)(v, \xi) a(x, y, \xi) d \xi \\
=\int e^{i(x-y) \cdot \xi}\left(\Psi_{N}-\Psi_{N+1}\right)(v, \xi) \times \\
\quad \times\left(\sum_{|\beta+\gamma|=0}^{N} \frac{(-1)^{|\gamma|}}{\beta ! \gamma !} \tau^{|\beta|}(1-\tau)^{|\gamma|}(x-y)^{\beta+\gamma}\left(\partial_{x}^{\beta} \partial_{y}^{\gamma} a\right)(v, v, \xi)\right) d \xi+ \\
\quad+\int e^{i(x-y) \cdot \xi}\left(\Psi_{N}-\Psi_{N+1}\right)(v, \xi) \times \\
\quad \times\left(\sum_{|\beta+\gamma|=N+1} \frac{(-1)^{|\gamma|}}{\beta ! \gamma !} \tau^{|\beta|}(1-\tau)^{|\gamma|} \omega_{\beta \gamma}(x, y, \xi)(x-y)^{\beta+\gamma}\right) d \xi
\end{aligned}
$$

Since $(x-y)^{\beta+\gamma} e^{i(x-y) \cdot \xi}=D_{\xi}^{\beta+\gamma} e^{i(x-y) \cdot \xi}$, we integrate by parts to obtain

$$
\begin{aligned}
\int e^{i(x-y) \cdot \xi}\left(\Psi_{N}-\Psi_{N+1}\right)(v, \xi) a(x, y, \xi) d \xi \\
=\int e^{i(x-y) \cdot \xi}\left(\Psi_{N}-\Psi_{N+1}\right)(v, \xi) a(v, v, \xi) d \xi+ \\
\quad+\sum_{|\beta+\gamma|=1}^{N} \sum_{\alpha \leq \beta+\gamma} \frac{(\beta+\gamma) !}{\alpha !(\beta+\gamma-\alpha) !} \frac{1}{\beta ! \gamma !} \int e^{i(x-y) \cdot \xi} \tau^{|\beta|}(1-\tau)^{|\gamma|}(-1)^{|\gamma|} \times \\
\quad \times D_{\xi}^{\alpha}\left(\Psi_{N}-\Psi_{N+1}\right)(v, \xi)\left(\partial_{x}^{\beta} \partial_{y}^{\gamma} D_{\xi}^{\beta+\gamma-\alpha} a\right)(v, v, \xi) d \xi+ \\
\quad+\sum_{|\beta+\gamma|=N+1} \sum_{\alpha \leq \beta+\gamma} \frac{(\beta+\gamma) !}{\alpha !(\beta+\gamma-\alpha) !} \frac{1}{\beta ! \gamma !} \int e^{i(x-y) \cdot \xi} \tau^{|\beta|}(1-\tau)^{|\gamma|}(-1)^{|\gamma|} \times \\
\quad \times D_{\xi}^{\alpha}\left(\Psi_{N}-\Psi_{N+1}\right)(v, \xi) D_{\xi}^{\beta+\gamma-\alpha} \omega_{\beta \gamma}(x, y, \xi) d \xi .
\end{aligned}
$$


Thus,

$$
\begin{aligned}
K_{N}(x, y) \\
=\int e^{i(x-y) \cdot \xi}\left(\Psi_{N}-\Psi_{N+1}\right)(v, \xi)\left(a(x, y, \xi)-\sum_{j=0}^{N} p_{j}(v, \xi)\right) d \xi \\
=\int e^{i(x-y) \cdot \xi}\left(\Psi_{N}-\Psi_{N+1}\right)(v, \xi)\left(a(v, v, \xi)-p_{0}(v, \xi)\right) d \xi+ \\
+\sum_{j=1}^{N}\left(\sum_{|\beta+\gamma|=j} \sum_{\alpha \leq \beta+\gamma} \frac{(\beta+\gamma) !}{\beta ! \gamma !} \frac{1}{\alpha !(\beta+\gamma-\alpha) !} \times\right. \\
\quad \times \int e^{i(x-y) \cdot \xi} \tau^{|\beta|}(1-\tau)^{|\gamma|}(-1)^{|\gamma|} D_{\xi}^{\alpha}\left(\Psi_{N}-\Psi_{N+1}\right)(v, \xi) \times \\
\left.\quad \times\left(\partial_{x}^{\beta} \partial_{y}^{\gamma} D_{\xi}^{\beta+\gamma-\alpha} a\right)(v, v, \xi) d \xi-\int e^{i(x-y) \cdot \xi}\left(\Psi_{N}-\Psi_{N+1}\right)(v, \xi) p_{j}(v, \xi) d \xi\right)+ \\
+\quad \sum_{|\beta+\gamma|=N+1 \alpha \leq \beta+\gamma} \frac{1}{\beta ! \gamma !} \frac{1}{\alpha !(\beta+\gamma-\alpha) !} \int e^{i(x-y) \cdot \xi} \tau^{|\beta|}(1-\tau)^{|\gamma|}(-1)^{|\gamma|} \times \\
\quad \times D_{\xi}^{\alpha}\left(\Psi_{N}-\Psi_{N+1}\right)(v, \xi) D_{\xi}^{\beta+\gamma-\alpha} \omega_{\beta \gamma}(x, y, \xi) d \xi .
\end{aligned}
$$

Look at the three addends above. According to the change of variables we have made, we see that $p_{0}(v, \xi)=a(v, v, \xi)$, and hence, the first integral is equal to 0 . On the other hand, when $\alpha=0$, the first part of the second addend equals $\sum_{|\beta+\gamma|=j} \frac{(-1)^{|\gamma|}}{\beta ! \gamma !} \int e^{i(x-y) \cdot \xi} \tau^{|\beta|}(1-\tau)^{|\gamma|}\left(\Psi_{N}-\Psi_{N+1}\right)(v, \xi)\left(\partial_{x}^{\beta} \partial_{y}^{\gamma} D_{\xi}^{\beta+\gamma} a\right)(v, v, \xi) d \xi$, and by the definition of $p_{j}$, we see that the second part coincides with:

$$
\begin{aligned}
\int e^{i(x-y) \cdot \xi}\left(\Psi_{N}-\Psi_{N+1}\right)(v, \xi) p_{j}(v, \xi) d \xi \\
=\sum_{|\beta+\gamma|=j} \frac{1}{\beta ! \gamma !} \int e^{i(x-y) \cdot \xi} \tau^{|\beta|}(1-\tau)^{|\gamma|}(-1)^{|\gamma|} \times \\
\quad \times\left(\Psi_{N}-\Psi_{N+1}\right)(v, \xi)\left(\partial_{x}^{\beta} \partial_{y}^{\gamma} D_{\xi}^{\beta+\gamma} a\right)(v, v, \xi) d \xi
\end{aligned}
$$

Hence, we have obtained that $K_{N}(x, y)=\sum_{|\beta+\gamma|=1}^{N} A_{\beta \gamma}^{N}+Q_{N}$, being

$$
\begin{aligned}
A_{\beta \gamma}^{N}(x, y):= & \sum_{0 \neq \alpha \leq \beta+\gamma} \frac{(\beta+\gamma) !}{\beta ! \gamma !} \frac{1}{\alpha !(\beta+\gamma-\alpha) !} \int e^{i(x-y) \cdot \xi} \tau^{|\beta|}(1-\tau)^{|\gamma|}(-1)^{|\gamma|} \times \\
& \times D_{\xi}^{\alpha}\left(\Psi_{N}-\Psi_{N+1}\right)(v, \xi)\left(\partial_{x}^{\beta} \partial_{y}^{\gamma} D_{\xi}^{\beta+\gamma-\alpha} a\right)(v, v, \xi) d \xi,
\end{aligned}
$$


and

$$
\begin{aligned}
Q_{N}(x, y):= & \sum_{|\beta+\gamma|=N+1} \sum_{\alpha \leq \beta+\gamma} \frac{(\beta+\gamma) !}{\beta ! \gamma !} \frac{1}{\alpha !(\beta+\gamma-\alpha) !} \tau^{|\beta|}(1-\tau)^{|\gamma|}(-1)^{|\gamma|} \times \\
& \times \int e^{i(x-y) \cdot \xi} D_{\xi}^{\alpha}\left(\Psi_{N}-\Psi_{N+1}\right)(v, \xi) D_{\xi}^{\beta+\gamma-\alpha} \omega_{\beta \gamma}(x, y, \xi) d \xi .
\end{aligned}
$$

So $\sum_{r=1}^{N} K_{r}(x, y)=\sum_{r=1}^{N} \sum_{|\beta+\gamma|=1}^{r} A_{\beta \gamma}^{r}+\sum_{r=1}^{N} Q_{r}$. Since $\sum_{r=1}^{N} \sum_{|\beta+\gamma|=1}^{r}=$ $\sum_{|\beta+\gamma|=1}^{N} \sum_{r=|\beta+\gamma|}^{N}$, we obtain

$$
\begin{aligned}
\sum_{r=1}^{N} \sum_{|\beta+\gamma|=1}^{r} A_{\beta \gamma}^{r}(x, y) & \\
= & \sum_{|\beta+\gamma|=1}^{N} \sum_{r=|\beta+\gamma|}^{N} \sum_{0 \neq \alpha \leq \beta+\gamma} \frac{(\beta+\gamma) !}{\beta ! \gamma !} \frac{1}{\alpha !(\beta+\gamma-\alpha) !} \int e^{i(x-y) \cdot \xi} \tau^{|\beta|}(1-\tau)^{|\gamma|} \times \\
& \times(-1)^{|\gamma|} D_{\xi}^{\alpha}\left(\Psi_{r}-\Psi_{r+1}\right)(v, \xi)\left(\partial_{x}^{\beta} \partial_{y}^{\gamma} D_{\xi}^{\beta+\gamma-\alpha} a\right)(v, v, \xi) d \xi \\
= & \sum_{|\beta+\gamma|=1}^{N} \sum_{0 \neq \alpha \leq \beta+\gamma} \frac{(\beta+\gamma) !}{\beta ! \gamma !} \frac{1}{\alpha !(\beta+\gamma-\alpha) !} \int e^{i(x-y) \cdot \xi} \tau^{|\beta|}(1-\tau)^{|\gamma|}(-1)^{|\gamma|} \times \\
& \times D_{\xi}^{\alpha}\left(\Psi_{|\beta+\gamma|}-\Psi_{N+1}\right)(v, \xi)\left(\partial_{x}^{\beta} \partial_{y}^{\gamma} D_{\xi}^{\beta+\gamma-\alpha} a\right)(v, v, \xi) d \xi .
\end{aligned}
$$

Therefore, $\sum_{r=1}^{N} \sum_{|\beta+\gamma|=1}^{r} A_{\beta \gamma}^{r}=\sum_{j=1}^{N} I_{j}-W_{N}$, with

$$
\begin{aligned}
I_{j}(x, y):= & \sum_{|\beta+\gamma|=j} \sum_{0 \neq \alpha \leq \beta+\gamma} \frac{(\beta+\gamma) !}{\beta ! \gamma !} \frac{1}{\alpha !(\beta+\gamma-\alpha) !} \int e^{i(x-y) \cdot \xi} \tau^{|\beta|}(1-\tau)^{|\gamma|} \times \\
& \times(-1)^{|\gamma|} D_{\xi}^{\alpha} \Psi_{j}(v, \xi)\left(\partial_{x}^{\beta} \partial_{y}^{\gamma} D_{\xi}^{\beta+\gamma-\alpha} a\right)(v, v, \xi) d \xi
\end{aligned}
$$

and

$$
\begin{aligned}
W_{N}(x, y):= & \sum_{|\beta+\gamma|=1}^{N} \sum_{0 \neq \alpha \leq \beta+\gamma} \frac{(\beta+\gamma) !}{\beta ! \gamma !} \frac{1}{\alpha !(\beta+\gamma-\alpha) !} \int e^{i(x-y) \cdot \xi} \tau^{|\beta|}(1-\tau)^{|\gamma|} \times \\
& \times(-1)^{|\gamma|} D_{\xi}^{\alpha} \Psi_{N+1}(v, \xi)\left(\partial_{x}^{\beta} \partial_{y}^{\gamma} D_{\xi}^{\beta+\gamma-\alpha} a\right)(v, v, \xi) d \xi
\end{aligned}
$$

Thus, we can write the partial sums of the kernel by

$$
\sum_{j=0}^{N} K_{j}=K_{0}+\sum_{j=1}^{N} I_{j}+\sum_{j=1}^{N} Q_{j}-W_{N},
$$


where

$$
\begin{aligned}
I_{j}(x, y):= & \sum_{|\beta+\gamma|=j} \sum_{0 \neq \alpha \leq \beta+\gamma} \frac{(\beta+\gamma) !}{\beta ! \gamma !} \frac{1}{\alpha !(\beta+\gamma-\alpha) !} \int e^{i(x-y) \cdot \xi} \tau^{|\beta|} \times \\
& \times(1-\tau)^{|\gamma|}(-1)^{|\gamma|} D_{\xi}^{\alpha} \Psi_{j}(v, \xi)\left(\partial_{x}^{\beta} \partial_{y}^{\gamma} D_{\xi}^{\beta+\gamma-\alpha} a\right)(v, v, \xi) d \xi \\
Q_{j}(x, y):= & \sum_{|\beta+\gamma|=j+1} \sum_{\alpha \leq \beta+\gamma} \frac{(\beta+\gamma) !}{\beta ! \gamma !} \frac{1}{\alpha !(\beta+\gamma-\alpha) !} \tau^{|\beta|}(1-\tau)^{|\gamma|}(-1)^{|\gamma|} \times \\
& \times \int e^{i(x-y) \cdot \xi} D_{\xi}^{\alpha}\left(\Psi_{j}-\Psi_{j+1}\right)(v, \xi) D_{\xi}^{\beta+\gamma-\alpha} \omega_{\beta \gamma}(x, y, \xi) d \xi \\
\omega_{\beta \gamma}(x, y, \xi):= & (j+1) \int_{0}^{1}\left(\partial_{x}^{\beta} \partial_{y}^{\gamma} a\right)(v+t \tau w, v-(1-\tau) t w, \xi)(1-t)^{j} d t \\
W_{N}(x, y):= & \sum_{|\beta+\gamma|=1}^{N} \sum_{0 \neq \alpha \leq \beta+\gamma} \frac{(\beta+\gamma) !}{\beta ! \gamma !} \frac{1}{\alpha !(\beta+\gamma-\alpha) !} \int e^{i(x-y) \cdot \xi} \tau^{|\beta|} \times \\
& \times(1-\tau)^{|\gamma|}(-1)^{|\gamma|} D_{\xi}^{\alpha} \Psi_{N+1}(v, \xi)\left(\partial_{x}^{\beta} \partial_{y}^{\gamma} D_{\xi}^{\beta+\gamma-\alpha} a\right)(v, v, \xi) d \xi .
\end{aligned}
$$

It is easy to see that $K_{0} \in \mathcal{S}_{\omega}\left(\mathbb{R}^{2 d}\right)$. Indeed, we have

$$
K_{0}(x, y)=\int e^{i(x-y) \cdot \xi}\left(1-\Psi_{1}\right)((1-\tau) x+\tau y, \xi)(a(x, y, \xi)-a(x, x, \xi)) d \xi
$$

Since $1-\Psi_{1} \in \mathcal{S}_{\omega}\left(\mathbb{R}^{2 d}\right)$, following the proof of Lemma 1.11 (with an integration by parts with formula (1.7)), for all $\lambda>0$ there exists $C_{\lambda}>0$ such that $(k$ is as in $(2.16)$ )

$$
\left|D_{x}^{\alpha} D_{\xi}^{\beta} K_{0}(x, y)\right| \leq C_{\lambda} e^{\lambda \varphi^{*}\left(\frac{|\alpha+\beta|}{\lambda}\right)} e^{-\lambda L^{2} \omega((1-\tau) x+\tau y)} e^{-\lambda L^{k+2} \omega(y-x)},
$$

for all $\alpha, \beta \in \mathbb{N}_{0}^{d}, x, y \in \mathbb{R}^{d}$. An application of Lemma 2.22 gives the result. Therefore, by Lemma $2.19,(1-\chi) K_{0} \in \mathcal{S}_{\omega}\left(\mathbb{R}^{2 d}\right)$. In what follows, we only treat the case $m \geq 0$ (the other case is easier and follows in the same way).

First step. To show that $\sum_{j=1}^{\infty} I_{j}(x, y) \in \mathcal{S}_{\omega}\left(\mathbb{R}^{2 d}\right)$, we compute $D_{x}^{\theta} D_{y}^{\epsilon} I_{j}(x, y)$ for $\theta, \epsilon \in \mathbb{N}_{0}^{d}$ :

$$
\begin{aligned}
& D_{x}^{\theta} D_{y}^{\epsilon} I_{j}(x, y) \\
& =\sum_{|\beta+\gamma|=j} \sum_{0 \neq \alpha \leq \beta+\gamma} \frac{(\beta+\gamma) !}{\beta ! \gamma !} \frac{1}{\alpha !(\beta+\gamma-\alpha) !} \sum_{\substack{\theta_{1}+\theta_{2}+\theta_{3}=\theta \\
\epsilon_{1}+\epsilon_{2}+\epsilon_{3}=\epsilon}} \frac{\theta !}{\theta_{1} ! \theta_{2} ! \theta_{3} !} \frac{\epsilon !}{\epsilon_{1} ! \epsilon_{2} ! \epsilon_{3} !} \times \\
& \times(-1)^{\left|\gamma+\epsilon_{1}\right|} \tau^{\left|\beta+\epsilon_{2}\right|}(1-\tau)^{\left|\gamma+\theta_{2}\right|} \int e^{i(x-y) \cdot \xi} \xi^{\theta_{1}+\epsilon_{1}} \times \\
& \times D_{x}^{\theta_{2}} D_{y}^{\epsilon_{2}} D_{\xi}^{\alpha} \Psi_{j}(v, \xi) D_{x}^{\theta_{3}} D_{y}^{\epsilon_{3}}\left(\partial_{x}^{\beta} \partial_{y}^{\gamma} D_{\xi}^{\beta+\gamma-\alpha} a\right)(v, v, \xi) d \xi .
\end{aligned}
$$


An integration by parts with formula (1.7) (for some power $s \in \mathbb{N}$, determined later) yields

$$
\begin{aligned}
e^{i(x-y) \cdot \xi} \frac{1}{G^{s}(y-x)} G^{s}\left(D_{\xi}\right)\left\{\xi^{\theta_{1}+\epsilon_{1}} D_{x}^{\theta_{2}} D_{y}^{\epsilon_{2}} D_{\xi}^{\alpha} \Psi_{j}(v, \xi) \times\right. \\
\left.\times D_{x}^{\theta_{3}} D_{y}^{\epsilon_{3}}\left(\partial_{x}^{\beta} \partial_{y}^{\gamma} D_{\xi}^{\beta+\gamma-\alpha} a\right)(v, v, \xi)\right\} \\
=e^{i(x-y) \cdot \xi} \frac{1}{G^{s}(y-x)} \sum_{\eta \in \mathbb{N}_{0}^{d}} b_{\eta} \sum_{\eta_{1}+\eta_{2}+\eta_{3}=\eta} \frac{\eta !}{\eta_{1} ! \eta_{2} ! \eta_{3} !} \frac{\left(\theta_{1}+\epsilon_{1}\right) !}{\left(\theta_{1}+\epsilon_{1}-\eta_{1}\right) !} \xi^{\theta_{1}+\epsilon_{1}-\eta_{1}} \times \\
\quad \times D_{x}^{\theta_{2}} D_{y}^{\epsilon_{2}} D_{\xi}^{\alpha+\eta_{2}} \Psi_{j}(v, \xi) D_{x}^{\theta_{3}} D_{y}^{\epsilon_{3}}\left(\partial_{x}^{\beta} \partial_{y}^{\gamma} D_{\xi}^{\beta+\gamma-\alpha+\eta_{3}} a\right)(v, v, \xi) .
\end{aligned}
$$

Therefore,

$$
\begin{aligned}
D_{x}^{\theta} D_{y}^{\epsilon} I_{j}(x, y)= & \sum_{|\beta+\gamma|=j} \sum_{0 \neq \alpha \leq \beta+\gamma} \frac{(\beta+\gamma) !}{\beta ! \gamma !} \frac{1}{\alpha !(\beta+\gamma-\alpha) !} \frac{1}{G^{s}(y-x)} \sum_{\eta \in \mathbb{N}_{0}^{d}} b_{\eta} \times \\
& \times \sum_{\begin{array}{c}
\theta_{1}+\theta_{2}+\theta_{3}=\theta \\
\epsilon_{1}+\epsilon_{2}+\epsilon_{3}=\epsilon \\
\eta_{1}+\eta_{2}+\eta_{3}=\eta
\end{array}}(-1)^{\left|\gamma+\epsilon_{1}\right|} \frac{\theta !}{\theta_{1} ! \theta_{2} ! \theta_{3} !} \frac{\epsilon !}{\epsilon_{1} ! \epsilon_{2} ! \epsilon_{3} !} \frac{\eta !}{\eta_{1} ! \eta_{2} ! \eta_{3} !} \times \\
& \times \frac{\left(\theta_{1}+\epsilon_{1}\right) !}{\left(\theta_{1}+\epsilon_{1}-\eta_{1}\right) !} \tau^{\left|\beta+\epsilon_{2}\right|}(1-\tau)^{\left|\gamma+\theta_{2}\right|} \int e^{i(x-y) \cdot \xi} \xi^{\theta_{1}+\epsilon_{1}-\eta_{1}} \times \\
& \times D_{x}^{\theta_{2}} D_{y}^{\epsilon_{2}} D_{\xi}^{\alpha+\eta_{2}} \Psi_{j}(v, \xi) D_{x}^{\theta_{3}} D_{y}^{\epsilon_{3}}\left(\partial_{x}^{\beta} \partial_{y}^{\gamma} D_{\xi}^{\beta+\gamma-\alpha+\eta_{3}} a\right)(v, v, \xi) d \xi
\end{aligned}
$$

Fix $\lambda>0$ and take $n \geq \lambda$ to be determined later. Fix $\tilde{p} \in \mathbb{N}$ such that $\max \{2,2|1-\tau|, 2|\tau|\} \leq e^{\rho \widetilde{p}}$ and $q \in \mathbb{N}$ such that $2^{q} \geq 3 R$. Write $\tilde{n} \geq n$ satisfying

$$
\widetilde{n} \geq \max \left\{n L^{\widetilde{p}}, \lambda L^{\widetilde{p}+2}, n L^{3}, \frac{L^{q}}{\rho}\left(\lambda L^{\widetilde{p}+2}+m L+1\right)+1\right\} .
$$

By Definition 1.3 and Corollary 2.9, for that $\widetilde{n}$ there exist $C_{\widetilde{n}}, D_{\widetilde{n}}>0$ such that for each $v, \xi \in \mathbb{R}^{d}$ we have by the chain rule

$$
\begin{aligned}
& \left|D_{x}^{\theta_{3}} D_{y}^{\epsilon_{3}}\left(\partial_{x}^{\beta} \partial_{y}^{\gamma} D_{\xi}^{\beta+\gamma-\alpha+\eta_{3}} a\right)(v, v, \xi)\right| \\
& \leq C_{\widetilde{n}}\langle(v, \xi)\rangle^{-\rho\left|2 \beta+2 \gamma-\alpha+\theta_{3}+\epsilon_{3}+\eta_{3}\right|}(2 \max \{|1-\tau|,|\tau|\})^{\left|\theta_{3}+\epsilon_{3}\right|} \times \\
& \quad \times e^{8 \widetilde{n} \rho \varphi^{*}\left(\frac{\left|2 \beta+2 \gamma-\alpha+\theta_{3}+\epsilon_{3}+\eta_{3}\right|}{8 \widetilde{n}}\right)} e^{m \omega(v, v, \xi)} ; \\
& \left|D_{x}^{\theta_{2}} D_{y}^{\epsilon_{2}} D_{\xi}^{\alpha+\eta_{2}} \Psi_{j}(v, \xi)\right| \leq D_{\widetilde{n}}\langle(v, \xi)\rangle^{-\rho\left|\alpha+\theta_{2}+\epsilon_{2}+\eta_{2}\right|} e^{8 \widetilde{n} \rho \varphi^{*}\left(\frac{\left|\alpha+\theta_{2}+\epsilon_{2}+\eta_{2}\right|}{8 \tilde{n}}\right)} .
\end{aligned}
$$

Since $|\beta+\gamma|=j$, we obtain

$$
\langle(v, \xi)\rangle^{-\rho\left|2 \beta+2 \gamma-\alpha+\theta_{3}+\epsilon_{3}+\eta_{3}\right|}\langle(v, \xi)\rangle^{-\rho\left|\alpha+\theta_{2}+\epsilon_{2}+\eta_{2}\right|} \leq\left(\langle(v, \xi)\rangle^{-j}\right)^{2 \rho},
$$


and as in $(0.4), m \omega(v, v, \xi) \leq m \omega(\sqrt{2} \max \{|v|,|\xi|\}) \leq m L \omega(v)+m L \omega(\xi)+m L$. By $(0.7)$ we have (if $|\xi| \geq 1$ )

$$
|\xi|^{\left|\theta_{1}+\epsilon_{1}-\eta_{1}\right|} \leq e^{\lambda L^{\widetilde{p}+2} \varphi^{*}\left(\frac{\left|\theta_{1}+\epsilon_{1}-\eta_{1}\right|}{\lambda L^{\tilde{p}+2}}\right)} e^{\lambda L^{\widetilde{p}+2} \omega(\xi)} .
$$

By the choice of $\widetilde{p} \in \mathbb{N}$ and from (0.12), there exists $E_{\lambda}>0$ such that

$$
\begin{aligned}
& (2 \max \{|1-\tau|,|\tau|\})^{\left|\theta_{3}+\epsilon_{3}\right|}|\tau|^{\left|\beta+\epsilon_{2}\right|}|1-\tau|^{\left|\gamma+\theta_{2}\right|} \frac{(\beta+\gamma) !}{\beta ! \gamma !} \frac{\left(\theta_{1}+\epsilon_{1}\right) !}{\left(\theta_{1}+\epsilon_{1}-\eta_{1}\right) !} \\
& \leq e^{\rho \widetilde{p}|2 \beta+2 \gamma|} e^{\rho \widetilde{p}|\theta+\epsilon|} E_{\lambda} e^{\lambda L^{\widetilde{p}+2} \varphi^{*}\left(\frac{\left|\eta_{1}\right|}{\lambda L^{\tilde{p}+2}}\right)} .
\end{aligned}
$$

By Lemma 0.8 and the selection of $\widetilde{n}$ (recall that $|\beta+\gamma|=j$ ), we deduce

$$
\begin{aligned}
& e^{\lambda L^{\widetilde{p}+2} \varphi^{*}\left(\frac{\left|\eta_{1}\right|}{\lambda L^{\tilde{p}+2}}\right)} e^{\lambda L^{\widetilde{p}+2} \varphi^{*}\left(\frac{\left|\theta_{1}+\epsilon_{1}-\eta_{1}\right|}{\lambda L^{\widetilde{p}+2}}\right)} e^{8 \widetilde{n} \rho \varphi^{*}\left(\frac{\left|\alpha+\theta_{2}+\epsilon_{2}+\eta_{2}\right|}{8 \tilde{n}}\right)} e^{8 \widetilde{n} \rho \varphi^{*}\left(\frac{\left|2 \beta+2 \gamma-\alpha+\theta_{3}+\epsilon_{3}+\eta_{3}\right|}{8 \tilde{n}}\right)} \\
& \leq e^{\lambda L^{\widetilde{p}+2} \varphi^{*}\left(\frac{\left|\theta_{1}+\epsilon_{1}\right|}{\lambda L \tilde{p}+2}\right)} e^{8 \widetilde{n} \rho \varphi^{*}\left(\frac{\left|2 \beta+2 \gamma+\theta_{2}+\theta_{3}+\epsilon_{2}+\epsilon_{3}+\eta_{2}+\eta_{3}\right|}{8 \tilde{n}}\right)} \\
& \leq e^{\lambda L^{\widetilde{p}+2} \varphi^{*}\left(\frac{\left|\theta_{1}+\epsilon_{1}\right|}{\lambda L^{\tilde{p}+2}}\right)} e^{4 \widetilde{n} \rho \varphi^{*}\left(\frac{|2 \beta+2 \gamma|}{4 \tilde{n}}\right)} e^{4 \widetilde{n} \rho \varphi^{*}\left(\frac{\left|\theta_{2}+\theta_{3}+\epsilon_{2}+\epsilon_{3}+\eta_{2}+\eta_{3}\right|}{4 \tilde{n}}\right)} \\
& \leq e^{\lambda L^{\widetilde{p}+2} \varphi^{*}\left(\frac{\left|\theta_{1}+\epsilon_{1}\right|}{\lambda L^{\tilde{p}+2}}\right)}\left(e^{2 \widetilde{n} \varphi^{*}\left(\frac{j}{2 \widetilde{n}}\right)}\right)^{2 \rho} e^{\lambda L^{\widetilde{p}+2} \varphi^{*}\left(\frac{\left|\theta_{2}+\theta_{3}+\epsilon_{2}+\epsilon_{3}\right|}{\lambda L^{\tilde{p}}+2}\right)} e^{\widetilde{n} \varphi^{*}\left(\frac{\left|\eta_{2}+\eta_{3}\right|}{\widetilde{n}}\right)} \\
& \leq e^{\lambda L^{\widetilde{p}+2} \varphi^{*}\left(\frac{|\theta+\epsilon|}{\lambda L^{\widetilde{p}+2}}\right)}\left(e^{2 \widetilde{n} \varphi^{*}\left(\frac{j}{2 \widetilde{n}}\right)}\right)^{2 \rho} e^{n L^{3} \varphi^{*}\left(\frac{|\eta|}{n L^{3}}\right)} .
\end{aligned}
$$

Moreover, since $\sum \begin{gathered}\theta_{1}+\theta_{2}+\theta_{3}=\theta \\ \epsilon_{1}+\epsilon_{2}+\epsilon_{3}=\epsilon\end{gathered} \frac{\theta !}{\theta_{1} ! \theta_{2} ! \theta_{3} !} \frac{\epsilon !}{\epsilon_{1} ! \epsilon_{2} ! \epsilon_{3} !} \leq e^{2|\theta+\epsilon|}$, we obtain by $(0.10)$,

$$
e^{\lambda L^{\widetilde{p}+2} \varphi^{*}\left(\frac{|\theta+\epsilon|}{\lambda L^{\tilde{p}+2}}\right)} e^{\rho \widetilde{p}|\theta+\epsilon|} \sum_{\substack{\theta_{1}+\theta_{2}+\theta_{3}=\theta \\ \epsilon_{1}+\epsilon_{2}+\epsilon_{3}=\epsilon}} \frac{\theta !}{\theta_{1} ! \theta_{2} ! \theta_{3} !} \frac{\epsilon !}{\epsilon_{1} ! \epsilon_{2} ! \epsilon_{3} !} \leq e^{\lambda \varphi^{*}\left(\frac{|\theta+\epsilon|}{\lambda}\right)} e^{\lambda \sum_{p=1}^{\tilde{p}+2} L^{p}}
$$

From Corollaries 0.23 and 0.20 there are $C_{1}, C_{2}, C_{3}>0$ such that

$$
\left|b_{\eta}\right| \leq e^{s C_{1}} e^{-s C_{1} \varphi^{*}\left(\frac{|\eta|}{s C_{1}}\right)}, \quad\left|\frac{1}{G^{s}(y-x)}\right| \leq C_{3}^{s} e^{-s C_{2} \omega(y-x)} .
$$

On the other hand, we recall that if the derivatives of $\Psi_{j}(v, \xi)$ do not vanish, then we can assume $2 A_{\widetilde{n}, j} \leq|(v, \xi)| \leq 3 A_{\widetilde{n}, j}$ (see (2.4)). So, in particular,

$$
\frac{1}{e} e^{\frac{\widetilde{n}}{j} \varphi^{*}\left(\frac{j}{\tilde{n}}\right)} \leq \frac{2}{3} e^{\frac{\widetilde{n}}{j} \varphi^{*}\left(\frac{j}{\tilde{n}}\right)} \leq \frac{|(v, \xi)|}{3 R} \leq e^{\frac{\widetilde{n}}{j} \varphi^{*}\left(\frac{j}{\tilde{n}}\right)} .
$$

By Lemma 2.21, we then have, for $j \in \mathbb{N}$ large enough,

$$
\left(e^{2 \widetilde{n} \varphi^{*}\left(\frac{j}{2 n}\right)}\right)^{2 \rho} \leq\left(e^{-(\widetilde{n}-1) \omega\left(\frac{(v, \xi)}{3 R}\right)}\right)^{2 \rho}\left(e^{\widetilde{n} \varphi^{*}\left(\frac{j}{n}\right)}\right)^{2 \rho} .
$$


Now, using $q$ times property $(\alpha)$ of Definition 0.3 (where $q$ is such that $2^{q} \geq$ $3 R$ ), we have

$$
\omega\left(\frac{1}{3 R}(v, \xi)\right) \geq \frac{1}{L^{q}} \omega\left(\frac{2^{q}}{3 R}(v, \xi)\right)-\frac{1}{L^{q-1}}-\frac{1}{L^{q-2}}-\cdots-1 \geq \frac{1}{L^{q}} \omega(v, \xi)-q .
$$

Therefore, since $2 \omega(v, \xi) \geq \omega(v)+\omega(\xi)$, we obtain

$$
\begin{aligned}
\left(e^{-(\widetilde{n}-1) \omega\left(\frac{(v, \xi)}{3 R}\right)}\right)^{2 \rho} & \leq\left(e^{-\frac{\widetilde{n}-1}{L^{q}} \omega(v, \xi)}\right)^{2 \rho} e^{q(\widetilde{n}-1) 2 \rho} \\
& \leq\left(e^{-\frac{\widetilde{n}-1}{2 L^{q}} \omega(v)}\right)^{2 \rho}\left(e^{-\frac{\widetilde{n}-1}{2 L^{q}} \omega(\xi)}\right)^{2 \rho} e^{q(\widetilde{n}-1) 2 \rho},
\end{aligned}
$$

and we get (since $\sum_{\eta_{1}+\eta_{2}+\eta_{3}=\eta} \frac{\eta !}{\eta_{1} ! \eta_{2} ! \eta_{3} !}=3^{|\eta|}$ ) that $\left|D_{x}^{\theta} D_{y}^{\epsilon} I_{j}(x, y)\right|$ is less than or equal to

$$
\begin{aligned}
& \sum_{|\beta+\gamma|=j} \sum_{0 \neq \alpha \leq \beta+\gamma} e^{\rho \widetilde{p}|2 \beta+2 \gamma|} \frac{1}{\alpha !(\beta+\gamma-\alpha) !} e^{\lambda \varphi^{*}\left(\frac{|\theta+\epsilon|}{\lambda}\right)} e^{\lambda \sum_{p=1}^{\tilde{p}+2} L^{p}} e^{s C_{1}} \times \\
& \times\left(\sum_{\eta \in \mathbb{N}_{0}^{d}} e^{-s C_{1} \varphi^{*}\left(\frac{|\eta|}{s C_{1}}\right)} e^{n L^{3} \varphi^{*}\left(\frac{|\eta|}{n L^{3}}\right)} 3^{|\eta|}\right) E_{\lambda} C_{3}^{s} e^{-s C_{2} \omega(y-x)} \times \\
& \quad \times \int e^{\lambda L^{\tilde{p}+2} \omega(\xi)} D_{n}\left(\langle(v, \xi)\rangle^{-j}\right)^{2 \rho} C_{n} e^{m L \omega(v)} e^{m L \omega(\xi)} e^{m L} \times \\
& \quad \times\left(e^{\widetilde{n} \varphi^{*}\left(\frac{j}{n}\right)}\right)^{2 \rho}\left(e^{-\frac{\widetilde{n}-1}{2 L^{q}} \omega(v)}\right)^{2 \rho}\left(e^{-\frac{\widetilde{n}-1}{2 L^{q}} \omega(\xi)}\right)^{2 \rho} e^{q(\widetilde{n}-1) 2 \rho} d \xi .
\end{aligned}
$$

By the choice of $\widetilde{n}$, since $\widetilde{n} \geq \frac{L^{q}}{\rho}\left(\lambda L^{\widetilde{p}+2}+m L+1\right)+1$, we have

$$
e^{-\frac{\widetilde{n}-1}{L q} \rho \omega(\xi)} e^{\lambda L^{\widetilde{p}+2} \omega(\xi)} e^{m L \omega(\xi)} \leq e^{-\omega(\xi)}
$$

which ensures the convergence of the integral in $\xi$ which defines $D_{x}^{\theta} D_{y}^{\epsilon} I_{j}(x, y)$. Furthermore, since $\widetilde{n} \geq \frac{L^{q}}{\rho}\left(\lambda L^{2}+m L\right)+1$, we see that

$$
e^{-\frac{\widetilde{n}-1}{L^{q}} \rho \omega(v)} e^{m L \omega(v)} \leq e^{-\lambda L^{2} \omega(v)}
$$

Then, according to Lemma 2.22, it is enough to take $s \in \mathbb{N}$ such that

$$
s C_{2} \geq \lambda L^{k+2}
$$

where $k \in \mathbb{N}_{0}$ is as in (2.16), in order to obtain

$$
e^{-\lambda L^{2} \omega(v)} e^{-s C_{2} \omega(y-x)} \leq e^{-\lambda \omega(x, y)} e^{\lambda \sum_{t=1}^{k+2} L^{t}} .
$$

For the convergence of the series depending on $\eta \in \mathbb{N}_{0}^{d}$, it is enough to assume that $n \geq s C_{1}$ (see (1.14)). Since $\widetilde{n} \geq n L^{\widetilde{p}}$, using formula (0.10) we obtain 
(since $|\beta+\gamma|=j)$

$$
\begin{aligned}
\left(\langle(v, \xi)\rangle^{-j}\right)^{2 \rho}\left(e^{\widetilde{p} j}\right)^{2 \rho}\left(e^{n L^{\widetilde{p}} \varphi^{*}\left(\frac{j}{n L^{\widetilde{p}}}\right)}\right)^{2 \rho} & \leq\left(\langle(v, \xi)\rangle^{-j}\right)^{2 \rho}\left(e^{n \varphi^{*}\left(\frac{j}{n}\right)}\right)^{2 \rho} e^{2 n \rho \sum_{t=1}^{\widetilde{r}} L^{t}} \\
& \leq\left((2 R)^{-j}\right)^{2 \rho} e^{2 n \rho \sum_{t=1}^{\widetilde{p}} L^{t}} .
\end{aligned}
$$

Finally, the convergence of the series (by Lemma 0.1(8))

$$
\begin{aligned}
& \sum_{j=1}^{\infty} \sum_{|\beta+\gamma|=j} \sum_{0 \neq \alpha \leq \beta+\gamma} \frac{1}{\alpha !(\beta+\gamma-\alpha) !}\left((2 R)^{-j}\right)^{2 \rho} \leq \sum_{j=1}^{\infty} \frac{2^{j}}{(2 R)^{2 j \rho}} \sum_{|\beta+\gamma|=j} \frac{1}{(\beta+\gamma) !} \\
& \quad \leq \sum_{j=1}^{\infty} \frac{(2 d)^{j}}{(2 R)^{2 j \rho}} \sum_{|\beta+\gamma|=j} \frac{1}{|\beta+\gamma| !} \leq \sum_{j=1}^{\infty} \frac{1}{j !} \frac{\left(2 d^{2}\right)^{j}}{(2 R)^{2 \rho j}}
\end{aligned}
$$

shows that $\sum_{j=1}^{\infty} I_{j} \in \mathcal{S}_{\omega}\left(\mathbb{R}^{2 d}\right)$ and by Lemma $2.19,(1-\chi) \sum_{j=1}^{\infty} I_{j} \in \mathcal{S}_{\omega}\left(\mathbb{R}^{2 d}\right)$.

Second step. Since $\operatorname{supp}(1-\chi) \subseteq \Delta_{2 r}$, it is enough to estimate $\left|D_{x}^{\theta} D_{y}^{\epsilon} Q_{j}(x, y)\right|$ for $\theta, \epsilon \in \mathbb{N}_{0}^{d},(x, y) \in \Delta_{2 r}$. We have

$$
\begin{aligned}
D_{x}^{\theta} D_{y}^{\epsilon} Q_{j}(x, y) & =\sum_{|\beta+\gamma|=j+1} \sum_{\alpha \leq \beta+\gamma} \frac{(\beta+\gamma) !}{\beta ! \gamma !} \frac{1}{\alpha !(\beta+\gamma-\alpha) !} \sum_{\substack{\theta_{1}+\theta_{2}+\theta_{3}=\theta \\
\epsilon_{1}+\epsilon_{2}+\epsilon_{3}=\epsilon}} \frac{\theta !}{\theta_{1} ! \theta_{2} ! \theta_{3} !} \frac{\epsilon !}{\epsilon_{1} ! \epsilon_{2} ! \epsilon_{3} !} \times \\
& \times(-1)^{\left|\gamma+\epsilon_{1}\right|} \tau^{\left|\beta+\epsilon_{2}\right|}(1-\tau)^{\left|\gamma+\theta_{2}\right|} \int e^{i(x-y) \cdot \xi} \xi^{\theta_{1}+\epsilon_{1}} \times \\
& \times D_{x}^{\theta_{2}} D_{y}^{\epsilon_{2}} D_{\xi}^{\alpha}\left(\Psi_{j}-\Psi_{j+1}\right)(v, \xi) D_{x}^{\theta_{3}} D_{y}^{\epsilon_{3}}\left(D_{\xi}^{\beta+\gamma-\alpha} \omega_{\beta \gamma}\right)(x, y, \xi) d \xi
\end{aligned}
$$

where $\omega_{\beta \gamma}(x, y, \xi)$ is defined in (2.23). As in the first step, we use integration by parts given by formula (1.7) for a suitable power of $G(D), G^{s}(D)$, determined later. The integrand above then equals

$$
\begin{aligned}
e^{i(x-y)} \cdot \xi & \frac{1}{G^{s}(y-x)} G^{s}\left(D_{\xi}\right)\left\{\xi^{\theta_{1}+\epsilon_{1}} D_{x}^{\theta_{2}} D_{y}^{\epsilon_{2}} D_{\xi}^{\alpha}\left(\Psi_{j}-\Psi_{j+1}\right)(v, \xi) \times\right. \\
\times & \left.D_{x}^{\theta_{3}} D_{y}^{\epsilon_{3}}\left(D_{\xi}^{\beta+\gamma-\alpha} \omega_{\beta \gamma}\right)(x, y, \xi)\right\} \\
= & e^{i(x-y) \cdot \xi} \frac{1}{G^{s}(y-x)} \sum_{\eta \in \mathbb{N}_{0}^{d}} b_{\eta} \sum_{\eta_{1}+\eta_{2}+\eta_{3}=\eta} \frac{\eta !}{\eta_{1} ! \eta_{2} ! \eta_{3} !} \frac{\left(\theta_{1}+\epsilon_{1}\right) !}{\left(\theta_{1}+\epsilon_{1}-\eta_{1}\right) !} \xi^{\theta_{1}+\epsilon_{1}-\eta_{1}} \times \\
& \times D_{x}^{\theta_{2}} D_{y}^{\epsilon_{2}} D_{\xi}^{\alpha+\eta_{2}}\left(\Psi_{j}-\Psi_{j+1}\right)(v, \xi) D_{x}^{\theta_{3}} D_{y}^{\epsilon_{3}}\left(D_{\xi}^{\beta+\gamma-\alpha+\eta_{3}} \omega_{\beta \gamma}\right)(x, y, \xi) .
\end{aligned}
$$


Thus, we obtain

$$
\begin{aligned}
& D_{x}^{\theta} D_{y}^{\epsilon} Q_{j}(x, y) \\
&=\sum_{|\beta+\gamma|=j+1} \sum_{\alpha \leq \beta+\gamma} \frac{(\beta+\gamma) !}{\beta ! \gamma !} \frac{1}{\alpha !(\beta+\gamma-\alpha) !} \frac{1}{G^{s}(y-x)} \sum_{\eta \in \mathbb{N}_{0}^{d}} b_{\eta} \times \\
& \times \sum_{\substack{\theta_{1}+\theta_{2}+\theta_{3}=\theta \\
\epsilon_{1}+\epsilon_{2}+\epsilon_{3}=\epsilon \\
\eta_{1}+\eta_{2}+\eta_{3}=\eta}} \frac{\theta !}{\theta_{1} ! \theta_{2} ! \theta_{3} !} \frac{\epsilon !}{\epsilon_{1} ! \epsilon_{2} ! \epsilon_{3} !} \frac{\eta !}{\eta_{1} ! \eta_{2} ! \eta_{3} !} \frac{\left(\theta_{1}+\epsilon_{1}\right) !}{\left(\theta_{1}+\epsilon_{1}-\eta_{1}\right) !}(-1)^{\left|\gamma+\epsilon_{1}\right|} \tau^{\left|\beta+\epsilon_{2}\right|} \times \\
& \times(1-\tau)^{\left|\gamma+\theta_{2}\right|} \int e^{i(x-y) \cdot \xi} \xi^{\theta_{1}+\epsilon_{1}-\eta_{1}} D_{x}^{\theta_{2}} D_{y}^{\epsilon_{2}} D_{\xi}^{\alpha+\eta_{2}}\left(\Psi_{j}-\Psi_{j+1}\right)(v, \xi) \times \\
& \times D_{x}^{\theta_{3}} D_{y}^{\epsilon_{3}}\left(D_{\xi}^{\beta+\gamma-\alpha+\eta_{3}} \omega_{\beta \gamma}\right)(x, y, \xi) d \xi .
\end{aligned}
$$

We estimate the derivatives of $\omega_{\beta \gamma}$. Since $v+t \tau w=(1-\tau+t \tau) x+\tau(1-t) y$ and $v-(1-\tau) t w=(1-\tau)(1-t) x+(\tau+t-\tau t) y$, we have, by $(2.23)$,

$$
\begin{aligned}
D_{x}^{\theta_{3}} D_{y}^{\epsilon_{3}} & \left(D_{\xi}^{\beta+\gamma-\alpha+\eta_{3}} \omega_{\beta \gamma}\right)(x, y, \xi) \\
= & (j+1) \int_{0}^{1}(1-t)^{j} \sum_{\substack{\widetilde{\theta_{3}} \leq \theta_{3} \\
\tilde{\epsilon_{3}} \leq \epsilon_{3}}}\left(\begin{array}{c}
\theta_{3} \\
\widetilde{\theta_{3}}
\end{array}\right)\left(\begin{array}{c}
\epsilon_{3} \\
\widetilde{\epsilon_{3}}
\end{array}\right) \times \\
& \times|1-\tau+t \tau|^{\left|\widetilde{\theta_{3}}\right|}(|1-\tau||1-t|)^{\left|\theta_{3}-\widetilde{\theta_{3}}\right|}(|\tau||1-t|)^{\left|\widetilde{\epsilon_{3}}\right|}|\tau+t-t \tau|^{\mid \epsilon_{3}-\widetilde{\epsilon_{3} \mid} \times} \\
& \times D_{x}^{\widetilde{\theta_{3}}} D_{y}^{\widetilde{\epsilon_{3}}} D_{x}^{\theta_{3}-\widetilde{\theta_{3}}} D_{y}^{\epsilon_{3}-\widetilde{\epsilon_{3}}} \partial_{x}^{\beta} \partial_{y}^{\gamma} D_{\xi}^{\beta+\gamma-\alpha+\eta_{3}} a(v+t \tau w, v-(1-\tau) t w, \xi) d t .
\end{aligned}
$$

We take in this step $\widetilde{p} \in \mathbb{N}_{0}$ such that $\rho \widetilde{p} \geq 1$ and

$$
\max \left\{2(1+|\tau|),(1+2 r)^{\rho}\right\} \leq e^{\rho \widetilde{p}} .
$$

Then,

$$
\begin{aligned}
& |1-\tau+t \tau|^{\left|\widetilde{\theta_{3}}\right|}(|1-\tau||1-t|)^{\left|\theta_{3}-\widetilde{\theta_{3}}\right|}(|\tau||1-t|)^{\left|\widetilde{\epsilon_{3}}\right|}|\tau+t-t \tau|^{\left|\epsilon_{3}-\widetilde{\epsilon_{3}}\right|} \\
& \leq(2(1+|\tau|))^{\left|\theta_{3}+\epsilon_{3}\right|} \leq e^{\rho \widetilde{p}\left|\theta_{3}+\epsilon_{3}\right|} .
\end{aligned}
$$

Moreover, since $(x, y) \in \Delta_{2 r}$, we have

$$
\langle(v+t \tau w)-(v-(1-\tau) t w)\rangle=\langle t(x-y)\rangle<1+2 r \leq e^{\widetilde{p}} .
$$


On the other hand, by Lemma 2.23 , there exists $C=2 \max \left\{(1-\tau)^{2}, \tau^{2}\right\} \geq 1 / 2$ such that

$$
\begin{aligned}
\langle(v+t \tau w, v-t(1-\tau) w, \xi)\rangle^{2} & =1+|v+t \tau w|^{2}+|v-t(1-\tau) w|^{2}+|\xi|^{2} \\
& \geq 1+\frac{1}{C}|v|^{2}+|\xi|^{2} \\
& \geq \frac{1}{2 C}\langle(v, \xi)\rangle^{2}
\end{aligned}
$$

Hence, we have

$$
\langle(v+t \tau w, v-t(1-\tau) w, \xi)\rangle \geq \frac{1}{\sqrt{2} \max \{|1-\tau|,|\tau|\}}\langle(v, \xi)\rangle \geq \frac{1}{e^{1+\widetilde{p}}}\langle(v, \xi)\rangle .
$$

Now, if $k \in \mathbb{N}_{0}$ is as in $(2.16)$, then, by $(0.1)$,

$$
\begin{aligned}
e^{m \omega(v+t \tau w, v-t(1-\tau) w, \xi)} & \leq e^{m L \omega(2(|v|+(|1-\tau|+|\tau|)|w|))} e^{m L \omega(\xi)} e^{m L} \\
& \leq e^{m L^{2} \omega\left(|v|+2^{k}|w|\right)} e^{m L \omega(\xi)} e^{m L^{2}+m L} \\
& \leq e^{m L^{3} \omega(v)} e^{m L^{k+3} \omega(w)} e^{m L \omega(\xi)} e^{m L^{k+3}+\cdots+m L} .
\end{aligned}
$$

We take $\widetilde{n} \geq n$ such that $\left(q \in \mathbb{N}_{0}\right.$ is such that $2^{q} \geq 3 R$ as in the first step)

$$
\widetilde{n} \geq \max \left\{\frac{L^{q}}{\rho}\left(m L^{3}+\lambda L^{2}\right)+1, \frac{L^{q}}{\rho}\left(1+m L+\lambda L^{\widetilde{p}+2}\right)+1\right\} .
$$

Then, by Definition 1.3, using appropriately (0.10) (and Lemma 0.1) we have that there exists $C_{\widetilde{n}}>0$ so that for each $\theta_{3}, \epsilon_{3}, \eta_{3} \in \mathbb{N}_{0}^{d}, \alpha \leq \beta+\gamma \in \mathbb{N}_{0}^{d}$ and $x, y, \xi \in \mathbb{R}^{d}$, by the chain rule

$$
\begin{aligned}
\mid D_{x}^{\theta_{3}} & D_{y}^{\epsilon_{3}}\left(D_{\xi}^{\beta+\gamma-\alpha+\eta_{3}} \omega_{\beta \gamma}\right)(x, y, \xi) \mid \\
\leq & (j+1) \int_{0}^{1}|1-t|^{j} e^{\rho(2 \widetilde{p})\left|\theta_{3}+\epsilon_{3}\right|} C_{\widetilde{n}} \frac{e^{\rho(\widetilde{p}+(1+\widetilde{p}))\left|2 \beta+2 \gamma-\alpha+\theta_{3}+\epsilon_{3}+\eta_{3}\right|}}{\langle(v, \xi)\rangle \rho\left|2 \beta+2 \gamma-\alpha+\theta_{3}+\epsilon_{3}+\eta_{3}\right|} \times \\
& \times e^{16 \widetilde{n} L^{5 \widetilde{p}+4} \rho \varphi^{*}\left(\frac{\left|2 \beta+2 \gamma-\alpha+\theta_{3}+\epsilon_{3}+\eta_{3}\right|}{16 \tilde{n} L^{5 \tilde{p}+4}}\right)} e^{m L^{3} \omega(v)} e^{m L^{k+3} \omega(w)} e^{m L \omega(\xi)} e^{m L^{k+3}+\cdots+m L} d t \\
\leq & C_{\widetilde{n}} e^{16 \widetilde{n} L^{\widetilde{p}+3} \rho \sum_{p=1}^{4 \widetilde{p}+1} L^{p}} e^{m L^{k+3}+\cdots+m L}(j+1)\langle(v, \xi)\rangle^{-\rho|2 \beta+2 \gamma-\alpha|} \times \\
& \times e^{16 \tilde{n} L^{\widetilde{p}+3} \rho \varphi^{*}\left(\frac{\left|2 \beta+2 \gamma-\alpha+\theta_{3}+\epsilon_{3}+\eta_{3}\right|}{16 \widetilde{n} L^{\tilde{p}+3}}\right)} e^{m L^{3} \omega(v)} e^{m L^{k+3} \omega(w)} e^{m L \omega(\xi)} \int_{0}^{1}|1-t|^{j} d t .
\end{aligned}
$$

Now, we proceed similarly as in the first step to obtain an estimate for $\left|D_{x}^{\theta} D_{y}^{\epsilon} Q_{j}(x, y)\right|:$ By Corollary 2.9, there exists $D_{\widetilde{n}}>0$ such that for each 
$\theta_{2}, \epsilon_{2}, \eta_{2}, \alpha \in \mathbb{N}_{0}^{d}$ and $v, \xi \in \mathbb{R}^{d}$,

$$
\begin{aligned}
& \quad\left|D_{x}^{\theta_{2}} D_{y}^{\epsilon_{2}} D_{\xi}^{\alpha+\eta_{2}}\left(\Psi_{j}-\Psi_{j+1}\right)(v, \xi)\right| \\
& \quad \leq D_{\widetilde{n}}\langle(v, \xi)\rangle^{-\rho\left|\alpha+\theta_{2}+\epsilon_{2}+\eta_{2}\right|} e^{16 \tilde{n} L^{\tilde{p}+3} \rho \varphi^{*}\left(\frac{\left|\alpha+\theta_{2}+\epsilon_{2}+\eta_{2}\right|}{16 \tilde{n} L^{\tilde{p}+3}}\right) .}
\end{aligned}
$$

Since $|\beta+\gamma|=j+1$, we obtain

$$
\langle(v, \xi)\rangle^{-\rho\left|\alpha+\theta_{2}+\epsilon_{2}+\eta_{2}\right|}\langle(v, \xi)\rangle^{-\rho|2 \beta+2 \gamma-\alpha|} \leq\left(\langle(v, \xi)\rangle^{-j}\right)^{2 \rho} .
$$

By (0.7), we have (if $|\xi| \geq 1$ )

$$
|\xi|^{\left|\theta_{1}+\epsilon_{1}-\eta_{1}\right|} \leq e^{\lambda L^{\tilde{p}+2} \varphi^{*}\left(\frac{\left|\theta_{1}+\epsilon_{1}-\eta_{1}\right|}{\lambda L^{\tilde{p}+2}}\right)} e^{\lambda L^{\tilde{p}+2} \omega(\xi)},
$$

and from (0.12), for $\lambda>0$ there is $E_{\lambda}>0$ such that

$$
\begin{aligned}
& \frac{(\beta+\gamma) !}{\beta ! \gamma !} \frac{\left(\theta_{1}+\epsilon_{1}\right) !}{\left(\theta_{1}+\epsilon_{1}-\eta_{1}\right) !}|\tau|^{\left|\beta+\epsilon_{2}\right|}|1-\tau|^{\left|\gamma+\theta_{2}\right|} \\
& \quad \leq e^{\rho \widetilde{p}\left|2 \beta+2 \gamma+\theta_{1}+\theta_{2}+\epsilon_{1}+\epsilon_{2}\right|} E_{\lambda} e^{\lambda L^{\tilde{p}+2} \varphi^{*}\left(\frac{\left|\eta_{1}\right|}{\lambda L^{\tilde{p}}+2}\right)} .
\end{aligned}
$$

By Lemma 0.8, it is easy to check that

$$
\begin{aligned}
e^{\rho \widetilde{p}\left|2 \beta+2 \gamma+\theta_{2}+\epsilon_{2}\right|} e^{\rho \widetilde{p}\left|\theta_{1}+\epsilon_{1}\right|} e^{\lambda L^{\tilde{p}+2} \varphi^{*}\left(\frac{\left|\eta_{1}\right|}{\lambda L^{\tilde{p}+2}}\right)} e^{\lambda L^{\tilde{p}+2} \varphi^{*}\left(\frac{\left|\theta_{1}+\epsilon_{1}-\eta_{1}\right|}{\lambda L^{\widetilde{p}+2}}\right)} \times \\
\times e^{16 \widetilde{n} L^{\widetilde{p}+3} \rho \varphi^{*}\left(\frac{\left|\alpha+\theta_{2}+\epsilon_{2}+\eta_{2}\right|}{16 \widetilde{n} L^{p+3}}\right)} e^{16 \widetilde{n} L^{\tilde{p}+3} \rho \varphi^{*}\left(\frac{\left|2 \beta+2 \gamma-\alpha+\theta_{3}+\epsilon_{3}+\eta_{3}\right|}{16 \tilde{n} L^{\tilde{p}+3}}\right)} \\
\leq e^{\lambda L^{2} \sum_{p=1}^{\widetilde{p}} L^{p}} e^{16 \widetilde{n} L^{3} \rho \sum_{p=1}^{\widetilde{p}} L^{p}} e^{\lambda L^{2} \varphi^{*}\left(\frac{|\theta+\epsilon|}{\lambda L^{2}}\right)} e^{n L^{3} \varphi^{*}\left(\frac{|\eta|}{n L^{3}}\right)} \times \\
\quad \times\left(e^{2 \widetilde{n} \varphi^{*}\left(\frac{j}{2 \tilde{n}}\right)}\right)^{2 \rho}\left(e^{2 \widetilde{n} \varphi^{*}\left(\frac{1}{2 \tilde{n}}\right)}\right)^{2 \rho},
\end{aligned}
$$

and also,

$$
e^{\lambda L^{2} \varphi^{*}\left(\frac{|\theta+\epsilon|}{\lambda L^{2}}\right)} e^{n L^{3} \varphi^{*}\left(\frac{|\eta|}{n L^{3}}\right)} e^{2|\theta+\epsilon+\eta|} \leq e^{\lambda \varphi^{*}\left(\frac{|\theta+\epsilon|}{\lambda}\right)} e^{n L \varphi^{*}\left(\frac{|\eta|}{n L}\right)} e^{\lambda L^{2}+\lambda L} e^{n L^{3}+n L^{2}} .
$$

By (2.24) and (2.25), we obtain

$$
\left(e^{2 \widetilde{n} \varphi^{*}\left(\frac{j}{2 n}\right)}\right)^{2 \rho} \leq\left(e^{\widetilde{n} \varphi^{*}\left(\frac{j}{n}\right)}\right)^{2 \rho}\left(e^{-\frac{\tilde{n}-1}{2 L q} \omega(v)}\right)^{2 \rho}\left(e^{-\frac{\tilde{n}-1}{2 L q} \omega(\xi)}\right)^{2 \rho} e^{q(\widetilde{n}-1) 2 \rho} .
$$

From Corollaries 0.23 and 0.20 there are $C_{1}, C_{2}, C_{3}>0$ such that for all $\eta \in \mathbb{N}_{0}^{d}$ and $x, y \in \mathbb{R}^{d}$,

$$
\left|b_{\eta}\right| \leq e^{s C_{1}} e^{-s C_{1} \varphi^{*}\left(\frac{|\eta|}{s C_{1}}\right)}, \quad\left|\frac{1}{G^{s}(y-x)}\right| \leq C_{3}^{s} e^{-s C_{2} \omega(y-x)} .
$$


For the constant $C_{\widetilde{n}}^{\prime}>0$, which is equal to

$$
\begin{aligned}
& C_{\widetilde{n}} D_{\widetilde{n}} e^{16 \widetilde{n} L^{\widetilde{p}+3} \rho \sum_{p=1}^{4 \widetilde{p}+1} L^{p}} e^{16 \widetilde{n} L^{3} \rho \sum_{p=1}^{\widetilde{p}} L^{p}}(j+1) e^{n L^{3}+n L^{2}} e^{q(\widetilde{n}-1) 2 \rho}\left(e^{2 \widetilde{n} \varphi^{*}\left(\frac{1}{2 \widetilde{n}}\right)}\right)^{2 \rho} \times \\
& \times E_{\lambda} e^{\lambda L^{2} \sum_{p=1}^{\widetilde{p}} L^{p}} e^{\lambda L^{2}+\lambda L} e^{m L^{k+3}+\cdots+m L} e^{s C_{1}} C_{3}^{s}\left(\int_{0}^{1}|1-t|^{j} d t\right),
\end{aligned}
$$

we can estimate $\left|D_{x}^{\theta} D_{y}^{\epsilon} Q_{j}(x, y)\right|$ by

$$
\begin{aligned}
& C_{\widetilde{n}}^{\prime} e^{\lambda \varphi^{*}\left(\frac{|\theta+\epsilon|}{\lambda}\right)}\left(\sum_{\eta \in \mathbb{N}_{0}^{d}} e^{-s C_{1} \varphi^{*}\left(\frac{|\eta|}{s C_{1}}\right)} e^{n L \varphi^{*}\left(\frac{|\eta|}{n L}\right)}\right) e^{m L^{3} \omega(v)} e^{-\frac{\widetilde{n}-1}{L q} \rho \omega(v)} e^{m L^{k+3} \omega(w)} \times \\
& \quad \times e^{-s C_{2} \omega(y-x)} \sum_{|\beta+\gamma|=j+1} \sum_{\alpha \leq \beta+\gamma} \frac{1}{\alpha !(\beta+\gamma-\alpha) !} \times \\
& \quad \times \int\left(\langle(v, \xi)\rangle^{-j}\right)^{2 \rho}\left(e^{\widetilde{n} \varphi^{*}\left(\frac{j}{\tilde{n}}\right)}\right)^{2 \rho} e^{\left(m L+\lambda L^{\widetilde{p}+2}-\frac{\tilde{n}-1}{L^{q}} \rho\right) \omega(\xi)} d \xi .
\end{aligned}
$$

Take $s \in \mathbb{N}$ with

$$
s C_{2} \geq m L^{k+3}+\lambda L^{k+2} .
$$

Then, as $\widetilde{n} \geq \frac{L^{q}}{\rho}\left(m L^{3}+\lambda L^{2}\right)+1$, we have, by Lemma 2.22 ,

$$
e^{\left(-\frac{\tilde{n}-1}{L^{q}} \rho+m L^{3}\right) \omega(v)} e^{\left(-s C_{2}+m L^{k+3}\right) \omega(w)} \leq e^{-\lambda \omega(x, y)} e^{\lambda \sum_{p=1}^{k+2} L^{p}} .
$$

Since $\widetilde{n} \geq \frac{L^{q}}{\rho}\left(1+m L+\lambda L^{\widetilde{p}+2}\right)+1$, the integral depending on $\xi$ converges. Taking $n \geq s C_{1}$, the series depending on $\eta$ converges (as in (1.14)).

As the series, by Lemma 0.1 ,

$$
\begin{aligned}
& \sum_{j=1}^{\infty} \sum_{|\beta+\gamma|=j+1} \sum_{\alpha \leq \beta+\gamma} \frac{1}{\alpha !(\beta+\gamma-\alpha) !} \leq \sum_{j=1}^{\infty} \sum_{|\beta+\gamma|=j+1} \frac{1}{(\beta+\gamma) !} 2^{|\beta+\gamma|} \\
& \quad \leq \sum_{j=1}^{\infty} \frac{(2 d)^{j+1}}{(j+1) !} \sum_{|\beta+\gamma|=j+1} 1 \leq \sum_{j=1}^{\infty} \frac{\left(2 d^{2}\right)^{j+1}}{(j+1) !}
\end{aligned}
$$

converges, we can proceed as in the first step and obtain that $(1-\chi) \sum_{j=1}^{\infty} Q_{j} \in$ $\mathcal{S}_{\omega}\left(\mathbb{R}^{2 d}\right)$.

Third step. Let $T_{N}: \mathcal{S}_{\omega}\left(\mathbb{R}^{d}\right) \rightarrow \mathcal{S}_{\omega}\left(\mathbb{R}^{d}\right)$ be the operator with kernel $W_{N}$. Since $A-P=\sum_{N=0}^{\infty} P_{N, \tau}$ converges in $L\left(\mathcal{S}_{\omega}\left(\mathbb{R}^{d}\right), \mathcal{S}_{\omega}^{\prime}\left(\mathbb{R}^{d}\right)\right)$, it follows that $\left(T_{N}\right)$ converges to an operator $T: \mathcal{S}_{\omega}\left(\mathbb{R}^{d}\right) \rightarrow \mathcal{S}_{\omega}\left(\mathbb{R}^{d}\right)$ in $L\left(\mathcal{S}_{\omega}\left(\mathbb{R}^{d}\right), \mathcal{S}_{\omega}^{\prime}\left(\mathbb{R}^{d}\right)\right)$. Indeed, we have shown that

$$
\lim _{N \rightarrow \infty} W_{N}=K_{0}+\sum_{j=1}^{\infty} I_{j}+\sum_{j=1}^{\infty} Q_{j}-K
$$


converges in $\mathcal{S}_{\omega}\left(\mathbb{R}^{2 d}\right)$ as $N \rightarrow \infty$, hence in $\mathcal{S}_{\omega}^{\prime}\left(\mathbb{R}^{2 d}\right)$. By the kernel's theorem, $T_{N}$ converges to some operator $T$ in $L\left(\mathcal{S}_{\omega}\left(\mathbb{R}^{d}\right), \mathcal{S}_{\omega}^{\prime}\left(\mathbb{R}^{d}\right)\right)$.

We show that $T=0$ in $L\left(\mathcal{S}_{\omega}\left(\mathbb{R}^{d}\right), \mathcal{S}_{\omega}^{\prime}\left(\mathbb{R}^{d}\right)\right)$. To this aim, we fix $N \in \mathbb{N}, j_{n} \leq$ $N+1<j_{n+1}$, and we denote $a_{N}:=R e^{\frac{n}{N+1} \varphi^{*}\left(\frac{N+1}{n}\right)}$. According to the support of the derivatives of $\Psi_{N+1}$, we can assume that $2 a_{N} \leq\langle((1-\tau) x+\tau y, \xi)\rangle \leq 3 a_{N}$. For $f, g \in \mathcal{S}_{\omega}\left(\mathbb{R}^{d}\right)$, we have

$$
\left\langle T_{N} f, g\right\rangle=\int T_{N} f(x) g(x) d x=\int\left(\int W_{N}(x, y) f(y) d y\right) g(x) d x .
$$

For fixed $N \in \mathbb{N}$, we show that Fubini's theorem can be used in this integral. In fact, for all $\lambda>0$ there exists $C_{\lambda}>0$ such that

$$
\begin{aligned}
& \quad\left|D_{\xi}^{\alpha} \Psi_{N+1}(v, \xi)\left(\partial_{x}^{\beta} \partial_{y}^{\gamma} D_{\xi}^{\beta+\gamma-\alpha} a\right)(v, v, \xi) f(y) g(x)\right| \\
& \quad \leq C_{\lambda} e^{\varphi^{*}(|\alpha|)} e^{\varphi^{*}(|2 \beta+2 \gamma-\alpha|)} e^{m \omega(v, v, \xi)} e^{-\lambda \omega(y)} e^{-\lambda \omega(x)} .
\end{aligned}
$$

Since $2 a_{N} \leq\langle((1-\tau) x+\tau y, \xi)\rangle \leq 3 a_{N}$, in particular we have $|\xi| \leq 3 a_{N}$ and therefore $1 \leq e^{-\omega(\xi)} e^{\omega\left(3 a_{N}\right)}$. Moreover, by (0.4) and (2.18) (where $k$ and $m^{\prime}$ are as in (2.16) and (2.17)),

$$
\begin{aligned}
m \omega(v, v, \xi) & \leq m L \omega(v)+m L \omega(\xi)+m L \\
& \leq m^{\prime} L \omega(x, y)+m L \omega\left(3 a_{N}\right)+m L^{k+1}+\cdots+m L .
\end{aligned}
$$

Taking $\lambda>m^{\prime} L^{2}+1$ we use (0.3) to get

$$
e^{m^{\prime} L \omega(x, y)} e^{-\lambda \omega(y)} e^{-\lambda \omega(x)} \leq e^{-\omega(x)} e^{-\omega(y)} e^{m^{\prime} L^{2}},
$$

and then we obtain that $(2.26)$ is estimated by a function in $L^{1}\left(\mathbb{R}_{x, y, \xi}^{3 d}\right)$. Therefore, we can use Fubini's theorem in

$$
\begin{aligned}
\left\langle T_{N} f, g\right\rangle= & \int\left(\int \sum_{|\beta+\gamma|=1}^{N} \sum_{0 \neq \alpha \leq \beta+\gamma} \frac{(\beta+\gamma) !}{\beta ! \gamma !} \frac{1}{\alpha !(\beta+\gamma-\alpha) !} \times\right. \\
& \times\left\{\int e^{i(x-y) \cdot \xi} \tau^{|\beta|}(1-\tau)^{|\gamma|}(-1)^{|\gamma|} D_{\xi}^{\alpha} \Psi_{N+1}(v, \xi) \times\right. \\
& \left.\left.\times\left(\partial_{x}^{\beta} \partial_{y}^{\gamma} D_{\xi}^{\beta+\gamma-\alpha} a\right)(v, v, \xi) d \xi\right\} f(y) d y\right) g(x) d x,
\end{aligned}
$$


and integrating by parts in the integrand with formula (1.6) for a suitable power $s \in \mathbb{N}$ to be determined, we have

$$
\begin{aligned}
& e^{i(x-y) \cdot \xi} \frac{1}{G^{s}(\xi)} G^{s}\left(D_{y}\right)\left\{D_{\xi}^{\alpha} \Psi_{N+1}(v, \xi)\left(\partial_{x}^{\beta} \partial_{y}^{\gamma} D_{\xi}^{\beta+\gamma-\alpha} a\right)(v, v, \xi) f(y)\right\} \\
& =e^{i(x-y) \cdot \xi} \frac{1}{G^{s}(\xi)} \sum_{\eta \in \mathbb{N}_{0}^{d}} b_{\eta} \sum_{\eta_{1}+\eta_{2}+\eta_{3}=\eta} \frac{\eta !}{\eta_{1} ! \eta_{2} ! \eta_{3} !} \tau^{\left|\eta_{1}\right|} D_{y}^{\eta_{1}} D_{\xi}^{\alpha} \Psi_{N+1}(v, \xi) \times \\
& \quad \times D_{y}^{\eta_{2}}\left(\partial_{x}^{\beta} \partial_{y}^{\gamma} D_{\xi}^{\beta+\gamma-\alpha} a\right)(v, v, \xi) D_{y}^{\eta_{3}} f(y) .
\end{aligned}
$$

Thus,

$$
\begin{aligned}
\left\langle T_{N} f, g\right\rangle= & \sum_{|\beta+\gamma|=1}^{N} \sum_{0 \neq \alpha \leq \beta+\gamma} \frac{(\beta+\gamma) !}{\beta ! \gamma !} \frac{1}{\alpha !(\beta+\gamma-\alpha) !} \sum_{\eta \in \mathbb{N}_{0}^{d}} b_{\eta} \sum_{\eta_{1}+\eta_{2}+\eta_{3}=\eta} \frac{\eta !}{\eta_{1} ! \eta_{2} ! \eta_{3} !} \times \\
& \times \tau^{\left|\eta_{1}+\beta\right|}(1-\tau)^{|\gamma|}(-1)^{|\gamma|} \iint e^{i(x-y) \cdot \xi} \frac{1}{G^{s}(\xi)} \int D_{y}^{\eta_{1}} D_{\xi}^{\alpha} \Psi_{N+1}(v, \xi) \times \\
& \times D_{y}^{\eta_{2}}\left(\partial_{x}^{\beta} \partial_{y}^{\gamma} D_{\xi}^{\beta+\gamma-\alpha} a\right)(v, v, \xi) D_{y}^{\eta_{3}} f(y) g(x) d y d \xi d x .
\end{aligned}
$$

To estimate $\left|\left\langle T_{N} f, g\right\rangle\right|$, let $\widetilde{p} \in \mathbb{N}_{0}$ be such that $\max \{|1-\tau|, 2|\tau|\} \leq e^{\widetilde{p}}$. By Definition 1.3 and Corollary 2.9, for all $n \in \mathbb{N}$ there exist $C_{n}=C_{4 n L^{\tilde{p}+3}}>0$, $D_{n}=D_{4 n L^{\widetilde{p}+3}}>0$ such that by the chain rule

$$
\begin{aligned}
& \quad\left|D_{y}^{\eta_{2}}\left(\partial_{x}^{\beta} \partial_{y}^{\gamma} D_{\xi}^{\beta+\gamma-\alpha} a\right)(v, v, \xi)\right| \\
& \quad \leq C_{n}\langle(v, \xi)\rangle^{-\rho\left|2 \beta+2 \gamma+\eta_{2}-\alpha\right|}(2|\tau|)^{\left|\eta_{2}\right|} e^{4 n L^{\widetilde{p}+3} \rho \varphi^{*}\left(\frac{\left|2 \beta+2 \gamma+\eta_{2}-\alpha\right|}{4 n L \tilde{p}+3}\right)} e^{m \omega(v, v, \xi)}
\end{aligned}
$$

and

$$
\left|D_{y}^{\eta_{1}} D_{\xi}^{\alpha} \Psi_{N+1}(v, \xi)\right| \leq D_{n}\langle(v, \xi)\rangle^{-\rho\left|\eta_{1}+\alpha\right|} e^{4 n L^{\widetilde{p}+3} \rho \varphi^{*}\left(\frac{\left|\eta_{1}+\alpha\right|}{4 n L \tilde{p}+3}\right)} .
$$

From the choice of $\widetilde{p} \in \mathbb{N}_{0}$,

$$
(2|\tau|)^{\left|\eta_{2}\right|}|\tau|^{\left|\eta_{1}+\beta\right|}|1-\tau|^{|\gamma|} \leq e^{\widetilde{p}\left|\eta_{1}+\eta_{2}+\beta+\gamma\right|} .
$$

We take $0<\ell<n$. Since $f, g \in \mathcal{S}_{\omega}\left(\mathbb{R}^{d}\right)$, there exist $E_{\ell}>0$ (depending on $\ell, \tau, m$ ) and $E^{\prime}>0$ (depending on $\tau, m$ ) such that (where $k$ is as in (2.16))

$$
\begin{aligned}
\left|D_{y}^{\eta_{3}} f(y)\right| & \leq E_{\ell} e^{\ell L^{3} \varphi^{*}\left(\frac{\left|\eta_{3}\right|}{\ell L^{3}}\right)} e^{-\left((m L+L) L^{k+1}+1\right) \omega(y)} \\
|g(x)| & \leq E^{\prime} e^{-\left((m L+L) L^{k+1}+1\right) \omega(x)}
\end{aligned}
$$

By Lemma 0.8 we have

$$
e^{4 n L^{\widetilde{p}+3} \rho \varphi^{*}\left(\frac{\left|\eta_{1}+\alpha\right|}{4 n L^{\tilde{p}+3}}\right)} e^{4 n L^{\widetilde{p}+3} \rho \varphi^{*}\left(\frac{\left|2 \beta+2 \gamma+\eta_{2}-\alpha\right|}{4 n L \widetilde{p}+3}\right)} \leq e^{\ell L^{\widetilde{p}+3} \varphi^{*}\left(\frac{\left|\eta_{1}+\eta_{2}\right|}{\ell L^{\tilde{p}+3}}\right)} e^{2 n \rho \varphi^{*}\left(\frac{|\beta+\gamma|}{n}\right)},
$$


and $\left(\right.$ as $\left.\sum_{\eta_{1}+\eta_{2}+\eta_{3}=\eta} \frac{\eta !}{\eta_{1} ! \eta_{2} ! \eta_{3} !}=3^{|\eta|} \leq e^{2|\eta|}\right)$

$$
\begin{aligned}
& \sum_{\eta_{1}+\eta_{2}+\eta_{3}=\eta} \frac{\eta !}{\eta_{1} ! \eta_{2} ! \eta_{3} !}\left(e^{\widetilde{p}\left|\eta_{1}+\eta_{2}\right|} e^{\ell L^{\widetilde{p}+3} \varphi^{*}\left(\frac{\left|\eta_{1}+\eta_{2}\right|}{\ell L^{\tilde{p}+3}}\right)}\right) e^{\ell L^{3} \varphi^{*}\left(\frac{\left|\eta_{3}\right|}{\ell L^{3}}\right)} \\
& \leq e^{\ell L \sum_{t=1}^{\widetilde{r}+2} L^{t}} e^{\ell L \varphi^{*}\left(\frac{|\eta|}{\ell L}\right)} .
\end{aligned}
$$

On the other hand, since $2 a_{N} \leq\langle(v, \xi)\rangle$ and $1 \leq|\beta+\gamma| \leq N<N+1$, we use that $\varphi^{*}(x) / x$ is increasing to get

$$
\begin{aligned}
\langle(v, \xi)\rangle^{-\rho\left|\eta_{1}+\alpha\right|}\langle(v, \xi)\rangle^{-\rho\left|2 \beta+2 \gamma+\eta_{2}-\alpha\right|} & \leq\langle(v, \xi)\rangle^{-\rho|2 \beta+2 \gamma|} \\
& \leq(2 R)^{-2 \rho|\beta+\gamma|} e^{-2 n \rho \varphi^{*}\left(\frac{|\beta+\gamma|}{n}\right)} .
\end{aligned}
$$

From Corollaries 0.23 and 0.20 there are $C_{1}, C_{2}, C_{3}>0$ such that for all $\eta \in \mathbb{N}_{0}^{d}$, $\xi \in \mathbb{R}^{d}$

$$
\left|b_{\eta}\right| \leq e^{s C_{1}} e^{-s C_{1} \varphi^{*}\left(\frac{|\eta|}{s C_{1}}\right)}, \quad\left|\frac{1}{G^{s}(\xi)}\right| \leq C_{3}^{s} e^{-s C_{2} \omega(\xi)}
$$

Hence, we can estimate $\left|\left\langle T_{N} f, g\right\rangle\right|$ by $\left(\right.$ since $\left.\frac{(\beta+\gamma) !}{\beta ! \gamma !} \leq e^{|\beta+\gamma|}\right)$

$$
\begin{aligned}
& \sum_{|\beta+\gamma|=1}^{N} \sum_{0 \neq \alpha \leq \beta+\gamma}\left(\frac{e^{\tilde{p}+1}}{(2 R)^{2 \rho}}\right)^{|\beta+\gamma|} \frac{1}{\alpha !(\beta+\gamma-\alpha) !} e^{s C_{1}} \times \\
& \quad \times\left(\sum_{\eta \in \mathbb{N}_{0}^{d}} e^{\ell L \varphi^{*}\left(\frac{|\eta|}{\ell L}\right)} e^{-s C_{1} \varphi^{*}\left(\frac{|\eta|}{s C_{1}}\right)}\right) e^{\ell L \sum_{t=1}^{\tilde{p}+2} L^{t}} \int\left(\int C_{3}^{s} e^{-s C_{2} \omega(\xi)} \times\right. \\
& \left.\quad \times\left(\int C_{n} D_{n} E_{\ell} E^{\prime} e^{m \omega(v, v, \xi)} e^{-\left((m L+L) L^{k+1}+1\right)(\omega(y)+\omega(x))} d y\right) d \xi\right) d x .
\end{aligned}
$$

We take $\ell \geq s C_{1}$ to guarantee that the series on $\eta$ is convergent (see (1.14)). We recall that the only factor that depends on $n$ is $C_{n} D_{n}$. We see that if $s C_{2} \geq(m L+L) L^{k+1}+1$, then there exists $C_{k}>0$ such that

$$
\begin{aligned}
& e^{m \omega(v, v, \xi)} e^{-\left((m L+L) L^{k+1}+1\right) \omega(y)} e^{-\left((m L+L) L^{k+1}+1\right) \omega(x)} e^{-s C_{2} \omega(\xi)} \\
& \leq C_{k} e^{-\omega(\langle(v, \xi)\rangle)} e^{-\omega(x)} e^{-\omega(y)} e^{-\omega(\xi)} .
\end{aligned}
$$

In fact, by (0.3), (0.6), and (2.18),

$$
\begin{aligned}
e^{m \omega(v, v, \xi)} e^{\omega(\langle(v, \xi)\rangle)} & \leq e^{(m L+L) \omega(v, \xi)} e^{m L+L} \\
& \leq e^{(m L+L) L^{k} \omega(x, y, \xi)} e^{(m L+L) L^{k}+\cdots+(m L+L) L+m L+L} .
\end{aligned}
$$


Therefore, by (0.4), we obtain (2.27), as

$$
\begin{aligned}
& e^{m \omega(v, v, \xi)} e^{\omega(\langle(v, \xi)\rangle)} e^{\omega(x)} e^{\omega(y)} e^{\omega(\xi)} \\
& \quad \leq e^{\left((m L+L) L^{k+1}+1\right)(\omega(x)+\omega(y)+\omega(\xi))} e^{(m L+L) L^{k+1}+\cdots+(m L+L)} .
\end{aligned}
$$

So, we have

$$
\begin{aligned}
& \iiint_{2 a_{N} \leq\langle(v, \xi)\rangle \leq 3 a_{N}} e^{-\omega(\langle(v, \xi)\rangle)} e^{-\omega(x)-\omega(y)-\omega(\xi)} d y d \xi d x \\
& \leq e^{-\omega\left(2 a_{N}\right)} \iiint_{\mathbb{R}^{3 d}} e^{-\omega(x)-\omega(y)-\omega(\xi)} d y d \xi d x
\end{aligned}
$$

By property $(\gamma)$ of Definition 0.3 , there exists $C>0$ such that $3 \log (t) \leq$ $\omega(t)+C, t \geq 0$. Thus,

$$
e^{-\omega\left(2 a_{N}\right)} \leq\left(2 a_{N}\right)^{-3} e^{C}
$$

By the choice of the sequence $\left(j_{n}\right)_{n}$, we have

$$
e^{n} C_{n} D_{n} \leq a_{N}^{3}
$$

Hence, there exists $C^{\prime}>0$ such that, similarly as in the previous steps,

$$
\begin{aligned}
\left|\left\langle T_{N} f, g\right\rangle\right| & \leq C^{\prime} \sum_{|\beta+\gamma|=1}^{N} \sum_{0 \neq \alpha \leq \beta+\gamma}\left(\frac{e^{\widetilde{p}+1}}{(2 R)^{2 \rho}}\right)^{|\beta+\gamma|} \frac{1}{\alpha !(\beta+\gamma-\alpha) !} \frac{C_{n} D_{n}}{a_{N}^{3}} \\
& \leq \frac{C^{\prime}}{e^{n}} \sum_{l=1}^{N} \frac{1}{l !}\left(\frac{d^{2} e^{\widetilde{p}+1}}{(2 R)^{2 \rho}}\right)^{l} .
\end{aligned}
$$

Since the series converges for $R \geq 1$ large enough (which may depend on $\tau$ ), and since $n \rightarrow \infty$ when $N \rightarrow \infty$, we show that $\left|\left\langle T_{N} f, g\right\rangle\right|$ tends to zero when $N \rightarrow \infty$. It proves that the sequence $\left(T_{N}\right)$ converges to the operator $T=0$ in $L\left(\mathcal{S}_{\omega}\left(\mathbb{R}^{d}\right), \mathcal{S}_{\omega}^{\prime}\left(\mathbb{R}^{d}\right)\right)$, as we wanted.

Following [64, (23.39)], given an amplitude $a(x, y, \xi) \in \mathrm{GA}_{\rho}^{m, \omega}$ and $\tau \in \mathbb{R}$, there exists a symbol $p_{\tau}$ defined by

$$
p_{\tau}(v, \xi):=\mathcal{F}_{w \mapsto \xi} K_{A}(v+\tau w, v-(1-\tau) w),
$$

where $K_{A}$ is the kernel of the operator $A$ given by the amplitude $a$. This symbol is called $\tau$-symbol of the pseudodifferential operator $A$. We write $p$ for the 0 -symbol and, when $\tau=1 / 2$, we write $p_{w}$ for the Weyl symbol. It is unique by the uniqueness of the kernel of $A$. Moreover, we have (see $[64,(23.38)]$ )

$$
K_{A}(x, y)=(2 \pi)^{-d} \mathcal{F}_{\xi \mapsto x-y}^{-1} p_{\tau}((1-\tau) x+\tau y, \xi) .
$$


Definition 2.25. The pseudodifferential operator $P_{\tau}$ associated to the symbol $p_{\tau}$ is called $\tau$-quantization of the operator and satisfies

$$
A=P_{\tau} \text {. }
$$

When $\tau=1 / 2, P_{1 / 2}$ is called Weyl quantization and it is denoted by

$$
P^{w}=p^{w}(x, D) \text {. }
$$

Thus, by Theorem 2.24, we have

$$
\left.p_{\tau}(x, \xi) \sim \sum_{j=0}^{\infty} \sum_{|\beta+\gamma|=j} \frac{1}{\beta ! \gamma !} \tau^{|\beta|}(1-\tau)^{|\gamma|} \partial_{\xi}^{\beta+\gamma}\left(-D_{x}\right)^{\beta} D_{y}^{\gamma} a(x, y, \xi)\right|_{y=x} .
$$

Example 2.26. Let $p \in \mathrm{GS}_{\rho}^{m, \omega}$. Then, we define $a(x, y, \xi):=(2 \pi)^{-d} p\left(\frac{x+y}{2}, \xi\right)$, which belongs to $\mathrm{GA}_{\rho}^{\max \{0, m\}, \omega}$ by Lemma 2.8. Therefore,

$$
A u(x)=\iint e^{i(x-y) \cdot \xi}(2 \pi)^{-d} p\left(\frac{x+y}{2}, \xi\right) u(y) d y d \xi, \quad u \in \mathcal{S}_{\omega}\left(\mathbb{R}^{d}\right) .
$$

On the other hand, by definition of $p_{1 / 2}$, we have, by $(0.34)$,

$$
\begin{aligned}
p_{1 / 2}\left(\frac{x+y}{2}, \xi\right) & =\mathcal{F}_{w \mapsto \xi} K_{A}(x, y)=\mathcal{F}_{w \mapsto \xi}\left(\int e^{i(x-y) \cdot \xi}(2 \pi)^{-d} p\left(\frac{x+y}{2}, \xi\right) d \xi\right) \\
& =\mathcal{F}_{w \mapsto \xi} \mathcal{F}_{\xi \mapsto w}^{-1} p\left(\frac{x+y}{2}, \xi\right)=p\left(\frac{x+y}{2}, \xi\right) .
\end{aligned}
$$

So, in this case, the Weyl symbol coincides with the original global symbol $p$.

Given $b \in \mathrm{GS}_{\rho}^{m, \omega}$, we denote here and below the Weyl quantization $b^{w}(x, D)$ by

$$
b^{w}(x, D) u=(2 \pi)^{-d} \int_{\mathbb{R}^{2 d}} e^{i(x-s) \cdot \xi} b\left(\frac{x+s}{2}, \xi\right) u(s) d s d \xi, \quad x \in \mathbb{R}^{d} .
$$

As a consequence of formula (2.28) and Theorem 2.24, we can describe the precise relation between different quantizations for a given global symbol in terms of equivalence of formal sums as the following result shows (see [64, Theorem 23.3]).

Theorem 2.27. If $a_{\tau_{1}}(x, \xi)$ and $a_{\tau_{2}}(x, \xi)$ are the $\tau_{1}$ and $\tau_{2}$-symbol of the same pseudodifferential operator $A$, then

$$
a_{\tau_{2}}(x, \xi) \sim \sum_{j=0}^{\infty} \sum_{|\alpha|=j} \frac{1}{\alpha !}\left(\tau_{1}-\tau_{2}\right)^{|\alpha|} \partial_{\xi}^{\alpha} D_{x}^{\alpha} a_{\tau_{1}}(x, \xi) .
$$


Proof. By the comments below Theorem 2.24, the pseudodifferential operator $A$ is determined via the $\tau_{1}$-symbol $a_{\tau_{1}}\left(\left(1-\tau_{1}\right) x+\tau_{1} y, \xi\right)$. We denote $e=$ $(1, \ldots, 1) \in \mathbb{N}_{0}^{d}$ and use the fact that

$$
(x+y)^{\alpha}=\sum_{\beta+\gamma=\alpha} \frac{\alpha !}{\beta ! \gamma !} x^{\beta} y^{\gamma}, \quad \alpha \in \mathbb{N}_{0}^{d}, x, y \in \mathbb{R}^{d} .
$$

By formula (2.28), the $\tau_{2}$-symbol of $A$ has the following asymptotic expansion:

$$
\begin{aligned}
a_{\tau_{2}}(x, \xi) & \\
& \sim \sum_{j=0}^{\infty} \sum_{|\beta+\gamma|=j} \frac{(-1)^{|\beta|}}{\beta ! \gamma !} \tau_{2}^{|\beta|}\left(1-\tau_{2}\right)^{|\gamma|} \partial_{\xi}^{\beta+\gamma} D_{x}^{\beta} D_{y}^{\gamma}\left(\left.a_{\tau_{1}}\left(\left(1-\tau_{1}\right) x+\tau_{1} y, \xi\right)\right|_{y=x}\right) \\
& =\sum_{j=0}^{\infty} \sum_{|\alpha|=j}\left(\sum_{\beta+\gamma=\alpha} \frac{1}{\beta ! \gamma !}\left(-\tau_{2}\left(1-\tau_{1}\right)\right)^{|\beta|}\left(\left(1-\tau_{2}\right) \tau_{1}\right)^{|\gamma|}\right) \partial_{\xi}^{\alpha} D_{x}^{\alpha} a_{\tau_{1}}(x, \xi) \\
& =\sum_{j=0}^{\infty} \sum_{|\alpha|=j} \frac{1}{\alpha !}\left(-\tau_{2}\left(1-\tau_{1}\right)+\left(1-\tau_{2}\right) \tau_{1}\right)^{|\alpha|} \partial_{\xi}^{\alpha} D_{x}^{\alpha} a_{\tau_{1}}(x, \xi) \\
& =\sum_{j=0}^{\infty} \sum_{|\alpha|=j} \frac{1}{\alpha !}\left(\tau_{1}-\tau_{2}\right)^{|\alpha|} \partial_{\xi}^{\alpha} D_{x}^{\alpha} a_{\tau_{1}}(x, \xi) .
\end{aligned}
$$

\subsection{The transposition and composition of operators}

Given a symbol $a(x, \xi)$, let $A$ be the pseudodifferential operator associated to the amplitude $a((1-\tau) x+\tau y, \xi)$. By Proposition 1.18 (see [33, Theorem 2.5]) we obtain that the transpose $A^{t}$ is associated to the amplitude $a((1-\tau) y+$ $\tau x,-\xi)$. Hence, if $a_{\tau}(x, \xi)$ is the $\tau$-symbol of $A$, then the $(1-\tau)$-symbol of $A^{t}$ is given by

$$
a_{1-\tau}^{t}((1-\tau) x+\tau y, \xi):=a_{\tau}((1-\tau) y+\tau x,-\xi) .
$$

This formula is equivalent to $a_{\tau}^{t}(\tau x+(1-\tau) y, \xi)=a_{1-\tau}(\tau y+(1-\tau) x,-\xi)$. As a consequence of $(2.30)$, when $y=x$ we have $a_{\tau}^{t}(x, \xi)=a_{1-\tau}(x,-\xi)$. On the other hand, for $\tau=0, a_{1}^{t}(y,-\xi)$ coincides with $a_{0}(x, \xi)$, and for $\tau=1 / 2$, we have $a_{w}^{t}\left(\frac{x+y}{2}, \xi\right)=a_{w}\left(\frac{x+y}{2},-\xi\right)$.

By Theorem 2.24, the transpose of a pseudodifferential operator (restricted to $\mathcal{S}_{\omega}\left(\mathbb{R}^{d}\right)$ ) can be described, modulus an $\omega$-regularizing operator, by another pseudodifferential operator with a precise relation between their $\tau$-symbols: 
Theorem 2.28. Let $A$ be the pseudodifferential operator with $\tau$-symbol $a_{\tau}(x, \xi)$ in $\mathrm{GS}_{\rho}^{m, \omega}$. Then its transpose restricted to $\mathcal{S}_{\omega}\left(\mathbb{R}^{d}\right)$ can be decomposed as $A^{t}=P+R$, where $R$ is an w-regularizing operator and $P$ is the pseudodifferential operator associated to the symbol given by

$$
p(x, \xi) \sim a_{\tau}^{t}(x, \xi):=\sum_{j=0}^{\infty} \sum_{|\alpha|=j} \frac{1}{\alpha !}(1-2 \tau)^{|\alpha|} \partial_{\xi}^{\alpha} D_{x}^{\alpha} a_{\tau}(x,-\xi) .
$$

Proof. First, by Proposition 2.13 it follows that the formal sum above belongs to $\mathrm{FGS}_{\rho}^{m, \omega}$. By assumption we have that $A^{t}$ has the $(1-\tau)$-symbol $a_{1-\tau}^{t}(x, \xi)$ given by formula (2.30) with $y=x$. Moreover, from Theorem 2.27, the $\tau$ symbol of $A^{t}$ satisfies

$$
\begin{aligned}
a_{\tau}^{t}(x, \xi) & \sim \sum_{j=0}^{\infty} \sum_{|\alpha|=j} \frac{1}{\alpha !}(1-2 \tau)^{|\alpha|} \partial_{\xi}^{\alpha} D_{x}^{\alpha} a_{1-\tau}^{t}(x, \xi) \\
& =\sum_{j=0}^{\infty} \sum_{|\alpha|=j} \frac{1}{\alpha !}(1-2 \tau)^{|\alpha|} \partial_{\xi}^{\alpha} D_{x}^{\alpha} a_{\tau}(x,-\xi) .
\end{aligned}
$$

In what follows, we deal with the composition of two pseudodifferential operators given by arbitrary quantizations. In fact, given such quantizations of two pseudodifferential operators, we can describe the formal sum of the $\tau$-symbol of the resulting composition:

Theorem 2.29. Let $a_{\tau_{1}}(x, \xi) \in \mathrm{GS}_{\rho}^{m_{1}, \omega}$ be the $\tau_{1}$-symbol of $A$ and $b_{\tau_{2}}(x, \xi) \in$ $\mathrm{GS}_{\rho}^{m_{2}, \omega}$ be the $\tau_{2}$-symbol of $B$. The $\tau$-symbol $c_{\tau}(x, \xi) \in \mathrm{GS}_{\rho}^{m_{1}+m_{2}, \omega}$ of $A \circ B$ has the asymptotic expansion

$$
\sum_{j=0}^{\infty} \sum_{\substack{\left|\alpha+\beta-\alpha_{1}-\alpha_{2}\right|=j \\ \alpha+\beta=\gamma+\delta}} c_{\alpha \beta \gamma \delta \alpha_{1} \alpha_{2}} \partial_{\xi}^{\gamma} D_{x}^{\alpha} a_{\tau_{1}}(x, \xi) \cdot \partial_{\xi}^{\delta} D_{x}^{\beta} b_{\tau_{2}}(x, \xi),
$$

where the coefficients $c_{\alpha \beta \gamma \delta \alpha_{1} \alpha_{2}}$ equal

$$
\begin{aligned}
& \frac{(2 \pi)^{d}}{\gamma ! \delta !} \sum_{k, l=0}^{\infty} \sum_{\substack{\left|\alpha_{1}\right|=k \\
\left|\alpha_{2}\right|=l}}(-1)^{\left|\alpha-\alpha_{1}+\alpha_{2}\right|}\left(\begin{array}{c}
\alpha+\beta-\alpha_{1}-\alpha_{2} \\
\alpha-\alpha_{1}
\end{array}\right)\left(\begin{array}{c}
\gamma \\
\alpha_{1}
\end{array}\right)\left(\begin{array}{c}
\delta \\
\alpha_{2}
\end{array}\right) \times \\
& \quad \times \tau^{\left|\alpha-\alpha_{1}\right|}(1-\tau)^{\left|\beta-\alpha_{2}\right|} \tau_{1}^{\left|\alpha_{1}\right|}\left(1-\tau_{2}\right)^{\left|\alpha_{2}\right|} .
\end{aligned}
$$


Proof. We first assume $\tau_{1}=0$ and $\tau_{2}=1$. Then, for $u \in \mathcal{S}_{\omega}\left(\mathbb{R}^{d}\right)$,

$$
(A \circ B) u(x)=\int e^{i x \cdot \xi} a_{0}(x, \xi) \widehat{B u}(\xi) d \xi, \quad x \in \mathbb{R}^{d}
$$

We see that $B u(x)=\widehat{I}(-x)$, where $I(\xi)=\int e^{-i y \cdot \xi} b_{1}(y, \xi) u(y) d y$. Indeed,

$$
\begin{aligned}
\widehat{I}(-x) & =\int e^{i x \cdot \xi} I(\xi) d \xi \\
& =\int e^{i x \cdot \xi} \int e^{-i y \cdot \xi} b_{1}(y, \xi) u(y) d y d \xi \\
& =\int\left(\int e^{i(x-y) \cdot \xi} b_{1}(y, \xi) u(y) d y\right) d \xi=B u(x) .
\end{aligned}
$$

Hence, by $(0.34), \widehat{B u}(\xi)=(2 \pi)^{d} I(\xi)$ and

$$
(A \circ B) u(x)=\iint e^{i(x-y) \cdot \xi} c(x, y, \xi) u(y) d y d \xi, \quad x \in \mathbb{R}^{d},
$$

where $c(x, y, \xi)=(2 \pi)^{d} a_{0}(x, \xi) b_{1}(y, \xi)$ is an amplitude in $\mathrm{GA}_{\rho}^{m_{1}+m_{2}, \omega}$. By formula (2.28) (see Theorem 2.24), the $\tau$-symbol $c_{\tau}(x, \xi)$ has the asymptotic expansion:

$$
\begin{aligned}
& \left.(2 \pi)^{d} \sum_{j=0}^{\infty} \sum_{|\beta+\gamma|=j} \frac{(-1)^{|\beta|}}{\beta ! \gamma !} \tau^{|\beta|}(1-\tau)^{|\gamma|} \partial_{\xi}^{\beta+\gamma} D_{x}^{\beta} D_{y}^{\gamma}\left(a_{0}(x, \xi) b_{1}(y, \xi)\right)\right|_{y=x} \\
& =(2 \pi)^{d} \sum_{j=0}^{\infty} \sum_{\substack{|\beta+\gamma|=j \\
\delta+\epsilon=\beta+\gamma}} \frac{(-1)^{|\beta|}(\beta+\gamma) !}{\delta ! \epsilon ! \beta ! \gamma !} \tau^{|\beta|}(1-\tau)^{|\gamma|} \partial_{\xi}^{\delta} D_{x}^{\beta} a_{0}(x, \xi) \cdot \partial_{\xi}^{\epsilon} D_{x}^{\gamma} b_{1}(x, \xi)
\end{aligned}
$$

Now, we treat the general case, by making use of (2.33). By Theorem 2.27, we have

$$
\begin{aligned}
a_{0}(x, \xi) & \sim \sum_{j_{1}=0}^{\infty} \sum_{\left|\alpha_{1}\right|=j_{1}} \frac{1}{\alpha_{1} !} \tau_{1}^{\left|\alpha_{1}\right|} \partial_{\xi}^{\alpha_{1}} D_{x}^{\alpha_{1}} a_{\tau_{1}}(x, \xi) \\
b_{1}(x, \xi) & \sim \sum_{j_{2}=0}^{\infty} \sum_{\left|\alpha_{2}\right|=j_{2}} \frac{(-1)^{\left|\alpha_{2}\right|}}{\alpha_{2} !}\left(1-\tau_{2}\right)^{\left|\alpha_{2}\right|} \partial_{\xi}^{\alpha_{2}} D_{x}^{\alpha_{2}} b_{\tau_{2}}(x, \xi),
\end{aligned}
$$


so we reformulate $(2.33)$ as

$$
\begin{aligned}
c_{\tau}(x, \xi) \sim & (2 \pi)^{d} \sum_{j=0}^{\infty} \sum_{\substack{|\beta+\gamma|=j \\
\delta+\epsilon=\beta+\gamma}} \frac{(-1)^{|\beta|}(\beta+\gamma) !}{\delta ! \epsilon ! \beta ! \gamma !} \tau^{|\beta|}(1-\tau)^{|\gamma|} \times \\
& \times \partial_{\xi}^{\delta} D_{x}^{\beta}\left(\sum_{j_{1}=0}^{\infty} \sum_{\left|\alpha_{1}\right|=j_{1}} \frac{1}{\alpha_{1} !} \tau_{1}^{\left|\alpha_{1}\right|} \partial_{\xi}^{\alpha_{1}} D_{x}^{\alpha_{1}} a_{\tau_{1}}(x, \xi)\right) \times \\
& \times \partial_{\xi}^{\epsilon} D_{x}^{\gamma}\left(\sum_{j_{2}=0}^{\infty} \sum_{\left|\alpha_{2}\right|=j_{2}} \frac{(-1)^{\left|\alpha_{2}\right|}}{\alpha_{2} !}\left(1-\tau_{2}\right)^{\left|\alpha_{2}\right|} \partial_{\xi}^{\alpha_{2}} D_{x}^{\alpha_{2}} b_{\tau_{2}}(x, \xi)\right) .
\end{aligned}
$$

By the change of variables $\gamma^{\prime}=\alpha_{1}+\delta, \alpha^{\prime}=\alpha_{1}+\beta, \delta^{\prime}=\alpha_{2}+\epsilon, \beta^{\prime}=\alpha_{2}+\gamma$, it follows that

$$
\begin{aligned}
c_{\tau}(x, \xi) \sim & (2 \pi)^{d} \sum_{\substack { j=0 \\
\begin{subarray}{c}{\alpha^{\prime}+\beta^{\prime}-\alpha_{1}-\alpha_{2} \mid=j \\
\alpha^{\prime}+\beta^{\prime}=\delta^{\prime}+\gamma^{\prime}{ j = 0 \\
\begin{subarray} { c } { \alpha ^ { \prime } + \beta ^ { \prime } - \alpha _ { 1 } - \alpha _ { 2 } | = j \\
\alpha ^ { \prime } + \beta ^ { \prime } = \delta ^ { \prime } + \gamma ^ { \prime } } }\end{subarray}}^{\infty} \frac{1}{\gamma^{\prime} ! \delta^{\prime} !} \partial_{\xi}^{\gamma^{\prime}} D_{x}^{\alpha^{\prime}} a_{\tau_{1}}(x, \xi) \partial_{\xi}^{\delta^{\prime}} D_{x}^{\beta^{\prime}} b_{\tau_{2}}(x, \xi) \times \\
& \times \sum_{k, l=0}^{\infty} \sum_{\substack{\left|\alpha_{1}\right|=k \\
\left|\alpha_{2}\right|=l}}(-1)^{\left|\alpha^{\prime}-\alpha_{1}+\alpha_{2}\right|} \frac{\left(\alpha^{\prime}+\beta^{\prime}-\alpha_{1}-\alpha_{2}\right) !}{\left(\alpha^{\prime}-\alpha_{1}\right) !\left(\beta^{\prime}-\alpha_{2}\right) !} \frac{\gamma^{\prime} !}{\alpha_{1} !\left(\gamma^{\prime}-\alpha_{1}\right) !} \times \\
& \times \frac{\delta^{\prime} !}{\alpha_{2} !\left(\delta^{\prime}-\alpha_{2}\right) !} \tau^{\left|\alpha^{\prime}-\alpha_{1}\right|}(1-\tau)^{\left|\beta^{\prime}-\alpha_{2}\right|} \tau_{1}^{\left|\alpha_{1}\right|}\left(1-\tau_{2}\right)^{\left|\alpha_{2}\right|},
\end{aligned}
$$

which concludes the proof.

The coefficients appearing in (2.31), which depend on the quantizations, are sometimes simplified. For instance, when $\tau_{1}=\tau_{2}=\tau$. As an immediate consequence we obtain [64, Problem 23.2] adapted to our context. First, we need a lemma (see [9, Theorem 5.5]).

Lemma 2.30. For all $\beta, \gamma, \epsilon \in \mathbb{N}_{0}^{d}$ such that $\epsilon \leq \beta+\gamma$, it holds

$$
\frac{(\beta+\gamma) !}{(\beta+\gamma-\epsilon) ! \epsilon !} \frac{1}{\beta ! \gamma !}=\sum_{\substack{0 \leq \delta \leq \beta \\ \beta-\epsilon \leq \delta \leq \beta-\epsilon+\gamma}} \frac{1}{(\beta-\delta) !(\beta-\epsilon+\gamma-\delta) ! \delta !(\delta-\beta+\epsilon) !}
$$

Example 2.31. Given two pseudodifferential operators $A, B: \mathcal{S}_{\omega}\left(\mathbb{R}^{d}\right) \rightarrow$ $\mathcal{S}_{\omega}\left(\mathbb{R}^{d}\right)$, the $\tau$-symbol of the composition operator $C=A \circ B$ is given by $c_{\tau}(x, \xi) \sim(2 \pi)^{d} \sum_{j=0}^{\infty} \sum_{|\beta+\gamma|=j} \frac{(-1)^{|\beta|}}{\beta ! \gamma !} \tau^{|\beta|}(1-\tau)^{|\gamma|}\left(\partial_{\xi}^{\gamma} D_{x}^{\beta} a_{\tau}(x, \xi)\right)\left(\partial_{\xi}^{\beta} D_{x}^{\gamma} b_{\tau}(x, \xi)\right)$. 
Proof. Formula (2.33) states that $c_{\tau}(x, \xi)$ is equivalent to (since $\delta=\beta+\gamma-\epsilon$ )

$$
\begin{aligned}
c_{\tau}(x, \xi) \sim & (2 \pi)^{d} \sum_{j=0}^{\infty} \sum_{|\beta+\gamma|=j}(-1)^{|\beta|} \tau^{|\beta|}(1-\tau)^{|\gamma|} \sum_{\epsilon \leq \beta+\gamma} \frac{(\beta+\gamma) !}{(\beta+\gamma-\epsilon) ! \epsilon !} \frac{1}{\beta ! \gamma !} \times \\
& \times \partial_{\xi}^{\beta+\gamma-\epsilon} D_{x}^{\beta} a_{0}(x, \xi) \cdot \partial_{\xi}^{\epsilon} D_{x}^{\gamma} b_{1}(x, \xi) .
\end{aligned}
$$

Moreover, by Lemma 2.30,

$$
\begin{aligned}
c_{\tau}(x, \xi) \sim & (2 \pi)^{d} \sum_{j=0}^{\infty} \sum_{|\beta+\gamma|=j}(-1)^{|\beta|} \tau^{|\beta|}(1-\tau)^{|\gamma|} \times \\
& \times \sum_{\epsilon \leq \beta+\gamma} \sum_{\substack{0 \leq \delta \leq \beta \\
\beta-\epsilon \leq \delta \leq \beta-\epsilon+\gamma}} \frac{1}{(\beta-\delta) !(\beta-\epsilon+\gamma-\delta) ! \delta !(\delta-\beta+\epsilon) !} \times \\
& \times \partial_{\xi}^{\beta+\gamma-\epsilon} D_{x}^{\beta} a_{0}(x, \xi) \cdot \partial_{\xi}^{\epsilon} D_{x}^{\gamma} b_{1}(x, \xi) .
\end{aligned}
$$

We put $\mu=\beta-\delta, \nu=\beta-\epsilon+\gamma-\delta$, and $\theta=\delta-\beta+\epsilon$. Therefore,

$$
\begin{aligned}
c_{\tau}(x, \xi) \sim & (2 \pi)^{d} \sum_{j=0}^{\infty} \sum_{|\nu+\theta+\mu+\delta|=j} \frac{(-1)^{|\mu+\delta|}}{\mu ! \nu ! \delta ! \theta !} \tau^{|\mu+\delta|}(1-\tau)^{|\nu+\theta|} \times \\
& \times \partial_{\xi}^{\nu+\delta} D_{x}^{\mu+\delta} a_{0}(x, \xi) \cdot \partial_{\xi}^{\mu+\theta} D_{x}^{\nu+\theta} b_{1}(x, \xi),
\end{aligned}
$$

and taking $j=j_{1}+j_{2}+j_{3}, j_{1}, j_{2}, j_{3} \in \mathbb{N}_{0}$, we have that $c_{\tau}(x, \xi)$ is equivalent to

$$
\begin{aligned}
(2 \pi)^{d} & \sum_{j_{1}=0}^{\infty} \sum_{|\nu+\mu|=j_{1}} \frac{(-1)^{|\mu|}}{\mu ! \nu !} \tau^{|\mu|}(1-\tau)^{|\nu|} \times \\
& \times \partial_{\xi}^{\nu} D_{x}^{\mu}\left(\sum_{j_{2}=0}^{\infty} \sum_{|\delta|=j_{2}} \frac{(-1)^{|\delta|}}{\delta !} \tau^{|\delta|} \partial_{\xi}^{\delta} D_{x}^{\delta} a_{0}(x, \xi)\right) \times \\
& \times \partial_{\xi}^{\mu} D_{x}^{\nu}\left(\sum_{j_{3}=0}^{\infty} \sum_{|\theta|=j_{3}} \frac{1}{\theta !}(1-\tau)^{|\theta|} \partial_{\xi}^{\theta} D_{x}^{\theta} b_{1}(x, \xi)\right) .
\end{aligned}
$$

We get the result since Theorem 2.27 gives

$$
\begin{aligned}
& a_{\tau}(x, \xi) \sim \sum_{k=0}^{\infty} \sum_{|\delta|=k} \frac{(-1)^{|\delta|}}{\delta !} \tau^{|\delta|} \partial_{\xi}^{\delta} D_{x}^{\delta} a_{0}(x, \xi) \\
& b_{\tau}(x, \xi) \sim \sum_{k=0}^{\infty} \sum_{|\theta|=k} \frac{1}{\theta !}(1-\tau)^{|\theta|} \partial_{\xi}^{\theta} D_{x}^{\theta} b_{1}(x, \xi) .
\end{aligned}
$$


Corollary 2.32. Given two pseudodifferential operators $A, B: \mathcal{S}_{\omega}\left(\mathbb{R}^{d}\right) \rightarrow$ $\mathcal{S}_{\omega}\left(\mathbb{R}^{d}\right)$, the Weyl symbol of the composition operator $C=A \circ B$ is given by

$$
c_{w}(x, \xi) \sim(2 \pi)^{d} \sum_{j=0}^{\infty} \sum_{|\beta+\gamma|=j} \frac{(-1)^{|\beta|}}{\gamma ! \beta !} 2^{-|\beta+\gamma|}\left(\partial_{\xi}^{\gamma} D_{x}^{\beta} a_{w}(x, \xi)\right)\left(\partial_{\xi}^{\beta} D_{x}^{\gamma} b_{w}(x, \xi)\right) .
$$

In particular, we obtain [6, Theorem 5.7].

Corollary 2.33. Let $A, B: \mathcal{S}_{\omega}\left(\mathbb{R}^{d}\right) \rightarrow \mathcal{S}_{\omega}\left(\mathbb{R}^{d}\right)$ be two pseudodifferential operators with global symbols $a(x, \xi) \in \mathrm{GS}_{\rho}^{m_{1}, \omega}$ and $b(x, \xi) \in \mathrm{GS}_{\rho}^{m_{2}, \omega}$. Then, the global symbol $c(x, \xi) \in \mathrm{GS}_{\rho}^{m_{1}+m_{2}, \omega}$ associated to $C=A \circ B: \mathcal{S}_{\omega}\left(\mathbb{R}^{d}\right) \rightarrow \mathcal{S}_{\omega}\left(\mathbb{R}^{d}\right)$ satisfies

$$
c(x, \xi) \sim(2 \pi)^{d}(a(x, \xi) \circ b(x, \xi))=(2 \pi)^{d} \sum_{j=0}^{\infty} \sum_{|\gamma|=j} \frac{1}{\gamma !}\left(\partial_{\xi}^{\gamma} a(x, \xi)\right)\left(D_{x}^{\gamma} b(x, \xi)\right) .
$$

We finally show [64, Problem 23.1]:

Example 2.34. The global symbol $p(x, \xi)$ of a pseudodifferential operator $P$ can be expressed in terms of $P$ via the formula

$$
p(x, \xi)=(2 \pi)^{-d} e^{-i x \cdot \xi} P\left(e^{i(\cdot) \cdot \xi}\right)(x) .
$$

Proof. By Theorem 2.28, we deduce that if $P$ is the pseudodifferential operator given by the symbol $p(x, \xi)$, the transpose operator is given by the amplitude $p(y,-\xi)$ (see $(2.30))$. So, for $f \in \mathcal{S}_{\omega}\left(\mathbb{R}^{d}\right)$ we have

$$
\left(P^{t} f\right)(x)=\int e^{i x \cdot \xi}\left(\int e^{-i y \cdot \xi} p(y,-\xi) f(y) d y\right) d \xi
$$

By an integration by parts with the ultradifferential operator $G^{n}(D)$, for $n \in$ $\mathbb{N}_{0}$ large enough, with the formula (similar to (1.6))

$$
e^{-i y \cdot \xi}=\frac{1}{G^{n}(\xi)} G^{n}\left(-D_{y}\right) e^{-i y \cdot \xi}
$$

one can show that

$$
I(\xi)=\int e^{-i y \cdot \xi} p(y,-\xi) f(y) d y, \quad f \in \mathcal{S}_{\omega}\left(\mathbb{R}^{d}\right)
$$


belongs to $L^{1}\left(\mathbb{R}^{d}\right)$. In fact, the integration by parts yields

$$
\begin{aligned}
e^{-i y \cdot \xi} & \frac{1}{G^{n}(\xi)} G^{n}\left(D_{y}\right)\{p(y,-\xi) f(y)\} \\
& =e^{-i y \cdot \xi} \frac{1}{G^{n}(\xi)} \sum_{\eta \in \mathbb{N}_{0}^{d}} b_{\eta} \sum_{\eta_{1}+\eta_{2}=\eta} \frac{\eta !}{\eta_{1} ! \eta_{2} !} D_{y}^{\eta_{1}} p(y,-\xi) D_{y}^{\eta_{2}} f(y) .
\end{aligned}
$$

Therefore,

$$
I(\xi)=\frac{1}{G^{n}(\xi)} \sum_{\eta \in \mathbb{N}_{0}^{d}} b_{\eta} \sum_{\eta_{1}+\eta_{2}=\eta} \frac{\eta !}{\eta_{1} ! \eta_{2} !} \int e^{-i y \cdot \xi} D_{y}^{\eta_{1}} p(y,-\xi) D_{y}^{\eta_{2}} f(y) d y
$$

From Corollaries 0.23 and 0.20 there are $C_{1}, C_{2}, C_{3}>0$ (depending only on $G)$ such that for all $\eta \in \mathbb{N}_{0}^{d}$ and $\xi \in \mathbb{R}^{d}$

$$
\left|b_{\eta}\right| \leq e^{n C_{1}} e^{-n C_{1} \varphi^{*}\left(\frac{|\eta|}{n C_{1}}\right)}, \quad\left|\frac{1}{G^{n}(\xi)}\right| \leq C_{3}^{n} e^{-n C_{2} \omega(\xi)} .
$$

Assume $m \geq 0$ without losing generality. For all $\lambda>0$, there exist $C_{\lambda}>0$ (see (1.11)) and $D_{\lambda}>0$ such that for all $\eta_{1}, \eta_{2} \in \mathbb{N}_{0}^{d}$ and $y, \xi \in \mathbb{R}^{d}$,

$$
\begin{aligned}
\left|D_{y}^{\eta_{1}} p(y,-\xi)\right| & \leq C_{\lambda} e^{\lambda L^{2} \varphi^{*}\left(\frac{\left|\eta_{1}\right|}{\lambda L^{2}}\right)} e^{m \omega(y,-\xi)} \\
\left|D_{y}^{\eta_{2}} f(y)\right| & \leq D_{\lambda} e^{\lambda L^{2} \varphi^{*}\left(\frac{\left|\eta_{2}\right|}{\lambda L^{2}}\right)} e^{-(m L+1) \omega(y)}
\end{aligned}
$$

Therefore,

$$
\begin{aligned}
|I(\xi)| \leq & e^{n C_{1}} C_{3}^{n} e^{-n C_{2} \omega(\xi)} \sum_{\eta \in \mathbb{N}_{0}^{d}} e^{-n C_{1} \varphi^{*}\left(\frac{|\eta|}{n C_{1}}\right)} \sum_{\eta_{1}+\eta_{2}=\eta} \frac{\eta !}{\eta_{1} ! \eta_{2} !} \times \\
& \times C_{\lambda} D_{\lambda} e^{\lambda L^{2} \varphi^{*}\left(\frac{\left|\eta_{1}\right|}{\lambda L^{2}}\right)} e^{\lambda L^{2} \varphi^{*}\left(\frac{\left|\eta_{2}\right|}{\lambda L^{2}}\right)} \int e^{m \omega(y,-\xi)} e^{-(m L+1) \omega(y)} d y .
\end{aligned}
$$

We take $n \in \mathbb{N}_{0}$ such that $n C_{2} \geq m L+1$. In particular, by $(0.3)$,

$$
e^{-n C_{2} \omega(\xi)} e^{m \omega(y,-\xi)} e^{-(m L+1) \omega(y)} \leq e^{-\omega(\xi)} e^{-\omega(y)} e^{m L}
$$

This implies the convergence of the integral in (2.34). On the other hand, by Lemma 0.8

$$
\sum_{\eta_{1}+\eta_{2}=\eta} \frac{\eta !}{\eta_{1} ! \eta_{2} !} e^{\lambda L^{2} \varphi^{*}\left(\frac{\left|\eta_{1}\right|}{\lambda L^{2}}\right)} e^{\lambda L^{2} \varphi^{*}\left(\frac{\left|\eta_{2}\right|}{\lambda L^{2}}\right)} \leq e^{\lambda L \varphi^{*}\left(\frac{|\eta|}{\lambda L}\right)} e^{\lambda L^{2}}
$$


so it is enough to fix $\lambda \geq n C_{1}$ to obtain that the series depending on $\eta \in \mathbb{N}_{0}^{d}$ in (2.34) converges (see (1.14)). Hence, there exists $C^{\prime}>0$ that depends on $\lambda>0$ such that $|I(\xi)| \leq C^{\prime} e^{-\omega(\xi)}, \xi \in \mathbb{R}^{d}$.

Thus we have $\widehat{I}(-x)=\left(P^{t} f\right)(x) \in \mathcal{S}_{\omega}\left(\mathbb{R}^{d}\right)$. Hence $\widehat{I} \in \mathcal{S}_{\omega}\left(\mathbb{R}^{d}\right)$. Therefore, as $e^{i x \cdot \xi} \in \mathcal{E}_{(\omega)}\left(\mathbb{R}^{2 d}\right) \subseteq \mathcal{S}_{\omega}^{\prime}\left(\mathbb{R}^{2 d}\right)$, for $f \in \mathcal{S}_{\omega}\left(\mathbb{R}^{d}\right)$, we have by $(0.34)$

$$
\begin{aligned}
\left\langle P\left(e^{i x \cdot \xi}\right), f\right\rangle & =\left\langle e^{i x \cdot \xi}, P^{t} f\right\rangle=\int e^{i x \cdot \xi} \widehat{I}(-x) d x \\
& =(2 \pi)^{d} I(-\xi)=(2 \pi)^{d} \int e^{i x \cdot \xi} p(x, \xi) f(x) d x .
\end{aligned}
$$

This shows the result. 


\section{Chapter 3}

\section{Parametrices}

The notion of hypoellipticity comes from the problem of determining whether a distribution solution to the partial differential equation $P u=f$, where $f$ is a smooth function, is a classical solution or not. The authors in [33] provide adequate conditions for the construction of a (left) parametrix for their symbols, which guarantee the hypoellipticity in the desired class in [32]. For the operators defined in [58], the corresponding construction of parametrices is done in [25].

We develop the method of the parametrix for the class of operators introduced in Chapter 1. That is, we obtain sufficient conditions for the symbol of a pseudodifferential operator to have a parametrix and, in particular, to be $\omega$-regular in the sense of Shubin [64]; see the definition of $\omega$-regularity at the beginning of Section 3.1 and Corollary 3.4. Given a pseudodifferential operator $P$, we say that another pseudodifferential operator $Q$ is a left parametrix for $P$ if

$$
Q \circ P=I+R,
$$

where $I$ is the identity operator and $R$ is an $\omega$-regularizing operator.

The conditions imposed to symbols to construct parametrices motivate the definition of a wave front set given in terms of Weyl quantizations for $\mathcal{S}_{\omega}^{\prime}\left(\mathbb{R}^{d}\right)$ in Chapter 4, called Weyl wave front set. 
We also give examples of symbols with prescribed exponential growth which satisfy the conditions to admit a parametrix. However, we need to take weight functions $\omega$ bounded from above by the Gevrey weight function $\sigma(t)=t^{1 / 2}$.

Finally, inspired by Boggiatto, Buzano, and Rodino [9], we show that some type of symbols, which in addition satisfies the sufficient conditions of the existence of parametrices, called $\omega$-hypoelliptic symbols, are still $\omega$-hypoelliptic under a change of quantization. Moreover, we compare the notions of $\omega$ regularity and $\omega$-hypoellipticity following the ideas of [13].

The following results appear in [4].

\subsection{Global regularity}

We say that a pseudodifferential operator $P: \mathcal{S}_{\omega}^{\prime}\left(\mathbb{R}^{d}\right) \rightarrow \mathcal{S}_{\omega}^{\prime}\left(\mathbb{R}^{d}\right)$ is $\omega$-regular if given $u \in \mathcal{S}_{\omega}^{\prime}\left(\mathbb{R}^{d}\right)$ such that $P u \in \mathcal{S}_{\omega}\left(\mathbb{R}^{d}\right)$, then we have $u \in \mathcal{S}_{\omega}\left(\mathbb{R}^{d}\right)$. See [13] for a study of $\omega$-regularity of linear partial differential operators with polynomial coefficients using quadratic transformations (cf. [53] for the non-isotropic case).

In this section, we provide a sufficient condition for global $\omega$-regularity of a pseudodifferential operator. We use the method of the parametrix. The proof is based on [50, 65]. The result will follow the lines of [2, 32] (cf. [25]). The following estimate is proved in [11, Proposition 2.1].

Lemma 3.1. Let $\omega$ be a subadditive weight function. For all $\lambda>0$ and $j, k \in \mathbb{N}_{0}$, we have

$$
\frac{e^{\lambda \varphi_{\omega}^{*}\left(\frac{j}{\lambda}\right)}}{j !} \frac{e^{\lambda \varphi_{\omega}^{*}\left(\frac{k}{\lambda}\right)}}{k !} \leq \frac{e^{\lambda \varphi_{\omega}^{*}\left(\frac{j+k}{\lambda}\right)}}{(j+k) !} .
$$

The following result is obvious and the proof is elementary (see Section 2.2).

Lemma 3.2. If $\sum a_{j} \in \mathrm{FGS}_{\rho}^{m_{1}, \omega}$ and $b(x, \xi) \in \mathrm{GS}_{\rho}^{m_{2}, \omega}$, then $\sum a_{j}(x, \xi) b(x, \xi)$ is a formal sum in $\mathrm{FGS}_{\rho}^{m_{1}+m_{2}, \omega}$.

Theorem 3.3. Let $\omega$ be a weight function and let $\sigma$ be a subadditive weight function with $\omega\left(t^{1 / \rho}\right)=o(\sigma(t))$ as $t \rightarrow \infty$. Let $p(x, \xi) \in \mathrm{GS}_{\rho}^{|m|, \omega}$ be such that, for some $R \geq 1$ :

(i) There exists $c>0$ such that $|p(x, \xi)| \geq c e^{-|m| \omega(x, \xi)}$ for $\langle(x, \xi)\rangle \geq R$;

(ii) There exist $C>0$ and $n \in \mathbb{N}$ such that

$$
\left|D_{x}^{\alpha} D_{\xi}^{\beta} p(x, \xi)\right| \leq C^{|\alpha+\beta|}\langle(x, \xi)\rangle^{-\rho|\alpha+\beta|} e^{\frac{1}{n} \varphi_{\sigma}^{*}(n|\alpha|)} e^{\frac{1}{n} \varphi_{\sigma}^{*}(n|\beta|)}|p(x, \xi)|,
$$


for $\alpha, \beta \in \mathbb{N}_{0}^{d},\langle(x, \xi)\rangle \geq R$.

Then, there exists $q(x, \xi) \in \mathrm{GS}_{\rho}^{|m|, \omega}$ such that $q \circ p \sim 1$ in $\mathrm{FGS}_{\rho}^{|m|, \omega}$.

Proof. We set

$$
q_{0}(x, \xi):=\frac{1}{p(x, \xi)}, \quad\langle(x, \xi)\rangle \geq R .
$$

We show by induction on $|\alpha+\beta| \in \mathbb{N}_{0}$ that there exists $C_{1}>0$ such that

$$
\left|D_{x}^{\alpha} D_{\xi}^{\beta} q_{0}(x, \xi)\right| \leq C_{1}^{|\alpha+\beta|}\langle(x, \xi)\rangle^{-\rho|\alpha+\beta|} e^{\frac{1}{n} \varphi_{\sigma}^{*}(n|\alpha|)} e^{\frac{1}{n} \varphi_{\sigma}^{*}(n|\beta|)}\left|q_{0}(x, \xi)\right|,
$$

for all $\alpha, \beta \in \mathbb{N}_{0}^{d},\langle(x, \xi)\rangle \geq R$. Indeed, the inequality is true for $\alpha=\beta=0$. By induction we assume the inequality $(3.1)$ holds for all $(\widetilde{\alpha}, \widetilde{\beta})<(\alpha, \beta)$. Since $p(x, \xi) q_{0}(x, \xi)=1$, we have

$$
\begin{aligned}
& p(x, \xi) D_{x}^{\alpha} D_{\xi}^{\beta} q_{0}(x, \xi) \\
& =-\sum_{0 \neq(\widetilde{\alpha}, \widetilde{\beta}) \leq(\alpha, \beta)} \frac{\alpha !}{\widetilde{\alpha} !(\alpha-\widetilde{\alpha}) !} \frac{\beta !}{\widetilde{\beta} !(\beta-\widetilde{\beta}) !} D_{x}^{\widetilde{\alpha}} D_{\xi}^{\widetilde{\beta}} p(x, \xi) D_{x}^{\alpha-\widetilde{\alpha}} D_{\xi}^{\beta-\widetilde{\beta}} q_{0}(x, \xi) .
\end{aligned}
$$

Therefore, by condition (ii) and by the inductive hypothesis, we obtain

$$
\begin{aligned}
\left|p(x, \xi) D_{x}^{\alpha} D_{\xi}^{\beta} q_{0}(x, \xi)\right| \leq & \sum_{0 \neq(\widetilde{\alpha}, \widetilde{\beta}) \leq(\alpha, \beta)} \frac{\alpha !}{\widetilde{\alpha} !(\alpha-\widetilde{\alpha}) !} \frac{\beta !}{\widetilde{\beta} !(\beta-\widetilde{\beta}) !} C^{|\widetilde{\alpha}+\widetilde{\beta}|}\langle(x, \xi)\rangle^{-\rho|\widetilde{\alpha}+\widetilde{\beta}|} \times \\
& \times e^{\frac{1}{n} \varphi_{\sigma}^{*}(n|\widetilde{\alpha}|)} e^{\frac{1}{n} \varphi_{\sigma}^{*}(n|\widetilde{\beta}|)}|p(x, \xi)| C_{1}^{|\alpha-\widetilde{\alpha}+\beta-\widetilde{\beta}|} \times \\
& \times\langle(x, \xi)\rangle^{-\rho|\alpha-\widetilde{\alpha}+\beta-\widetilde{\beta}|} e^{\frac{1}{n} \varphi_{\sigma}^{*}(n|\alpha-\widetilde{\alpha}|)} e^{\frac{1}{n} \varphi_{\sigma}^{*}(n|\beta-\widetilde{\beta}|)}\left|q_{0}(x, \xi)\right| .
\end{aligned}
$$

As $\frac{\alpha !}{\widetilde{\alpha} !(\alpha-\widetilde{\alpha}) !} \widetilde{\beta !(\beta-\widetilde{\beta}) !} \leq \frac{|\alpha| !}{|\widetilde{\alpha}| ! \alpha-\widetilde{\alpha} \mid !} \frac{|\beta| ! \mid}{|\widetilde{\beta}| !|\beta-\widetilde{\beta}| !}($ Lemma 0.1), we get, by Lemma 3.1,

$$
|\alpha| ! \frac{e^{\frac{1}{n} \varphi_{\sigma}^{*}(n|\widetilde{\alpha}|)}}{|\widetilde{\alpha}| !} \frac{e^{\frac{1}{n} \varphi_{\sigma}^{*}(n|\alpha-\widetilde{\alpha}|)}}{|\alpha-\widetilde{\alpha}| !}|\beta| ! \frac{e^{\frac{1}{n} \varphi_{\sigma}^{*}(n|\widetilde{\beta}|)}}{|\widetilde{\beta}| !} \frac{e^{\frac{1}{n} \varphi_{\sigma}^{*}(n|\beta-\widetilde{\beta}|)}}{|\beta-\widetilde{\beta}| !} \leq e^{\frac{1}{n} \varphi_{\sigma}^{*}(n|\alpha|)} e^{\frac{1}{n} \varphi_{\sigma}^{*}(n|\beta|)} .
$$

Thus,

$$
\begin{aligned}
& \left|D_{x}^{\alpha} D_{\xi}^{\beta} q_{0}(x, \xi)\right| \\
& \leq C_{1}^{|\alpha+\beta|}\langle(x, \xi)\rangle^{-\rho|\alpha+\beta|} e^{\frac{1}{n} \varphi_{\sigma}^{*}(n|\alpha|)} e^{\frac{1}{n} \varphi_{\sigma}^{*}(n|\beta|)}\left|q_{0}(x, \xi)\right| \sum_{0 \neq(\widetilde{\alpha}, \widetilde{\beta}) \leq(\alpha, \beta)}\left(\frac{C}{C_{1}}\right)^{|\widetilde{\alpha}+\widetilde{\beta}|} .
\end{aligned}
$$


We take $C_{1}>0$ large enough so that

$$
\sum_{k=1}^{\infty}\left(\frac{d C}{C_{1}}\right)^{k}<1
$$

With this, we obtain, by Lemma 0.1(1),

$$
\sum_{0 \neq(\widetilde{\alpha}, \widetilde{\beta}) \leq(\alpha, \beta)}\left(\frac{C}{C_{1}}\right)^{|\widetilde{\alpha}+\widetilde{\beta}|} \leq \sum_{k=1}^{|\alpha+\beta|} \sum_{|\eta|=k}\left(\frac{C}{C_{1}}\right)^{k} \leq \sum_{k=1}^{|\alpha+\beta|}\left(\frac{d C}{C_{1}}\right)^{k}<1,
$$

which completes the proof of (3.1). Without losing generality, assume that $C<C_{1}$.

We define recursively, for $j \in \mathbb{N}$,

$$
q_{j}(x, \xi):=-q_{0}(x, \xi) \sum_{0<|\gamma| \leq j} \frac{1}{\gamma !}\left(\partial_{\xi}^{\gamma} q_{j-|\gamma|}(x, \xi)\right)\left(D_{x}^{\gamma} p(x, \xi)\right) .
$$

We claim that there exist constants $C_{2}, C_{3}>0$ with $C_{1}<C_{2}<C_{3}$ such that

$$
\left|D_{x}^{\alpha} D_{\xi}^{\beta} q_{j}(x, \xi)\right| \leq C_{2}^{|\alpha+\beta|} C_{3}^{j}\langle(x, \xi)\rangle^{-\rho(|\alpha+\beta|+2 j)} e^{\frac{1}{n} \varphi_{\sigma}^{*}(n(|\alpha+\beta|+2 j))} e^{|m| \omega(x, \xi)},
$$

for all $\alpha, \beta \in \mathbb{N}_{0}^{d},\langle(x, \xi)\rangle \geq R$. We proceed by induction on $j \in \mathbb{N}_{0}$. If $j=0$, then formula (3.1) implies formula (3.2) since $\left|q_{0}(x, \xi)\right| \leq(1 / c) e^{|m| \omega(x, \xi)}$ for $\langle(x, \xi)\rangle \geq R$ (by condition $(i))$. Now, fix $j \in \mathbb{N}$. By induction, we assume that (3.2) holds for all $0 \leq \ell<j$ for some constants $C_{2}, C_{3}>0$ large enough satisfying $C_{3}>C_{2}>C_{1}$ to be determined. The derivatives of $q_{j}(x, \xi)$ are estimated by

$$
\begin{aligned}
\left|D_{x}^{\alpha} D_{\xi}^{\beta} q_{j}(x, \xi)\right| \leq & \sum_{\substack{\alpha_{1}+\alpha_{2}+\alpha_{3}=\alpha \\
\beta_{1}+\beta_{2}+\beta_{3}=\beta}} \frac{\alpha !}{\alpha_{1} ! \alpha_{2} ! \alpha_{3} !} \frac{\beta !}{\beta_{1} ! \beta_{2} ! \beta_{3} !}\left|D_{x}^{\alpha_{1}} D_{\xi}^{\beta_{1}} q_{0}(x, \xi)\right| \times \\
& \times \sum_{0<|\gamma| \leq j} \frac{1}{\gamma !}\left|D_{x}^{\alpha_{2}} D_{\xi}^{\beta_{2}+\gamma} q_{j-|\gamma|}(x, \xi)\right|\left|D_{x}^{\alpha_{3}+\gamma} D_{\xi}^{\beta_{3}} p(x, \xi)\right| .
\end{aligned}
$$


By (3.1), (3.2), and condition (ii), we have

$$
\begin{aligned}
\mid D_{x}^{\alpha} & D_{\xi}^{\beta} q_{j}(x, \xi) \mid \\
\leq & \sum_{\substack{\alpha_{1}+\alpha_{2}+\alpha_{3}=\alpha \\
\beta_{1}+\beta_{2}+\beta_{3}=\beta}} \frac{\alpha !}{\alpha_{1} ! \alpha_{2} ! \alpha_{3} !} \frac{\beta !}{\beta_{1} ! \beta_{2} ! \beta_{3} !} C_{1}^{\left|\alpha_{1}+\beta_{1}\right|}\langle(x, \xi)\rangle^{-\rho\left|\alpha_{1}+\beta_{1}\right|} e^{\frac{1}{n} \varphi_{\sigma}^{*}\left(n\left|\alpha_{1}\right|\right)} \times \\
& \times e^{\frac{1}{n} \varphi_{\sigma}^{*}\left(n\left|\beta_{1}\right|\right)}\left|q_{0}(x, \xi)\right| \sum_{0<|\gamma| \leq j} \frac{1}{\gamma !} C_{2}^{\left|\alpha_{2}+\beta_{2}+\gamma\right|} C_{3}^{j-|\gamma|} \times \\
& \times\langle(x, \xi)\rangle^{-\rho\left(\left|\alpha_{2}+\beta_{2}+\gamma\right|+2(j-|\gamma|)\right)} e^{\frac{1}{n} \varphi_{\sigma}^{*}\left(n\left(\left|\alpha_{2}+\beta_{2}+\gamma\right|+2(j-|\gamma|)\right)\right)} e^{|m| \omega(x, \xi)} \times \\
& \times C^{\left|\alpha_{3}+\gamma+\beta_{3}\right|}\langle(x, \xi)\rangle^{-\rho\left|\alpha_{3}+\gamma+\beta_{3}\right|} e^{\frac{1}{n} \varphi_{\sigma}^{*}\left(n\left|\alpha_{3}+\gamma\right|\right)} e^{\frac{1}{n} \varphi_{\sigma}^{*}\left(n\left|\beta_{3}\right|\right)}|p(x, \xi)| \\
= & \langle(x, \xi)\rangle^{-\rho(|\alpha+\beta|+2 j)} e^{|m| \omega(x, \xi)} \sum_{\beta_{1}+\beta_{2}+\beta_{3}=\beta} \frac{\alpha !}{\alpha_{1} ! \alpha_{2} ! \alpha_{3} !} \frac{\beta !}{\beta_{1} ! \beta_{2} ! \beta_{3} !} C_{1}^{\left|\alpha_{1}+\beta_{1}\right|} \times \\
& \times e^{\frac{1}{n} \varphi_{\sigma}^{*}\left(n\left|\alpha_{1}\right|\right)} e^{\frac{1}{n} \varphi_{\sigma}^{*}\left(n\left|\beta_{1}\right|\right)} \sum_{0<|\gamma| \leq j} \frac{1}{\gamma !} C_{2}^{\left|\alpha_{2}+\beta_{2}+\gamma\right|} C_{3}^{j-|\gamma|} \times \\
& \times e^{\frac{1}{n} \varphi_{\sigma}^{*}\left(n\left(\left|\alpha_{2}+\beta_{2}\right|+2 j-|\gamma|\right)\right)} C^{\left|\alpha_{3}+\gamma+\beta_{3}\right|} e^{\frac{1}{n} \varphi_{\sigma}^{*}\left(n\left|\alpha_{3}+\gamma\right|\right)} e^{\frac{1}{n} \varphi_{\sigma}^{*}\left(n\left|\beta_{3}\right|\right)} .
\end{aligned}
$$

We multiply and divide on the right-hand side of (3.3) by

$$
\left(\left|\alpha_{2}+\beta_{2}\right|+2 j-|\gamma|\right) !\left|\alpha_{3}+\gamma\right| !\left|\beta_{3}\right| !
$$

Then, as

$$
\frac{\alpha !}{\alpha_{1} ! \alpha_{2} ! \alpha_{3} !} \frac{\beta !}{\beta_{1} ! \beta_{2} ! \beta_{3} !} \leq \frac{|\alpha| !}{\left|\alpha_{1}\right| !\left|\alpha_{2}\right| !\left|\alpha_{3}\right| !} \frac{|\beta| !}{\left|\beta_{1}\right| !\left|\beta_{2}\right| !\left|\beta_{3}\right| !},
$$

we have, by Lemma 3.1,

$$
\begin{aligned}
& \frac{e^{\frac{1}{n} \varphi_{\sigma}^{*}\left(n\left|\alpha_{1}\right|\right)}}{\left|\alpha_{1}\right| !} \frac{e^{\frac{1}{n} \varphi_{\sigma}^{*}\left(n\left|\beta_{1}\right|\right)}}{\left|\beta_{1}\right| !} \frac{e^{\frac{1}{n} \varphi_{\sigma}^{*}\left(n\left(\left|\alpha_{2}+\beta_{2}\right|+2 j-|\gamma|\right)\right)}}{\left(\left|\alpha_{2}+\beta_{2}\right|+2 j-|\gamma|\right) !} \frac{e^{\frac{1}{n} \varphi_{\sigma}^{*}\left(n\left|\alpha_{3}+\gamma\right|\right)}}{\left|\alpha_{3}+\gamma\right| !} \frac{e^{\frac{1}{n} \varphi_{\sigma}^{*}\left(n\left|\beta_{3}\right|\right)}}{\left|\beta_{3}\right| !} \\
& \quad \leq \frac{1}{(|\alpha+\beta|+2 j) !} e^{\frac{1}{n} \varphi_{\sigma}^{*}(n(|\alpha+\beta|+2 j))} .
\end{aligned}
$$

We check that

$$
\frac{|\alpha| !}{\left|\alpha_{2}\right| !\left|\alpha_{3}\right| !} \frac{|\beta| !}{\left|\beta_{2}\right| !\left|\beta_{3}\right| !}\left|\alpha_{3}+\gamma\right| !\left|\beta_{3}\right| ! \frac{\left(\left|\alpha_{2}+\beta_{2}\right|+2 j-|\gamma|\right) !}{(|\alpha+\beta|+2 j) !} \leq 2^{\left|\alpha_{1}+\alpha_{3}\right|} 2^{\left|\beta_{1}+\beta_{3}\right|} \text {. }
$$


Indeed, we multiply and divide by $\left(\left|\alpha_{1}+\alpha_{3}\right|+\left|\beta_{1}+\beta_{3}\right|+|\gamma|\right)$ ! on the left-hand side of (3.4), which is therefore estimated by

$$
\begin{aligned}
& \frac{|\alpha| !}{\left|\alpha_{2}\right| !\left|\alpha_{3}\right| !} \frac{|\beta| !}{\left|\beta_{2}\right| !\left|\beta_{3}\right| !} \frac{\left|\alpha_{3}+\gamma\right| !\left|\beta_{3}\right| !}{\left(\left|\alpha_{1}+\alpha_{3}\right|+\left|\beta_{1}+\beta_{3}\right|+|\gamma|\right) !} \frac{1}{\left(\begin{array}{c}
|\alpha+\beta|+2 j \\
\left|\alpha_{2}+\beta_{2}\right|+2 j-|\gamma|
\end{array}\right)} \\
& \quad \leq \frac{|\alpha| !}{\left|\alpha_{2}\right| !\left|\alpha_{3}\right| !} \frac{|\beta| !}{\left|\beta_{2}\right| !\left|\beta_{3}\right| !} \frac{1}{\left|\alpha_{1}\right| !\left|\beta_{1}\right| !} \frac{1}{\left(\begin{array}{c}
|\alpha+\beta|+2 j \\
\left|\alpha_{2}+\beta_{2}\right|+2 j-|\gamma|
\end{array}\right)} .
\end{aligned}
$$

As we have, for $\alpha=\alpha_{1}+\alpha_{2}+\alpha_{3}$,

$$
\frac{|\alpha| !}{\left|\alpha_{1}\right| !\left|\alpha_{2}\right| !\left|\alpha_{3}\right| !}=\frac{\left|\alpha_{1}+\alpha_{3}\right| !}{\left|\alpha_{1}\right| !\left|\alpha_{3}\right| !}\left(\begin{array}{c}
|\alpha| \\
\left|\alpha_{2}\right|
\end{array}\right) \leq 2^{\left|\alpha_{1}+\alpha_{3}\right|}\left(\begin{array}{c}
|\alpha| \\
\left|\alpha_{2}\right|
\end{array}\right),
$$

(and similarly for $\beta=\beta_{1}+\beta_{2}+\beta_{3}$ ), we deduce formula (3.4) by an application of Lemma 0.1(5):

$$
\left(\begin{array}{c}
|\alpha| \\
\left|\alpha_{2}\right|
\end{array}\right)\left(\begin{array}{c}
|\beta| \\
\left|\beta_{2}\right|
\end{array}\right) \leq\left(\begin{array}{c}
|\alpha+\beta| \\
\left|\alpha_{2}+\beta_{2}\right|
\end{array}\right) \leq\left(\begin{array}{c}
|\alpha+\beta|+2 j \\
\left|\alpha_{2}+\beta_{2}\right|+2 j-|\gamma|
\end{array}\right)
$$

We then have from (3.3),

$$
\begin{aligned}
\left|D_{x}^{\alpha} D_{\xi}^{\beta} q_{j}(x, \xi)\right| \leq & \langle(x, \xi)\rangle^{-\rho(|\alpha+\beta|+2 j)} e^{\frac{1}{n} \varphi_{\sigma}^{*}(n(|\alpha+\beta|+2 j))} e^{|m| \omega(x, \xi)} \times \\
& \times \sum_{\substack{\alpha_{1}+\alpha_{2}+\alpha_{3}=\alpha \\
\beta_{1}+\beta_{2}+\beta_{3}=\beta}} 2^{\left|\alpha_{1}+\alpha_{3}\right|} 2^{\left|\beta_{1}+\beta_{3}\right|} C_{1}^{\left|\alpha_{1}+\beta_{1}\right|} C_{2}^{\left|\alpha_{2}+\beta_{2}\right|} \times \\
& \times C_{3}^{j} C^{\left|\alpha_{3}+\beta_{3}\right|} \sum_{0<|\gamma| \leq j} \frac{1}{\gamma !} C_{2}^{|\gamma|} C_{3}^{-|\gamma|} C^{|\gamma|} .
\end{aligned}
$$

Since $C<C_{1}$, we have

$$
\begin{aligned}
& C_{2}^{|\alpha+\beta|} C_{3}^{j} \sum_{\substack{\alpha \\
\alpha_{1}+\alpha_{2}+\alpha_{3}=\alpha \\
\beta_{1}+\beta_{3}=\beta}}\left(\frac{2 C_{1}}{C_{2}}\right)^{\left|\alpha_{1}+\beta_{1}\right|}\left(\frac{2 C}{C_{2}}\right)^{\left|\alpha_{3}+\beta_{3}\right|} \\
& \leq C_{2}^{|\alpha+\beta|} C_{3}^{j} \sum_{\substack{\alpha_{1}+\alpha_{2}+\alpha_{3}=\alpha \\
\beta_{1}+\beta_{2}+\beta_{3}=\beta}}\left(\frac{2 C_{1}}{C_{2}}\right)^{\left|\alpha_{1}+\alpha_{3}+\beta_{1}+\beta_{3}\right|} \leq C_{2}^{|\alpha+\beta|} C_{3}^{j} \sum_{k=0}^{|\alpha+\beta|} \sum_{|\eta|=k}\left(\frac{2 C_{1}}{C_{2}}\right)^{k} .
\end{aligned}
$$

Thus, we take, according to Lemma 0.1(1), $C_{2}>0$ large enough so that

$$
\sum_{k=0}^{\infty}\left(\frac{2 d C_{1}}{C_{2}}\right)^{k}<2
$$


Now, the remaining sum is estimated, using Lemma 0.1, by

$$
\sum_{0<|\gamma| \leq j} \frac{1}{\gamma !}\left(\frac{C C_{2}}{C_{3}}\right)^{|\gamma|} \leq \sum_{k=1}^{j} \frac{d^{k}}{k !}\left(\frac{C C_{2}}{C_{3}}\right)^{k} \sum_{|\gamma|=k} 1 \leq \sum_{k=1}^{j} \frac{\left(d^{2}\right)^{k}}{k !}\left(\frac{C C_{2}}{C_{3}}\right)^{k} .
$$

Hence, taking $C_{3}>0$ large enough so that

$$
\sum_{k=1}^{\infty} \frac{1}{k !}\left(\frac{d^{2} C C_{2}}{C_{3}}\right)^{k}<1 / 2
$$

we prove (3.2). By Lemma $0.10(1)$, for all $\lambda>0$ there exists $C_{\lambda}>0$ such that for each $j \in \mathbb{N}_{0}$,

$$
\left|D_{x}^{\alpha} D_{\xi}^{\beta} q_{j}(x, \xi)\right| \leq C_{\lambda} C_{2}^{|\alpha+\beta|} C_{3}^{j}\langle(x, \xi)\rangle^{-\rho(|\alpha+\beta|+2 j)} e^{\lambda \rho \varphi_{\omega}^{*}\left(\frac{|\alpha+\beta|+2 j}{\lambda}\right)} e^{|m| \omega(x, \xi)}
$$

for all $\alpha, \beta \in \mathbb{N}_{0}^{d},\langle(x, \xi)\rangle \geq R$, and the estimate (2.1) in Definition 2.1 hence follows.

We extend $q_{j}$ to the whole $\mathbb{R}^{2 d}$ for all $j \in \mathbb{N}_{0}$ in the following way: we take $\phi \in \mathcal{D}_{(\sigma)}\left(\mathbb{R}^{2 d}\right)$, supported in $\left\{(x, \xi) \in \mathbb{R}^{2 d}:\langle(x, \xi)\rangle \leq 2 R\right\}$ and $\phi \equiv 1$ in $\left\{(x, \xi) \in \mathbb{R}^{2 d}:\langle(x, \xi)\rangle \leq R\right\}$. Hence for all $j \in \mathbb{N}_{0}, \widetilde{q}_{j}:=q_{j}(1-\phi) \in C^{\infty}\left(\mathbb{R}^{2 d}\right)$ satisfies that $\widetilde{q}_{j}=q_{j}$ in $\langle(x, \xi)\rangle>2 R$ and vanishes if $\langle(x, \xi)\rangle \leq R$. Since $1-\phi \in \mathrm{GS}_{\rho}^{0, \omega}$, by Lemma 3.2, we have $\sum \widetilde{q}_{j} \in \mathrm{FGS}_{\rho}^{|m|, \omega}$. By abuse of notation we write $\sum q_{j}$ for the formal sum $\sum \widetilde{q_{j}}$.

We show $\sum q_{j} \circ p \sim 1$. We denote $\sum r_{j}=\sum q_{j} \circ p$ as in Proposition 2.15 for $\tau=0$. In fact, if $j \in \mathbb{N}$, we have

$$
\begin{aligned}
q_{j}(x, \xi) p(x, \xi) & =-\sum_{0<|\gamma| \leq j} \frac{1}{\gamma !}\left(\partial_{\xi}^{\gamma} q_{j-|\gamma|}(x, \xi)\right)\left(D_{x}^{\gamma} p(x, \xi)\right) \\
& =-r_{j}(x, \xi)+q_{j}(x, \xi) p(x, \xi)
\end{aligned}
$$

Hence $r_{j}(x, \xi)=0$ for all $j \in \mathbb{N}$. Moreover, $r_{0}(x, \xi)=q_{0}(x, \xi) p(x, \xi)=1$ if $\langle(x, \xi)\rangle \geq 2 R$. Therefore $\sum q_{j} \circ p \sim 1$. Finally, by Theorem 2.6 there exists $q \in \mathrm{GS}_{\rho}^{|m|, \omega}$ such that $q \sim \sum q_{j}$. So, by Proposition 2.17 we have $q \circ p \sim 1$ as we wanted.

Corollary 3.4. Let $\omega$ and $\sigma$ be as in Theorem 3.3. If p satisfies the hypotheses in Theorem 3.3, then its corresponding pseudodifferential operator $P$ is $\omega$ regular. 
Proof. By Theorem 3.3, there exists a pseudodifferential operator $Q$ such that $Q \circ P=I+R$, where $I$ is the identity operator, and $R$ is an $\omega$-regularizing operator. Then, for every $u \in \mathcal{S}_{\omega}^{\prime}\left(\mathbb{R}^{d}\right)$ we have

$$
u=Q(P u)-R u \text {. }
$$

Therefore, if $P u \in \mathcal{S}_{\omega}\left(\mathbb{R}^{d}\right)$, we have $u \in \mathcal{S}_{\omega}\left(\mathbb{R}^{d}\right)$.

By Theorem 2.24, we observe that if $P$ is $\omega$-regular, then $P_{\tau}$ is $\omega$-regular, for all $\tau \in \mathbb{R}$.

Given two global symbols $a$ and $b$, we write $a \# b$ for the Weyl product of $a$ and $b$, that is, the symbol corresponding to the composition of the Weyl quantizations of $a$ and $b$ :

$$
(a \# b)^{w}(x, D)=a^{w}(x, D) b^{w}(x, D) .
$$

We observe that the Weyl product of $a$ and $b$ has the following asymptotic expansion (cf. Corollary 2.32):

$$
(a \# b)(x, \xi) \sim \sum_{j=0}^{\infty} \sum_{|\beta+\gamma|=j} \frac{(-1)^{|\beta|}}{\gamma ! \beta !} 2^{-|\beta+\gamma|} \partial_{\xi}^{\gamma} D_{x}^{\beta} a(x, \xi) \partial_{\xi}^{\beta} D_{x}^{\gamma} b(x, \xi) .
$$

Let $p \in \mathrm{GS}_{\rho}^{|m|, \omega}$ be as in Theorem 3.3. Then, there exists $q \in \mathrm{GS}_{\rho}^{|m|, \omega}$ such that the associated pseudodifferential operators $P$ and $Q$ satisfy $Q \circ P=I+R$, where $I$ is the identity operator, and $R$ is an $\omega$-regularizing operator. Hence, by Theorem 2.24, we can write the pseudodifferential operator associated to the amplitude $p\left(\frac{x+y}{2}, \xi\right), P^{w}(x, D)$, by $P+R^{\prime}$, for some $\omega$-regularizing operator $R^{\prime}$, and also for $q\left(\frac{x+y}{2}, \xi\right)$, namely $Q^{w}(x, D)=Q+R^{\prime \prime}$, for some $\omega$-regularizing operator $R^{\prime \prime}$. Thus, using Proposition 1.18,

$$
\begin{aligned}
Q^{w}(x, D) \circ P^{w}(x, D) & =\left(Q+R^{\prime \prime}\right) \circ\left(P+R^{\prime}\right) \\
& =Q \circ P+R^{\prime \prime} \circ P+Q \circ R^{\prime}+R^{\prime \prime} \circ R^{\prime}=I+R^{\prime \prime \prime},
\end{aligned}
$$

for some $\omega$-regularizing operator $R^{\prime \prime \prime}$. Therefore, we have

Corollary 3.5. Let $p \in \mathrm{GS}_{\rho}^{|m|, \omega}$ satisfy the hypotheses in Theorem 3.3. Then, there exists $q \in \mathrm{GS}_{\rho}^{|m|, \omega}$ such that $q \# p \sim 1$. 


\subsection{Example}

Now, we construct a global symbol $p(x, \xi)$ with prescribed exponential growth in all the variables satisfying the conditions in Theorem 3.3 for Gevrey weights $\omega(t)=t^{a}, 0<a<1$. It is inspired by [3, Capitolo 4].

We start by considering

$$
f(t)=e^{t^{b}}, \quad 0 \leq b<1 / 2, \quad t \geq 1 .
$$

First, we show that, for all $n \in \mathbb{N}$,

$$
\begin{aligned}
f^{(n)}(t) & =\left(a_{0, n} t^{n b-n}+a_{1, n} t^{(n-1) b-n}+a_{2, n} t^{(n-2) b-n}+\cdots+a_{n-1, n} t^{b-n}\right) e^{t^{b}} \\
& =\frac{a_{0, n}+a_{1, n} t^{-b}+a_{2, n} t^{-2 b}+\cdots+a_{n-1, n} t^{-(n-1) b}}{t^{n(1-b)}} e^{t^{b}},
\end{aligned}
$$

where

$$
\begin{aligned}
a_{0, n} & =b^{n}, \quad n \geq 1 ; \\
a_{k, n} & =a_{k, n-1} b+a_{k-1, n-1}((n-k) b-(n-1)), \quad 1 \leq k \leq n-2, n \geq 3 ; \\
a_{n-1, n} & =b(b-1)(b-2) \cdots(b-n+1), \quad n \geq 2,
\end{aligned}
$$

and

$$
\left|a_{k, n}\right| \leq(b+1)^{n} n^{k}, \quad 0 \leq k \leq n-1 .
$$

It is clear that if $b=0$, then $f(t)=e$. Therefore, its derivatives are always zero, hence we consider $b>0$. We proceed by induction on $n \in \mathbb{N}$. If $n=1$, then $f^{\prime}(t)=b t^{b-1} e^{t^{b}}=a_{0,1} t^{b-1} e^{t^{b}}$, and the result is clearly true. If we assume that $f$ fulfils the statement for $n \in \mathbb{N}$, then we have that $f^{(n+1)}(t)=\left(f^{(n)}\right)^{\prime}(t)$ is equal to

$$
\begin{aligned}
& \left(a_{0, n} t^{n b-n}+a_{1, n} t^{(n-1) b-n}+a_{2, n} t^{(n-2) b-n}+\cdots+a_{n-1, n} t^{b-n}\right) e^{t^{b}} b t^{b-1}+ \\
& \quad+\left(a_{0, n}(n b-n) t^{n b-(n+1)}+a_{1, n}((n-1) b-n) t^{(n-1) b-(n+1)}+\right. \\
& \left.\quad+a_{2, n}((n-2) b-n) t^{(n-2) b-(n+1)}+\cdots+a_{n-1, n}(b-n) t^{b-(n+1)}\right) e^{t^{b}} \\
& =\left(a_{0, n} b t^{(n+1) b-(n+1)}+\left(a_{1, n} b+a_{0, n}(n b-n)\right) t^{n b-(n+1)}+\right. \\
& \left.\quad+\left(a_{2, n} b+a_{1, n}((n-1) b-n)\right) t^{(n-1) b-(n+1)}+\cdots+a_{n-1, n}(b-n) t^{b-(n+1)}\right) e^{t^{b}} .
\end{aligned}
$$


We write

$$
\begin{aligned}
& a_{0, n+1}:=a_{0, n} b=b^{n} b=b^{n+1}, \\
& a_{k, n+1}:=a_{k, n} b+a_{k-1, n}((n+1-k) b-n), \quad 1 \leq k \leq n-1, \\
& a_{n, n+1}:=a_{n-1, n}(b-n)=b(b-1) \cdots(b-n+1)(b-n) .
\end{aligned}
$$

Now, we estimate $\left|a_{k, n+1}\right|, 0 \leq k \leq n$. If $k=0$, formula (3.7) is true by definition of $a_{0, n+1}$. For $1 \leq k \leq n-1$, since $b<1 / 2$, we have

$$
\left|\frac{n+1-k}{n} b-1\right| \leq 1 \text {. }
$$

Then, using the induction hypothesis, we have

$$
\begin{aligned}
\left|a_{k, n+1}\right| & \leq\left|a_{k, n}\right| b+\left|a_{k-1, n}\right||(n+1-k) b-n| \\
& \leq(b+1)^{n} n^{k} b+(b+1)^{n} n^{k-1}|(n+1-k) b-n| \\
& =(b+1)^{n} n^{k}\left(b+\left|\frac{n+1-k}{n} b-1\right|\right) \leq(b+1)^{n+1}(n+1)^{k} .
\end{aligned}
$$

Finally, if $k=n$, as $0<b<1 / 2$, we obtain

$$
\begin{aligned}
\left|a_{n, n+1}\right| & =|b||b-1||b-2| \cdots|b-n+1| \\
& =b(1-b)(2-b) \cdots(n-1-b) \leq b \cdot 1 \cdot 2 \cdots(n-1)=(n-1) ! b .
\end{aligned}
$$

It is straightforward to check by induction that, for all $n \in \mathbb{N}$,

$$
(n-1) ! b \leq(b+1)^{n} n^{n-1} .
$$

Indeed, if $n=1$, the formula yields $b \leq b+1$. If $n>1$, then

$$
n ! b=n(n-1) ! b \leq(b+1)^{n} n^{n} \leq(b+1)^{n+1}(n+1)^{n} .
$$

This shows the estimates in (3.7).

Now, let

$$
g(t)=e^{m t^{b}}, \quad 0 \leq b<1 / 2, \quad m \in \mathbb{R}, \quad t \geq 1 .
$$

For all $n \in \mathbb{N}$, we have that $g^{(n)}(t)$ is equal to

$$
\frac{a_{0, n} m^{n}+a_{1, n} m^{n-1} t^{-b}+a_{2, n} m^{n-2} t^{-2 b}+\cdots+a_{n-1, n} m t^{-(n-1) b}}{t^{n(1-b)}} e^{m t^{b}} .
$$

We also show

$$
\left|g^{(n)}(t)\right| \leq(1+|m|)^{n} \frac{((b+1) e)^{n} n !}{t^{n(1-b)}} e^{|m| t^{b}} .
$$


Indeed, for $b=0$, the function $g(t)$ is reduced to a constant, and this case is excluded from the general case. Moreover, as $g(t)=f\left(m^{1 / b} t\right)$, formula (3.9) is satisfied. To check (3.10), from the estimates of the coefficients of the derivatives for $f$, we have, for $t \geq 1$,

$$
\begin{aligned}
\left|g^{(n)}(t)\right| \leq & \frac{\left|a_{0, n}\right|(1+|m|)^{n}+\left|a_{1, n}\right|(1+|m|)^{n-1}+\left|a_{2, n}\right|(1+|m|)^{n-2}}{t^{n(1-b)}}+\cdots+ \\
& \left.\quad+\frac{\left|a_{n-1, n}\right|(1+|m|)}{t^{n(1-b)}}\right) e^{m t^{b}} \\
\leq & (1+|m|)^{n} \frac{\left|a_{0, n}\right|+\left|a_{1, n}\right|+\left|a_{2, n}\right|+\cdots+\left|a_{n-1, n}\right|}{t^{n(1-b)}} e^{m t^{b}} \\
\leq & (1+|m|)^{n} \frac{(b+1)^{n}\left(1+n+n^{2}+\cdots+n^{n-1}\right)}{t^{n(1-b)}} e^{m t^{b}} \\
\leq & (1+|m|)^{n} \frac{(b+1)^{n} n^{n}}{t^{n(1-b)}} e^{m t^{b}} .
\end{aligned}
$$

Since $n^{n} \leq e^{n} n$ ! for every $n \in \mathbb{N}$ we obtain (3.10) for all $n \in \mathbb{N}, t \geq 1$ and $m \in \mathbb{R}$.

Now, writing the function $g$ in (3.8) as

$$
g(t)=e^{m t^{a / 2}}, \quad 0 \leq a<1, \quad m \in \mathbb{R}, \quad t \geq 1,
$$

and setting

$$
u(z)=\langle z\rangle^{2}=1+|z|^{2}, \quad z \in \mathbb{R}^{2 d},
$$

we define

$$
p(z):=g(u(z))=e^{m\langle z\rangle^{a}}, \quad 0 \leq a<1, \quad m \in \mathbb{R}, \quad z \in \mathbb{R}^{2 d} .
$$

We investigate the behaviour of its derivatives. We again omit the case $a=0$, because it is trivial.

We use Faà di Bruno formula for several variables (see for example [52, Page 234]):

$$
D^{\alpha} p(z)=\sum_{0 \leq k \leq|\alpha|} g^{(k)}(u(z)) \alpha ! \sum_{*} \prod_{|\beta|>0} \frac{1}{c_{\beta} !}\left(\frac{D^{\beta} u(z)}{\beta !}\right)^{c_{\beta}},
$$

for all $\alpha \in \mathbb{N}_{0}^{2 d}$ and $z \in \mathbb{R}^{2 d}$, where the sum $\sum_{*}$ runs over all $c_{\beta} \in \mathbb{N}_{0}$ such that $\sum_{|\beta|>0} c_{\beta}=k$ and $\sum_{|\beta|>0} \beta c_{\beta}=\alpha$. The derivatives of $u$ are always zero, except when $\beta=e_{j}$ or $\beta=2 e_{j}$, where $e_{j} \in \mathbb{N}_{0}^{2 d}$ is the canonical basis for $1 \leq j \leq 2 d$. For these cases, we have

$$
D^{e_{j}} u(z)=2 z_{j} ; \quad D^{2 e_{j}} u(z)=2, \quad z=\left(z_{1}, \ldots, z_{2 d}\right) \in \mathbb{R}^{2 d}, \quad j=1, \ldots, 2 d .
$$


Therefore, we obtain

$$
\prod_{|\beta|>0} \frac{1}{c_{\beta} !}\left|\frac{D^{\beta} u(z)}{\beta !}\right|^{c_{\beta}}=\prod_{j=1}^{2 d} \frac{1}{c_{e_{j}} !} \frac{1}{c_{2 e_{j}} !}\left|2 z_{j}\right|^{c_{e_{j}}} .
$$

Moreover, we have

$$
\begin{aligned}
k & =\sum_{|\beta|>0} c_{\beta}=\sum_{j=1}^{2 d}\left(c_{e_{j}}+c_{2 e_{j}}\right) \in \mathbb{N}_{0} \\
\alpha & =\left(\alpha_{1}, \ldots, \alpha_{2 d}\right)=\sum_{|\beta|>0} \beta c_{\beta}=\left(c_{e_{1}}+2 c_{2 e_{1}}, \ldots, c_{e_{2 d}}+2 c_{2 e_{2 d}}\right) \in \mathbb{N}_{0}^{2 d} .
\end{aligned}
$$

Thus, $\sum_{j=1}^{2 d} c_{e_{j}}=2 k-|\alpha| \leq|\alpha|$, and therefore

$$
\prod_{j=1}^{2 d}\left|2 z_{j}\right|^{c_{e_{j}}} \leq(2\langle z\rangle)^{2 k-|\alpha|} \leq 2^{|\alpha|}\langle z\rangle^{2 k-|\alpha|}
$$

Then, by (3.10), we obtain, from (3.12),

$$
\begin{aligned}
\left|D^{\alpha} p(z)\right| \leq & 2^{|\alpha|} \sum_{0 \leq k \leq|\alpha|}(1+|m|)^{k} \frac{((a / 2+1) e)^{k} k !}{\langle z\rangle^{2 k(1-a / 2)}} e^{m\langle z\rangle^{a}} \\
& \times \alpha !\langle z\rangle^{2 k-|\alpha|} \sum_{*} \prod_{j=1}^{2 d} \frac{1}{c_{e_{j}} !} \frac{1}{c_{2 e_{j}} !} \\
\leq & 2^{|\alpha|}(1+|m|)^{|\alpha|}((a / 2+1) e)^{|\alpha|} \alpha !\langle z\rangle^{-(1-a)|\alpha|} e^{m\langle z\rangle^{a} \times} \\
& \times \sum_{0 \leq k \leq|\alpha|} k ! \sum_{*} \prod_{j=1}^{2 d} \frac{1}{c_{e_{j}} !} \frac{1}{c_{2 e_{j}} !}
\end{aligned}
$$

Finally we have, by Lemma 0.1(4),

$$
\begin{aligned}
\sum_{*} k ! \prod_{j=1}^{2 d} \frac{1}{c_{e_{j}} !} \frac{1}{c_{2 e_{j}} !}= & \sum_{\substack{c_{e_{1}}, \ldots c_{e_{2 d}}, c_{2 e_{1}}, \ldots, c_{2 e_{2 d}} \in \mathbb{N}_{0}: \\
\sum_{l=1}^{2 d}\left(c_{e_{l}}+c_{2 e_{l}}\right)=k, c_{e_{j}}+2 c_{2 e_{j}}=\alpha_{j}, 1 \leq j \leq 2 d}} \frac{k !}{c_{e_{1}} ! \cdots c_{e_{2 d}} ! c_{2 e_{1}} ! \cdots c_{2 e_{2 d}} !} \\
\leq & \sum_{\substack{c_{j} \in \mathbb{N}_{0}: \\
\sum_{l=1}^{2 d}\left(c_{e_{l}}+c_{2 e_{l}}\right)=k}} \frac{k !}{c_{e_{1}} ! \cdots c_{e_{2 d}} ! c_{2 e_{1}} ! \cdots c_{2 e_{2 d}} !}=(4 d)^{k} \leq(4 d)^{|\alpha|}
\end{aligned}
$$


Since $\sum_{0 \leq k \leq|\alpha|} 1 \leq|\alpha|+1 \leq 2^{|\alpha|}$, we obtain that there exists

$$
C=16 d(1+|m|)(a / 2+1) e>0
$$

such that for all $\alpha \in \mathbb{N}_{0}^{2 d}$ and $z \in \mathbb{R}^{2 d}$,

$$
\left|D^{\alpha} p(z)\right| \leq C^{|\alpha|} \alpha !\langle z\rangle^{-(1-a)|\alpha|}|p(z)| .
$$

Now, we want to show that $p$, similarly as in (3.11), that is,

$$
p(x, \xi):=e^{|m|\langle(x, \xi)\rangle^{a}}, \quad 0<a<1, x, \xi \in \mathbb{R}^{d},
$$

is a global symbol in $\mathrm{GS}_{\rho}^{|m|, \omega}$ for $\omega(t)=t^{a}$ and $\rho:=1-a$, and it satisfies the sufficient conditions imposed in Theorem 3.3. Indeed, it is trivial to check that condition $(i)$ of Theorem 3.3 holds. On the other hand, by (3.15), we have

$$
\left|D_{x}^{\alpha} D_{\xi}^{\beta} p(x, \xi)\right| \leq C^{|\alpha+\beta|} \alpha ! \beta !\langle(x, \xi)\rangle^{-\rho|\alpha+\beta|} e^{|m| \omega(x, \xi)}, \quad \alpha, \beta \in \mathbb{N}_{0}^{d}, x, \xi \in \mathbb{R}^{d} .
$$

Take $\sigma$ as in Theorem 3.3, and then use (0.12) to get that, for some $D>0$,

$$
\alpha ! \beta ! \leq D e^{\varphi_{\sigma}^{*}(|\alpha|)} e^{\varphi_{\sigma}^{*}(|\beta|)} .
$$

This shows that condition ( $i i)$ is verified. To show that $p$ is a global symbol in $\mathrm{GS}_{\rho}^{|m|, \omega}$ it is enough to use Lemma 0.9.

We observe that to use Lemma 0.9 , we need to assume that, as $a=1-\rho$, $\omega\left(t^{1 / \rho}\right)=t^{(1-\rho) / \rho}=o(t)$ as $t \rightarrow \infty$. So,

$$
\frac{1-\rho}{\rho}<1
$$

Hence, $1 / 2<\rho \leq 1$.

\subsection{Global hypoellipticity}

Definition 3.6. We say that $p \in \mathrm{GS}_{\rho}^{m, \omega}$ is an $\omega$-hypoelliptic symbol in the class $\mathrm{HGS}_{\rho}^{m, m_{0} ; \omega}$ if there exist $R \geq 1$ and a Gevrey weight function $\sigma$ (i.e. $\sigma(t)=t^{a}$ for some $\left.0<a<1\right)$ satisfying $\omega\left(t^{1 / \rho}\right)=o(\sigma(t))$ as $t \rightarrow \infty$ such that

(i) There exist $c>0$ such that $c e^{m_{0} \omega(x, \xi)} \leq|p(x, \xi)|$ for $\langle(x, \xi)\rangle \geq R$. 
(ii) There exist $C>0, n \in \mathbb{N}$ such that

$$
\begin{aligned}
& \quad\left|D_{x}^{\alpha} D_{\xi}^{\beta} p(x, \xi)\right| \leq C^{|\alpha+\beta|}\langle(x, \xi)\rangle^{-\rho|\alpha+\beta|} e^{\frac{1}{n} \varphi_{\sigma}^{*}(n|\alpha|)} e^{\frac{1}{n} \varphi_{\sigma}^{*}(n|\beta|)}|p(x, \xi)|, \\
& \text { for }\langle(x, \xi)\rangle \geq R, \quad \alpha, \beta \in \mathbb{N}_{0}^{d} .
\end{aligned}
$$

It follows that if $p \in \operatorname{HGS}_{\rho}^{m, m_{0} ; \omega}$, then there exist $c, C>0$ such that

$$
c e^{m_{0} \omega(x, \xi)} \leq|p(x, \xi)| \leq C e^{m \omega(x, \xi)}, \quad \text { for }|(x, \xi)| \text { large enough. }
$$

Therefore, for such $p$, with $m_{0} \geq-|m|$, we obtain the thesis in Theorem 3.3. Then, any pseudodifferential operator defined by an $\omega$-hypoelliptic symbol as in Definition 3.6 (with $m_{0} \geq-|m|$ ) is also $\omega$-regular in the sense of Theorem 3.3. On the other hand, the twisted Laplacian in $\mathbb{R}^{2}$

$$
L=\left(D_{x}-\frac{1}{2} y\right)^{2}+\left(D_{y}-\frac{1}{2} x\right)^{2}
$$

is an example of an $\omega$-regular operator for every weight function $\omega[13$, Example 5.4], but not $\omega$-hypoelliptic [13, Remark 5.5] since the symbol $(\xi-y / 2)^{2}+$ $(\eta+x / 2)^{2}$ of $L$ fails to satisfy condition $(i)$ of Definition 3.6, as it vanishes for $\xi=y / 2, \eta=-x / 2$.

In Theorem 3.14 below we show that Definition 3.6 is independent on the quantization $\tau$ for the case $m_{0}=m$. Hence, we extend [9, Proposition 8.4] showing that $\omega$-hypoelliptic symbol classes are invariant by a change of quantization.

In Example 2.12, we have seen that the formal sum considered may change the order $m$ given by the amplitude $a \in \mathrm{GA}_{\rho}^{m, \omega}$. For this reason, here we develop a symbolic calculus for global mixed classes very similar to the one in Section 2.1, which keeps this order. The symbols are defined as:

Definition 3.7. We say that $p \in \widetilde{\mathrm{GS}}_{\rho}^{m, \omega}$ if $p \in C^{\infty}\left(\mathbb{R}^{2 d}\right)$ and there exists a Gevrey weight function $\sigma$ satisfying $\omega\left(t^{1 / \rho}\right)=o(\sigma(t))$ as $t \rightarrow \infty$ such that for all $\lambda>0$ there is $C_{\lambda}>0$ with

$$
\left|D_{x}^{\alpha} D_{\xi}^{\beta} p(x, \xi)\right| \leq C_{\lambda}\langle(x, \xi)\rangle^{-\rho|\alpha+\beta|} e^{\lambda \varphi_{\sigma}^{*}\left(\frac{|\alpha+\beta|}{\lambda}\right)} e^{m \omega(x, \xi)}, \quad \alpha, \beta \in \mathbb{N}_{0}^{d}, x, \xi \in \mathbb{R}^{d} .
$$

We remark that Definitions 3.6 and 3.7 are independent of the weight $\sigma$, since if $\sigma_{1}$ and $\sigma_{2}$ are Gevrey weight functions satisfying $\omega\left(t^{1 / \rho}\right)=o\left(\sigma_{j}(t)\right)$ as $t \rightarrow \infty$, $j=1,2$, the Gevrey weight function

$$
\sigma(t):=\max \left\{\sigma_{1}(t), \sigma_{2}(t)\right\}, \quad \text { for } t>1
$$


(hence $\left.\varphi_{\sigma}^{*} \geq \max \left\{\varphi_{\sigma_{1}}^{*}, \varphi_{\sigma_{2}}^{*}\right\}\right)$ satisfies $\omega\left(t^{1 / \rho}\right)=o(\sigma(t))$ as $t \rightarrow \infty$. On the other hand, by Lemma $0.10(1)$ it follows that $\widetilde{\mathrm{GS}}_{\rho}^{m, \omega} \subseteq \mathrm{GS}_{\rho}^{m, \omega}$.

Lemma 3.8. Let $p \in{\widetilde{\mathrm{GS}_{\rho}}}^{m, \omega}$. We have $p \in \mathrm{HGS}_{\rho}^{m, m ; \omega}$ if and only if there exist $R \geq 1$ and $c>0$ such that $|p(x, \xi)| \geq c e^{m \omega(x, \xi)}$ for $\langle(x, \xi)\rangle \geq R$.

Proof. It is enough to see that if $p$ satisfies that estimate from below, then $p \in \operatorname{HGS}_{\rho}^{m, m ; \omega}$. Since $p \in \widetilde{\mathrm{GS}}_{\rho}^{m, \omega}$ there exists $C>0$ such that, for some Gevrey weight $\sigma$ with $\omega\left(t^{1 / \rho}\right)=o(\sigma(t))$ as $t \rightarrow \infty$,

$$
\left|D_{x}^{\alpha} D_{\xi}^{\beta} p(x, \xi)\right| \leq C\langle(x, \xi)\rangle^{-\rho|\alpha+\beta|} e^{\varphi_{\sigma}^{*}(|\alpha+\beta|)} e^{m \omega(x, \xi)},
$$

for all $\alpha, \beta \in \mathbb{N}_{0}^{d}$ and $x, \xi \in \mathbb{R}^{d}$, which in particular yields

$$
c e^{m \omega(x, \xi)} \leq|p(x, \xi)| \leq C e^{m \omega(x, \xi)}, \quad\langle(x, \xi)\rangle \geq R .
$$

This shows condition $(i)$ of Definition 3.6. To see that condition $(i i)$ of Definition 3.6 holds, we have from (3.17), using (3.18) and (0.11),

$$
\left|D_{x}^{\alpha} D_{\xi}^{\beta} p(x, \xi)\right| \leq \frac{C}{c}\langle(x, \xi)\rangle^{-\rho|\alpha+\beta|} e^{\frac{1}{2} \varphi_{\sigma}^{*}(2|\alpha|)} e^{\frac{1}{2} \varphi_{\sigma}^{*}(2|\beta|)}|p(x, \xi)|,
$$

for all $\alpha, \beta \in \mathbb{N}_{0}^{d},\langle(x, \xi)\rangle \geq R$. Since $p \in \widetilde{\mathrm{GS}}_{\rho}^{m, \omega} \subseteq \mathrm{GS}_{\rho}^{m, \omega}$ we get $p \in \operatorname{HGS}_{\rho}^{m, m ; \omega}$.

For the corresponding definitions of amplitude and formal sums, we consider similar mixed conditions.

Definition 3.9. An amplitude $a(x, y, \xi) \in C^{\infty}\left(\mathbb{R}^{3 d}\right)$ belongs to $\widetilde{\mathrm{GA}}_{\rho}^{m, \omega}$ if there exists a Gevrey weight function $\sigma$ satisfying $\omega\left(t^{1 / \rho}\right)=o(\sigma(t))$ as $t \rightarrow \infty$ such that for all $\lambda>0$ there is $C_{\lambda}>0$ with

$$
\left|D_{x}^{\alpha} D_{y}^{\gamma} D_{\xi}^{\beta} a(x, y, \xi)\right| \leq C_{\lambda}\left(\frac{\langle x-y\rangle}{\langle(x, y, \xi)\rangle}\right)^{\rho|\alpha+\gamma+\beta|} e^{\lambda \varphi_{\sigma}^{*}\left(\frac{|\alpha+\gamma+\beta|}{\lambda}\right)} e^{m \omega(x, \xi)},
$$

for all $\alpha, \gamma, \beta \in \mathbb{N}_{0}^{d}$, and $x, y, \xi \in \mathbb{R}^{d}$.

Definition 3.10. A formal sum $\sum a_{j}$ is in $\widetilde{\mathrm{FGS}}_{\rho}^{m, \omega}$ if $a_{j} \in C^{\infty}\left(\mathbb{R}^{2 d}\right)$ and there exist $R \geq 1$ and a Gevrey weight function $\sigma$ satisfying $\omega\left(t^{1 / \rho}\right)=o(\sigma(t))$ as $t \rightarrow \infty$ such that for all $n \in \mathbb{N}$ there exists $C_{n}>0$ such that

$$
\left|D_{x}^{\alpha} D_{\xi}^{\beta} a_{j}(x, \xi)\right| \leq C_{n}\langle(x, \xi)\rangle^{-\rho(|\alpha+\beta|+j)} e^{n \varphi_{\sigma}^{*}\left(\frac{|\alpha+\beta|+j}{n}\right)} e^{m \omega(x, \xi)},
$$

for each $j \in \mathbb{N}_{0}, \alpha, \beta \in \mathbb{N}_{0}^{d}, \log \left(\frac{\langle(x, \xi)\rangle}{R}\right) \geq \frac{n}{j} \varphi_{\omega}^{*}\left(\frac{j}{n}\right)$. 
Definition 3.11. We say that $\sum a_{j}$ and $\sum b_{j}$ in $\widetilde{\mathrm{FGS}}_{\rho}^{m, \omega}$ are equivalent, denoted by $\sum a_{j} \sim \sum b_{j}$, if there exist $R \geq 1$ and a Gevrey weight function $\sigma$ satisfying $\omega\left(t^{1 / \rho}\right)=o(\sigma(t))$ as $t \rightarrow \infty$ such that for all $n \in \mathbb{N}$ there are $C_{n}>0$ and $N_{n} \in \mathbb{N}$ such that

$$
\left|D_{x}^{\alpha} D_{\xi}^{\beta} \sum_{j<N}\left(a_{j}-b_{j}\right)\right| \leq C_{n}\langle(x, \xi)\rangle^{-\rho(|\alpha+\beta|+N)} e^{n \varphi_{\sigma}^{*}\left(\frac{|\alpha+\beta|+N}{n}\right)} e^{m \omega(x, \xi)},
$$

for all $N \geq N_{n}, \alpha, \beta \in \mathbb{N}_{0}^{d}, \log \left(\frac{\langle(x, \xi)\rangle}{R}\right) \geq \frac{n}{N} \varphi_{\omega}^{*}\left(\frac{N}{n}\right)$.

Again by Lemma $0.10(1)$, we have that $\widetilde{\mathrm{GA}}_{\rho}^{m, \omega} \subseteq \mathrm{GA}_{\rho}^{m, \omega}$ if $m \geq 0$, and $\widetilde{\mathrm{FGS}}_{\rho}^{m, \omega} \subseteq \mathrm{FGS}_{\rho}^{m, \omega}$ for $m \in \mathbb{R}$. These new definitions permit to keep the same order $m \geq 0$ for some results in Chapter 2. For instance, if $a \in{\widetilde{\mathrm{GA}_{\rho}}}^{m, \omega}$, then the formal sum in Example 2.12 belongs to $\widetilde{\mathrm{FGS}}_{\rho}^{m, \omega}, m \geq 0$.

Furthermore, it can be shown that the function $\Psi_{j}$ defined in (2.15) (see also (2.4)) belongs to $\widetilde{\mathrm{GS}}_{\rho}^{0, \omega}$. Hence, all the symbolic calculus studied in Section 2.1 can be reproduced in the same way, with the difference that we now preserve the order $m$. In fact, we have

Theorem 3.12. Let $a(x, y, \xi)$ be an amplitude in $\widetilde{\mathrm{GA}}_{\rho}^{m, \omega}$ with $m \geq 0$, and let $A$ be its associated pseudodifferential operator $A$. Then, for any $\tau \in \mathbb{R}$, we can write $A$ as

$$
A=P+R,
$$

where $R$ is an $\omega$-regularizing operator and $P$ is the operator given by

$$
P u(x)=\iint e^{i(x-y) \cdot \xi} p((1-\tau) x+\tau y, \xi) u(y) d y d \xi, \quad u \in \mathcal{S}_{\omega}\left(\mathbb{R}^{d}\right),
$$

being $p \in{\widetilde{\mathrm{GS}_{\rho}}}^{m, \omega}$. Moreover,

$$
\left.p(x, \xi) \sim \sum_{j=0}^{\infty} \sum_{|\beta+\gamma|=j} \frac{1}{\beta ! \gamma !} \tau^{|\beta|}(1-\tau)^{|\gamma|} \partial_{\xi}^{\beta+\gamma}\left(-D_{x}\right)^{\beta} D_{y}^{\gamma} a(x, y, \xi)\right|_{y=x} .
$$

In this case, we can also proceed as in [64] (see after Theorem 2.24), to obtain $p_{\tau}$ such that its associated pseudodifferential operator $P_{\tau}$ is equal to $A$. It is called the $\tau$-symbol of the pseudodifferential operator associated to the amplitude $a \in{\widetilde{\mathrm{GA}_{\rho}}}_{\rho}^{m}$. We also obtain the relation between two given $\tau$-symbols (cf. Theorem 2.27). 
Theorem 3.13. If $a_{\tau_{1}}, a_{\tau_{2}} \in \widetilde{\mathrm{GS}}_{\rho}^{m, \omega}$ are the $\tau_{1}$-symbol and the $\tau_{2}$-symbol of $a$ pseudodifferential operator $A$, then, in $\widetilde{\mathrm{FGS}}_{\rho}^{m, \omega}$,

$$
a_{\tau_{2}}(x, \xi) \sim \sum_{j=0}^{\infty} \sum_{|\alpha|=j} \frac{1}{\alpha !}\left(\tau_{1}-\tau_{2}\right)^{|\alpha|} \partial_{\xi}^{\alpha} D_{x}^{\alpha} a_{\tau_{1}}(x, \xi) .
$$

Now, we show that if a global symbol with mixed conditions satisfies (3.16) for some quantization, then so any quantization.

Theorem 3.14. Let $a_{\tau_{1}} \in \widetilde{\mathrm{GS}}_{\rho}^{m, \omega}$ for some $\tau_{1} \in \mathbb{R}$. If $a_{\tau_{1}} \in \mathrm{HGS}_{\rho}^{m, m ; \omega}$, then $a_{\tau_{2}} \in \mathrm{HGS}_{\rho}^{m, m ; \omega}$ for all $\tau_{2} \in \mathbb{R}$.

Proof. By Theorem 3.12 we have that $a_{\tau_{2}} \in \widetilde{\mathrm{GS}}_{\rho}^{m, \omega}$. Then, by Lemma 3.8, it is enough to find $R \geq 1$ and $c>0$ such that

$$
\left|a_{\tau_{2}}(x, \xi)\right| \geq c e^{m \omega(x, \xi)}, \quad \text { for }\langle(x, \xi)\rangle \geq R .
$$

From Lemma 3.8, we obtain by assumption that there are $R_{1} \geq 1$ and $D_{1}>0$ such that

$$
\left|a_{\tau_{1}}(x, \xi)\right| \geq D_{1} e^{m \omega(x, \xi)}, \quad \text { for }\langle(x, \xi)\rangle \geq R_{1} .
$$

From Theorem 3.13 we have (see Definition 3.11; by simplicity we assume $N_{n}=1, n \in \mathbb{N}$ ) that there exist $R_{2} \geq 1$ and a Gevrey weight function $\sigma_{1}$ satisfying $\omega\left(t^{1 / \rho}\right)=o\left(\sigma_{1}(t)\right)$ for $t \rightarrow \infty$ such that for some $C_{1}>0, N_{1} \in \mathbb{N}$,

$$
\left|a_{\tau_{2}}(x, \xi)-\sum_{j<N} \sum_{|\alpha|=j} \frac{1}{\alpha !}\left(\tau_{1}-\tau_{2}\right)^{|\alpha|} \partial_{\xi}^{\alpha} D_{x}^{\alpha} a_{\tau_{1}}(x, \xi)\right| \leq C_{1}\langle(x, \xi)\rangle^{-\rho N} e^{\varphi_{\sigma_{1}}^{*}(N)} e^{m \omega(x, \xi)},
$$

for all $N \geq N_{1}$ and $\log \left(\frac{\langle(x, \xi)\rangle}{R_{2}}\right) \geq \frac{1}{N} \varphi_{\omega}^{*}(N)$. From Lemma 0.10(1), there exists $A_{1}>0$ so that $\varphi_{\sigma_{1}}^{*}(N) \leq A_{1}+\rho \varphi_{\omega}^{*}(N)$ for all $N \in \mathbb{N}$. Therefore, for some $R_{3} \geq R_{2}$ determined later,

$$
\left|a_{\tau_{2}}(x, \xi)-\sum_{j<N} \sum_{|\alpha|=j} \frac{1}{\alpha !}\left(\tau_{1}-\tau_{2}\right)^{|\alpha|} \partial_{\xi}^{\alpha} D_{x}^{\alpha} a_{\tau_{1}}(x, \xi)\right| \leq C_{1} e^{A_{1}} R_{3}^{-\rho N} e^{m \omega(x, \xi)},
$$

for all $N \geq N_{1}$ and $\log \left(\frac{\langle(x, \xi)\rangle}{R_{3}}\right) \geq \frac{1}{N} \varphi_{\omega}^{*}(N)$. 
Now, we fix $N:=N_{1} \in \mathbb{N}$. We have

$$
\begin{aligned}
\left|a_{\tau_{2}}(x, \xi)\right| \geq & \left|\sum_{j=0}^{N-1} \sum_{|\alpha|=j} \frac{1}{\alpha !}\left(\tau_{1}-\tau_{2}\right)^{|\alpha|} \partial_{\xi}^{\alpha} D_{x}^{\alpha} a_{\tau_{1}}(x, \xi)\right|- \\
& -\left|a_{\tau_{2}}(x, \xi)-\sum_{j<N} \sum_{|\alpha|=j} \frac{1}{\alpha !}\left(\tau_{1}-\tau_{2}\right)^{|\alpha|} \partial_{\xi}^{\alpha} D_{x}^{\alpha} a_{\tau_{1}}(x, \xi)\right| .
\end{aligned}
$$

We show that

$$
\left|\sum_{j=0}^{N-1} \sum_{|\alpha|=j} \frac{1}{\alpha !}\left(\tau_{1}-\tau_{2}\right)^{|\alpha|} \partial_{\xi}^{\alpha} D_{x}^{\alpha} a_{\tau_{1}}(x, \xi)\right| \geq \frac{D_{1}}{2} e^{m \omega(x, \xi)},
$$

for $\langle(x, \xi)\rangle$ large enough. For $N=1$, formula (3.22) holds by (3.20) for $\langle(x, \xi)\rangle \geq R_{1}$. Hence, we assume $N>1$. We first estimate

$$
\left|\sum_{j=1}^{N-1} \sum_{|\alpha|=j} \frac{1}{\alpha !}\left(\tau_{1}-\tau_{2}\right)^{|\alpha|} \partial_{\xi}^{\alpha} D_{x}^{\alpha} a_{\tau_{1}}(x, \xi)\right| .
$$

As $a_{\tau_{1}}(x, \xi) \in \widetilde{\mathrm{GS}}_{\rho}^{m, \omega}$, there exists a Gevrey weight function $\sigma_{2}$ satisfying $\omega\left(t^{1 / \rho}\right)=o\left(\sigma_{2}(t)\right)$ for $t \rightarrow \infty$ such that, for some $C_{2}>0$ we have

$$
\begin{aligned}
\left|D_{x}^{\alpha} D_{\xi}^{\alpha} a_{\tau_{1}}(x, \xi)\right| & \leq C_{2}\langle(x, \xi)\rangle^{-\rho(2|\alpha|)} e^{\varphi_{\sigma_{2}}^{*}(2|\alpha|)} e^{m \omega(x, \xi)} \\
& \leq C_{2}\langle(x, \xi)\rangle^{-\rho} e^{\varphi_{\sigma_{2}}^{*}(2(N-1))} e^{m \omega(x, \xi)}
\end{aligned}
$$

for all $x, \xi \in \mathbb{R}^{d}$ and $1 \leq|\alpha| \leq N-1$. Again by Lemma $0.10(1)$, there exists $A_{2}>0$ such that $\varphi_{\sigma_{2}}^{*}(2(N-1)) \leq A_{2}+\rho \varphi_{\omega}^{*}(2(N-1))$. For $R_{4} \geq 1$ to be determined, satisfying

$$
\log \left(\frac{\langle(x, \xi)\rangle}{R_{4}}\right) \geq \varphi_{\omega}^{*}(2(N-1))
$$

we have

$$
\begin{aligned}
\left|D_{x}^{\alpha} D_{\xi}^{\alpha} a_{\tau_{1}}(x, \xi)\right| & \leq C_{2} e^{A_{2}}\langle(x, \xi)\rangle^{-\rho} e^{\rho \varphi_{\omega}^{*}(2(N-1))} e^{m \omega(x, \xi)} \\
& \leq C_{2} e^{A_{2}}\left(R_{4}\right)^{-\rho} e^{m \omega(x, \xi)}
\end{aligned}
$$

for all $\langle(x, \xi)\rangle \geq R_{4} e^{\varphi_{\omega}^{*}(2(N-1))}$ and all $1 \leq|\alpha| \leq N-1$. On the other hand, by Lemma 0.1 we get

$$
\sum_{j=1}^{N-1} \sum_{|\alpha|=j} \frac{\left|\tau_{1}-\tau_{2}\right|^{|\alpha|}}{\alpha !} \leq \sum_{j=1}^{N-1} \frac{\left(d\left|\tau_{1}-\tau_{2}\right|\right)^{j}}{j !} \sum_{|\alpha|=j} 1 \leq e^{d^{2}\left|\tau_{1}-\tau_{2}\right|}
$$


and then we obtain

$$
\left|\sum_{j=1}^{N-1} \sum_{|\alpha|=j} \frac{1}{\alpha !}\left(\tau_{1}-\tau_{2}\right)^{|\alpha|} \partial_{\xi}^{\alpha} D_{x}^{\alpha} a_{\tau_{1}}(x, \xi)\right| \leq C_{2} e^{A_{2}}\left(R_{4}\right)^{-\rho} e^{d^{2}\left|\tau_{1}-\tau_{2}\right|} e^{m \omega(x, \xi)},
$$

for all $\langle(x, \xi)\rangle \geq R_{4} e^{\varphi_{\omega}^{*}(2(N-1))}$. Hence, we take $R_{4} \geq 1$ such that

$$
\left(R_{4}\right)^{\rho} \geq \frac{2}{D_{1}} C_{2} e^{A_{2}} e^{d^{2}\left|\tau_{1}-\tau_{2}\right|}
$$

From formulas (3.23) and (3.20) we then obtain

$$
\begin{aligned}
& \left|\sum_{j=0}^{N-1} \sum_{|\alpha|=j} \frac{1}{\alpha !}\left(\tau_{1}-\tau_{2}\right)^{|\alpha|} \partial_{\xi}^{\alpha} D_{x}^{\alpha} a_{\tau_{1}}(x, \xi)\right| \\
& \quad \geq D_{1} e^{m \omega(x, \xi)}-C_{2} e^{A_{2}}\left(R_{4}\right)^{-\rho} e^{d^{2}\left|\tau_{1}-\tau_{2}\right|} e^{m \omega(x, \xi)} \geq \frac{D_{1}}{2} e^{m \omega(x, \xi)},
\end{aligned}
$$

and we show $(3.22)$ for $\langle(x, \xi)\rangle \geq \max \left\{R_{1}, R_{4} e^{\varphi_{\omega}^{*}(2(N-1))}\right\}$. Finally, put $R_{3} \geq 1$ such that

$$
R_{3}^{\rho N} \geq \frac{4}{D_{1}} C_{1} e^{A_{1}}
$$

Thus, for

$$
R:=\max \left\{R_{1}, R_{4} e^{\varphi_{\omega}^{*}(2(N-1))}, R_{3} e^{\frac{1}{N} \varphi_{\omega}^{*}(N)}\right\}
$$

we obtain by (3.22) and (3.21)

$$
\left|a_{\tau_{2}}(x, \xi)\right| \geq \frac{D_{1}}{2} e^{m \omega(x, \xi)}-C_{1} e^{A_{1}} R_{3}^{-\rho N} e^{m \omega(x, \xi)} \geq \frac{D_{1}}{4} e^{m \omega(x, \xi)},
$$

for $\langle(x, \xi)\rangle \geq R$. Hence, we get, for $c:=D_{1} / 4>0$, the inequality in (3.19) and the proof is complete. 



\section{Chapter 4}

\section{The Weyl wave front set}

In the theory of partial differential equations, the wave front set locates the singularities of a distribution and, at the same time, describes the directions of the high frequencies (in terms of the Fourier transform) responsible for those singularities. In the classical context of Schwartz distributions theory, it was originally defined by Hörmander [44]. There is a lot of literature on wave front sets for the study of the regularity of linear partial differential operators in spaces of distributions or ultradistributions in a local sense; see for instance $[1,2,10,12,34,60,61]$ and the references therein.

In global classes of functions and distributions (like the Schwartz class $\mathcal{S}\left(\mathbb{R}^{d}\right)$ and its dual) the concept of singular support does not make sense, since we require the information on the whole $\mathbb{R}^{d}$. However, we can still define a global wave front set to describe the micro-regularity of a distribution. In fact, Hörmander [45] introduced two different types of global wave front set addressed to the study of quadratic hyperbolic operators: the $C^{\infty}$ wave front set, in the Beurling setting, for temperate distributions $u \in \mathcal{S}^{\prime}\left(\mathbb{R}^{d}\right)$ using Weyl quantizations, and the analytic wave front set, in the Roumieu setting, for ultradistributions of Gelfand-Shilov type. Unfortunately, these global versions of wave front set have been almost ignored in the literature. Very recently, Rodino and Wahlberg [61] recover the concept of $C^{\infty}$ wave front set of [45] and show that it can be reformulated in terms of the short-time Fourier transform. Moreover, in [61] the authors also show that the original wave front set 
coincides with the Beurling version of the analytic wave front set introduced by Hörmander. On the other hand, Nakamura [54] introduced the homogeneous wave front set for the study of propagation of micro-singularities for Schrödinger equations, and it turns out to be equal to the wave front set [63]. Cappiello and Schulz [26] recovered the analytic wave front set of [45] and studied some cases not treated by Hörmander for Gelfand-Shilov ultradistributions of Gevrey type.

In Boiti, Jornet, and Oliaro [14], the authors introduce the ultradifferentiable version of the analytic wave front set found in [26, 39,61] in the Beurling setting for $\mathcal{S}_{\omega}^{\prime}\left(\mathbb{R}^{d}\right)$-ultradistributions and apply it to the study of the global regularity of (pseudo)differential operators of infinite order (in [61] the authors cannot treat operators of infinite order, since they consider symbols with polynomial growth only). However, the question if the latter wave front set can also be described in terms of Weyl quantizations, as in [45, 61], remained open in the ultradifferentiable setting.

The purpose of this last chapter is twofold: on the one hand, to define the Weyl wave front set, in accordance with the conditions in Theorem 3.3, and study when it coincides with the continuous version of the wave front set defined in [14] for the ultradifferentiable setting; on the other hand, to provide some applications of this wave front set to the regularity of pseudodifferential operators of Chapter 1.

The chapter is organised as follows: In Section 4.1, we recover the definition of wave front set of [14] (Definition 4.1) defined with the short-time Fourier transform and extend the inclusion in [14, Theorem 4.13] to any differential operator with variable coefficients for this wave front set. Later, we analyse the kernel of some operators given by Weyl quantizations for symbols as in Definition 1.1 to show that the wave front set of the action of the Weyl operator on a distribution in $\mathcal{S}_{\omega}^{\prime}\left(\mathbb{R}^{d}\right)$ is in the conic support of the corresponding symbol. In Section 4.2 we introduce a new wave front set called the Weyl wave front set, and see that it can be characterized in terms of symbols of order zero. Then, we compare the wave front set given in Definition 4.1 with the Weyl wave front set. It is crucial the inclusion mentioned above about the conic support. We need also here to impose that our weight functions be smaller than some Gevrey weight (see Remark 4.20). Unfortunately, we could not circumvent this restriction, since we use similar techniques as in [61]. Finally, in Section 4.3 we study the regularity of Weyl quantizations with respect to the Weyl wave front set. For instance, for a suitable weight function $\omega$, any $0<\rho \leq 1$ and a symbol $a(x, \xi)$ as in Definition 1.1, we are able to prove that, 
see Theorem 4.31,

$$
\begin{aligned}
\mathrm{WF}_{\rho}^{\omega}\left(a^{w}(x, D) u\right) & \subset \mathrm{WF}_{\rho}^{\omega}(u) \cap \operatorname{conesupp}(a) \\
& \subset \mathrm{WF}_{\rho}^{\omega}(u) \subset \mathrm{WF}_{\rho}^{\omega}\left(a^{w}(x, D) u\right) \cup \operatorname{char}(a),
\end{aligned}
$$

for all $u \in \mathcal{S}_{\omega}^{\prime}\left(\mathbb{R}^{d}\right)$, where $a^{w}(x, D) u$ is the action of the Weyl quantization for the symbol $a$ on $u$, conesupp $(a)$ is the conic support of $a(x, \xi)$ (see Definition 4.2), $\operatorname{char}(a)$ is the characteristic set of $a(x, \xi)$ (the set of points which are characteristic for $a(x, \xi)$; see Definition 4.11), and $\mathrm{WF}_{\rho}^{\omega}(u)$ is the Weyl wave front set of $u$.

This chapter is based on the preprint [5].

\subsection{The $\omega$-wave front set}

We first introduce the global wave front set defined in [14] for ultradistributions in $\mathcal{S}_{\omega}^{\prime}\left(\mathbb{R}^{d}\right)$, given in terms of the decay of the short-time Fourier transform, in conical sets.

Definition 4.1. Let $u \in \mathcal{S}_{\omega}^{\prime}\left(\mathbb{R}^{d}\right)$ and $\psi \in \mathcal{S}_{\omega}\left(\mathbb{R}^{d}\right) \backslash\{0\}$. We say that $z_{0} \in$ $\mathbb{R}^{2 d} \backslash\{0\}$ is not in the $\omega$-wave front set $\mathrm{WF}_{\omega}^{\prime}(u)$ of $u$ if there exists an open conic set $\Gamma \subseteq \mathbb{R}^{2 d} \backslash\{0\}, z_{0} \in \Gamma$, such that

$$
\sup _{z \in \Gamma} e^{\lambda \omega(z)}\left|V_{\psi} u(z)\right|<\infty, \quad \lambda>0
$$

The wave front set $\mathrm{WF}_{\omega}^{\prime}(u)$ is a closed set in $\mathbb{R}^{2 d} \backslash\{0\}$.

We recall the following definition from [61, Definition 2.1], introduced in [45].

Definition 4.2. Let $u \in \mathcal{S}_{\omega}^{\prime}\left(\mathbb{R}^{2 d}\right)$. The conic support of $u$, conesupp $(u)$, is the set of all $z \in \mathbb{R}^{2 d} \backslash\{0\}$ such that every conic open set $\Gamma \subseteq \mathbb{R}^{2 d} \backslash\{0\}$ containing $z$ satisfies that

$$
\overline{\operatorname{supp} u \cap \Gamma} \text { is not a compact set in } \mathbb{R}^{2 d} \text {. }
$$

The conic support of $u$ is also a closed set in $\mathbb{R}^{2 d} \backslash\{0\}$.

An elementary result is

Lemma 4.3. We have

$$
\mathrm{WF}_{\omega}^{\prime}(u)=\mathrm{WF}_{\omega}^{\prime}(u+v), \quad u \in \mathcal{S}_{\omega}^{\prime}\left(\mathbb{R}^{d}\right), v \in \mathcal{S}_{\omega}\left(\mathbb{R}^{d}\right) .
$$


Proof. Fix $0 \neq \psi \in \mathcal{S}_{\omega}\left(\mathbb{R}^{d}\right)$. Let $0 \neq z_{0} \notin \mathrm{WF}_{\omega}^{\prime}(u)$. Then there exists an open conic set $\Gamma \subseteq \mathbb{R}^{2 d} \backslash\{0\}$ containing $z_{0}$ such that

$$
\sup _{z \in \Gamma} e^{\lambda \omega(z)}\left|V_{\psi} u(z)\right|<\infty, \quad \lambda>0 .
$$

Since $v \in \mathcal{S}_{\omega}\left(\mathbb{R}^{d}\right)$, by Theorem 0.28 we obtain that for all $\lambda>0$ there exists $C_{\lambda}>0$ such that

$$
e^{\lambda \omega(z)}\left|V_{\psi} v(z)\right| \leq C_{\lambda}, \quad z \in \mathbb{R}^{2 d} .
$$

Therefore, as $\left|V_{\psi}(u+v)(z)\right| \leq\left|V_{\psi} u(z)\right|+\left|V_{\psi} v(z)\right|$, we have

$$
\sup _{z \in \Gamma} e^{\lambda \omega(z)}\left|V_{\psi}(u+v)(z)\right|<\infty, \quad \lambda>0 .
$$

Hence $z_{0} \notin \mathrm{WF}_{\omega}^{\prime}(u+v)$.

For the other inclusion, we have

$$
\mathrm{WF}_{\omega}^{\prime}(u)=\mathrm{WF}_{\omega}^{\prime}(u+v-v) \subseteq \mathrm{WF}_{\omega}^{\prime}(u+v) .
$$

In [14, Theorem 4.13], the authors show that a differential operator with polynomial coefficients, namely, for some $m \in \mathbb{N}$, an operator of the form

$$
A(x, D)=\sum_{|\alpha+\beta| \leq m} c_{\alpha \beta} x^{\alpha} D_{x}^{\beta}
$$

where $c_{\alpha \beta} \in \mathbb{C}$, satisfies

$$
\mathrm{WF}_{\omega}^{\prime}(A(x, D) u) \subseteq \mathrm{WF}_{\omega}^{\prime}(u), \quad u \in \mathcal{S}_{\omega}^{\prime}\left(\mathbb{R}^{d}\right) .
$$

Here, we extend this inclusion for linear partial differential operators of the form

$$
P(x, D)=\sum_{|\gamma| \leq m} a_{\gamma}(x) D^{\gamma},
$$

for some $m \in \mathbb{N}$, where $a_{\gamma} \in \mathcal{S}_{\omega}\left(\mathbb{R}^{d}\right)$. We recall that, in general, a function in $\mathcal{S}_{\omega}\left(\mathbb{R}^{2 d}\right)$ might not be a global symbol in the class $\operatorname{GS}_{\rho}^{m, \omega}$ for some $0<\rho \leq 1$ and $m \in \mathbb{R}$. Hence, (4.1) is not necessarily an operator with symbol in $\mathrm{GS}_{\rho}^{\bar{m}, \omega}$. We have shown in Example 1.21(b) that for $\omega(t)=\log ^{s}(1+t), s \geq 1, t \geq 0$, the corresponding space $\mathcal{S}_{\omega}\left(\mathbb{R}^{2 d}\right)$ equals $\bigcap_{m \in \mathbb{R}} \mathrm{GS}_{\rho}^{m, \omega}$ (see (1.22)). 
The $\omega$-wave front set $\mathrm{WF}_{\omega}^{\prime}(u)$ is independent of the window function $\psi$. We need the following lemma, which is a refinement of [14, Proposition 3.2] for bounded sets. Throughout this chapter, let $S_{2 d-1}$ denote the unit sphere in $\mathbb{R}^{2 d}$.

Lemma 4.4. Let $u \in \mathcal{S}_{\omega}^{\prime}\left(\mathbb{R}^{d}\right), \psi \in \mathcal{S}_{\omega}\left(\mathbb{R}^{d}\right) \backslash\{0\}$, and $z_{0} \in \mathbb{R}^{2 d} \backslash\{0\}$. If there exists an open conic set $\Gamma \subseteq \mathbb{R}^{2 d} \backslash\{0\}$ containing $z_{0}$ such that

$$
\sup _{z \in \Gamma} e^{\lambda \omega(z)}\left|V_{\psi} u(z)\right|<\infty, \quad \lambda>0
$$

then, for any bounded set $\mathcal{B}$ of $\mathcal{S}_{\omega}\left(\mathbb{R}^{d}\right) \backslash\{0\}$ and for any open conic set $\Gamma^{\prime} \subseteq$ $\mathbb{R}^{2 d} \backslash\{0\}$ containing $z_{0}$ and such that $\overline{\Gamma^{\prime} \cap S_{2 d-1}} \subseteq \Gamma$, we have

$$
\sup _{\phi \in \mathcal{B}} \sup _{z \in \Gamma^{\prime}} e^{\lambda \omega(z)}\left|V_{\phi} u(z)\right|<\infty, \quad \lambda>0
$$

Proof. By Proposition 0.27, for any $\psi, \phi \in \mathcal{S}_{\omega}\left(\mathbb{R}^{d}\right) \backslash\{0\}$, we have

$$
\left|V_{\phi} u(z)\right| \leq(2 \pi)^{-d}\|\psi\|_{L^{2}\left(\mathbb{R}^{d}\right)}^{-2}\left(\left|V_{\psi} u\right| *\left|V_{\phi} \psi\right|\right)(z), \quad z \in \mathbb{R}^{2 d} .
$$

By Lemma 0.30,

$$
\left|V_{\phi} \psi\left(z^{\prime}\right)\right|=\left|\overline{V_{\psi} \phi\left(-z^{\prime}\right)}\right|=\left|V_{\psi} \phi\left(-z^{\prime}\right)\right|, \quad z^{\prime} \in \mathbb{R}^{2 d} .
$$

Then,

$$
\begin{aligned}
\left(\left|V_{\psi} u\right| *\left|V_{\phi} \psi\right|\right)(z) & =\int_{\mathbb{R}^{2 d}}\left|V_{\psi} u\left(z-z^{\prime}\right)\right|\left|V_{\phi} \psi\left(z^{\prime}\right)\right| d z^{\prime} \\
& =\int_{\mathbb{R}^{2 d}}\left|V_{\psi} u\left(z-z^{\prime}\right)\right|\left|V_{\psi} \phi\left(-z^{\prime}\right)\right| d z^{\prime}
\end{aligned}
$$

For $\varepsilon>0$, we denote for all $z \in \mathbb{R}^{2 d}$,

$$
\begin{aligned}
& I_{1}(z):=\int_{\left\langle z^{\prime}\right\rangle \leq \varepsilon\langle z\rangle}\left|V_{\psi} u\left(z-z^{\prime}\right)\right|\left|V_{\psi} \phi\left(-z^{\prime}\right)\right| d z^{\prime}, \\
& I_{2}(z):=\int_{\left\langle z^{\prime}\right\rangle>\varepsilon\langle z\rangle}\left|V_{\psi} u\left(z-z^{\prime}\right)\right|\left|V_{\psi} \phi\left(-z^{\prime}\right)\right| d z^{\prime} .
\end{aligned}
$$

We take an open conic set $\Gamma^{\prime}$ such that $z_{0} \in \Gamma^{\prime}$ and $\overline{\Gamma^{\prime} \cap S_{2 d-1}} \subseteq \Gamma$. Choose $\varepsilon>0$ sufficiently small (see, for instance, $[14,(3.25)]$ ) so that

$$
z \in \Gamma^{\prime},|z| \geq 1,\left\langle z^{\prime}\right\rangle \leq \varepsilon\langle z\rangle, \text { then } z-z^{\prime} \in \Gamma
$$


For a bounded set $\mathcal{B}$ in $\mathcal{S}_{\omega}\left(\mathbb{R}^{d}\right) \backslash\{0\}$, since $V_{\psi}: \mathcal{S}_{\omega}\left(\mathbb{R}^{d}\right) \rightarrow \mathcal{S}_{\omega}\left(\mathbb{R}^{2 d}\right)$ is continuous (by Lemma 0.26 ), the set $V_{\psi}(\mathcal{B})$ is bounded in $\mathcal{S}_{\omega}\left(\mathbb{R}^{2 d}\right)$. Thus for all $\mu>0$ there exists $C_{\mu}>0$ such that

$$
\sup _{\phi \in \mathcal{B}}\left|V_{\psi} \phi\left(-z^{\prime}\right)\right| e^{\mu \omega\left(-z^{\prime}\right)} \leq C_{\mu}, \quad z^{\prime} \in \mathbb{R}^{2 d} .
$$

To estimate $I_{1}$, we use the estimate for $\left|V_{\psi} u\right|$ in $\Gamma$ as follows: for all $\lambda>0$ there exists $C_{\lambda}>0$ such that, using (0.1),

$$
\begin{aligned}
I_{1}(z) & \leq C_{\lambda} \int_{\left\langle z^{\prime}\right\rangle \leq \varepsilon\langle z\rangle} e^{-\lambda L \omega\left(z-z^{\prime}\right)}\left|V_{\psi} \phi\left(-z^{\prime}\right)\right| d z^{\prime} \\
& \leq C_{\lambda} e^{\lambda L} e^{-\lambda \omega(z)} \int_{\mathbb{R}^{2 d}}\left|V_{\psi} \phi\left(-z^{\prime}\right)\right| e^{\lambda L \omega\left(z^{\prime}\right)} d z^{\prime} \\
& =C_{\lambda} e^{\lambda L} e^{-\lambda \omega(z)} \int_{\mathbb{R}^{2 d}}\left(\left|V_{\psi} \phi\left(-z^{\prime}\right)\right| e^{(\lambda L+1) \omega\left(-z^{\prime}\right)}\right) e^{-\omega\left(z^{\prime}\right)} d z^{\prime} \\
& \leq C_{\lambda}^{\prime} e^{-\lambda \omega(z)},
\end{aligned}
$$

for some constant $C_{\lambda}^{\prime}>0$, for all $z \in \Gamma^{\prime},|z| \geq 1$, and all $\phi \in \mathcal{B}$. Note that $\int e^{-\omega\left(z^{\prime}\right)} d z$ converges by property $(\gamma)$ of the weight $\omega$.

On the other hand, by Lemma $0.26, V_{\psi} u$ is continuous and there are constants $c, \mu>0$ such that

$$
\left|V_{\psi} u(z)\right| \leq c e^{\mu \omega(z)}, \quad z \in \mathbb{R}^{2 d} .
$$

Let $q \in \mathbb{N}_{0}$ be such that $\varepsilon^{-1}<2^{q}$. Then, for $\left\langle z^{\prime}\right\rangle>\varepsilon\langle z\rangle$, as $\omega$ is increasing, we get by condition $(\alpha)$ and $(0.6)$,

$$
\omega(z) \leq \omega\left(\varepsilon^{-1}\left\langle z^{\prime}\right\rangle\right) \leq \omega\left(2^{q}\left\langle z^{\prime}\right\rangle\right) \leq L^{q+1} \omega\left(z^{\prime}\right)+L^{q+1}+\cdots+L .
$$

Then, we have

$$
-L^{q+1} \omega\left(z^{\prime}\right) \leq-\omega(z)+\left(L^{q+1}+\cdots+L\right), \quad \text { for }\left\langle z^{\prime}\right\rangle>\varepsilon\langle z\rangle .
$$


Therefore, for all $\lambda>0$ and all $\phi \in \mathcal{B}$, we have (again by $(0.1)$ )

$$
\begin{aligned}
I_{2}(z) \leq & c \int_{\left\langle z^{\prime}\right\rangle>\varepsilon\langle z\rangle} e^{\mu \omega\left(z-z^{\prime}\right)}\left|V_{\psi} \phi\left(-z^{\prime}\right)\right| d z^{\prime} \\
\leq & c e^{\mu L} e^{\mu L \omega(z)} \int_{\left\langle z^{\prime}\right\rangle>\varepsilon\langle z\rangle} e^{\mu L \omega\left(-z^{\prime}\right)}\left|V_{\psi} \phi\left(-z^{\prime}\right)\right| d z^{\prime} \\
= & c e^{\mu L} e^{\mu L \omega(z)} \int_{\left\langle z^{\prime}\right\rangle>\varepsilon\langle z\rangle} e^{-(\lambda+\mu L) L^{q+1} \omega\left(z^{\prime}\right)} \times \\
& \times\left(\left|V_{\psi} \phi\left(-z^{\prime}\right)\right| e^{\left((\lambda+\mu L) L^{q+1}+\mu L+1\right) \omega\left(-z^{\prime}\right)}\right) e^{-\omega\left(z^{\prime}\right)} d z^{\prime} \\
\leq & c e^{\mu L} e^{(\lambda+\mu L)\left(L^{q+1}+\cdots+L\right)} e^{-\lambda \omega(z)} \times \\
& \times \int_{\mathbb{R}^{2 d}}\left(\left|V_{\psi} \phi\left(-z^{\prime}\right)\right| e^{\left((\lambda+\mu L) L^{q+1}+\mu L+1\right) \omega\left(-z^{\prime}\right)}\right) e^{-\omega\left(z^{\prime}\right)} d z^{\prime} .
\end{aligned}
$$

Hence for all $\lambda>0$ there exists $C_{\lambda}^{\prime \prime}>0$ such that

$$
I_{2}(z) \leq C_{\lambda}^{\prime \prime} e^{-\lambda \omega(z)}, \quad z \in \mathbb{R}^{2 d} .
$$

This finishes the proof.

Theorem 4.5. If $P(x, D)$ is as in (4.1), then

$$
\mathrm{WF}_{\omega}^{\prime}(P(x, D) u) \subseteq \mathrm{WF}_{\omega}^{\prime}(u), \quad u \in \mathcal{S}_{\omega}^{\prime}\left(\mathbb{R}^{d}\right)
$$

Proof. Let $0 \neq \psi \in \mathcal{S}_{\omega}\left(\mathbb{R}^{d}\right)$ be a window function. From the linearity of the short-time Fourier transform, by Lemmas 0.32 and 0.29 , we have

$$
\begin{aligned}
V_{\psi}(P(x, D) u)(x, \xi) & =\sum_{|\gamma| \leq m} V_{\psi}\left(a_{\gamma} \cdot D^{\gamma} u\right)(x, \xi) \\
& =(2 \pi)^{-d} \sum_{|\gamma| \leq m}\left(\widehat{a_{\gamma} \cdot D^{\gamma}} u * M_{-x} \widehat{\bar{\psi}}\right)(\xi) \\
& =(2 \pi)^{-2 d} \sum_{|\gamma| \leq m}\left(\left(\widehat{a_{\gamma}} * \widehat{D^{\gamma} u}\right) * M_{-x} \widehat{\bar{\psi}}\right)(\xi) \\
& =(2 \pi)^{-2 d} \sum_{|\gamma| \leq m}\left(\widehat{D^{\gamma} u} *\left(\widehat{a_{\gamma}} * M_{-x} \widehat{\bar{\psi}}\right)\right)(\xi)
\end{aligned}
$$


for all $(x, \xi) \in \mathbb{R}^{2 d}$. We see that for all $t \in \mathbb{R}^{d}$,

$$
\begin{aligned}
\left(\widehat{a_{\gamma}} * M_{-x} \widehat{\bar{\psi}}\right)(t) & =\int \widehat{a_{\gamma}}(t-s) e^{i(-x) \cdot s} \widehat{\bar{\psi}}(s) d s \\
& =e^{i(-x) \cdot t} \int e^{i x \cdot(t-s)} \widehat{a_{\gamma}}(t-s) \widehat{\bar{\psi}}(s) d s \\
& =M_{-x}\left(M_{x} \widehat{a_{\gamma}} * \widehat{\bar{\psi}}\right)(t) .
\end{aligned}
$$

Now, we define $\phi_{x, \gamma} \in \mathcal{S}_{\omega}\left(\mathbb{R}^{d}\right) \backslash\{0\}$ depending on $x \in \mathbb{R}^{d}$ and $\gamma \in \mathbb{N}_{0}^{d}$ with $|\gamma| \leq m$ such that

$$
\widehat{\bar{\phi}}_{x, \gamma}:=M_{x} \widehat{a_{\gamma}} * \widehat{\bar{\psi}}
$$

Then, by (4.3) and using Lemmas 0.32 and 0.33 , we have

$$
\begin{aligned}
V_{\psi}(P(x, D) u)(x, \xi) & =(2 \pi)^{-2 d} \sum_{|\gamma| \leq m}\left(\widehat{D^{\gamma} u} * M_{-x}\left(M_{x} \widehat{a_{\gamma}} * \widehat{\bar{\psi}}\right)\right)(\xi) \\
& =(2 \pi)^{-2 d} \sum_{|\gamma| \leq m}\left(\widehat{D^{\gamma} u} * M_{-x} \widehat{\bar{\phi}}_{x, \gamma}\right)(\xi) \\
& =(2 \pi)^{-d} \sum_{|\gamma| \leq m} V_{\phi_{x, \gamma}}\left(D^{\gamma} u\right)(x, \xi) \\
& =(2 \pi)^{-d} \sum_{|\gamma| \leq m} \sum_{\beta \leq \gamma}\left(\begin{array}{l}
\gamma \\
\beta
\end{array}\right) \xi^{\gamma-\beta} V_{D^{\beta} \phi_{x, \gamma}}(u)(x, \xi) .
\end{aligned}
$$

We show that the set

$$
\mathcal{B}:=\left\{M_{x} \widehat{a_{\gamma}} * \widehat{\bar{\psi}}: x \in \mathbb{R}^{d}, \gamma \in \mathbb{N}_{0}^{d}:|\gamma| \leq m\right\}
$$

is bounded in $\mathcal{S}_{\omega}\left(\mathbb{R}^{d}\right)$. For all $\lambda>0$, we have by (0.1) and the Young inequality,

$$
\begin{aligned}
\left|e^{\lambda \omega(y)}\left(M_{x} \widehat{a_{\gamma}} * \widehat{\bar{\psi}}\right)(y)\right| & =\left|\int e^{\lambda \omega(y)} M_{x} \widehat{a_{\gamma}}(s) \widehat{\bar{\psi}}(y-s) d s\right| \\
& =\left|\int e^{\lambda \omega(y)} e^{i x \cdot s} \widehat{a_{\gamma}}(s) \widehat{\bar{\psi}}(y-s) d s\right| \\
& \leq e^{\lambda L} \int e^{\lambda L \omega(s)}\left|\widehat{a_{\gamma}}(s)\right| e^{\lambda L \omega(y-s)}|\widehat{\bar{\psi}}(y-s)| d s \\
& \leq e^{\lambda L} \max _{|\gamma| \leq m}|| e^{\lambda L \omega(\cdot)} \widehat{a_{\gamma}}(\cdot)\left\|_{L^{1}\left(\mathbb{R}^{d}\right)}\right\| e^{\lambda L \omega(\cdot)} \widehat{\bar{\psi}(\cdot) \|_{L^{\infty}\left(\mathbb{R}^{d}\right)}} .
\end{aligned}
$$


On the other hand, by Lemmas 0.31 and 0.29 we have

$$
M_{x} \widehat{a_{\gamma}} * \widehat{\bar{\psi}}=\widehat{T_{-x} a_{\gamma}} * \widehat{\bar{\psi}}=(2 \pi)^{d}\left(\widehat{T_{-x} a_{\gamma}} \cdot \bar{\psi}\right),
$$

so its Fourier transform satisfies, from (0.34),

$$
\left(\widehat{M_{x} \widehat{a_{\gamma}}} * \widehat{\bar{\psi}}\right)(\eta)=(2 \pi)^{d}\left(\widehat{\widehat{T_{-x} a_{\gamma}}} \cdot \bar{\psi}\right)(\eta)=(2 \pi)^{2 d}\left(T_{-x} a_{\gamma} \cdot \bar{\psi}\right)(-\eta), \quad \eta \in \mathbb{R}^{d} .
$$

Thus, for all $\lambda>0$,

$$
\begin{aligned}
& \left|e^{\lambda \omega(\eta)}\left(\widehat{M_{x} \widehat{a_{\gamma}} *} \widehat{\bar{\psi}}\right)(\eta)\right|=(2 \pi)^{2 d}\left|e^{\lambda \omega(\eta)} T_{-x} a_{\gamma}(-\eta) \bar{\psi}(-\eta)\right| \\
& =(2 \pi)^{2 d}\left|a_{\gamma}(x-\eta) e^{\lambda \omega(-\eta)} \bar{\psi}(-\eta)\right| \\
& \leq(2 \pi)^{2 d} \max _{|\gamma| \leq m}\left\|a_{\gamma}(\cdot)\right\|_{L^{\infty}\left(\mathbb{R}^{d}\right)}\left\|e^{\lambda \omega(\cdot) \bar{\psi}(\cdot)}\right\|_{L^{\infty}\left(\mathbb{R}^{d}\right)} .
\end{aligned}
$$

Formulas (4.7) and (4.8) show that the set given in (4.6) is bounded in $\mathcal{S}_{\omega}\left(\mathbb{R}^{d}\right)$ by $[13$, Theorem $4.8(3)]$.

Since the Fourier transform is an isomorphism in $\mathcal{S}_{\omega}\left(\mathbb{R}^{d}\right)$ (hence the inverse Fourier transform is continuous), the set

$$
\mathcal{F}^{-1}(\mathcal{B})=\{\phi: \widehat{\phi}=f, \quad \text { for some } f \in \mathcal{B}\}
$$

is bounded in $\mathcal{S}_{\omega}\left(\mathbb{R}^{d}\right)$, and therefore

$$
\mathcal{B}^{\prime}:=\{\phi: \widehat{\bar{\phi}}=f, \quad \text { for some } f \in \mathcal{B}\}
$$

is also a bounded set in $\mathcal{S}_{\omega}\left(\mathbb{R}^{d}\right)$. We observe that the function $\phi_{x, \gamma}$ taken in (4.4) belongs to $\mathcal{B}^{\prime}$. We check that

$$
\mathcal{B}^{\prime \prime}:=\left\{D^{\beta} \phi: \phi \in \mathcal{B}^{\prime}, \beta \in \mathbb{N}_{0}^{d}:|\beta| \leq m\right\}
$$

is bounded in $\mathcal{S}_{\omega}\left(\mathbb{R}^{d}\right)$. Let $\phi \in \mathcal{B}^{\prime}$ and let $\lambda>0$. For $|\phi|_{\lambda}$ as in $(0.14)$, we obtain for all $\beta \in \mathbb{N}_{0}^{d}$ with $|\beta| \leq m$, by $(0.11)$,

$$
\begin{aligned}
\left|D^{\beta} \phi\right|_{\lambda} & =\sup _{\alpha \in \mathbb{N}_{0}^{d}} \sup _{x \in \mathbb{R}^{d}}\left|D^{\alpha+\beta} \phi(x)\right| e^{-\lambda \varphi^{*}\left(\frac{|\alpha|}{\lambda}\right)} e^{\lambda \omega(x)} \\
& =e^{\lambda \varphi^{*}\left(\frac{|\beta|}{\lambda}\right)} \sup _{\alpha \in \mathbb{N}_{0}^{d}} \sup _{x \in \mathbb{R}^{d}}\left|D^{\alpha+\beta} \phi(x)\right| e^{-\lambda \varphi^{*}\left(\frac{|\alpha|}{\lambda}\right)} e^{-\lambda \varphi^{*}\left(\frac{|\beta|}{\lambda}\right)} e^{\lambda \omega(x)} \\
& \leq e^{\lambda \varphi^{*}\left(\frac{|\beta|}{\lambda}\right)} \sup _{\alpha \in \mathbb{N}_{0}^{d}} \sup _{x \in \mathbb{R}^{d}}\left|D^{\alpha+\beta} \phi(x)\right| e^{-2 \lambda \varphi^{*}\left(\frac{|\alpha+\beta|}{2 \lambda}\right)} e^{\lambda \omega(x)} \\
& \leq e^{\lambda \varphi^{*}\left(\frac{|\beta|}{\lambda}\right)} \sup _{\delta \in \mathbb{N}_{0}^{d}} \sup _{x \in \mathbb{R}^{d}}\left|D^{\delta} \phi(x)\right| e^{-2 \lambda \varphi^{*}\left(\frac{|\delta|}{2 \lambda}\right)} e^{2 \lambda \omega(x)}=e^{\lambda \varphi^{*}\left(\frac{|\beta|}{\lambda}\right)}|\phi|_{2 \lambda} .
\end{aligned}
$$


Since $e^{\lambda \varphi^{*}\left(\frac{|\beta|}{\lambda}\right)} \leq e^{\lambda \varphi^{*}\left(\frac{m}{\lambda}\right)}$ and $\phi \in \mathcal{B}^{\prime}$, we get

$$
\sup _{|\beta| \leq m} \sup _{\phi \in \mathcal{B}}\left|D^{\beta} \phi\right|_{\lambda}<+\infty
$$

as we wanted.

We show (4.2). To this aim, we denote $z=(x, \xi) \in \mathbb{R}^{2 d}$ and we assume that $0 \neq z_{0} \notin \mathrm{WF}_{\omega}^{\prime}(u)$. Then, there exists $\Gamma \subseteq \mathbb{R}^{2 d} \backslash\{0\}$ an open conic set containing $z_{0}$ such that

$$
\sup _{z \in \Gamma} e^{\lambda \omega(z)}\left|V_{\psi} u(z)\right|<\infty, \quad \lambda>0 .
$$

By Lemma 4.4, for any open conic set $\Gamma^{\prime}$ containing $z_{0}$ with $\overline{\Gamma^{\prime} \cap S_{2 d-1}} \subseteq \Gamma$, we have

$$
\sup _{\beta, \gamma \in \mathbb{N}_{0}^{d}: \beta \leq \gamma,|\gamma| \leq m} \sup _{z \in \mathbb{R}^{d}} e^{\lambda \omega(z)}\left|V_{D^{\beta} \phi_{x, \gamma}}(u)(z)\right|<\infty, \quad \lambda>0 .
$$

By (4.5), for all $\lambda>0$,

$$
\begin{aligned}
& e^{\lambda \omega(z)}\left|V_{\psi}(P(x, D) u)(z)\right| \\
& \quad \leq(2 \pi)^{-d} \sum_{|\gamma| \leq m} \sum_{\beta \leq \gamma}\left(\begin{array}{l}
\gamma \\
\beta
\end{array}\right)\left|\xi^{\gamma-\beta}\right| e^{-L \omega(z)} e^{(\lambda+L) \omega(z)}\left|V_{D^{\beta} \phi_{x, \gamma}}(u)(z)\right| .
\end{aligned}
$$

Since $|\gamma-\beta| \leq|\gamma| \leq m$, we have, by (0.7) and (0.6), that

$$
\left|\xi^{\gamma-\beta}\right| \leq\langle z\rangle^{m} \leq e^{\varphi^{*}(m)} e^{\omega(\langle z\rangle)} \leq e^{\varphi^{*}(m)} e^{L \omega(z)+L}, \quad z=(x, \xi) \in \mathbb{R}^{2 d} .
$$

Therefore

$$
\sup _{(x, \xi) \in \mathbb{R}^{2 d}}\left|\xi^{\gamma-\beta}\right| e^{-L \omega(x, \xi)}<\infty, \quad \beta \leq \gamma,|\gamma| \leq m .
$$

Taking the supremum in (4.10) in $z \in \Gamma^{\prime}$, we obtain by (4.9)

$$
\sup _{z \in \Gamma^{\prime}} e^{\lambda \omega(z)}\left|V_{\psi}(P(x, D) u)(z)\right|<\infty, \quad \lambda>0 .
$$

Hence $z_{0} \notin \mathrm{WF}_{\omega}^{\prime}(P(x, D) u)$ and the proof is complete.

Now, we deal with the Weyl quantization $b^{w}(x, D)$ in $(2.29)$. Since $\mathcal{S}_{\omega}\left(\mathbb{R}^{2 d}\right)$ is nuclear, there exists $K \in \mathcal{S}_{\omega}^{\prime}\left(\mathbb{R}^{4 d}\right)$ such that the operator

$$
V_{\psi} b^{w}(x, D) V_{\psi}^{*}: \mathcal{S}_{\omega}\left(\mathbb{R}^{2 d}\right) \rightarrow \mathcal{S}_{\omega}^{\prime}\left(\mathbb{R}^{2 d}\right)
$$


satisfies

$$
V_{\psi}\left(b^{w}(x, D) V_{\psi}^{*} F\right)\left(y^{\prime}, \eta^{\prime}\right)=(2 \pi)^{d} \int_{\mathbb{R}^{2 d}} K\left(y^{\prime}, \eta^{\prime}, y, \eta\right) F(y, \eta) d y d \eta,
$$

for all $F \in \mathcal{S}_{\omega}\left(\mathbb{R}^{2 d}\right)$, in the sense that

$$
\left\langle V_{\psi} b^{w}(x, D) V_{\psi}^{*} F, G\right\rangle=(2 \pi)^{d}\left\langle K\left(y^{\prime}, \eta^{\prime}, y, \eta\right), G\left(y^{\prime}, \eta^{\prime}\right) F(y, \eta)\right\rangle,
$$

for all $G \in \mathcal{S}_{\omega}\left(\mathbb{R}^{2 d}\right)$. For $u \in \mathcal{S}_{\omega}\left(\mathbb{R}^{d}\right)$ and $\psi \in \mathcal{S}_{\omega}\left(\mathbb{R}^{d}\right)$ with $\|\psi\|_{L^{2}\left(\mathbb{R}^{d}\right)}=1$, we have $V_{\psi} u \in \mathcal{S}_{\omega}\left(\mathbb{R}^{2 d}\right)$ by Theorem 0.28 . For $F=V_{\psi} u$ we have by $(0.32)$ (see [14]),

$$
V_{\psi}\left(b^{w}(x, D) u\right)\left(y^{\prime}, \eta^{\prime}\right)=\int_{\mathbb{R}^{2 d}} K\left(y^{\prime}, \eta^{\prime}, y, \eta\right) V_{\psi} u(y, \eta) d y d \eta, \quad\left(y^{\prime}, \eta^{\prime}\right) \in \mathbb{R}^{2 d}
$$

We analyse the operator (4.11):

Theorem 4.6. Let $b \in \mathrm{GS}_{\rho}^{m, \omega}$ and $\psi \in \mathcal{S}_{\omega}\left(\mathbb{R}^{d}\right)$ such that $\|\psi\|_{L^{2}\left(\mathbb{R}^{d}\right)}=1$. If $u \in \mathcal{S}_{\omega}\left(\mathbb{R}^{d}\right)$ in $(4.11)$, then we have

$$
\begin{aligned}
& K\left(y^{\prime}, \eta^{\prime}, y, \eta\right) \\
& \quad=(2 \pi)^{-2 d} \int_{\mathbb{R}^{2 d}}\left(\int_{\mathbb{R}^{d}} e^{i x \cdot\left(\xi-\eta^{\prime}\right)} e^{i s \cdot(\eta-\xi)} b\left(\frac{x+s}{2}, \xi\right) \overline{\psi\left(x-y^{\prime}\right)} \psi(s-y) d s\right) d \xi d x,
\end{aligned}
$$

for all $\left(y^{\prime}, \eta^{\prime}, y, \eta\right) \in \mathbb{R}^{4 d}$, where $K$ is as in (4.11).

Proof. We consider $V_{\psi}^{*}: \mathcal{S}_{\omega}\left(\mathbb{R}^{2 d}\right) \rightarrow \mathcal{S}_{\omega}\left(\mathbb{R}^{d}\right)$ as in $(0.31)$. By the definition of $V_{\psi}^{*} F, F \in \mathcal{S}_{\omega}\left(\mathbb{R}^{2 d}\right)$, we have for all $\left(y^{\prime}, \eta^{\prime}\right) \in \mathbb{R}^{2 d}$,

$$
\begin{aligned}
V_{\psi}\left(b^{w}\right. & \left.(x, D) V_{\psi}^{*} F\right)\left(y^{\prime}, \eta^{\prime}\right) \\
= & \int_{\mathbb{R}^{d}} e^{-i x \cdot \eta^{\prime}} \overline{\psi\left(x-y^{\prime}\right)} b^{w}(x, D) V_{\psi}^{*} F(x) d x \\
= & (2 \pi)^{-d} \int_{\mathbb{R}^{d}} \int_{\mathbb{R}^{2 d}} e^{-i x \cdot \eta^{\prime}} \overline{\psi\left(x-y^{\prime}\right)} e^{i(x-s) \cdot \xi} b\left(\frac{x+s}{2}, \xi\right) V_{\psi}^{*} F(s) d s d \xi d x \\
= & (2 \pi)^{-d} \int_{\mathbb{R}^{d}} \int_{\mathbb{R}^{2 d}} \int_{\mathbb{R}^{2 d}} e^{-i x \cdot \eta^{\prime}} \overline{\psi\left(x-y^{\prime}\right)} e^{i(x-s) \cdot \xi} b\left(\frac{x+s}{2}, \xi\right) \times \\
& \times F(y, \eta) e^{i s \cdot \eta} \psi(s-y) d y d \eta d s d \xi d x .
\end{aligned}
$$

We can assume without losing generality that $m \geq 0$. To show the result, we need to apply Fubini's theorem, first to the variables $y, \eta, s$. To this, we 
estimate the modulus of the integrand above: since $\psi \in \mathcal{S}_{\omega}\left(\mathbb{R}^{d}\right), b \in \mathrm{GS}_{\rho}^{m, \omega}$, and $F \in \mathcal{S}_{\omega}\left(\mathbb{R}^{2 d}\right)$, there are $C, C_{m}>0$ and for all $\lambda_{1}, \lambda_{2}>0$ there exist $C_{\lambda_{1}}, C_{\lambda_{2}}>0$ satisfying, from formulas (0.2), (0.3), and (0.1),

$$
\begin{aligned}
& \left.\mid \overline{\psi(x}-y^{\prime}\right) b\left(\frac{x+s}{2}, \xi\right) F(y, \eta) \psi(s-y) \mid \\
& \quad \leq C C_{m} e^{m \omega\left(\frac{x+s}{2}, \xi\right)} C_{\lambda_{1}} e^{-\lambda_{1} \omega(y, \eta)} C_{\lambda_{2}} e^{-\lambda_{2} \omega(s-y)} \\
& \quad \leq C_{\lambda_{1}, \lambda_{2}} e^{m L \omega(x)} e^{m L \omega(s)} e^{m L \omega(\xi)} e^{m L} e^{-\left(\lambda_{1} / 2\right)(\omega(y)+\omega(\eta))} e^{-\left(\lambda_{2} / L\right) \omega(s)} e^{\lambda_{2} \omega(y)} e^{\lambda_{2}},
\end{aligned}
$$

for $C_{\lambda_{1}, \lambda_{2}}=C C_{m} C_{\lambda_{1}} C_{\lambda_{2}}>0$, where the last function belongs to $L^{1}\left(\mathbb{R}_{y, \eta, s}^{3 d}\right)$ if we choose $\lambda_{2}>m L^{2}$ (then, the integral depending on $s$ converges by property $(\gamma)$ of the weight) and $\lambda_{1}>2 \lambda_{2}$ (then, the integrals depending on $y$ and $\eta$ converge by property $(\gamma)$ ). Thus, by Fubini's theorem,

$$
\begin{aligned}
V_{\psi}\left(b^{w}(x, D) V_{\psi}^{*} F\right)\left(y^{\prime}, \eta^{\prime}\right)= & (2 \pi)^{-d} \int_{\mathbb{R}^{d}} \int_{\mathbb{R}^{d}} \int_{\mathbb{R}^{2 d}} e^{i x \cdot \xi} F(y, \eta) \overline{\psi\left(x-y^{\prime}\right)} e^{-i x \cdot \eta^{\prime}} \times \\
& \times\left(\int_{\mathbb{R}^{d}} e^{i s \cdot(\eta-\xi)} b\left(\frac{x+s}{2}, \xi\right) \psi(s-y) d s\right) d y d \eta d \xi d x .
\end{aligned}
$$

Now, we want to use Fubini's theorem in $d y d \eta d \xi d x$. To that aim, we need some preparation for

$$
I(y, \eta, \xi, x):=\int_{\mathbb{R}^{d}} e^{i s \cdot(\eta-\xi)} b\left(\frac{x+s}{2}, \xi\right) \psi(s-y) d s .
$$

As $b \in \mathrm{GS}_{\rho}^{m, \omega}$ and $\psi \in \mathcal{S}_{\omega}\left(\mathbb{R}^{d}\right)$, there is $C>0$ and for all $\lambda>0$ there exists $C_{\lambda}>0$ with (by (0.3), (0.2), and (0.1))

$$
\begin{aligned}
\left|b\left(\frac{x+s}{2}, \xi\right) \psi(s-y)\right| & \leq C e^{m \omega\left(\frac{x+s}{2}, \xi\right)} C_{\lambda} e^{-\lambda \omega(s-y)} \\
& \leq C C_{\lambda} e^{m L \omega(x)} e^{m L \omega(s)} e^{m L \omega(\xi)} e^{m L} e^{-(\lambda / L) \omega(s)} e^{\lambda \omega(y)} e^{\lambda},
\end{aligned}
$$

which belongs to $L^{1}\left(\mathbb{R}_{s}^{d}\right)$ if $\lambda>m L^{2}$.

We assume $|\eta-\xi|_{\infty}:=\max _{1 \leq h \leq d}\left|\eta_{h}-\xi_{h}\right|=\left|\eta_{j}-\xi_{j}\right| \geq 1$, for some $1 \leq j \leq d$. Let $s=\left(s_{1}, \ldots, s_{d}\right) \in \mathbb{R}^{d}$. For any $N \in \mathbb{N}_{0}$, we integrate by parts in (4.14) 
with the variable $s_{j}$ as follows:

$$
\begin{aligned}
|I| & =\left|\int_{\mathbb{R}^{d}} \frac{1}{\left(\eta_{j}-\xi_{j}\right)^{N}}\left(D_{s_{j}}^{N} e^{i s \cdot(\eta-\xi)}\right) b\left(\frac{x+s}{2}, \xi\right) \psi(s-y) d s\right| \\
& =\left|\int_{\mathbb{R}^{d}} \frac{(-1)^{N}}{\left(\eta_{j}-\xi_{j}\right)^{N}} e^{i s \cdot(\eta-\xi)} D_{s_{j}}^{N}\left(b\left(\frac{x+s}{2}, \xi\right) \psi(s-y)\right) d s\right| \\
& \leq \frac{1}{\left|\eta_{j}-\xi_{j}\right|^{N}} \sum_{k=0}^{N}\left(\begin{array}{c}
N \\
k
\end{array}\right) \int_{\mathbb{R}^{d}}\left|D_{s_{j}}^{N-k} b\left(\frac{x+s}{2}, \xi\right)\right|\left|D_{s_{j}}^{k} \psi(s-y)\right| d s .
\end{aligned}
$$

We observe that $|\eta-\xi| \leq \sqrt{d}|\eta-\xi|_{\infty}=\sqrt{d}\left|\eta_{j}-\xi_{j}\right|$. We put $p \in \mathbb{N}$ so that $2 \sqrt{d} \leq e^{p}$, and since $b \in \mathrm{GS}_{\rho}^{m, \omega}$ and $\psi \in \mathcal{S}_{\omega}\left(\mathbb{R}^{d}\right)$, we obtain that for all $n \in \mathbb{N}$ there exist $C_{n}, C_{n}^{\prime}>0$ such that, by (0.11), (0.2), and (0.1) (since $\left.\sum_{k=0}^{N}\left(\begin{array}{l}N \\ k\end{array}\right)=2^{N}\right)$,

$$
\begin{aligned}
|I| \leq & \frac{(\sqrt{d})^{N}}{|\eta-\xi|^{N}} \sum_{k=0}^{N}\left(\begin{array}{c}
N \\
k
\end{array}\right) \int_{\mathbb{R}^{d}} C_{n}\left\langle\left(\frac{x+s}{2}, \xi\right)\right\rangle^{-\rho(N-k)} \times \\
& \times e^{(n+1) L^{p} \rho \varphi^{*}\left(\frac{N-k}{(n+1) L^{p}}\right)} e^{m \omega\left(\frac{x+s}{2}, \xi\right)} C_{n}^{\prime} e^{(n+1) L^{p} \varphi^{*}\left(\frac{k}{(n+1) L^{p}}\right)} e^{-\left(m L^{2}+L\right) \omega(s-y)} d s \\
\leq & C_{n} C_{n}^{\prime} \frac{(\sqrt{d})^{N}}{|\eta-\xi|^{N}} e^{(n+1) L^{p} \varphi^{*}\left(\frac{N}{(n+1) L^{p}}\right)} \sum_{k=0}^{N}\left(\begin{array}{c}
N \\
k
\end{array}\right) \int_{\mathbb{R}^{d}} e^{m \omega\left(\frac{x+s}{2}, \xi\right)} e^{-\left(m L^{2}+L\right) \omega(s-y)} d s \\
\leq & C_{n} C_{n}^{\prime} \frac{(2 \sqrt{d})^{N}}{|\eta-\xi|^{N}} e^{(n+1) L^{p} \varphi^{*}\left(\frac{N}{(n+1) L^{p}}\right)} \times \\
& \times \int_{\mathbb{R}^{d}} e^{m L \omega(x)+m L \omega(s)+m L \omega(\xi)} e^{m L} e^{-\left(\left(m L^{2}+L\right) / L\right) \omega(s)+\left(m L^{2}+L\right) \omega(y)} e^{m L^{2}+L} d s .
\end{aligned}
$$

By the choice of $p \in \mathbb{N}$, we have by $(0.10)$,

$$
\begin{aligned}
|I| \leq & C_{n} C_{n}^{\prime} e^{m L} e^{m L^{2}+L} e^{(n+1) \sum_{j=1}^{p} L^{j}}|\eta-\xi|^{-N} e^{(n+1) \varphi^{*}\left(\frac{N}{n+1}\right)} \times \\
& \times e^{m L \omega(x)} e^{m L \omega(\xi)} e^{\left(m L^{2}+L\right) \omega(y)} \int_{\mathbb{R}^{d}} e^{-\omega(s)} d s .
\end{aligned}
$$

The last integral is convergent by property $(\gamma)$ of Definition 0.3 . Take the infimum on $N \in \mathbb{N}_{0}$, and then use (0.8) to obtain that for each $n \in \mathbb{N}$ there exists $C_{n}^{\prime \prime}>0$ such that, by $(0.7)$ and $(0.1)$,

$$
\begin{aligned}
|I| & \leq C_{n}^{\prime \prime} e^{-(n+1) \omega(\eta-\xi)+\log |\eta-\xi|} e^{m L \omega(x)} e^{m L \omega(\xi)} e^{\left(m L^{2}+L\right) \omega(y)} \\
& \leq C_{n}^{\prime \prime} e^{\varphi^{*}(1)} e^{-n \omega(\eta-\xi)} e^{m L \omega(x)} e^{m L \omega(\xi)} e^{\left(m L^{2}+L\right) \omega(y)} \\
& \leq C_{n}^{\prime \prime} e^{\varphi^{*}(1)} e^{n} e^{(m L-n / L) \omega(\xi)} e^{n \omega(\eta)} e^{m L \omega(x)} e^{\left(m L^{2}+L\right) \omega(y)} .
\end{aligned}
$$


Thus, since $F \in \mathcal{S}_{\omega}\left(\mathbb{R}^{2 d}\right)$ and $\psi \in \mathcal{S}_{\omega}\left(\mathbb{R}^{d}\right)$, for all $x, y, y^{\prime}, \eta, \xi \in \mathbb{R}^{d}$ satisfying $|\eta-\xi|_{\infty} \geq 1$, we have that for all $\lambda, \lambda_{1}, \lambda_{2}>0$ there are $C_{\lambda}, C_{\lambda_{1}}, C_{\lambda_{2}}>0$ with (by $(0.1))$,

$$
\begin{aligned}
\mid F(y, & \eta \overline{\psi\left(x-y^{\prime}\right)} I \mid \\
\leq & C_{\lambda_{1}} e^{-\lambda_{1} \omega(y, \eta)} C_{\lambda_{2}} e^{-\lambda_{2} \omega\left(x-y^{\prime}\right)} C_{\lambda} e^{(m L-\lambda / L) \omega(\xi)} e^{\lambda \omega(\eta)} e^{m L \omega(x)} e^{\left(m L^{2}+L\right) \omega(y)} \\
\leq & C_{\lambda_{1}} e^{-\left(\lambda_{1} / 2\right) \omega(y)} e^{-\left(\lambda_{1} / 2\right) \omega(\eta)} C_{\lambda_{2}} e^{-\left(\lambda_{2} / L\right) \omega(x)} e^{\lambda_{2} \omega\left(y^{\prime}\right)} e^{\lambda_{2}} \times \\
& \times C_{\lambda} e^{(m L-\lambda / L) \omega(\xi)} e^{\lambda \omega(\eta)} e^{m L \omega(x)} e^{\left(m L^{2}+L\right) \omega(y)} \\
= & C_{\lambda_{,}, \lambda_{1}, \lambda_{2}} e^{\left(m L^{2}+L-\lambda_{1} / 2\right) \omega(y)} e^{\left(\lambda-\lambda_{1} / 2\right) \omega(\eta)} e^{(m L-\lambda / L) \omega(\xi)} e^{\left(m L-\lambda_{2} / L\right) \omega(x)} e^{\lambda_{2} \omega\left(y^{\prime}\right)},
\end{aligned}
$$

where $C_{\lambda, \lambda_{1}, \lambda_{2}}=C_{\lambda_{1}} C_{\lambda_{2}} C_{\lambda} e^{\lambda_{2}}>0$. We observe that (4.15) is estimated by a function in $L^{1}\left(\mathbb{R}_{y, \eta, \xi, x}^{4 d}\right)$ if $\lambda>m L^{2}$ (the integral in $d \xi$ converges), $\lambda_{2}>m L^{2}$ (the integral in $d x$ converges), and $\lambda_{1}>\max \left\{2\left(m L^{2}+L\right), 2 \lambda\right\}$ (the integrals in $d y$ and $d \eta$ converge).

On the other hand, if $|\eta-\xi|_{\infty} \leq 1$, then $|\xi|-|\eta| \leq|\eta-\xi| \leq \sqrt{d}|\eta-\xi|_{\infty} \leq \sqrt{d}$, so $|\xi| \leq|\eta|+\sqrt{d}$. Hence by $(0.1)$,

$$
\omega(\xi) \leq \omega(|\eta|+\sqrt{\bar{d}}) \leq L \omega(\eta)+L \omega(\sqrt{d})+L .
$$

Then, as $F \in \mathcal{S}_{\omega}\left(\mathbb{R}^{2 d}\right), \psi \in \mathcal{S}_{\omega}\left(\mathbb{R}^{d}\right)$ and $b \in \mathrm{GS}_{\rho}^{m, \omega}$, there exists $C>0$ and for all $\lambda, \lambda_{1}, \lambda_{2}>0$ there exist $C_{\lambda}, C_{\lambda_{1}}, C_{\lambda_{2}}>0$ satisfying

$$
\begin{aligned}
\mid F(y, & \eta \overline{\psi\left(x-y^{\prime}\right)} b\left(\frac{x+s}{2}, \xi\right) \psi(s-y) \mid \\
\leq & C_{\lambda} e^{-\lambda \omega(y, \eta)} C_{\lambda_{1}} e^{-\lambda_{1} \omega\left(x-y^{\prime}\right)} C e^{m \omega\left(\frac{x+s}{2}, \xi\right)} C_{\lambda_{2}} e^{-\lambda_{2} \omega(s-y)} \\
\leq & C_{\lambda} e^{-(\lambda / 2) \omega(y)} e^{-(\lambda / 2) \omega(\eta)} C_{\lambda_{1}} e^{-\left(\lambda_{1} / L\right) \omega(x)} e^{\lambda_{1} \omega\left(y^{\prime}\right)} e^{\lambda_{1}} \times \\
& \times C e^{m L \omega(x)} e^{m L \omega(s)} e^{(m L+1) \omega(\xi)} e^{-\omega(\xi)} e^{m L} C_{\lambda_{2}} e^{-\left(\lambda_{2} / L\right) \omega(s)} e^{\lambda_{2} \omega(y)} e^{\lambda_{2}} .
\end{aligned}
$$

Then, for all $x, y, y^{\prime}, \eta, \xi \in \mathbb{R}^{d}$ satisfying $|\eta-\xi|_{\infty} \leq 1$, by (4.16), we get for $C_{\lambda, \lambda_{1}, \lambda_{2}}=C C_{\lambda} C_{\lambda_{1}} C_{\lambda_{2}} e^{\lambda_{1}} e^{\lambda_{2}} e^{m L} e^{(m L+1)(L \omega(\sqrt{d})+L)}>0$,

$$
\begin{aligned}
\mid F(y, \eta) & \overline{\psi\left(x-y^{\prime}\right)} b\left(\frac{x+s}{2}, \xi\right) \psi(s-y) \mid \\
\leq & C_{\lambda, \lambda_{1}, \lambda_{2}} e^{\left(m L-\lambda_{2} / L\right) \omega(s)} e^{\left(\lambda_{2}-\lambda / 2\right) \omega(y)} \times \\
& \times e^{\left(m L^{2}+L-\lambda / 2\right) \omega(\eta)} e^{-\omega(\xi)} e^{\left(m L-\lambda_{1} / L\right) \omega(x)} e^{\lambda_{1} \omega\left(y^{\prime}\right)},
\end{aligned}
$$

which is estimated by a function in $L^{1}\left(\mathbb{R}_{s, y, \eta, \xi, x}^{5 d}\right)$ if $\lambda_{2}>m L^{2}$ (the integral depending on $s$ converges), $\lambda>\max \left\{2 \lambda_{2}, 2 m L^{2}+2 L\right\}$ (the integrals depending 
on $y$ and $\eta$ converge), and $\lambda_{1}>m L^{2}$ (the integral depending on $x$ converges). From (4.15) and (4.17), we can use Fubini's theorem in (4.13) in $d y d \eta d \xi d x$ :

$$
\begin{aligned}
& V_{\psi}\left(b^{w}(x, D) V_{\psi}^{*} F\right)\left(y^{\prime}, \eta^{\prime}\right) \\
& =(2 \pi)^{-d} \int_{\mathbb{R}^{2 d}}\left(\int_{\mathbb{R}^{3 d}} e^{i x \cdot\left(\xi-\eta^{\prime}\right)} e^{i s \cdot(\eta-\xi)} b\left(\frac{x+s}{2}, \xi\right) \overline{\psi\left(x-y^{\prime}\right)} \psi(s-y) d s d \xi d x\right) \times \\
& \quad \times F(y, \eta) d y d \eta .
\end{aligned}
$$

For $u \in \mathcal{S}_{\omega}\left(\mathbb{R}^{d}\right)$, put $F=V_{\psi} u$. From $(0.32)$, since $\|\psi\|_{L^{2}\left(\mathbb{R}^{d}\right)}=1$,

$$
V_{\psi}^{*} F=V_{\psi}^{*} V_{\psi} u=(2 \pi)^{d} u .
$$

Hence

$$
V_{\psi}\left(b^{w}(x, D) u\right)\left(y^{\prime}, \eta^{\prime}\right)=\int_{\mathbb{R}^{2 d}} K\left(y^{\prime}, \eta^{\prime}, y, \eta\right) V_{\psi} u(y, \eta) d y d \eta,
$$

for all $\left(y^{\prime}, \eta^{\prime}\right) \in \mathbb{R}^{2 d}$, where the kernel $K\left(y^{\prime}, \eta^{\prime}, y, \eta\right)$ is as in (4.12).

Under the assumptions in Theorem 4.6, we estimate the kernel (4.12) as in [14, Proposition 4.4], for our classes of global symbols.

Theorem 4.7. Let $b \in \mathrm{GS}_{\rho}^{m, \omega}$ and $\psi \in \mathcal{S}_{\omega}\left(\mathbb{R}^{d}\right)$ with $\|\psi\|_{L^{2}\left(\mathbb{R}^{d}\right)}=1$. If $u \in$ $\mathcal{S}_{\omega}\left(\mathbb{R}^{d}\right)$ and $K\left(y^{\prime}, \eta^{\prime}, y, \eta\right)$ is as in $(4.12)$, then for all $\lambda>0$ there exist $C_{\lambda}, \mu_{\lambda}>$ 0 such that

$$
\left|K\left(y^{\prime}, \eta^{\prime}, y, \eta\right)\right| \leq C_{\lambda} e^{-\lambda \omega\left(y-y^{\prime}\right)} e^{-\lambda \omega\left(\eta-\eta^{\prime}\right)} e^{\mu_{\lambda} \omega\left(\eta^{\prime}\right)} e^{\max \left\{0, m L^{2}\right\}\left(\omega\left(y^{\prime}\right)+\omega(y)\right)}
$$

for all $\left(y^{\prime}, \eta^{\prime}, y, \eta\right) \in \mathbb{R}^{4 d}$.

Moreover, if $b(z)=0$ for $z \in \Gamma \backslash \overline{B(0, R)}$ for an open conic set $\Gamma \subseteq \mathbb{R}^{2 d} \backslash\{0\}$ and for some $R>0$, then for every open conic set $\Gamma^{\prime} \subseteq \mathbb{R}^{2 d} \backslash\{0\}$ such that $\overline{\Gamma^{\prime} \cap S_{2 d-1}} \subseteq \Gamma$ (where $S_{2 d-1}$ denotes the unit sphere in $\overline{\mathbb{R}}^{2 d}$ ) we have that for all $\lambda>0$ there exists $C_{\lambda}>0$ such that

$$
\left|K\left(y^{\prime}, \eta^{\prime}, y, \eta\right)\right| \leq C_{\lambda} e^{-\lambda \omega\left(y-y^{\prime}\right)} e^{-\lambda \omega\left(\eta-\eta^{\prime}\right)} e^{-2 \lambda \omega\left(y^{\prime}\right)} e^{-2 \lambda \omega\left(\eta^{\prime}\right)}
$$

for all $\left(y^{\prime}, \eta^{\prime}\right) \in \Gamma^{\prime},(y, \eta) \in \mathbb{R}^{2 d}$.

Proof. We assume without losing generality that $m>0$. We make the change of variables in the kernel (4.12)

$$
x^{\prime}=x-y^{\prime}, \quad s^{\prime}=s-y .
$$


By abuse of notation, we write $x$ and $s$ for $x^{\prime}$ and $s^{\prime}$. By Theorem 4.6, we have

$$
\begin{aligned}
K\left(y^{\prime}, \eta^{\prime}, y, \eta\right)= & (2 \pi)^{-2 d} \int_{\mathbb{R}^{3 d}} e^{i\left(x+y^{\prime}\right) \cdot\left(\xi-\eta^{\prime}\right)+i(s+y) \cdot(\eta-\xi)} \times \\
& \times b\left(\frac{x+y^{\prime}+s+y}{2}, \xi\right) \overline{\psi(x)} \psi(s) d s d x d \xi \\
= & (2 \pi)^{-2 d} e^{-i y^{\prime} \cdot \eta^{\prime}+i y \cdot \eta} \int_{\mathbb{R}^{3 d}} e^{i s \cdot(\eta-\xi)} e^{i x \cdot\left(\xi-\eta^{\prime}\right)} e^{i \xi \cdot\left(y^{\prime}-y\right)} \times \\
& \times b\left(\frac{x+y^{\prime}+s+y}{2}, \xi\right) \overline{\psi(x)} \psi(s) d s d x d \xi
\end{aligned}
$$

Take $\ell, h \in \mathbb{N}, k \in \mathbb{N}_{0}$. For the powers of the ultradifferential operator $G(D)$, $G^{\ell}(D)$ and $G^{h}(D)$, we use (1.7), and we obtain

$$
\begin{aligned}
e^{i\left(s \cdot(\eta-\xi)+x \cdot\left(\xi-\eta^{\prime}\right)+\xi \cdot\left(y^{\prime}-y\right)\right)} & \frac{1}{G^{\ell}(\xi-\eta)} G^{\ell}\left(-D_{s}\right)\left[e^{i\left(s \cdot(\eta-\xi)+x \cdot\left(\xi-\eta^{\prime}\right)+\xi \cdot\left(y^{\prime}-y\right)\right)}\right] \\
= & \frac{1}{G^{\ell}(\xi-\eta) G^{h}\left(\eta^{\prime}-\xi\right)} G^{\ell}\left(-D_{s}\right) e^{i s \cdot(\eta-\xi)} G^{h}\left(-D_{x}\right)\left[e^{i\left(x \cdot\left(\xi-\eta^{\prime}\right)+\xi \cdot\left(y^{\prime}-y\right)\right)}\right] \\
= & \frac{1}{G^{\ell}(\xi-\eta) G^{h}\left(\eta^{\prime}-\xi\right)\left\langle y-y^{\prime}\right\rangle^{2 k}} \times \\
& \times G^{\ell}\left(-D_{s}\right) e^{i s \cdot(\eta-\xi)} G^{h}\left(-D_{x}\right) e^{i x \cdot\left(\xi-\eta^{\prime}\right)}\left(1-\Delta_{\xi}\right)^{k} e^{i \xi \cdot\left(y^{\prime}-y\right)},
\end{aligned}
$$

where $\Delta_{\xi}$ denotes the Laplacian in the variable $\xi$. We use this formula into (4.20) and then integrate by parts to write

$$
\left|K\left(y^{\prime}, \eta^{\prime}, y, \eta\right)\right|=(2 \pi)^{-2 d}\left\langle y-y^{\prime}\right\rangle^{-2 k}\left|\int_{\mathbb{R}^{3 d}} e^{i \xi \cdot\left(y^{\prime}-y\right)} \lambda_{\ell, h, k}\left(y^{\prime}, \eta^{\prime}, y, \eta, s, x, \xi\right) d s d x d \xi\right|
$$

with

$$
\begin{aligned}
\lambda_{\ell, h, k}\left(y^{\prime}, \eta^{\prime}, y, \eta, s, x, \xi\right)= & \left(1-\Delta_{\xi}\right)^{k}\left[G^{-\ell}(\xi-\eta) G^{-h}\left(\eta^{\prime}-\xi\right) e^{i x \cdot\left(\xi-\eta^{\prime}\right)} e^{i s \cdot(\eta-\xi)} \times\right. \\
& \left.\times G^{h}\left(D_{x}\right) G^{\ell}\left(D_{s}\right)\left\{b\left(\frac{x+y^{\prime}+s+y}{2}, \xi\right) \overline{\psi(x)} \psi(s)\right\}\right] .
\end{aligned}
$$

Since $\psi \in \mathcal{S}_{\omega}\left(\mathbb{R}^{d}\right)$ and $b \in \mathrm{GS}_{\rho}^{m, \omega}$, it clearly follows that we can integrate by parts in $d s$ and $d x$. To check if we can integrate by parts in $d \xi$, we estimate 
$\left|\lambda_{\ell, h, k}\right|$ by a function in $L^{1}\left(\mathbb{R}_{s, x}^{2 d}\right)$. Indeed, for $\xi=\left(\xi_{1}, \ldots, \xi_{d}\right) \in \mathbb{R}^{d}$,

$$
\begin{aligned}
\left|\lambda_{\ell, h, k}\right|= & \mid\left(1+D_{\xi_{1}}^{2}+\cdots+D_{\xi_{d}}^{2}\right)^{k}\left[G^{-\ell}(\xi-\eta) G^{-h}\left(\eta^{\prime}-\xi\right) e^{i x \cdot\left(\xi-\eta^{\prime}\right)} e^{i s \cdot(\eta-\xi)} \times\right. \\
& \left.\times G^{h}\left(D_{x}\right) G^{\ell}\left(D_{s}\right)\left\{b\left(\frac{x+y^{\prime}+s+y}{2}, \xi\right) \overline{\psi(x)} \psi(s)\right\}\right] \mid \\
\leq & \sum_{j_{1}+\cdots+j_{d}+j_{d+1}=k} \mid \frac{k !}{j_{1} ! \cdots j_{d} ! j_{d+1} !} D_{\xi_{1}}^{2 j_{1}} \cdots D_{\xi_{d}}^{2 j_{d}}\left[G^{-\ell}(\xi-\eta) G^{-h}\left(\eta^{\prime}-\xi\right) \times\right. \\
& \left.\times e^{i x \cdot\left(\xi-\eta^{\prime}\right)} e^{i s \cdot(\eta-\xi)} G^{h}\left(D_{x}\right) G^{\ell}\left(D_{s}\right)\left\{b\left(\frac{x+y^{\prime}+s+y}{2}, \xi\right) \overline{\psi(x)} \psi(s)\right\}\right] \mid \\
= & \sum_{j^{\prime}=0}^{k}\left(\begin{array}{l}
k \\
j^{\prime}
\end{array}\right)_{j_{1}+\cdots+j_{d}=k-j^{\prime}} \sum_{j_{1} ! \cdots j_{d} !} D_{\xi_{1}}^{2 j_{1}} \cdots D_{\xi_{d}}^{2 j_{d}}\left[G^{-\ell}(\xi-\eta) G^{-h}\left(\eta^{\prime}-\xi\right) \times\right. \\
& \left.\times e^{i x \cdot\left(\xi-\eta^{\prime}\right)} e^{i s \cdot(\eta-\xi)} G^{h}\left(D_{x}\right) G^{\ell}\left(D_{s}\right)\left\{b\left(\frac{x+y^{\prime}+s+y}{2}, \xi\right) \overline{\psi(x)} \psi(s)\right\}\right] \mid .
\end{aligned}
$$

Then, for $j=\left(j_{1}, \ldots, j_{d}\right) \in \mathbb{N}_{0}^{d}$, we have $D_{\xi_{1}}^{2 j_{1}} \cdots D_{\xi_{d}}^{2 j_{d}}=D_{\xi}^{2 j}$. So, by Leibniz rule,

$$
\begin{aligned}
\left|\lambda_{\ell, h, k}\right| \leq & \sum_{j^{\prime}=0}^{k}\left(\begin{array}{c}
k \\
j^{\prime}
\end{array}\right) \sum_{|j|=k-j^{\prime}} \frac{\left(k-j^{\prime}\right) !}{j_{1} ! \cdots j_{d} !} \sum_{\sigma_{1}+\cdots+\sigma_{5}=2 j} \frac{(2 j) !}{\sigma_{1} ! \cdots \sigma_{5} !} \times \\
& \times\left|D_{\xi}^{\sigma_{1}} G^{-\ell}(\xi-\eta)\right|\left|D_{\xi}^{\sigma_{2}} G^{-h}\left(\eta^{\prime}-\xi\right)\right|\left|D_{\xi}^{\sigma_{3}} e^{i x \cdot\left(\xi-\eta^{\prime}\right)}\right|\left|D_{\xi}^{\sigma_{4}} e^{i s \cdot(\eta-\xi)}\right| \times \\
& \times\left|D_{\xi}^{\sigma_{5}} G^{h}\left(D_{x}\right) G^{\ell}\left(D_{s}\right)\left\{b\left(\frac{x+y^{\prime}+s+y}{2}, \xi\right) \overline{\psi(x)} \psi(s)\right\}\right| \\
\leq & \sum_{j^{\prime}=0}^{k}\left(\begin{array}{c}
k \\
j^{\prime}
\end{array}\right) \sum_{|j|=k-j^{\prime}} \frac{\left(2\left(k-j^{\prime}\right)\right) !}{\left(2 j_{1}\right) ! \cdots\left(2 j_{d}\right) !} \sum_{\sigma_{1}+\cdots+\sigma_{5}=2 j} \frac{\left(2 j_{1}\right) ! \cdots\left(2 j j_{d}\right) !}{\sigma_{1} ! \cdots \sigma_{5} !} \times \\
& \times\left|D_{\xi}^{\sigma_{1}} G^{-\ell}(\xi-\eta)\right|\left|D_{\xi}^{\sigma_{2}} G^{-h}\left(\eta^{\prime}-\xi\right)\right||x|^{\left|\sigma_{3}\right|}|s|^{\left|\sigma_{4}\right|} \times \\
& \times\left|D_{\xi}^{\sigma_{5}} G^{h}\left(D_{x}\right) G^{\ell}\left(D_{s}\right)\left\{b\left(\frac{x+y^{\prime}+s+y}{2}, \xi\right) \overline{\psi(x)} \psi(s)\right\}\right| \\
\leq & \sum_{j^{\prime}=0}^{k}\left(\begin{array}{l}
k^{\prime} \\
j^{\prime}
\end{array}\right) \quad \sum_{\left|\sigma_{1}+\cdots+\sigma_{5}\right|=2\left(k-j^{\prime}\right)} \frac{\left(2\left(k-j^{\prime}\right)\right) !}{\sigma_{1} ! \cdots \sigma_{5} !} \times \\
& \times\left|D_{\xi}^{\sigma_{1}} G^{-\ell}(\xi-\eta)\right|\left|D_{\xi}^{\sigma_{2}} G^{-h}\left(\eta^{\prime}-\xi\right)\right||x|^{\left|\sigma_{3}\right|}|s|^{\left|\sigma_{4}\right|} \times \\
& \times\left|D_{\xi}^{\sigma_{5}} G^{h}\left(D_{x}\right) G^{\ell}\left(D_{s}\right)\left\{b\left(\frac{x+y^{\prime}+s+y}{2}, \xi\right) \overline{\psi(x)} \psi(s)\right\}\right| .
\end{aligned}
$$


We take $M \in \mathbb{N}_{0}$ to be determined later. By Corollary 0.20 and (0.12), we deduce that there exist $C_{1}, C_{3}, C_{M}>0$ such that

$$
\begin{aligned}
& \left|D_{\xi}^{\sigma_{1}} G^{-\ell}(\xi-\eta)\right| \leq C_{1}^{\ell} C_{M} e^{(M+1) L^{2} \varphi^{*}\left(\frac{\left|\sigma_{1}\right|}{(M+1) L^{2}}\right)} e^{-\ell C_{3} \omega(\xi-\eta)} \\
& \left|D_{\xi}^{\sigma_{2}} G^{-h}\left(\eta^{\prime}-\xi\right)\right| \leq C_{1}^{h} C_{M} e^{(M+1) L^{2} \varphi^{*}\left(\frac{\left|\sigma_{2}\right|}{(M+1) L^{2}}\right)} e^{-h C_{3} \omega\left(\eta^{\prime}-\xi\right)} .
\end{aligned}
$$

By (0.7), we have

$$
\begin{aligned}
& |x|^{\left|\sigma_{3}\right|} \leq e^{(M+1) L^{2} \varphi^{*}\left(\frac{\left|\sigma_{3}\right|}{(M+1) L^{2}}\right)} e^{(M+1) L^{2} \omega(x)} \\
& |s|^{\left|\sigma_{4}\right|} \leq e^{(M+1) L^{2} \varphi^{*}\left(\frac{\left|\sigma_{4}\right|}{(M+1) L^{2}}\right)} e^{(M+1) L^{2} \omega(s)} .
\end{aligned}
$$

Now, by Corollary 0.23 , there exists $C_{4}>0$ such that

$$
\begin{aligned}
\left|D_{\xi}^{\sigma_{5}} G^{h}\left(D_{x}\right) G^{\ell}\left(D_{s}\right)\left\{b\left(\frac{x+y^{\prime}+s+y}{2}, \xi\right) \overline{\psi(x)} \psi(s)\right\}\right| \\
\leq \sum_{\delta, \tau \in \mathbb{N}_{0}^{d}} e^{h C_{4}} e^{-h C_{4} \varphi^{*}\left(\frac{|\delta|}{h C_{4}}\right)} e^{\ell C_{4}} e^{-\ell C_{4} \varphi^{*}}\left(\frac{|\tau|}{\ell C_{4}}\right) \sum_{\substack{\delta_{1}+\delta_{2}=\delta \\
\tau_{1}+\tau_{2}=\tau}} \frac{\delta !}{\delta_{1} ! \delta_{2} !} \frac{\tau !}{\tau_{1} ! \tau_{2} !} \times \\
\quad \times\left|D_{x}^{\delta_{1}} D_{s}^{\tau_{1}} D_{\xi}^{\sigma 5} b\left(\frac{x+y^{\prime}+s+y}{2}, \xi\right)\right|\left|D_{x}^{\delta_{2}} \overline{\psi(x)}\right|\left|D_{s}^{\tau_{2}} \psi(s)\right| .
\end{aligned}
$$

As $b \in \mathrm{GS}_{\rho}^{m, \omega}$, there exists $C_{M}^{\prime}>0$ such that

$$
\left|D_{x}^{\delta_{1}} D_{s}^{\tau_{1}} D_{\xi}^{\sigma_{5}} b\left(\frac{x+y^{\prime}+s+y}{2}, \xi\right)\right| \leq C_{M}^{\prime} e^{4(M+1) L^{2} \rho \varphi^{*}\left(\frac{\left|\delta_{1}+\tau_{1}+\sigma_{5}\right|}{4(M+1) L^{2}}\right)} e^{m \omega\left(\frac{x+y^{\prime}+s+y}{2}, \xi\right)} .
$$

From (0.3) and property $(\alpha)$ we have

$$
\begin{aligned}
e^{m \omega\left(\frac{x+y^{\prime}+s+y}{2}, \xi\right)} & \leq e^{m L \omega\left(\frac{x+y^{\prime}+s+y}{2}\right)} e^{m L \omega(\xi)} e^{m L} \\
& \leq e^{m L \omega\left(2 \max \left\{|x|,\left|y^{\prime}\right|,|s|,|y|\right\}\right)} e^{m L \omega(\xi)} e^{m L} \\
& \leq e^{m L^{2} \omega(x)} e^{m L^{2} \omega\left(y^{\prime}\right)} e^{m L^{2} \omega(s)} e^{m L^{2} \omega(y)} e^{m L \omega(\xi)} e^{m L^{2}+m L} .
\end{aligned}
$$

By $(0.11)$ we deduce (since $\varphi^{*}(x) / x$ is increasing)

$$
e^{4(M+1) L^{2} \rho \varphi^{*}\left(\frac{\left|\delta_{1}+\tau_{1}+\sigma_{5}\right|}{4(M+1) L^{2}}\right)} \leq e^{M L^{2} \varphi^{*}\left(\frac{\left|\delta_{1}\right|}{M L^{2}}\right)} e^{M L^{2} \varphi^{*}\left(\frac{\left|\tau_{1}\right|}{M L^{2}}\right)} e^{(M+1) L^{2} \varphi^{*}\left(\frac{\left|\sigma_{5}\right|}{(M+1) L^{2}}\right)} .
$$

Then, as $\left|\sigma_{1}+\cdots+\sigma_{5}\right|=2\left(k-j^{\prime}\right) \leq 2 k$,

$$
e^{(M+1) L^{2} \varphi^{*}\left(\frac{\left|\sigma_{1}\right|}{(M+1) L^{2}}\right)} \cdots e^{(M+1) L^{2} \varphi^{*}\left(\frac{\left|\sigma_{5}\right|}{(M+1) L^{2}}\right)} \leq e^{(M+1) L^{2} \varphi^{*}\left(\frac{2 k}{(M+1) L^{2}}\right)} .
$$


Since $\psi \in \mathcal{S}_{\omega}\left(\mathbb{R}^{d}\right)$, for all $\mu>0$ there exists $C_{M, \mu}>0$ such that

$$
\left|D_{x}^{\delta_{2}} \overline{\psi(x)}\right|\left|D_{s}^{\tau_{2}} \psi(s)\right| \leq C_{M, \mu} e^{M L^{2} \varphi^{*}\left(\frac{\left|\delta_{2}\right|}{M L^{2}}\right)} e^{-\mu \omega(x)} e^{M L^{2} \varphi^{*}\left(\frac{\left|\tau_{2}\right|}{M L^{2}}\right)} e^{-\mu \omega(s)} .
$$

Therefore, by Lemma 0.8,

$$
\begin{aligned}
& \sum_{\substack{\delta_{1}+\delta_{2}=\delta \\
\tau_{1}+\tau_{2}=\tau}} \frac{\delta !}{\delta_{1} ! \delta_{2} !} \frac{\tau !}{\tau_{1} ! \tau_{2} !} e^{M L^{2} \varphi^{*}\left(\frac{|\delta 1|}{M L^{2}}\right)} e^{M L^{2} \varphi^{*}\left(\frac{\left|\delta_{2}\right|}{M L^{2}}\right)} e^{M L^{2} \varphi^{*}\left(\frac{\left|\tau_{1}\right|}{M L^{2}}\right)} e^{M L^{2} \varphi^{*}\left(\frac{\left|\tau_{2}\right|}{M L^{2}}\right)} \\
& \leq 2^{|\delta+\tau|} e^{M L^{2} \varphi^{*}\left(\frac{|\delta|}{M L^{2}}\right)} e^{M L^{2} \varphi^{*}\left(\frac{|\tau|}{M L^{2}}\right)} \\
& \leq e^{M L \varphi^{*}\left(\frac{|\delta|}{M L}\right)} e^{M L \varphi^{*}\left(\frac{|\tau|}{M L}\right)} e^{2 M L^{2}}
\end{aligned}
$$

On the other hand, by Lemma 0.1 ,

$$
\begin{aligned}
& \sum_{j^{\prime}=0}^{k}\left(\begin{array}{l}
k \\
j^{\prime}
\end{array}\right)_{\left|\sigma_{1}+\cdots+\sigma_{5}\right|=2\left(k-j^{\prime}\right)} \frac{\left(2\left(k-j^{\prime}\right)\right) !}{\sigma_{1} ! \cdots \sigma_{5} !} \\
& \quad=\sum_{j^{\prime}=0}^{k}\left(\begin{array}{l}
k \\
j^{\prime}
\end{array}\right) 5^{2\left(k-j^{\prime}\right)}=5^{2 k}\left(1+\frac{1}{5^{2}}\right)^{k}=(26)^{k}<\left(e^{2}\right)^{2 k}
\end{aligned}
$$

so by $(0.10)$,

$$
\left(e^{2}\right)^{2 k} e^{(M+1) L^{2} \varphi^{*}\left(\frac{2 k}{(M+1) L^{2}}\right)} \leq e^{(M+1) L^{2}+(M+1) L} e^{(M+1) \varphi^{*}\left(\frac{2 k}{M+1}\right)} .
$$

For any $\ell, h \in \mathbb{N}$, take $M \in \mathbb{N}$ such that

$$
M \geq C_{4} \max \{\ell, h\}
$$

Then, the series

$$
\sum_{\delta, \tau \in \mathbb{N}_{0}^{d}} e^{M L \varphi^{*}\left(\frac{|\delta|}{M L}\right)-h C_{4} \varphi^{*}\left(\frac{|\delta|}{h C_{4}}\right)} e^{M L \varphi^{*}\left(\frac{|\tau|}{M L}\right)-\ell C_{4} \varphi^{*}\left(\frac{|\tau|}{\ell C_{4}}\right)}
$$

converges (see (1.14)). Thus, for all $\mu>0$, there exists $C_{M, \mu}^{\prime}>0$ such that (for $M \geq C_{4} \max \{\ell, h\}$ )

$$
\begin{aligned}
\left|\lambda_{\ell, h, k}\right| \leq & C_{M, \mu}^{\prime} e^{(M+1) \varphi^{*}\left(\frac{2 k}{M+1}\right)}\left(C_{1} e^{C_{4}}\right)^{\ell+h} e^{-\ell C_{3} \omega(\xi-\eta)} e^{-h C_{3} \omega\left(\eta^{\prime}-\xi\right)} \times \\
& \times e^{\left((M+1) L^{2}+m L^{2}-\mu\right)(\omega(x)+\omega(s))} e^{m L^{2} \omega\left(y^{\prime}\right)} e^{m L^{2} \omega(y)} e^{m L \omega(\xi)}
\end{aligned}
$$

We choose

$$
\mu>(M+1) L^{2}+m L^{2}
$$


Therefore $\left|\lambda_{\ell, h, k}\right|$ is estimated by a function in $L^{1}\left(\mathbb{R}_{s, x}^{2 d}\right)$ for all $k \in \mathbb{N}_{0}$. Furthermore, since by $(0.1)$,

$$
e^{-\ell C_{3} \omega(\xi-\eta)} e^{-h C_{3} \omega\left(\eta^{\prime}-\xi\right)} \leq e^{-\ell C_{3} / L \omega(\eta)} e^{\left(\ell C_{3}-h C_{3} / L\right) \omega(\xi)} e^{h C_{3} \omega\left(\eta^{\prime}\right)} e^{(\ell+h) C_{3}},
$$

given $\ell \in \mathbb{N}$, we take

$$
h>\ell L+m L^{2} / C_{3} .
$$

Hence, the estimate of $\left|\lambda_{\ell, h, k}\right|$ in $(4.22)$ is in $L^{1}\left(\mathbb{R}_{s, x, \xi}^{3 d}\right)$, and therefore we can integrate by parts in $d \xi$.

From (4.23), again by (0.1) we have

$$
e^{-\ell C_{3} / L \omega(\eta)} e^{h C_{3} \omega\left(\eta^{\prime}\right)} \leq e^{-\ell C_{3} / L^{2} \omega\left(\eta-\eta^{\prime}\right)} e^{\left(\ell C_{3} / L+h C_{3}\right) \omega\left(\eta^{\prime}\right)} e^{\ell C_{3} / L} .
$$

Then, from (4.21), there exists $C_{M, \ell, h, \mu}>0$ such that, by the estimates (4.22) and (4.23),

$$
\begin{aligned}
\left|K\left(y^{\prime}, \eta^{\prime}, y, \eta\right)\right| \leq & C_{M, \ell, h, \mu}\left\langle y-y^{\prime}\right\rangle^{-2 k} e^{(M+1) \varphi^{*}\left(\frac{2 k}{M+1}\right)} \times \\
& \times e^{m L^{2}\left(\omega(y)+\omega\left(y^{\prime}\right)\right)} e^{-\ell C_{3} / L^{2} \omega\left(\eta-\eta^{\prime}\right)} e^{\left(\ell C_{3} / L+h C_{3}\right) \omega\left(\eta^{\prime}\right)} \times \\
& \times \int_{\mathbb{R}^{3 d}} e^{\left((M+1) L^{2}+m L^{2}-\mu\right)(\omega(x)+\omega(s))} e^{\left(m L+\ell C_{3}-h C_{3} / L\right) \omega(\xi)} d s d x d \xi
\end{aligned}
$$

for all $k \in \mathbb{N}_{0}$. Now, we take the infimum on $k$, and by (0.9) we obtain, for some $C_{M, \ell, h, \mu}^{\prime}>0$, that $\left|K\left(y^{\prime}, \eta^{\prime}, y, \eta\right)\right|$ is less than or equal to

$$
\begin{gathered}
C_{M, \ell, h, \mu}^{\prime} e^{-M \omega\left(\left\langle y-y^{\prime}\right\rangle\right)} e^{m L^{2}\left(\omega(y)+\omega\left(y^{\prime}\right)\right)} e^{-\ell C_{3} / L^{2} \omega\left(\eta-\eta^{\prime}\right)} e^{\left(\ell C_{3} / L+h C_{3}\right) \omega\left(\eta^{\prime}\right)} \times \\
\times \int_{\mathbb{R}^{3 d}} e^{\left((M+1) L^{2}+m L^{2}-\mu\right)(\omega(x)+\omega(s))} e^{\left(m L+\ell C_{3}-h C_{3} / L\right) \omega(\xi)} d s d x d \xi .
\end{gathered}
$$

Given $\ell \in \mathbb{N}$, by the same selection as before $\left(h>\ell L+m L^{2} / C_{3}\right.$ and $\mu>$ $\left.(M+1) L^{3}+m L^{2}\right)$, the integrals are convergent. Therefore, for every $\lambda>0$ there exist $C_{\lambda}, \mu_{\lambda}>0$ such that

$$
\left|K\left(y^{\prime}, \eta^{\prime}, y, \eta\right)\right| \leq C_{\lambda} e^{-\lambda \omega\left(y-y^{\prime}\right)} e^{-\lambda \omega\left(\eta-\eta^{\prime}\right)} e^{\mu_{\lambda} \omega\left(\eta^{\prime}\right)} e^{m L^{2}\left(\omega(y)+\omega\left(y^{\prime}\right)\right)}
$$

for all $\left(y^{\prime}, \eta^{\prime}, y, \eta\right) \in \mathbb{R}^{4 d}$. This shows formula (4.18). Notice that if in (4.24), we additionally take $M \geq C_{4} \max \{\ell, h\}$ satisfying $M \geq \ell+m L^{3}$, then by (0.1),

$$
e^{-M \omega\left(\left\langle y-y^{\prime}\right\rangle\right)} \leq e^{-\ell \omega\left(y-y^{\prime}\right)} e^{-m L^{2} \omega(y)+m L^{3} \omega\left(y^{\prime}\right)+m L^{3}} .
$$

Hence, for all $\lambda>0$, there exist $C_{\lambda}, \mu_{\lambda}>0$ such that

$$
\left|K\left(y^{\prime}, \eta^{\prime}, y, \eta\right)\right| \leq C_{\lambda} e^{-\lambda \omega\left(y-y^{\prime}\right)} e^{-\lambda \omega\left(\eta-\eta^{\prime}\right)} e^{\mu_{\lambda} \omega\left(\eta^{\prime}\right)} e^{\left(m L^{2}+m L^{3}\right) \omega\left(y^{\prime}\right)} .
$$


For the second part, we follow closely the proof of [61, Proposition 3.7]. By (4.22), taking the infimum on $k \in \mathbb{N}_{0}$ and using (0.9) and (0.1), we find $C_{M, \ell, h, k}^{\prime \prime}>0$ such that $\left|K\left(y^{\prime}, \eta^{\prime}, y, \eta\right)\right|$ is estimated by

$$
\begin{aligned}
& C_{M, \ell, h, \mu}^{\prime \prime} e^{-M \omega\left(\left\langle y-y^{\prime}\right\rangle\right)} e^{m L^{3} \omega\left(y-y^{\prime}\right)} e^{\left(m L^{2}+m L^{3}\right) \omega\left(y^{\prime}\right)} \times \\
& \quad \times \int_{\mathbb{R}^{3 d}} e^{-\ell C_{3} \omega(\xi-\eta)} e^{-h C_{3} \omega\left(\eta^{\prime}-\xi\right)} e^{\left((M+1) L^{2}+m L^{2}-\mu\right)(\omega(x)+\omega(s))} e^{m L \omega(\xi)} d s d x d \xi
\end{aligned}
$$

for all $\left(y^{\prime}, \eta^{\prime}, y, \eta\right) \in \mathbb{R}^{4 d}$. Now, assume $b(z)=0, z \in \Gamma \backslash \overline{B(0, R)}$. We set

$$
D_{y^{\prime}, y}:=\left\{(x, s, \xi) \in \mathbb{R}^{3 d}:\left(\frac{x+y^{\prime}+s+y}{2}, \xi\right) \in\left(\mathbb{R}^{2 d} \backslash \Gamma\right) \cup \overline{B(0, R)}\right\} .
$$

Let $\Gamma^{\prime}$ be an open conic subset of $\Gamma$ such that $\overline{\Gamma^{\prime} \cap S_{2 d-1}} \subseteq \Gamma$. We want to estimate $\left|K\left(y^{\prime}, \eta^{\prime}, y, \eta\right)\right|$ for all $\left(y^{\prime}, \eta^{\prime}\right) \in \Gamma^{\prime},(y, \eta) \in \mathbb{R}^{2 d}$. Similarly as in [61, (3.19)], there exists $\varepsilon>0$ such that

$$
\left|\frac{\left(y^{\prime}, \eta^{\prime}\right)}{\left|\left(y^{\prime}, \eta^{\prime}\right)\right|}-\frac{\left(\frac{x+y^{\prime}+s+y}{2}, \xi\right)}{\left|\left(y^{\prime}, \eta^{\prime}\right)\right|}\right| \geq \varepsilon
$$

for all $\left(y^{\prime}, \eta^{\prime}\right) \in \Gamma^{\prime},\left|\left(y^{\prime}, \eta^{\prime}\right)\right| \geq 2 R,(x, s, \xi) \in D_{y^{\prime}, y},(y, \eta) \in \mathbb{R}^{2 d}$. Then, by $(0.3)$ and $(0.1)$,

$$
\begin{aligned}
e^{\omega\left(\varepsilon\left(y^{\prime}, \eta^{\prime}\right)\right)} & \leq e^{\omega\left(\frac{y^{\prime}-x-s-y}{2}, \eta^{\prime}-\xi\right)} \\
& \leq e^{\omega\left(\max \left\{\left|y-y^{\prime}\right|,|x+s|\right\}, \eta^{\prime}-\xi\right)} \\
& \leq e^{L \omega\left(\max \left\{\left|y-y^{\prime}\right|,|x+s|\right\}\right)} e^{L \omega\left(\eta^{\prime}-\xi\right)} e^{L} \\
& \leq e^{L \omega\left(y-y^{\prime}\right)+L^{2} \omega(x)+L^{2} \omega(s)} e^{L \omega\left(\eta^{\prime}-\xi\right)} e^{L^{2}+L}
\end{aligned}
$$

Thus, for that $\varepsilon>0$, there exist $C_{\varepsilon}, L_{\varepsilon}>0$ such that

$$
e^{\frac{1}{2} \omega\left(y^{\prime}\right)} e^{\frac{1}{2} \omega\left(\eta^{\prime}\right)} \leq e^{\omega\left(y^{\prime}, \eta^{\prime}\right)} \leq C_{\varepsilon} e^{L_{\varepsilon}\left(\omega\left(y-y^{\prime}\right)+\omega(x)+\omega(s)+\omega\left(\eta^{\prime}-\xi\right)\right)} .
$$

Hence there are $C_{\varepsilon}^{\prime}, L_{\varepsilon}^{\prime}>0$ such that

$$
e^{-\omega\left(\eta^{\prime}-\xi\right)} \leq C_{\varepsilon}^{\prime} e^{-L_{\varepsilon}^{\prime} \omega\left(y^{\prime}\right)-L_{\varepsilon}^{\prime} \omega\left(\eta^{\prime}\right)} e^{\omega\left(y-y^{\prime}\right)+\omega(x)+\omega(s)} .
$$

Now, we put

$$
h=H h^{\prime}=(H-1) h^{\prime}+h^{\prime},
$$


for some $H>1$ and $h^{\prime}>0$ to be determined later. Therefore, by (4.27) and (0.1),

$$
\begin{aligned}
e^{-h C_{3} \omega\left(\eta^{\prime}-\xi\right)}= & e^{-(H-1) h^{\prime} C_{3} \omega\left(\eta^{\prime}-\xi\right)} e^{-h^{\prime} C_{3} \omega\left(\eta^{\prime}-\xi\right)} \\
\leq & \left(C_{\varepsilon}^{\prime}\right)^{(H-1) h^{\prime} C_{3}} e^{-(H-1) h^{\prime} C_{3} L_{\varepsilon}^{\prime} \omega\left(y^{\prime}\right)} e^{-(H-1) h^{\prime} C_{3} L_{\varepsilon}^{\prime} \omega\left(\eta^{\prime}\right)} \times \\
& \times e^{(H-1) h^{\prime} C_{3}\left(\omega\left(y-y^{\prime}\right)+\omega(x)+\omega(s)\right)} e^{-\left(h^{\prime} C_{3} / L\right) \omega(\xi)} e^{h^{\prime} C_{3} \omega\left(\eta^{\prime}\right)} e^{h^{\prime} C_{3}} .
\end{aligned}
$$

Again by (0.1),

$$
\begin{aligned}
e^{-\ell C_{3} \omega(\xi-\eta)} & \leq e^{-\left(\ell C_{3} / L\right) \omega\left(\eta-\eta^{\prime}\right)} e^{\ell C_{3} \omega\left(\eta^{\prime}-\xi\right)} e^{\ell C_{3}} \\
& \leq e^{-\left(\ell C_{3} / L\right) \omega\left(\eta-\eta^{\prime}\right)} e^{\ell C_{3} L \omega\left(\eta^{\prime}\right)} e^{\ell C_{3} L \omega(\xi)} e^{\ell C_{3}+\ell C_{3} L} .
\end{aligned}
$$

Hence, by (4.26) there exists $C_{M, \ell, h, \mu}^{\prime \prime \prime}>0$ such that $\left|K\left(y^{\prime}, \eta^{\prime}, y, \eta\right)\right|$ is less than or equal to

$$
\begin{aligned}
& C_{M, \ell, h, \mu}^{\prime \prime \prime} e^{-M \omega\left(\left\langle y-y^{\prime}\right\rangle\right)} e^{\left((H-1) h^{\prime} C_{3}+m L^{3}\right) \omega\left(y-y^{\prime}\right)} e^{\left(-(H-1) h^{\prime} C_{3} L_{\varepsilon}^{\prime}+m L^{2}+m L^{3}\right) \omega\left(y^{\prime}\right)} \times \\
& \quad \times e^{-\left(\ell C_{3} / L\right) \omega\left(\eta-\eta^{\prime}\right)} e^{\left(-(H-1) h^{\prime} C_{3} L_{\varepsilon}^{\prime}+h^{\prime} C_{3}+\ell C_{3} L\right) \omega\left(\eta^{\prime}\right)} \times \\
& \quad \times \int_{\mathbb{R}^{3 d}} e^{\left((M+1) L^{2}+m L^{2}+(H-1) h^{\prime} C_{3}-\mu\right)(\omega(x)+\omega(s))} e^{\left(-h^{\prime} C_{3} / L+\ell C_{3} L+m L\right) \omega(\xi)} d s d x d \xi .
\end{aligned}
$$

Given $\ell \in \mathbb{N}$ arbitrary, we denote $\lambda=\ell C_{3} / L>0$. We take $h^{\prime}>0$ such that

$$
\left(-h^{\prime} C_{3} / L+\ell C_{3} L+m L\right) \omega(\xi) \leq-\omega(\xi),
$$

and then $H>1$ with

$$
\begin{aligned}
& \left(-(H-1) h^{\prime} C_{3} L_{\varepsilon}^{\prime}+m L^{2}+m L^{3}\right) \omega\left(y^{\prime}\right) \leq-2 \lambda \omega\left(y^{\prime}\right) ; \\
& \left(-(H-1) h^{\prime} C_{3} L_{\varepsilon}^{\prime}+h^{\prime} C_{3}+\ell C_{3} L\right) \omega\left(\eta^{\prime}\right) \leq-2 \lambda \omega\left(\eta^{\prime}\right) .
\end{aligned}
$$

Now, for $M \in \mathbb{N}$ (which satisfies $M \geq C_{4} \max \left\{\ell, H h^{\prime}\right\}$ ) satisfying

$$
-M \omega\left(\left\langle y-y^{\prime}\right\rangle\right)+\left((H-1) h^{\prime} C_{3}+m L^{3}\right) \omega\left(y-y^{\prime}\right) \leq-\lambda \omega\left(y-y^{\prime}\right),
$$

and finally for $\mu>0$ large enough so that

$$
\left((M+1) L^{2}+m L^{2}+(H-1) h^{\prime} C_{3}-\mu\right)(\omega(x)+\omega(s)) \leq-(\omega(x)+\omega(s)),
$$

then the integrals are convergent by property $(\gamma)$ of the weight function, and hence (4.19) is satisfied for all $\left(y^{\prime}, \eta^{\prime}\right) \in \Gamma^{\prime},\left|\left(y^{\prime}, \eta^{\prime}\right)\right| \geq 2 R$ and $(y, \eta) \in \mathbb{R}^{2 d}$. The proof for $\left|\left(y^{\prime}, \eta^{\prime}\right)\right| \leq 2 R$ is immediate by (4.25). This completes the proof.

We now show, as in [14, Corollary 4.9], the corresponding extension of (4.11) for ultradistributions. 
Corollary 4.8. Let $b \in \mathrm{GS}_{\rho}^{m, \omega}, \psi \in \mathcal{S}_{\omega}\left(\mathbb{R}^{d}\right)$ with $\|\psi\|_{L^{2}\left(\mathbb{R}^{d}\right)}=1$, and $K$ as in (4.12). If $u \in \mathcal{S}_{\omega}^{\prime}\left(\mathbb{R}^{d}\right)$, then

$$
V_{\psi}\left(b^{w}(x, D) u\right)\left(y^{\prime}, \eta^{\prime}\right)=\int_{\mathbb{R}^{2 d}} K\left(y^{\prime}, \eta^{\prime}, y, \eta\right) V_{\psi} u(y, \eta) d y d \eta, \quad\left(y, \eta^{\prime}\right) \in \mathbb{R}^{2 d} .
$$

Proof. Since $V_{\psi}$ operates on $\mathcal{S}_{\omega}^{\prime}\left(\mathbb{R}^{d}\right)$, by Lemma 2.8 and Proposition 1.18, $V_{\psi} b^{w}(x, D)$ can be extended continuously to $\mathcal{S}_{\omega}^{\prime}\left(\mathbb{R}^{d}\right)$. Since $\mathcal{S}_{\omega}\left(\mathbb{R}^{d}\right)$ is dense in $\mathcal{S}_{\omega}^{\prime}\left(\mathbb{R}^{d}\right)$, for $u \in \mathcal{S}_{\omega}^{\prime}\left(\mathbb{R}^{d}\right)$ we can take a sequence $\left\{u_{n}\right\}$ in $\mathcal{S}_{\omega}\left(\mathbb{R}^{d}\right)$ such that $u_{n} \rightarrow$ $u$ in the topology of $\mathcal{S}_{\omega}^{\prime}\left(\mathbb{R}^{d}\right)$ (see for example [30, Lemma 14.7, Page 189]). By (4.11) and by the continuity of $V_{\psi} b^{w}: \mathcal{S}_{\omega}^{\prime}\left(\mathbb{R}^{d}\right) \rightarrow \mathcal{S}_{\omega}^{\prime}\left(\mathbb{R}^{2 d}\right)$ (Lemma 0.26),

$$
\int_{\mathbb{R}^{2 d}} K\left(y^{\prime}, \eta^{\prime}, y, \eta\right) V_{\psi} u_{n}(y, \eta) d y d \eta \rightarrow V_{\psi}\left(b^{w}(x, D) u\right)\left(y^{\prime}, \eta^{\prime}\right),
$$

in the topology of $\mathcal{S}_{\omega}^{\prime}\left(\mathbb{R}^{2 d}\right)$.

We claim

$$
\int_{\mathbb{R}^{2 d}} K\left(y^{\prime}, \eta^{\prime}, y, \eta\right) V_{\psi} u_{n}(y, \eta) d y d \eta \rightarrow \int_{\mathbb{R}^{2 d}} K\left(y^{\prime}, \eta^{\prime}, y, \eta\right) V_{\psi} u(y, \eta) d y d \eta
$$

in $\mathcal{S}_{\omega}^{\prime}\left(\mathbb{R}^{2 d}\right)$. First, it follows from [39, Theorem 2.4] that $V_{\psi} u_{n}(y, \eta)$ converges pointwise to $V_{\psi} u(y, \eta)$ for all $(y, \eta) \in \mathbb{R}^{2 d}$. As $\left\{u_{n}\right\}$ is bounded in $\mathcal{S}_{\omega}^{\prime}\left(\mathbb{R}^{d}\right)$, we have that $\left\{u_{n}\right\}_{n \in \mathbb{N}}$ is equicontinuous in $\mathcal{S}_{\omega}^{\prime}\left(\mathbb{R}^{d}\right)$. Then, there exists $C>0$ and a seminorm $q$ on $\mathcal{S}_{\omega}\left(\mathbb{R}^{d}\right)$ such that

$$
\left|\left\langle u_{n}, f\right\rangle\right| \leq C q(f), \quad f \in \mathcal{S}_{\omega}\left(\mathbb{R}^{d}\right) .
$$

Hence, from [39, Theorem 2.4], by (0.33) there exist $\tilde{C}, \tilde{\lambda}>0$ independent of $z \in \mathbb{R}^{2 d}$ and $n \in \mathbb{N}$ such that (fixing $f \in \mathcal{S}_{\omega}\left(\mathbb{R}^{d}\right)$ )

$$
\left|V_{\psi} u_{n}(z)\right| \leq \tilde{C} e^{\tilde{\lambda} \omega(z)} .
$$

Therefore, by (4.18), we have that for all $\lambda>0$ there exist $C_{\lambda}, \mu_{\lambda}>0$ such that

$$
\begin{aligned}
& \left|K\left(y^{\prime}, \eta^{\prime}, y, \eta\right)\right|\left|V_{\psi} u_{n}(y, \eta)\right| \\
& \quad \leq C_{\lambda} e^{-\lambda L \omega\left(y-y^{\prime}\right)} e^{-\lambda L \omega\left(\eta-\eta^{\prime}\right)} e^{\mu_{\lambda} \omega\left(\eta^{\prime}\right)} e^{\max \left\{0, m L^{2}\right\}\left(\omega(y)+\omega\left(y^{\prime}\right)\right)} \tilde{C} e^{\tilde{\lambda} \omega(y, \eta)} .
\end{aligned}
$$

By (0.1) and (0.3), there exists $C_{\lambda}^{\prime}>0$ such that, for each $n \in \mathbb{N}$,

$$
\begin{aligned}
& \left|K\left(y^{\prime}, \eta^{\prime}, y, \eta\right)\right|\left|V_{\psi} u_{n}(y, \eta)\right| \\
& \quad \leq C_{\lambda}^{\prime} e^{\left(-\lambda+\max \left\{0, m L^{2}\right\}+\tilde{\lambda} L\right) \omega(y)} e^{(-\lambda+\tilde{\lambda} L) \omega(\eta)} e^{\left(\lambda L+\max \left\{0, m L^{2}\right\}\right) \omega\left(y^{\prime}\right)} e^{\left(\lambda L+\mu_{\lambda}\right) \omega\left(\eta^{\prime}\right)} .
\end{aligned}
$$


Taking $\lambda>\max \left\{0, m L^{2}\right\}+\tilde{\lambda} L$, we have that $\left|K\left(y^{\prime}, \eta^{\prime}, y, \eta\right)\right|\left|V_{\psi} u_{n}(y, \eta)\right|$ is dominated by a function in $L^{1}\left(\mathbb{R}_{y, \eta}^{2 d}\right)$ and therefore by the Lebesgue theorem we obtain (4.28) pointwise. This clearly implies, again by Lebesgue theorem, the convergence in $(4.28)$ in $\mathcal{S}_{\omega}^{\prime}\left(\mathbb{R}^{2 d}\right)$. By the uniqueness of the limit the result follows.

We prove [14, Proposition 4.11] for the Weyl quantization. In that result, $\omega$ was assumed to be subadditive.

Proposition 4.9. Let $\omega$ be a weight function and $b \in \mathrm{GS}_{\rho}^{m, \omega}$ for some $m \in \mathbb{R}$, $0<\rho \leq 1$. Then,

$$
\mathrm{WF}_{\omega}^{\prime}\left(b^{w}(x, D) u\right) \subset \text { conesupp }(b), \quad u \in \mathcal{S}_{\omega}^{\prime}\left(\mathbb{R}^{d}\right) .
$$

Proof. Let $\psi \in \mathcal{S}_{\omega}\left(\mathbb{R}^{d}\right)$ with $\|\psi\|_{L^{2}\left(\mathbb{R}^{d}\right)}=1$ and let $0 \neq z_{0} \notin \operatorname{conesupp}(b)$. Then, there exists an open conic set $\Gamma \subseteq \mathbb{R}^{2 d} \backslash\{0\}, z_{0} \in \Gamma$, such that $b(z)=0$ for all $z \in \Gamma \backslash \overline{B(0, R)}$ for some $R>0$. Thus, from Theorem 4.7, for all open conic set $\Gamma^{\prime} \subseteq \mathbb{R}^{2 d} \backslash\{0\}$ such that $\overline{\Gamma^{\prime} \cap S_{2 d-1}} \subseteq \Gamma$, we have that $K\left(y^{\prime}, \eta^{\prime}, y, \eta\right)$ as in (4.12) satisfies (4.19) for all $\left(y^{\prime}, \eta^{\prime}\right) \in \Gamma^{\prime}$ and $(y, \eta) \in \mathbb{R}^{2 d}$. Moreover, by Lemma 0.26 there are $c, \mu>0$ such that for all $\lambda>0$ there exists $C_{\lambda}>0$ with

$$
\begin{aligned}
\left|V_{\psi}\left(b^{w}(x, D) u\right)\left(y^{\prime}, \eta^{\prime}\right)\right| \leq & \int_{\mathbb{R}^{2 d}}\left|K\left(y^{\prime}, \eta^{\prime}, y, \eta\right)\right|\left|V_{\psi} u(y, \eta)\right| d y d \eta \\
\leq & \int_{\mathbb{R}^{2 d}} C_{\lambda} e^{-(\lambda+\mu L) L \omega\left(y-y^{\prime}\right)} e^{-(\lambda+\mu L) L \omega\left(\eta-\eta^{\prime}\right)} \times \\
& \times e^{-2(\lambda+\mu L) L \omega\left(y^{\prime}\right)} e^{-2(\lambda+\mu L) L \omega\left(\eta^{\prime}\right)} c e^{\mu \omega(y, \eta)} d y d \eta
\end{aligned}
$$

for all $\left(y^{\prime}, \eta^{\prime}\right) \in \Gamma^{\prime}$. From (0.1), it follows

$$
e^{-(\lambda+\mu L) L \omega\left(y-y^{\prime}\right)} e^{-2(\lambda+\mu L) L \omega\left(y^{\prime}\right)} \leq e^{-(\lambda+\mu L) \omega(y)} e^{-\lambda L \omega\left(y^{\prime}\right)} e^{(\lambda+\mu L) L},
$$

and respectively for $\eta, \eta^{\prime}$. From (0.3),

$$
e^{\mu \omega(y, \eta)} \leq e^{\mu L \omega(y)} e^{\mu L \omega(\eta)} e^{\mu L} .
$$

Therefore, again by (0.3),

$$
\begin{aligned}
& \left|V_{\psi}\left(b^{w}(x, D) u\right)\left(y^{\prime}, \eta^{\prime}\right)\right| \\
& \quad \leq c C_{\lambda} e^{2(\lambda+\mu L) L} e^{\mu L} e^{-\lambda L \omega\left(y^{\prime}\right)} e^{-\lambda L \omega\left(\eta^{\prime}\right)} \int_{\mathbb{R}^{2 d}} e^{-\lambda \omega(y)} e^{-\lambda \omega(\eta)} d y d \eta \\
& \quad \leq c C_{\lambda} e^{2(\lambda+\mu L) L} e^{\mu L} e^{\lambda L} e^{-\lambda \omega\left(y^{\prime}, \eta^{\prime}\right)} \int_{\mathbb{R}^{2 d}} e^{-\lambda \omega(y)} e^{-\lambda \omega(\eta)} d y d \eta,
\end{aligned}
$$


for all $\left(y^{\prime}, \eta^{\prime}\right) \in \Gamma^{\prime}$. As the integral converges, we have that for all $\lambda>0$, there exists $C_{\lambda}^{\prime}>0$ such that

$$
\sup _{z \in \Gamma^{\prime}} e^{\lambda \omega(z)}\left|V_{\psi}\left(b^{w}(x, D) u\right)(z)\right| \leq C_{\lambda}^{\prime} .
$$

Hence, $z_{0} \notin \mathrm{WF}_{\omega}^{\prime}\left(b^{w}(x, D) u\right)$ by Definition 4.1 as we wanted.

Corollary 4.10. Let $b$ be a global symbol in $\mathrm{GS}_{\rho}^{m, \omega}$ with compact support. Then, its Weyl quantization $b^{w}(x, D)$ is $\omega$-regularizing (in the sense of Definition 1.20).

Proof. Since the support of $b$ is compact, it follows that conesupp $(b)=\emptyset$, hence by Proposition 4.9 we have $\mathrm{WF}_{\omega}^{\prime}\left(b^{w}(x, D) u\right)=\emptyset, u \in \mathcal{S}_{\omega}^{\prime}\left(\mathbb{R}^{d}\right)$. From $[14$, Proposition 3.18] we obtain that $b^{w}(x, D) u \in \mathcal{S}_{\omega}\left(\mathbb{R}^{d}\right)$ for all $u \in \mathcal{S}_{\omega}^{\prime}\left(\mathbb{R}^{d}\right)$.

\subsection{The Weyl wave front set}

In this section we introduce a new global wave front set given in terms of the Weyl quantization in the ultradifferentiable setting, similarly to the one introduced by Hörmander [45, Definition 2.1] in the classical setting. Some restrictions on the weight functions will be necessary, since the definition of wave front set is based on the construction of the parametrix of Chapter 3 .

Definition 4.11. Let $a \in \mathrm{GS}_{\rho}^{m, \omega}$. We say that $z_{0} \in \mathbb{R}^{2 d} \backslash\{0\}$ is noncharacteristic for $a$ if there exist a Gevrey weight function $\sigma$ with $\omega\left(t^{1 / \rho}\right)=$ $o(\sigma(t))$ as $t \rightarrow \infty, C_{1}, C_{2}>0, n \in \mathbb{N}, R \geq 1$, and an open conic set $\Gamma \subset \mathbb{R}^{2 d} \backslash\{0\}$ with $z_{0} \in \Gamma$ such that

$$
\begin{aligned}
|a(z)| & \geq C_{1} e^{m \omega(z)}, \quad \text { and } \\
\left|D^{\alpha} a(z)\right| & \leq C_{2}^{|\alpha|}\langle z\rangle^{-\rho|\alpha|} e^{\frac{1}{n} \varphi_{\sigma}^{*}(n|\alpha|)}|a(z)|,
\end{aligned}
$$

for all $\alpha \in \mathbb{N}_{0}^{2 d}$ and $z \in \Gamma,|z| \geq R$.

Given $a \in \mathrm{GS}_{\rho}^{m, \omega}$ we define the characteristic set of $a$, denoted by $\operatorname{char}(a)$, to be the complement in $\mathbb{R}^{2 d}$ of the set of non-characteristic points for $a$ in the sense of Definition 4.11. We have, for all $a \in \mathrm{GS}_{\rho}^{m, \omega}$,

$$
\operatorname{conesupp}(a) \cup \operatorname{char}(a)=\mathbb{R}^{2 d} \backslash\{0\} .
$$

In fact, we take $0 \neq z_{0} \notin \operatorname{conesupp}(a) \cup \operatorname{char}(a)$. Then, there exist open conic sets $\Gamma, \Gamma^{\prime} \subseteq \mathbb{R}^{2 d} \backslash\{0\}, z_{0} \in \Gamma \cap \Gamma^{\prime}$, such that $a(z)=0$ for all $z \in \Gamma,|z| \geq R$ for 
some $R>0$, and $|a(z)| \geq C_{1} e^{m \omega(z)}$ for all $z \in \Gamma^{\prime},|z| \geq R^{\prime}$ for some $C_{1}, R^{\prime}>0$. Therefore, there exists $\lambda>0$ large enough such that

$$
0=\left|a\left(\lambda z_{0}\right)\right| \geq C_{1} e^{m \omega\left(\lambda z_{0}\right)}>0,
$$

which is a contradiction.

Definition 4.12. Let $\omega$ be a weight function, $0<\rho \leq 1$ and $u \in \mathcal{S}_{\omega}^{\prime}\left(\mathbb{R}^{d}\right)$. We say that $z \in \mathbb{R}^{2 d} \backslash\{0\}$ is not in the Weyl wave front set $\mathrm{WF}_{\rho}^{\omega}(u)$ of $u$ if there exist $m \in \mathbb{R}$ and $a \in \mathrm{GS}_{\rho}^{m, \omega}$ such that $a^{w}(x, D) u \in \mathcal{S}_{\omega}\left(\mathbb{R}^{d}\right)$ and $z$ is non-characteristic for $a$.

We show that the global symbol in Definition 4.12, similarly as in [61, Proposition 2.7], can be taken without loss of generality of order zero. To this, we notice that there exist weight functions as in Definition 0.3 that cannot be dominated by any subadditive function that satisfies property $(\beta)([35])$. This motivates the following definition.

Definition 4.13. Fix $0<\rho \leq 1$. A weight function $\omega$ is called $\rho$-regular if for all $m \in \mathbb{R}$ there exists $a \in \mathrm{GS}_{\rho}^{m, \omega}$ such that for some Gevrey weight function $\sigma$ with $\omega\left(t^{1 / \rho}\right)=o(\sigma(t))$ as $t \rightarrow \infty$, the inequalities (4.29) and (4.30) hold for all $z \in \mathbb{R}^{2 d}$ with $|z| \geq R$, for some $R \geq 1$.

Example 4.14. The Gevrey weight functions $\omega(t)=t^{a}, 0<a<1 / 2$, are $(1-a)$-regular.

Proof. For $m \in \mathbb{R}$, let $p$ be as in (3.11). Clearly, $p$ satisfies (4.29) for all $z \in \mathbb{R}^{2 d}$. By (3.15), using Lemma $0.9\left(\right.$ as $\omega(t)=o\left(t^{1-a}\right)$ as $\left.t \rightarrow \infty\right)$ we have that $p \in \mathrm{GS}_{\rho}^{m, \omega}$ for $\rho=1-a$. Again by (3.15), using formula (0.12) for some Gevrey weight function $\sigma$ such that $\omega\left(t^{1 / \rho}\right)=o(\sigma(t))$ as $t \rightarrow \infty$, we find $C>0$ such that for all $\alpha \in \mathbb{N}_{0}^{2 d}, z \in \mathbb{R}^{2 d}$,

$$
\left|D^{\alpha} p(z)\right| \leq C^{|\alpha|} \alpha !\langle z\rangle^{-(1-a)|\alpha|}|p(z)| \leq C^{|\alpha|}\langle z\rangle^{-\rho|\alpha|} e^{\varphi_{\sigma}^{*}(|\alpha|)}|p(z)|,
$$

and this shows (4.30).

Example 4.15. The weight $\omega(t)=\log (1+t)$ is $\rho$-regular, for all $0<\rho \leq 1$.

Proof. Fix $0<\rho \leq 1$. For $m \in \mathbb{R}$, let $p(z):=\langle z\rangle^{m}, z \in \mathbb{R}^{2 d}$. It satisfies (4.29): we have $\log (1+|z|) \geq \log (\langle z\rangle) \geq \log (1+|z|)-1$ for all $z \in \mathbb{R}^{2 d}$. Thus,

$$
|p(z)|=e^{m \log (\langle z\rangle)} \geq e^{\min \{0,-m\}} e^{m \omega(z)} .
$$


Now, we write $p$ as the composition $p(z)=g(u(z)), z \in \mathbb{R}^{2 d}$, where

$$
\begin{aligned}
& g(t)=t^{m / 2}, \quad m \in \mathbb{R}, t \geq 1 \\
& u(z)=\langle z\rangle^{2}=1+z_{1}^{2}+\cdots+z_{2 d}^{2}, \quad z=\left(z_{1}, \ldots, z_{2 d}\right) \in \mathbb{R}^{2 d} .
\end{aligned}
$$

We want to use Faà di Bruno formula (3.12). First, we observe that

$$
\left|g^{(k)}(t)\right| \leq\left|\frac{m}{2} \| \frac{m}{2}-1\right| \cdots\left|\frac{m}{2}-k+1\right||t|^{m / 2-k},
$$

for all $k \in \mathbb{N}_{0}$. Therefore,

$$
\begin{aligned}
\left|g^{(k)}(u(z))\right| & \leq\left|\frac{m}{2}\right|\left|\frac{m}{2}-1\right| \cdots\left|\frac{m}{2}-k+1\right|\langle z\rangle^{m-2 k} \\
& =\left|\frac{m}{2}\right|\left|\frac{m}{2}-1\right| \cdots\left|\frac{m}{2}-k+1\right|\langle z\rangle^{-2 k} p(z) .
\end{aligned}
$$

On the other hand, the derivatives of $u$ are given in (3.13). Hence, using (3.14), we get, by (3.12),

$$
\begin{aligned}
\left|D^{\alpha} p(z)\right| \leq & \sum_{0 \leq k \leq|\alpha|}\left|\frac{m}{2}\right|\left|\frac{m}{2}-1\right| \cdots\left|\frac{m}{2}-k+1\right|\langle z\rangle^{-2 k} p(z) \alpha ! \times \\
& \times \sum_{*} 2^{|\alpha|}\langle z\rangle^{2 k-|\alpha|} \prod_{j=1}^{2 d} \frac{1}{c_{e_{j}} !} \frac{1}{c_{2 e_{j}} !}
\end{aligned}
$$

for all $\alpha \in \mathbb{N}_{0}^{2 d}, z \in \mathbb{R}^{2 d}$, where $\sum_{*}$ is the sum of all $c_{\beta} \in \mathbb{N}_{0}$ such that $\sum_{|\beta|>0} c_{\beta}=k$ and $\sum_{|\beta|>0} \beta c_{\beta}=\alpha$. Proceeding as in Section 3.2 there exists $C>0$ such that for all $\alpha \in \mathbb{N}_{0}^{2 d}, z \in \mathbb{R}^{2 d}$ we have

$$
\left|D^{\alpha} p(z)\right| \leq C^{|\alpha|} \alpha !\langle z\rangle^{-|\alpha|} p(z) \leq C^{|\alpha|} \alpha !\langle z\rangle^{-\rho|\alpha|} p(z) \text {. }
$$

Since $\omega(t)=\log (1+t)=o\left(t^{\rho}\right)$ as $t \rightarrow \infty$, we use Lemma 0.9 to obtain $p \in \mathrm{GS}_{\rho}^{m, \omega}$. By (0.12), choosing any Gevrey weight function $\sigma$ we obtain (4.30) since $\omega\left(t^{1 / \rho}\right)=o(\sigma(t))$ as $t \rightarrow \infty$.

We observe that, for $\omega(t)=\log (1+t)$, the class of symbols $\mathrm{GS}_{1}^{m, \omega}$ coincides with [61, Definition 2.2]. However, Definition 4.11 might not be [61, Definition 2.4 , as condition (4.30) is not required in the latter definition.

The following lemma is taken from [34, Lemma 4]. The weight function must satisfy property $(\beta)$. 
Lemma 4.16. Given a weight function $\sigma$ and two cones $\Gamma, \Gamma^{\prime} \subseteq \mathbb{R}^{2 d} \backslash\{0\}$ such that $\overline{\Gamma^{\prime} \cap S_{2 d-1}} \subseteq \Gamma$, there exists $\chi \in C^{\infty}\left(\mathbb{R}^{2 d}\right)$ such that $0 \leq \chi \leq 1$, $\operatorname{supp} \chi \subseteq \Gamma, \chi(z)=1$ for $z \in \Gamma^{\prime}$ with $|z| \geq 1$ and for every $k \in \mathbb{N}$ there is $C_{k}>0$ such that

$$
\left|D^{\alpha} \chi(z)\right| \leq C_{k}\langle z\rangle^{-|\alpha|} e^{k \varphi_{\sigma}^{*}\left(\frac{|\alpha|}{k}\right)}, \quad \alpha \in \mathbb{N}_{0}^{2 d}, z \in \mathbb{R}^{2 d} .
$$

Moreover, if $\omega$ satisfies $\omega\left(t^{1 / \rho}\right)=o(\sigma(t))$ as $t \rightarrow \infty$, for some $0<\rho \leq 1$, then $\chi \in \mathrm{GS}_{\rho}^{0, \omega}$.

Proof. To see the last assertion, it is enough to use Lemma 0.10(1).

Proposition 4.17. Let $\omega$ be a $\rho$-regular weight function, for some $0<\rho \leq 1$, $u \in \mathcal{S}_{\omega}^{\prime}\left(\mathbb{R}^{d}\right)$, and $0 \neq z_{0} \notin \mathrm{WF}_{\rho}^{\omega}(u)$. There exist $b \in \mathrm{GS}_{\rho}^{0, \omega}$ and an open conic set $\Gamma \subset \mathbb{R}^{2 d} \backslash\{0\}$ such that $z_{0} \in \Gamma, 0 \leq b \leq 1, b(z)=1$ for $z \in \Gamma$ with $|z| \geq 1$ and $b^{w}(x, D) u \in \mathcal{S}_{\omega}\left(\mathbb{R}^{d}\right)$.

Proof. Since $0 \neq z_{0} \notin \mathrm{WF}_{\rho}^{\omega}(u)$, there exist $m \in \mathbb{R}$ and $a \in \mathrm{GS}_{\rho}^{m, \omega}$ such that $a^{w}(x, D) u \in \mathcal{S}_{\omega}\left(\mathbb{R}^{d}\right)$, a Gevrey weight function $\sigma$ such that $\omega\left(t^{1 / \rho}\right)=o(\sigma(t))$ as $t \rightarrow \infty, C_{1}, C_{2}>0, n \in \mathbb{N}, R \geq 1$, and an open conic set $\Gamma \subset \mathbb{R}^{2 d} \backslash\{0\}$ such that $z_{0} \in \Gamma$, and $a$ satisfies (4.29) and (4.30) for all $z \in \Gamma,|z| \geq R$. We can take $C_{2} \geq 1$.

We have, by (3.5),

$(\bar{a} \# a)(x, \xi) \sim \sum_{j=0}^{\infty} a_{j}(x, \xi)=\sum_{j=0}^{\infty} \sum_{|\beta+\gamma|=j} \frac{(-1)^{|\beta|}}{\gamma ! \beta !} 2^{-|\beta+\gamma|} \partial_{\xi}^{\gamma} D_{x}^{\beta} \bar{a}(x, \xi) \partial_{\xi}^{\beta} D_{x}^{\gamma} a(x, \xi)$.

By Proposition 2.15, it follows that $\sum a_{j} \in \mathrm{FGS}_{\rho}^{2 m, \omega}$. We use formula (2.14) (with $N=1$ ) to obtain that, for some $\bar{C}>0$,

$$
|(\bar{a} \# a)(z)| \geq|a(z)|^{2}-\left|\left(a-a_{0}\right)(z)\right| \geq C_{1}^{2} e^{2 m \omega(z)}-\bar{C}\langle z\rangle^{-\rho} e^{2 m \omega(z)},
$$

for all $z \in \Gamma,|z| \geq R$. Thus, for $z \in \Gamma,\langle z\rangle \geq \max \left\{R,\left(2 \bar{C} / C_{1}^{2}\right)^{1 / \rho}\right\}$, we have

$$
|(\bar{a} \# a)(z)| \geq \frac{C_{1}^{2}}{2} e^{2 m \omega(z)} .
$$

On the other hand, by (2.12),

$$
(\bar{a} \# a)(x, \xi)=|a(x, \xi)|^{2}+\sum_{k=1}^{\infty} \sum_{j=j_{k}}^{j_{k+1}-1} \Psi_{j, k}(x, \xi) a_{j}(x, \xi),
$$


where $\left(\Psi_{j, k}\right)$ is defined in (2.4) (see the proof of Theorem 2.6 for the conditions on the sequence $\left.\left(j_{k}\right)\right)$. For all $j \in \mathbb{N}$, we have by Leibniz rule

$$
\begin{aligned}
& \left|D_{x}^{\alpha} D_{\xi}^{\epsilon} a_{j}(x, \xi)\right| \\
& \leq \sum_{|\beta+\gamma|=j} \frac{1}{\gamma ! \beta !} 2^{-|\beta+\gamma|} \sum_{\substack{\widetilde{\alpha} \leq \alpha \\
\tilde{\epsilon} \leq \epsilon}}\left(\begin{array}{c}
\alpha \\
\widetilde{\alpha}
\end{array}\right)\left(\begin{array}{c}
\epsilon \\
\widetilde{\epsilon}
\end{array}\right)\left|D_{x}^{\alpha-\widetilde{\alpha}+\beta} D_{\xi}^{\epsilon-\widetilde{\epsilon}+\gamma} \bar{a}(x, \xi)\right|\left|D_{x}^{\widetilde{\alpha}+\gamma} D_{\xi}^{\tilde{\epsilon}+\beta} a(x, \xi)\right| \\
& \leq \sum_{|\beta+\gamma|=j} \frac{1}{\gamma ! \beta !} 2^{-|\beta+\gamma|} \sum_{\substack{\widetilde{\alpha} \leq \alpha \\
\tilde{\epsilon} \leq \epsilon}}\left(\begin{array}{c}
\alpha \\
\widetilde{\alpha}
\end{array}\right)\left(\begin{array}{c}
\epsilon \\
\widetilde{\epsilon}
\end{array}\right) C_{2}^{|\alpha+\epsilon+2 \gamma+2 \beta|}\langle(x, \xi)\rangle^{-\rho|\alpha+\epsilon+2 \gamma+2 \beta|} \times \\
& \quad \times e^{\frac{1}{n} \varphi_{\sigma}^{*}(n|\alpha-\widetilde{\alpha}+\epsilon-\widetilde{\epsilon}+\beta+\gamma|)} e^{\frac{1}{n} \varphi_{\sigma}^{*}(n|\widetilde{\alpha}+\widetilde{\epsilon}+\beta+\gamma|)}|\bar{a}(x, \xi)||a(x, \xi)|,
\end{aligned}
$$

for all $\alpha, \epsilon \in \mathbb{N}_{0}^{d},(x, \xi) \in \Gamma,|(x, \xi)| \geq R$. Then, proceeding as in Example 2.12, the derivatives of $a_{j}$ are estimated, for all $j \in \mathbb{N}$, by

$$
e^{2 d^{2}}\left(2 C_{2}\right)^{|\alpha+\epsilon|}\left(\frac{C_{2}^{2}}{2}\right)^{j}\langle(x, \xi)\rangle^{-\rho(|\alpha+\epsilon|+2 j)} e^{\frac{1}{n} \varphi_{\sigma}^{*}(n(|\alpha+\epsilon|+2 j))}|\bar{a}(x, \xi)||a(x, \xi)|,
$$

for all $\alpha, \epsilon \in \mathbb{N}_{0}^{d},(x, \xi) \in \Gamma,|(x, \xi)| \geq R$. We have that, by (0.11) and Lemma $0.10(1)$, for all $k \in \mathbb{N}$ there exists $C_{k}>0$ such that

$$
e^{\frac{1}{n} \varphi_{\sigma}^{*}(n(|\alpha+\epsilon|+2 j))} \leq e^{\frac{1}{2 n} \varphi_{\sigma}^{*}(2 n|\alpha+\epsilon|)} e^{\frac{1}{2 n} \varphi_{\sigma}^{*}(2 n(2 j))} \leq e^{\frac{1}{2 n} \varphi_{\sigma}^{*}(2 n|\alpha+\epsilon|)} C_{k} e^{k \rho \varphi_{\omega}^{*}\left(\frac{2 j}{k}\right)} .
$$

Therefore, when estimating

$$
\left|D_{x}^{\alpha} D_{\xi}^{\epsilon}\left(a-a_{0}\right)(x, \xi)\right| \leq \sum_{k=1}^{\infty} \sum_{j=j_{k}}^{j_{k+1}-1}\left|D_{x}^{\alpha} D_{\xi}^{\epsilon}\left(\Psi_{j, k} a_{j}\right)(x, \xi)\right|
$$

we can obtain by the definition of $\Psi_{j, k}$ in (2.4) (see also (2.7)) and taking by induction the sequence $\left(j_{k}\right)$ as in the proof of Theorem 2.6 the following estimate: there exist $C^{\prime}>0$ and $C_{3}>0$ (which depends on $C_{2}>0$ ) such that for all $\alpha, \epsilon \in \mathbb{N}_{0}^{d}$ and $(x, \xi) \in \Gamma,|(x, \xi)| \geq R$,

$$
\left|D_{x}^{\alpha} D_{\xi}^{\epsilon}\left(a-a_{0}\right)(x, \xi)\right| \leq C^{\prime} C_{3}^{|\alpha+\epsilon|}\langle(x, \xi)\rangle^{-\rho|\alpha+\epsilon|} e^{\frac{1}{2 n} \varphi_{\sigma}^{*}(2 n|\alpha+\epsilon|)}|\bar{a}(x, \xi)||a(x, \xi)| .
$$

Hence, we can assume that $a \in \mathrm{GS}_{\rho}^{m, \omega}$ can be written as $a^{\prime}+a^{\prime \prime}$, where $a^{\prime} \in \mathrm{GS}_{\rho}^{m, \omega}, a^{\prime} \geq 0$ and satisfies, for probably another $R \geq 1$,

$$
a^{\prime}(z) \geq C_{1} e^{m \omega(z)}, \quad z \in \Gamma,|z| \geq R
$$

and (4.30), and $a^{\prime \prime}$ satisfies (see (4.31))

$$
\left|a^{\prime \prime}(z)\right| \leq \bar{C}\langle z\rangle^{-\rho} e^{m \omega(z)}, \quad z \in \Gamma,|z| \geq R
$$


Since $\omega$ is a $\rho$-regular weight function, there exist a global symbol $a_{0}$ in $\mathrm{GS}_{\rho}^{m, \omega}$ and a Gevrey weight function $\sigma^{\prime}$ such that formulas (4.29) and (4.30) are satisfied for $a_{0}$, for some $C_{1}^{\prime}, C_{2}^{\prime}>0, n^{\prime} \in \mathbb{N}$, for all $z \in \mathbb{R}^{2 d}$ with $|z| \geq R^{\prime}$, for some $R^{\prime} \geq 1$. Then, for the Gevrey weight function

$$
\min \left\{\sigma(t), \sigma^{\prime}(t)\right\}, \quad t \geq 1,
$$

the global symbols $a$ and $a_{0}$ in $\mathrm{GS}_{\rho}^{m, \omega}$ satisfy (4.29), (4.30) for $\min \left\{C_{1}, C_{1}^{\prime}\right\}>0$, $\max \left\{C_{2}, C_{2}^{\prime}\right\} \geq 1, \max \left\{n, n^{\prime}\right\} \in \mathbb{N}$, and $\max \left\{R, R^{\prime}\right\} \geq 1$. By abuse of notation, we denote this Gevrey weight function by $\sigma$, and the constants by $C_{1}, C_{2}>0$, $n \in \mathbb{N}$, and $R \geq 1$. Proceeding as before, we can decompose $a_{0}=a_{0}^{\prime}+a_{0}^{\prime \prime}$, where $a_{0}^{\prime} \in \mathrm{GS}_{\rho}^{m, \omega}, a_{0}^{\prime} \geq 0$, satisfying for $R \geq 1$ large enough,

$$
a_{0}^{\prime}(z) \geq C_{1} e^{m \omega(z)}, \quad|z| \geq R,
$$

and $a_{0}^{\prime \prime}$ satisfies, for some $C^{\prime}>0$,

$$
\left|a_{0}^{\prime \prime}(z)\right| \leq C^{\prime}\langle z\rangle^{-\rho} e^{m \omega(z)}, \quad|z| \geq R .
$$

Let $\Gamma^{\prime}, \Gamma^{\prime \prime} \subset \mathbb{R}^{2 d} \backslash\{0\}$ be open conic sets such that $z_{0} \in \Gamma^{\prime \prime}, \overline{\Gamma^{\prime \prime} \cap S_{2 d-1}} \subset \Gamma^{\prime}$ and $\overline{\Gamma^{\prime} \cap S_{2 d-1}} \subset \Gamma$. For the weight function $\sigma$, let $\chi$ and $b$ be as in Lemma 4.16 for $\Gamma, \Gamma^{\prime}$, and $\Gamma^{\prime}, \Gamma^{\prime \prime}$. Therefore $b \in \mathrm{GS}_{\rho}^{0, \omega}, 0 \leq b \leq 1, \operatorname{supp} b \subseteq \Gamma^{\prime}$, and $b(z)=1$ for $z \in \Gamma^{\prime \prime}$ with $|z| \geq 1$.

Now, we set

$$
b_{0}(z):=\chi(z) a(z)+(1-\chi(z)) a_{0}(z) .
$$

Since $\chi \in \mathrm{GS}_{\rho}^{0, \omega}$ and $a, a_{0} \in \mathrm{GS}_{\rho}^{m, \omega}$, we have $b_{0} \in \mathrm{GS}_{\rho}^{m, \omega}$. As $\chi(z)=0$ for all $z \notin \Gamma$, we obtain (since $a_{0}$ satisfies (4.29) for all $|z| \geq R$ ),

$$
\left|b_{0}(z)\right|=\left|a_{0}(z)\right| \geq C_{1} e^{m \omega(z)}, \quad z \notin \Gamma,|z| \geq R .
$$

On the other hand, since $a^{\prime}, a_{0}^{\prime} \geq 0$ and $0 \leq \chi \leq 1$, we have from (4.32) and (4.34), where $\bar{C}>0$ is as in (4.33) and $C^{\prime}>0$ is as in (4.35),

$$
\begin{aligned}
\left|b_{0}(z)\right| & =\left|\chi(z) a^{\prime}(z)+\chi(z) a^{\prime \prime}(z)+(1-\chi(z)) a_{0}^{\prime}(z)+(1-\chi(z)) a_{0}^{\prime \prime}(z)\right| \\
& \geq \chi(z) a^{\prime}(z)+(1-\chi(z)) a_{0}^{\prime}(z)-\chi(z)\left|a^{\prime \prime}(z)\right|-(1-\chi)(z)\left|a_{0}^{\prime \prime}(z)\right| \\
& \geq C_{1} e^{m \omega(z)}-\left(\bar{C}+C^{\prime}\right)\langle z\rangle^{-\rho} e^{m \omega(z)} \\
& \geq \frac{C_{1}}{2} e^{m \omega(z)}, \quad z \in \Gamma,\langle z\rangle \geq \max \left\{R,\left(2\left(\bar{C}+C^{\prime}\right) / C_{1}\right)^{1 / \rho}\right\} .
\end{aligned}
$$

Hence, we obtain

$$
\left|b_{0}(z)\right| \geq \frac{C_{1}}{2} e^{m \omega(z)} \geq \frac{C_{1}}{2} e^{-|m| \omega(z)}, \quad\langle z\rangle \geq \max \left\{R,\left(2\left(\bar{C}+C^{\prime}\right) / C_{1}\right)^{1 / \rho}\right\},
$$


and we have condition $(i)$ of Theorem 3.3 for $b_{0}$.

Since $\chi$ is as in Lemma 4.16, there exists $C>0$ such that, for the previous $n \in \mathbb{N}$,

$$
\left|D^{\alpha} \chi(z)\right| \leq C\langle z\rangle^{-|\alpha|} e^{\varphi_{\sigma}^{*}(|\alpha|)} \leq C\langle z\rangle^{-\rho|\alpha|} e^{\frac{1}{n} \varphi_{\sigma}^{*}(n|\alpha|)},
$$

for all $\alpha \in \mathbb{N}_{0}^{2 d}, z \in \mathbb{R}^{2 d}$. The same estimate is also valid for $1-\chi$ (probably with a change of the constant $C>0$ ) for all $\alpha \in \mathbb{N}_{0}^{2 d}$ and $z \in \mathbb{R}^{2 d}$. Therefore, since $a, a_{0}$ satisfy (4.30) for the same $C_{2} \geq 1, n \in \mathbb{N}$ and in $\Gamma \backslash B(0, R)$, by Leibniz rule we have for all $\alpha \in \mathbb{N}_{0}^{2 d}$ and $z \in \Gamma,|z| \geq R$,

$$
\begin{aligned}
\left|D^{\alpha} b_{0}(z)\right| \leq & \sum_{\beta \leq \alpha}\left(\begin{array}{c}
\alpha \\
\beta
\end{array}\right)\left(\left|D^{\beta} \chi(z)\right|\left|D^{\alpha-\beta} a(z)\right|+\left|D^{\beta}(1-\chi)(z)\right|\left|D^{\alpha-\beta} a_{0}(z)\right|\right) \\
\leq & \sum_{\beta \leq \alpha}\left(\begin{array}{c}
\alpha \\
\beta
\end{array}\right) C\langle z\rangle^{-\rho|\beta|} e^{\frac{1}{n} \varphi_{\sigma}^{*}(n|\beta|)} \times \\
& \times C_{2}^{|\alpha-\beta|}\langle z\rangle^{-\rho|\alpha-\beta|} e^{\frac{1}{n} \varphi_{\sigma}^{*}(n|\alpha-\beta|)}\left(|a(z)|+\left|a_{0}(z)\right|\right) .
\end{aligned}
$$

Since $a, a_{0} \in \mathrm{GS}_{\rho}^{m, \omega}$, there exists $C^{\prime}>0$ such that, using (0.11), (since $\left.\sum_{\beta \leq \alpha}\left(\begin{array}{l}\alpha \\ \beta\end{array}\right)=2^{|\alpha|}\right)$,

$$
\left|D^{\alpha} b_{0}(z)\right| \leq C\left(2 C_{2}\right)^{|\alpha|}\langle z\rangle^{-\rho|\alpha|} e^{\frac{1}{n} \varphi_{\sigma}^{*}(n|\alpha|)} 2 C^{\prime} e^{m \omega(z)} .
$$

Take $D=2 C_{2} \max \left\{1,4 C C^{\prime} / C_{1}\right\}>0$. Then, from (4.36) we obtain

$$
\begin{aligned}
\left|D^{\alpha} b_{0}(z)\right| & \leq D^{|\alpha|}\langle z\rangle^{-\rho|\alpha|} e^{\frac{1}{n} \varphi_{\sigma}^{*}(n|\alpha|)} \frac{C_{1}}{2} e^{m \omega(z)} \\
& \leq D^{|\alpha|}\langle z\rangle^{-\rho|\alpha|} e^{\frac{1}{n} \varphi_{\sigma}^{*}(n|\alpha|)}\left|b_{0}(z)\right|
\end{aligned}
$$

for all $\alpha \in \mathbb{N}_{0}^{2 d}$ and $z \in \Gamma$ with $|z|$ large enough. On the other hand, if $z \notin \Gamma$, then by construction $b_{0}(z)=a_{0}(z)$, thus, since $a_{0}$ satisfies $(4.30)$ for $C_{2}>0$ and $n \in \mathbb{N}$,

$$
\begin{aligned}
\left|D^{\alpha} b_{0}(z)\right|=\left|D^{\alpha} a_{0}(z)\right| & \leq C_{2}^{|\alpha|}\langle z\rangle^{-\rho|\alpha|} e^{\frac{1}{n} \varphi_{\sigma}^{*}(n|\alpha|)}\left|a_{0}(z)\right| \\
& \leq D^{|\alpha|}\langle z\rangle^{-\rho|\alpha|} e^{\frac{1}{n} \varphi_{\sigma}^{*}(n|\alpha|)}\left|b_{0}(z)\right|,
\end{aligned}
$$

for all $\alpha \in \mathbb{N}_{0}^{2 d}$ and $z \notin \Gamma,|z| \geq R$. Hence, $b_{0}$ satisfies condition $(i i)$ of Theorem 3.3 for all $z \in \mathbb{R}^{2 d}$ with $|z|$ large enough.

We deduce from Corollary 3.5 (see also Theorem 3.3) that there exists $c \in$ $\mathrm{GS}_{\rho}^{|m|, \omega}$ such that

$$
c \# b_{0}=1+s
$$


for some $s \in \mathcal{S}_{\omega}\left(\mathbb{R}^{2 d}\right)$. Therefore,

$$
\begin{aligned}
b & =b \# c \# b_{0}-b \# s \\
& =b \# c \#\left(b_{0}-a\right)+b \# c \# a-b \# s .
\end{aligned}
$$

So, we obtain

$$
\begin{aligned}
b^{w}(x, D) u= & b^{w}(x, D) c^{w}(x, D)\left(b_{0}-a\right)^{w}(x, D) u+ \\
& +b^{w}(x, D) c^{w}(x, D) a^{w}(x, D) u-b^{w}(x, D) s^{w}(x, D) u .
\end{aligned}
$$

We claim that $b^{w}(x, D) u \in \mathcal{S}_{\omega}\left(\mathbb{R}^{d}\right)$. Since $b(z)=0$ for all $z \notin \Gamma^{\prime}$ and

$$
b_{0}-a=\chi a+(1-\chi) a_{0}-a=(1-\chi)\left(a_{0}-a\right)
$$

vanishes for $z \in \Gamma^{\prime},|z| \geq 1$ (because $\chi(z)=1$ ) we deduce that

$$
E:=\operatorname{supp}(b) \cap \operatorname{supp}\left(b_{0}-a\right)
$$

is a compact set. This implies

$$
b \# c \#\left(b_{0}-a\right) \in \mathcal{S}_{\omega}\left(\mathbb{R}^{2 d}\right) .
$$

Indeed, let $\tilde{\chi} \in \mathcal{D}_{(\omega)}\left(\mathbb{R}^{2 d}\right)$, with $\tilde{\chi}=1$ on $E$. Then, $b \# c \#\left(b_{0}-a\right)$ has the same asymptotic expansion of $b \# c \#\left(\widetilde{\chi}\left(b_{0}-a\right)\right)$. Then, by Proposition 2.3,

$$
b^{w}(x, D) c^{w}(x, D)\left(b_{0}-a\right)^{w}(x, D)=b^{w}(x, D) c^{w}(x, D)\left(\widetilde{\chi}\left(b_{0}-a\right)\right)^{w}(x, D)+R,
$$

for an $\omega$-regularizing operator $R$. Since $b_{0}-a \in \mathrm{GS}_{\rho}^{m, \omega}$, we use Lemma 2.8, and then, as $\tilde{\chi} \in \mathcal{D}_{(\omega)}\left(\mathbb{R}^{2 d}\right)$, we can reproduce Lemma $1.11(a)$. Therefore by Proposition 1.19,

$$
\left(\widetilde{\chi}\left(b_{0}-a\right)\right)^{w}(x, D) u \in \mathcal{S}_{\omega}\left(\mathbb{R}^{d}\right), \quad u \in \mathcal{S}_{\omega}^{\prime}\left(\mathbb{R}^{d}\right) .
$$

The continuity of the Weyl operator yields

$$
c^{w}(x, D)\left(\widetilde{\chi}\left(b_{0}-a\right)\right)^{w}(x, D) u \in \mathcal{S}_{\omega}\left(\mathbb{R}^{d}\right), \quad u \in \mathcal{S}_{\omega}^{\prime}\left(\mathbb{R}^{d}\right),
$$

and

$$
b^{w}(x, D) c^{w}(x, D)\left(\widetilde{\chi}\left(b_{0}-a\right)\right)^{w}(x, D) u \in \mathcal{S}_{\omega}\left(\mathbb{R}^{d}\right), \quad u \in \mathcal{S}_{\omega}^{\prime}\left(\mathbb{R}^{d}\right) .
$$

Hence, by (4.38)

$$
b^{w}(x, D) c^{w}(x, D)\left(b_{0}-a\right)^{w}(x, D) u \in \mathcal{S}_{\omega}\left(\mathbb{R}^{d}\right), \quad u \in \mathcal{S}_{\omega}^{\prime}\left(\mathbb{R}^{d}\right) .
$$


By assumption, $a^{w}(x, D) u \in \mathcal{S}_{\omega}\left(\mathbb{R}^{d}\right)$, so as before we deduce

$$
b^{w}(x, D) c^{w}(x, D) a^{w}(x, D) u \in \mathcal{S}_{\omega}\left(\mathbb{R}^{d}\right), \quad u \in \mathcal{S}_{\omega}^{\prime}\left(\mathbb{R}^{d}\right) .
$$

Furthermore, since $s \in \mathcal{S}_{\omega}\left(\mathbb{R}^{2 d}\right)$, we have

$$
b^{w}(x, D) s^{w}(x, D) u \in \mathcal{S}_{\omega}\left(\mathbb{R}^{d}\right), \quad u \in \mathcal{S}_{\omega}^{\prime}\left(\mathbb{R}^{d}\right) .
$$

Hence, by (4.37) we obtain that $b^{w}(x, D) u \in \mathcal{S}_{\omega}\left(\mathbb{R}^{d}\right)$ for all $u \in \mathcal{S}_{\omega}^{\prime}\left(\mathbb{R}^{d}\right)$ and the proof is complete.

Now, for certain weight functions $\omega$, we want to compare the global wave front sets appearing in Definitions 4.1 and 4.12 for every ultradistribution $u$ in $\mathcal{S}_{\omega}^{\prime}\left(\mathbb{R}^{d}\right)$. In fact, for weight functions as in Definition 4.13 we have

Theorem 4.18. Let $\omega$ be a $\rho$-regular weight function, for some $0<\rho \leq 1$. Then,

$$
\mathrm{WF}_{\omega}^{\prime}(u) \subset \mathrm{WF}_{\rho}^{\omega}(u), \quad u \in \mathcal{S}_{\omega}^{\prime}\left(\mathbb{R}^{d}\right) .
$$

Proof. Let $0 \neq z_{0} \notin \mathrm{WF}_{\rho}^{\omega}(u)$. By Proposition 4.17, there exist $b \in \mathrm{GS}_{\rho}^{0, \omega}$ and an open conic set $\Gamma \subset \mathbb{R}^{2 d} \backslash\{0\}$ such that $z_{0} \in \Gamma, 0 \leq b \leq 1, b(z)=1$ for $z \in \Gamma$ with $|z| \geq 1$ and $b^{w}(x, D) u \in \mathcal{S}_{\omega}\left(\mathbb{R}^{d}\right)$. Put $\widetilde{b}:=1-b$. We have $\widetilde{b} \in \operatorname{GS}_{\rho}^{0, \omega}$, $\widetilde{b}(z)=0$ for $z \in \Gamma$ with $|z| \geq 1$, so in particular $z_{0} \notin \operatorname{conesupp}(\widetilde{b})$. Since $b^{w}(x, D) u \in \mathcal{S}_{\omega}\left(\mathbb{R}^{d}\right)$ we obtain, by Lemma 4.3 and Proposition 4.9,

$$
\mathrm{WF}_{\omega}^{\prime}(u)=\mathrm{WF}_{\omega}^{\prime}\left(b^{w}(x, D) u+\widetilde{b}^{w}(x, D) u\right)=\mathrm{WF}_{\omega}^{\prime}\left(\widetilde{b}^{w}(x, D) u\right) \subset \operatorname{conesupp}(\widetilde{b}) .
$$

Hence $z_{0} \notin \mathrm{WF}_{\omega}^{\prime}(u)$.

Theorem 4.19. Let $\omega$ be a weight function. If for some $0<\rho \leq 1$,

$$
\omega\left(t^{1 / \rho}\right)=o(\sigma(t)) \text { and } \sigma\left(t^{1+\rho / 2}\right)=O(\gamma(t)) \text { as } t \rightarrow \infty,
$$

for some Gevrey weight function $\sigma$ and some weight function $\gamma$, then

$$
\mathrm{WF}_{\rho}^{\omega}(u) \subset \mathrm{WF}_{\omega}^{\prime}(u), \quad u \in \mathcal{S}_{\omega}^{\prime}\left(\mathbb{R}^{d}\right) .
$$

Remark 4.20. The assumption (4.39) in Theorem 4.19 implies

$$
\omega\left(t^{(2+\rho) /(2 \rho)}\right)=o(\gamma(t)), \quad t \rightarrow \infty,
$$

for some weight function $\gamma$. For $\omega(t)=t^{a}, a=1-\rho$, this condition implies (as $\gamma(t)=o(t)$ as $t \rightarrow \infty)$

$$
a \frac{2+\rho}{2 \rho}=a \frac{3-a}{2(1-a)}<1,
$$


or, equivalently,

$$
0<a<\frac{5-\sqrt{17}}{2}, \quad \frac{-3+\sqrt{17}}{2}<\rho<1 .
$$

Proof of Theorem 4.19. Take

$$
\psi(z)=e^{-|z|^{2} / 2}, \quad z \in \mathbb{R}^{2 d},
$$

which belongs to $\mathcal{S}_{\omega}\left(\mathbb{R}^{2 d}\right)$, for all weight function $\omega$ (by the estimates in Definition 0.13). As in [61, Theorem 4.2], the Wigner transform of $\psi$ (Definition 0.34) is

$$
\operatorname{Wig}(\psi)(z)=(4 \pi)^{d / 2} e^{-|z|^{2}}, \quad z \in \mathbb{R}^{2 d} .
$$

Let $0 \neq z_{0} \notin \mathrm{WF}_{\omega}^{\prime}(u)$. Then, there exists an open conic set $\Gamma \subseteq \mathbb{R}^{2 d} \backslash\{0\}$ such that $z_{0} \in \Gamma$ and

$$
\sup _{z \in \Gamma} e^{\lambda \omega(z)}\left|V_{\psi} u(z)\right|<\infty, \quad \lambda>0 .
$$

Take $\Gamma^{\prime} \subseteq \mathbb{R}^{2 d} \backslash\{0\}$ an open conic set such that $z_{0} \in \Gamma^{\prime}$ and $\overline{\Gamma^{\prime} \cap S_{2 d-1}} \subseteq \Gamma$. For the weight function $\gamma$, by Lemma 4.16 there exists $b \in \mathrm{GS}_{1}^{0, \gamma}$ such that $0 \leq b \leq 1, \operatorname{supp}(b) \subseteq \Gamma$, and $b(z)=1$ for $z \in \Gamma^{\prime},|z| \geq 1$.

We define

$$
a:=b * \operatorname{Wig}(\psi) .
$$

To estimate the derivatives of $a$, we use the fact that $b \in \mathrm{GS}_{1}^{0, \gamma}$, Lemma 0.2 and (0.10) to obtain that for all $\lambda>0$ there exists $C_{\lambda}>0$ such that

$$
\begin{aligned}
\left|D^{\alpha} a(z)\right| & \leq \int_{\mathbb{R}^{2 d}}\left|D_{z}^{\alpha} b(z-w)\right| \operatorname{Wig}(\psi)(w) d w \\
& \leq \int_{\mathbb{R}^{2 d}} C_{\lambda}\langle z-w\rangle^{-|\alpha|} e^{\lambda L \varphi_{\gamma}^{*}\left(\frac{|\alpha|}{\lambda L}\right)}(4 \pi)^{d / 2} e^{-|w|^{2}} d w \\
& \leq \int_{\mathbb{R}^{2 d}} C_{\lambda}\langle z-w\rangle^{-\rho|\alpha|} e^{\lambda L \varphi_{\gamma}^{*}\left(\frac{|\alpha|}{\lambda L}\right)}(4 \pi)^{d / 2} e^{-|w|^{2}} d w \\
& \leq C_{\lambda}(4 \pi)^{d / 2}\langle z\rangle^{-\rho|\alpha|}\left(\sqrt{2}^{\rho|\alpha|} e^{\lambda L \varphi_{\gamma}^{*}\left(\frac{|\alpha|}{\lambda L}\right)}\right) \int_{\mathbb{R}^{2 d}}\langle w\rangle^{\rho|\alpha|} e^{-|w|^{2}} d w \\
& \leq C_{\lambda}(4 \pi)^{d / 2}\langle z\rangle^{-\rho|\alpha|} e^{\lambda L} e^{\lambda \varphi_{\gamma}^{*}\left(\frac{|\alpha|}{\lambda}\right)} \int_{\mathbb{R}^{2 d}}\left(\langle w\rangle^{2}\right)^{|\alpha|(\rho / 2)} e^{-|w|^{2}} d w
\end{aligned}
$$

for all $\alpha \in \mathbb{N}_{0}^{2 d}$ and $z \in \mathbb{R}^{2 d}$. Then, by $(0.7)$,

$$
\int_{\mathbb{R}^{2 d}}\left(\langle w\rangle^{2}\right)^{|\alpha|(\rho / 2)} e^{-|w|^{2}} d w \leq e^{\lambda(\rho / 2) \varphi_{\gamma}^{*}\left(\frac{|\alpha|}{\lambda}\right)} \int_{\mathbb{R}^{2 d}} e^{\lambda(\rho / 2) \gamma\left(\langle w\rangle^{2}\right)} e^{-|w|^{2}} d w .
$$


In particular, as $\gamma(t)=o(t), t \rightarrow \infty$, there exists $D_{\lambda}>0$ such that

$$
\lambda(\rho / 2) \gamma\left(\langle w\rangle^{2}\right) \leq \frac{1}{2}|w|^{2}+D_{\lambda}, \quad w \in \mathbb{R}^{2 d},
$$

and then the integral is convergent. So, from (4.41), using Lemma 0.10(1) (see Remark 4.20) we obtain that for all $\lambda>0$ there exists $C_{\lambda}^{\prime}>0$ such that

$$
\begin{aligned}
\left|D^{\alpha} a(z)\right| & \leq C_{\lambda}^{\prime}\langle z\rangle^{-\rho|\alpha|} e^{\lambda(1+\rho / 2) \varphi_{\gamma}^{*}\left(\frac{|\alpha|}{\lambda}\right)} \\
& \leq C_{\lambda}^{\prime \prime}\langle z\rangle^{-\rho|\alpha|} e^{\lambda \rho \varphi_{\omega}^{*}\left(\frac{|\alpha|}{\lambda}\right)},
\end{aligned}
$$

for some constant $C_{\lambda}^{\prime \prime}>0$ depending on $\lambda>0$. This shows $a \in \operatorname{GS}_{\rho}^{0, \omega}$.

Let $\Gamma^{\prime \prime} \subseteq \Gamma^{\prime}$ be another open conic set such that $z_{0} \in \Gamma^{\prime \prime}$ and $\overline{\Gamma^{\prime \prime} \cap S_{2 d-1}} \subseteq \Gamma^{\prime}$. Then, there exists $\delta>0$ (see $[14,(3.25)]$ ) such that $z-w / t \in \Gamma^{\prime}$ for $z \in \Gamma^{\prime \prime}$ with $|z|=1,|w| \leq \delta$, and $t \geq 1$. Since $|z-w| \geq|z|-\delta \geq 1$ holds if $|w| \leq \delta$ and $|z| \geq 1+\delta$, we have for $z \in \Gamma^{\prime \prime},|z| \geq 1+\delta,\left(\right.$ as $b(z)=1$ for $\left.z \in \Gamma^{\prime},|z| \geq 1\right)$

$$
\begin{aligned}
|a(z)| & =\int_{\mathbb{R}^{2 d}} b(z-w) \operatorname{Wig}(\psi)(w) d w \\
& \geq \int_{\{|w| \leq \delta\}} b\left(|z|\left(\frac{z}{|z|}-\frac{w}{|z|}\right)\right) \operatorname{Wig}(\psi)(w) d w \\
& =\int_{\{|w| \leq \delta\}} \operatorname{Wig}(\psi)(w) d w=: C^{*}>0 .
\end{aligned}
$$

Hence (4.29) is satisfied for $m=0$. Moreover, as $\sigma\left(t^{1+\rho / 2}\right)=O(\gamma(t)), t \rightarrow \infty$, we use Lemma $0.10(2)$ to obtain, by (4.42), that there exist $C^{\prime}>0$ and $n \in \mathbb{N}$ such that for $\alpha \in \mathbb{N}_{0}^{2 d}$ and $z \in \Gamma^{\prime \prime},|z| \geq 1+\delta$,

$$
\left|D^{\alpha} a(z)\right| \leq C^{\prime}\langle z\rangle^{-\rho|\alpha|} e^{\frac{1}{n} \varphi_{\sigma}^{*}(n|\alpha|)} \leq \frac{C^{\prime}}{C^{*}}\langle z\rangle^{-\rho|\alpha|} e^{\frac{1}{n} \varphi_{\sigma}^{*}(n|\alpha|)}|a(z)|,
$$

so (4.30) is satisfied, too. Thus $z_{0}$ is non-characteristic for $a$.

It only remains to show that $a^{w}(x, D) u \in \mathcal{S}_{\omega}\left(\mathbb{R}^{d}\right)$. We recall that the Weyl operator $a^{w}(x, D)$ can be written as (see for instance $[29,(6),(3)]$ )

$$
a^{w}(x, D) u(x)=\int_{\mathbb{R}^{2 d}} b(z) V_{\psi} u(z) \Pi(z) \psi(x) d z, \quad x \in \mathbb{R}^{d} .
$$


Since $\operatorname{supp}(b) \subseteq \Gamma$ and $0 \leq b \leq 1$, given $\alpha \in \mathbb{N}_{0}^{d}$ we have by (4.43), for $z=(t, \xi) \in \mathbb{R}^{2 d}$,

$$
\begin{aligned}
\left|D^{\alpha} a^{w}(x, D) u(x)\right| & \leq \int_{\Gamma}\left|V_{\psi} u(t, \xi)\right|\left|D_{x}^{\alpha}\left(e^{i x \cdot \xi} \psi(x-t)\right)\right| d t d \xi \\
& \leq \sum_{\beta \leq \alpha}\left(\begin{array}{c}
\alpha \\
\beta
\end{array}\right) \int_{\Gamma}\left|V_{\psi} u(t, \xi)\right||\xi|^{|\beta|}\left|D_{x}^{\alpha-\beta} \psi(x-t)\right| d t d \xi
\end{aligned}
$$

From (4.40), (0.7) and since $\psi \in \mathcal{S}_{\omega}\left(\mathbb{R}^{d}\right)$, for all $\lambda>0$ there exist $C_{\lambda}, C_{\lambda}^{\prime}>0$ (different from the previous ones) such that (assuming $|\xi| \geq 1$ )

$$
\begin{aligned}
\left|D^{\alpha} a^{w}(x, D) u(x)\right| \leq & \sum_{\beta \leq \alpha}\left(\begin{array}{c}
\alpha \\
\beta
\end{array}\right) \int_{\Gamma} C_{\lambda} e^{-2(\lambda L+1) \omega(t, \xi)} e^{\lambda L \varphi_{\omega}^{*}\left(\frac{|\beta|}{\lambda L}\right)} e^{\lambda L \omega(\xi)} \times \\
& \times C_{\lambda}^{\prime} e^{\lambda L \varphi_{\omega}^{*}\left(\frac{|\alpha-\beta|}{\lambda L}\right)} e^{-\lambda L \omega(x-t)} d t d \xi .
\end{aligned}
$$

By (0.1),

$$
\begin{aligned}
-2(\lambda L+1) \omega(t, \xi) & \leq-(\lambda L+1)(\omega(t)+\omega(\xi)) \\
& \leq-(\omega(t)+\omega(\xi))-\lambda L \omega(\xi)+\lambda L \omega(x-t)-\lambda \omega(x)+\lambda L .
\end{aligned}
$$

So, we have

$$
-2(\lambda L+1) \omega(t, \xi)+\lambda L \omega(\xi)-\lambda L \omega(x-t) \leq-(\omega(t)+\omega(\xi))-\lambda \omega(x)+\lambda L,
$$

and therefore

$$
\begin{aligned}
\left|D^{\alpha} a^{w}(x, D) u(x)\right| \leq & C_{\lambda} C_{\lambda}^{\prime} e^{\lambda L} e^{-\lambda \omega(x)} \sum_{\beta \leq \alpha}\left(\begin{array}{c}
\alpha \\
\beta
\end{array}\right) e^{\lambda L \varphi_{\omega}^{*}\left(\frac{|\beta|}{\lambda L}\right)} e^{\lambda L \varphi_{\omega}^{*}\left(\frac{|\alpha-\beta|}{\lambda L}\right)} \times \\
& \times \int_{\mathbb{R}^{2 d}} e^{-\omega(t)-\omega(\xi)} d t d \xi
\end{aligned}
$$

By Lemma 0.8, $\sum_{\beta \leq \alpha}\left(\begin{array}{l}\alpha \\ \beta\end{array}\right) e^{\lambda L \varphi_{\omega}^{*}\left(\frac{|\beta|}{\lambda L}\right)} e^{\lambda L \varphi_{\omega}^{*}\left(\frac{|\alpha-\beta|}{\lambda L}\right)} \leq e^{\lambda \varphi_{\omega}^{*}\left(\frac{|\alpha|}{\lambda}\right)} e^{\lambda L}$, and we get $a^{w}(x, D) u \in \mathcal{S}_{\omega}\left(\mathbb{R}^{d}\right)$. Then, $z_{0} \notin \mathrm{WF}_{\rho}^{\omega}(u)$.

Corollary 4.21. Let $\omega$ be a $\rho$-regular weight function for some $0<\rho \leq 1$ that satisfies (4.39) for two weight functions $\sigma$ and $\gamma$ as in Theorem 4.19. Then,

$$
\mathrm{WF}_{\rho}^{\omega}(u)=\mathrm{WF}_{\omega}^{\prime}(u), \quad u \in \mathcal{S}_{\omega}^{\prime}\left(\mathbb{R}^{d}\right) .
$$

Proposition 4.22. Let $w \in \mathbb{R}^{2 d}$. Under the hypotheses of Corollary 4.21, we have

$$
\mathrm{WF}_{\rho}^{\omega}(\Pi(w) u)=\mathrm{WF}_{\rho}^{\omega}(u), \quad u \in \mathcal{S}_{\omega}^{\prime}\left(\mathbb{R}^{d}\right) .
$$


Proof. It follows from [14, Proposition 3.19].

Example 4.23. Let $(-3+\sqrt{17}) / 2<\rho<1$ and let $a=1-\rho$. Then, for every $b, c>0$ such that

$$
\frac{1-\rho}{\rho}<b<\frac{2}{2+\rho} \text { and } b \frac{2+\rho}{2}<c<1
$$

the weight functions $\omega(t)=t^{a}, \sigma(t)=t^{b}$ and $\gamma(t)=t^{c}$ satisfy the hypotheses of Corollary 4.21 (see Remark 4.20).

\subsection{Regularity of Weyl quantizations}

In this section we study the regularity of Weyl quantizations with the Weyl wave front set with symbols in the class $\mathrm{GS}_{\rho}^{m, \omega}$.

Lemma 4.24. Let $\omega$ be a $\rho$-regular weight function for some $0<\rho \leq 1$ and $u \in \mathcal{S}_{\omega}^{\prime}\left(\mathbb{R}^{d}\right)$. Then $\mathrm{WF}_{\rho}^{\omega}(u)$ is empty if and only if $u \in \mathcal{S}_{\omega}\left(\mathbb{R}^{d}\right)$.

Proof. Let us first assume that $u \in \mathcal{S}_{\omega}\left(\mathbb{R}^{d}\right)$. Taking $a \equiv 1 \in \mathrm{GS}_{\rho}^{0, \omega}$ we have that $z$ is non-characteristic for $a$ for every $z \in \mathbb{R}^{2 d} \backslash\{0\}$, and $a^{w}(x, D) u=u \in$ $\mathcal{S}_{\omega}\left(\mathbb{R}^{d}\right)$, so $\mathrm{WF}_{\rho}^{\omega}(u)$ is empty.

On the other hand, if $\mathrm{WF}_{\rho}^{\omega}(u)$ is empty, then from Theorem 4.18 we have that $\mathrm{WF}_{\omega}^{\prime}(u)$ is empty, and then from [14, Proposition 3.18] we obtain $u \in$ $\mathcal{S}_{\omega}\left(\mathbb{R}^{d}\right)$.

Proposition 4.25. Let $\omega$ be a $\rho$-regular weight function for some $0<\rho \leq 1$ and let $m \in \mathbb{R}$. For a global symbol $a \in \mathrm{GS}_{\rho}^{m, \omega}$, we have

$$
\mathrm{WF}_{\rho}^{\omega}(u) \subset \mathrm{WF}_{\rho}^{\omega}\left(a^{w}(x, D) u\right) \cup \operatorname{char}(a), \quad u \in \mathcal{S}_{\omega}^{\prime}\left(\mathbb{R}^{d}\right) .
$$

Proof. Let $0 \neq z_{0} \notin \mathrm{WF}_{\rho}^{\omega}\left(a^{w}(x, D) u\right) \cup \operatorname{char}(a)$. By Proposition 4.17 we have that there exist $b \in \mathrm{GS}_{\rho}^{0, \omega}$ and an open conic set $\Gamma \subset \mathbb{R}^{2 d} \backslash\{0\}$ containing $z_{0}$ such that $0 \leq b \leq 1, b(z)=1$ for $z \in \Gamma,|z| \geq 1$, and

$$
b^{w}(x, D) a^{w}(x, D) u \in \mathcal{S}_{\omega}\left(\mathbb{R}^{d}\right), \quad u \in \mathcal{S}_{\omega}^{\prime}\left(\mathbb{R}^{d}\right) .
$$

The Weyl product $b \# a$ of the composition $b^{w}(x, D) a^{w}(x, D)$ has an asymptotic expansion $\sum c_{j}(x, \xi)$ as in formula (3.5). Then, by Theorem 2.6 there exists 
$c \in \mathrm{GS}_{\rho}^{m, \omega}$ such that $c \sim \sum c_{j}$ and, from (2.12),

$$
c(x, \xi)=b(x, \xi) a(x, \xi)+\sum_{n=1}^{\infty} \sum_{j=j_{n}}^{j_{n+1}-1} \Psi_{j, n}(x, \xi) c_{j}(x, \xi),
$$

where $\left(\Psi_{j, n}\right)$ is defined in $(2.4)$ (see the proof of Theorem 2.6 for the conditions on the sequence $\left.\left(j_{n}\right)\right)$. From the properties of $b$, we have

$$
c(z)=a(z) \quad z \in \Gamma,|z| \geq 1 .
$$

Now, since $z_{0} \notin \operatorname{char}(a)$ there exists an open conic set $\Gamma^{\prime} \subset \mathbb{R}^{2 d} \backslash\{0\}$ such that $a$ satisfies (4.29) and (4.30) for all $\alpha \in \mathbb{N}_{0}^{2 d}, z \in \Gamma^{\prime},|z| \geq 1$. Thus, from (4.46) we have that $z_{0}$ is non-characteristic for $c$ (in probably another open conic set $\Gamma^{\prime \prime}$ satisfying $\left.\overline{\Gamma^{\prime \prime} \cap S_{2 d-1}} \subseteq \Gamma \cap \Gamma^{\prime}\right)$. Finally, by construction we have

$$
b^{w}(x, D) a^{w}(x, D) u=c^{w}(x, D) u+R u, \quad u \in \mathcal{S}_{\omega}^{\prime}\left(\mathbb{R}^{d}\right),
$$

where $R$ is an $\omega$-regularizing operator. Then, using (4.44) we obtain that $c^{w}(x, D) u \in \mathcal{S}_{\omega}\left(\mathbb{R}^{d}\right)$ and therefore $z_{0} \notin \mathrm{WF}_{\rho}^{\omega}(u)$.

Lemma 4.26. Let $\omega$ be a $\rho$-regular weight function for some $0<\rho \leq 1$. We have

$$
\mathrm{WF}_{\rho}^{\omega}(u)=\mathrm{WF}_{\rho}^{\omega}(u+v), \quad u \in \mathcal{S}_{\omega}^{\prime}\left(\mathbb{R}^{d}\right), v \in \mathcal{S}_{\omega}\left(\mathbb{R}^{d}\right) .
$$

Proof. Let $0 \neq z_{0} \notin \mathrm{WF}_{\rho}^{\omega}(u)$. Then there exists a symbol $a \in \mathrm{GS}_{\rho}^{m, \omega}$ for some $m \in \mathbb{R}$ such that $z_{0}$ is non-characteristic for $a$ and $a^{w}(x, D) u \in \mathcal{S}_{\omega}\left(\mathbb{R}^{d}\right)$. Since $v \in \mathcal{S}_{\omega}\left(\mathbb{R}^{d}\right)$ we have by Lemma 2.8 and Theorem 1.15 that $a^{w}(x, D)(u+v) \in$ $\mathcal{S}_{\omega}\left(\mathbb{R}^{d}\right)$, and therefore $z_{0} \notin \mathrm{WF}_{\rho}^{\omega}(u+v)$. We then obtain

$$
\mathrm{WF}_{\rho}^{\omega}(u+v) \subset \mathrm{WF}_{\rho}^{\omega}(u) .
$$

Proceeding in the same way, we get

$$
\mathrm{WF}_{\rho}^{\omega}(u)=\mathrm{WF}_{\rho}^{\omega}(u+v-v) \subset \mathrm{WF}_{\rho}^{\omega}(u+v),
$$

and the proof is complete.

Proposition 4.27. Let $\omega$ be a $\rho$-regular weight function for some $0<\rho \leq 1$. Let $m \in \mathbb{R}$ and $a \in \mathrm{GS}_{\rho}^{m, \omega}$. Then

$$
\mathrm{WF}_{\rho}^{\omega}\left(a^{w}(x, D) u\right) \subset \operatorname{conesupp}(a), \quad u \in \mathcal{S}_{\omega}^{\prime}\left(\mathbb{R}^{d}\right) .
$$


Proof. Let $0 \neq z_{0} \notin \operatorname{conesupp}(a)$. Then, there exists an open conic set $\Gamma \subset$ $\mathbb{R}^{2 d} \backslash\{0\}$ containing $z_{0}$ such that $a(z)=0$ for every $z \in \Gamma$ with $|z| \geq R$, for some $R>0$. We take an open conic set $\Gamma^{\prime} \subset \mathbb{R}^{2 d} \backslash\{0\}$ such that $\overline{\Gamma^{\prime} \cap S_{2 d-1}} \subseteq \Gamma$ and $z_{0} \in \Gamma^{\prime}$, and then consider $b \in \mathrm{GS}_{\rho}^{0, \omega}$ the function in Lemma 4.16. Since $b(z)=1$ for $z \in \Gamma^{\prime},|z| \geq 1$, it is clear that $b(z)$ satisfies (4.29) and (4.30), so $z_{0}$ is non-characteristic for $b$. As in the proof of Proposition 4.25, the Weyl product of the composition $b^{w}(x, D) a^{w}(x, D)$ has an asymptotic expansion $\sum c_{j}$ as in (3.5). By Theorem 2.6 there exists $c \in \mathrm{GS}_{\rho}^{m, \omega}$ such that $c \sim \sum c_{j}$ satisfying (4.47) for some $\omega$-regularizing operator $R$. Since supp $(b) \subseteq \Gamma$ and $a(z)=0$ for all $z \in \Gamma$ with $|z| \geq R$, we have that $\operatorname{supp}(a) \cap \operatorname{supp}(b)$ is compact. Therefore $\operatorname{supp}(c)$ is also compact and by Corollary $4.10, c^{w}(x, D)$ is $\omega$-regularizing. Then, for every $u \in \mathcal{S}_{\omega}^{\prime}\left(\mathbb{R}^{d}\right)$ we have

$$
b^{w}(x, D) a^{w}(x, D) u \in \mathcal{S}_{\omega}\left(\mathbb{R}^{d}\right),
$$

and hence $z_{0} \notin \mathrm{WF}_{\rho}^{\omega}\left(a^{w}(x, D) u\right)$.

Remark 4.28. We observe that, under the hypotheses in Corollary 4.21, we obtain Lemmas 4.24, 4.26 and Proposition 4.27 as an immediate application of [14, Proposition 3.18], Lemma 4.3, and Proposition 4.9.

However, in the proofs above the hypotheses in Corollary 4.21 were not necessary.

Proposition 4.29. Let $\omega$ be a $\rho$-regular weight function for some $0<\rho \leq 1$. Let $m \in \mathbb{R}$ and $a \in \mathrm{GS}_{\rho}^{m, \omega}$. Then,

$$
\mathrm{WF}_{\rho}^{\omega}\left(a^{w}(x, D) u\right) \subset \mathrm{WF}_{\rho}^{\omega}(u), \quad u \in \mathcal{S}_{\omega}^{\prime}\left(\mathbb{R}^{d}\right) .
$$

Proof. Take $0 \neq z_{0} \notin \mathrm{WF}_{\rho}^{\omega}(u)$. By Proposition 4.17, there exist $b \in \mathrm{GS}_{\rho}^{0, \omega}$ and an open conic set $\Gamma$ containing $z_{0}$ such that $b(z)=1$ for $z \in \Gamma,|z| \geq 1$ and $b^{w}(x, D) u \in \mathcal{S}_{\omega}\left(\mathbb{R}^{d}\right)$. Set $\tilde{b}=1-b \in \mathrm{GS}_{\rho}^{0, \omega}$. We have

$$
a^{w}(x, D) u=a^{w}(x, D) \tilde{b}^{w}(x, D) u+a^{w}(x, D) b^{w}(x, D) u, \quad u \in \mathcal{S}_{\omega}^{\prime}\left(\mathbb{R}^{d}\right) .
$$

By the continuity of the Weyl operator, $a^{w}(x, D) b^{w}(x, D) u \in \mathcal{S}_{\omega}\left(\mathbb{R}^{d}\right)$. On the other hand, proceeding as in the proof of Proposition 4.25 for the operator $a^{w}(x, D) \tilde{b}^{w}(x, D)$, there exists $c \in \operatorname{GS}_{\rho}^{m, \omega}$ satisfying (4.45) (replacing $b$ by $\tilde{b}$ ), where $\sum c_{j}$ is as in (3.5) (replacing $b$ by $\tilde{b}$ ). Since $\tilde{b}(z)=0$ for every $z \in \Gamma$, $|z| \geq 1$, we have that $c(z)$ vanishes for all $z \in \Gamma,|z| \geq 1$, and the Weyl symbol of $a^{w}(x, D) \tilde{b}^{w}(x, D)$ satisfies, similarly as (4.47),

$$
a^{w}(x, D) \tilde{b}^{w}(x, D) u=c^{w}(x, D) u+R u,
$$


for some $\omega$-regularizing operator $R$. Therefore, from (4.48), we obtain by Lemma 4.26 and Proposition 4.27,

$$
\mathrm{WF}_{\rho}^{\omega}\left(a^{w}(x, D) u\right)=\mathrm{WF}_{\rho}^{\omega}\left(c^{w}(x, D) u\right) \subset \operatorname{conesupp}(c) .
$$

Since $z_{0} \in \Gamma$ we have that $z_{0} \notin \operatorname{conesupp}(c)$ and then $z_{0} \notin \mathrm{WF}_{\rho}^{\omega}\left(a^{w}(x, D) u\right)$.

We have the following result as in [61, Proposition 2.11].

Corollary 4.30. Let $\omega$ be a $\rho$-regular weight function for some $0<\rho \leq 1$. Let $m \in \mathbb{R}$ and $a \in \mathrm{GS}_{\rho}^{m, \omega}$. If

$$
\text { conesupp }(a) \cap \mathrm{WF}_{\rho}^{\omega}(u)=\emptyset, \quad u \in \mathcal{S}_{\omega}^{\prime}\left(\mathbb{R}^{d}\right),
$$

then $a^{w}(x, D) u \in \mathcal{S}_{\omega}\left(\mathbb{R}^{d}\right)$.

Proof. By Propositions 4.27 and 4.29 we obtain $\mathrm{WF}_{\rho}^{\omega}\left(a^{w}(x, D) u\right)=\emptyset$. The result then follows by Lemma 4.24 .

By Propositions 4.25, 4.27, and 4.29 we have

Theorem 4.31. Let $\omega$ be a $\rho$-regular weight function for some $0<\rho \leq 1$. Let $m \in \mathbb{R}$ and $a \in \mathrm{GS}_{\rho}^{m, \omega}$. Then

$$
\begin{aligned}
\mathrm{WF}_{\rho}^{\omega}\left(a^{w}(x, D) u\right) & \subset \mathrm{WF}_{\rho}^{\omega}(u) \cap \operatorname{conesupp}(a) \\
& \subset \mathrm{WF}_{\rho}^{\omega}(u) \subset \mathrm{WF}_{\rho}^{\omega}\left(a^{w}(x, D) u\right) \cup \operatorname{char}(a)
\end{aligned}
$$

for all $u \in \mathcal{S}_{\omega}^{\prime}\left(\mathbb{R}^{d}\right)$.

Furthermore, by Corollary 4.21,

Corollary 4.32. Let $\omega$ be a $\rho$-regular weight function for some $0<\rho \leq 1$ which satisfies (4.39) for two weight functions $\sigma$ and $\gamma$ as in Theorem 4.19. Let $m \in \mathbb{R}$ and $a \in \mathrm{GS}_{\rho}^{m, \omega}$. Then

$$
\begin{aligned}
\mathrm{WF}_{\omega}^{\prime}\left(a^{w}(x, D) u\right) & \subset \mathrm{WF}_{\omega}^{\prime}(u) \cap \operatorname{conesupp}(a) \\
& \subset \mathrm{WF}_{\omega}^{\prime}(u) \subset \mathrm{WF}_{\omega}^{\prime}\left(a^{w}(x, D) u\right) \cup \operatorname{char}(a)
\end{aligned}
$$

for all $u \in \mathcal{S}_{\omega}^{\prime}\left(\mathbb{R}^{d}\right)$. 
Remark 4.33. Let $\omega$ be a $\rho$-regular weight function for some $0<\rho \leq 1$. Then, for all $m \in \mathbb{R}$ there exists $p \in \mathrm{GS}_{\rho}^{m, \omega}$ such that $z$ is non-characteristic for $p$ for all $z \in \mathbb{R}^{2 d} \backslash\{0\}$. Hence char $(p)=\emptyset$ and by Theorem 4.31,

$$
\mathrm{WF}_{\rho}^{\omega}\left(p^{w}(x, D) u\right)=\mathrm{WF}_{\rho}^{\omega}(u), \quad u \in \mathcal{S}_{\omega}^{\prime}\left(\mathbb{R}^{d}\right) .
$$

Example 4.34. Let $\omega(t)=t^{a}$ be a Gevrey weight function, $0<a<1 / 2$. From Example 4.14, $\omega$ is $(1-a)$-regular. Take $p$ as in (3.11). From (3.15) we deduce that every $z \in \mathbb{R}^{2 d} \backslash\{0\}$ is non-characteristic for $p$, and therefore the associated Weyl operator satisfies (4.49). Furthermore, if $0<a<(5-\sqrt{17}) / 2$, then by Corollary 4.21 ,

$$
\mathrm{WF}_{\omega}^{\prime}\left(p^{w}(x, D) u\right)=\mathrm{WF}_{\omega}^{\prime}(u), \quad u \in \mathcal{S}_{\omega}^{\prime}\left(\mathbb{R}^{d}\right) .
$$





\section{Index}

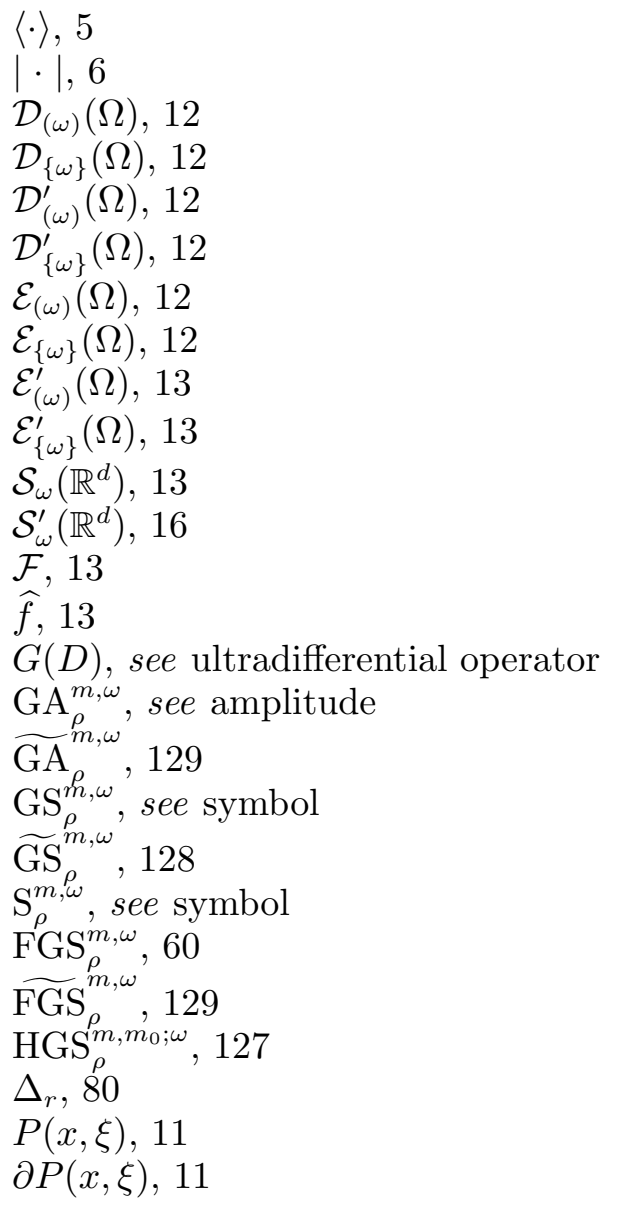

$V_{\psi}, 24$

$V_{\psi}^{*}, 25$

$T_{x}, 24$

$M_{\xi}, 24$

$\Pi(z), 24$

$T_{G}, 16$

$S_{2 d-1}, 139$

$\omega$, see weight function

$b^{w}(x, D), 106$

Wig, 27

$\varphi_{\omega}^{*}$, see Young conjugate

$\langle\cdot, \cdot\rangle, 24$

char $(a), 159$

conesupp $(u), 137$

$P_{\tau}, 106,130$

\#, 122

$|\cdot|_{\lambda}, 15$

| $\left.\cdot\right|_{K, \lambda}, 12$

$\|\cdot\|_{\omega, \lambda}, 26$

$\operatorname{supp}(T), 13$

$p_{\tau}, 105,130$

$p_{w}, 105$

$\mathrm{WF}_{\omega}^{\prime}(u), 137$

$\mathrm{WF}_{\rho}^{\omega}(u), 160$

amplitude global, 31

mixed, 129 
formal sum, 60

composition, 79

equivalent, 60

mixed, 129

equivalent, 130

transpose, 78

function

global ultradifferentiable, 13

test

of Beurling type, 12

of Roumieu type, 12

ultradifferentiable

of Beurling type, 12

of Roumieu type, 12

weight, 7

$\rho$-regular, 160

operator

global pseudodifferential, 35 $\omega$-regular, 116

kernel of, 43

modulation, 24

phase-shift, 24

regularizing, 52

translation, 24

ultradifferential, 16

Weyl, 106

parametrix, 116

point

characteristic, 159

non-characteristic, 159

quantization

$\tau, 106,130$

composition, 108

transpose, 108

support

conic, 137

distributions, 13

symbol $\omega$-hypoelliptic, 127

$\tau, 105,130$

global, 31

mixed, 128

of finite order, 31

Weyl, 105

transform

Fourier, 13

short-time Fourier, 24

Wigner, 27

ultradistribution

$\omega$-temperate, 16

of Beurling type, 12

of Roumieu type, 12

with compact support

of Beurling type, 13

of Roumieu type, 13

wave front set

$\omega, 137$

Weyl, 160

Young conjugate, 9 


\section{Bibliography}

[1] A. A. Albanese, D. Jornet, and A. Oliaro, Quasianalytic wave front sets for solutions of linear partial differential operators, Integral Equations Operator Theory 66 (2010), no. 2, 153-181.

[2] _ Wave front sets for ultradistribution solutions of linear partial differential operators with coefficients in non-quasianalytic classes, Math. Nachr. 285 (2012), no. 4, 411-425.

[3] E. Annovazzi, Esempi notevoli di funzioni gevrey ed ultradistribuzioni, Master's thesis, University of Torino, 1999/2000, Italian.

[4] V. Asensio, Quantizations and global hypoellipticity for pseudodifferential operators of infinite order in classes of ultradifferentiable functions, Preprint 2021, arXiv:2104.09198v1. Submitted for publication.

[5] V. Asensio, C. Boiti, D. Jornet, and A. Oliaro, Global wave front sets in ultradifferentiable classes, Preprint 2021. Submitted for publication.

[6] V. Asensio and D. Jornet, Global pseudodifferential operators of infinite order in classes of ultradifferentiable functions, Rev. R. Acad. Cienc. Exactas Fís. Nat. Ser. A Mat. RACSAM 113 (2019), no. 4, 3477-3512.

[7] A. T. Benjamin and J. J. Quinn, Proofs that really count, The Dolciani Mathematical Expositions, vol. 27, Mathematical Association of America, Washington, DC, 2003, The art of combinatorial proof.

[8] G. Björck, Linear partial differential operators and generalized distributions, Ark. Mat. 6 (1966), 351-407. 
[9] P. Boggiatto, E. Buzano, and L. Rodino, Global hypoellipticity and spectral theory, Mathematical Research, vol. 92, Akademie Verlag, Berlin, 1996.

[10] C. Boiti and D. Jornet, A characterization of the wave front set defined by the iterates of an operator with constant coefficients, Rev. R. Acad. Cienc. Exactas Fís. Nat. Ser. A Mat. RACSAM 111 (2017), no. 3, 891-919.

[11] A simple proof of Kotake-Narasimhan theorem in some classes of ultradifferentiable functions, J. Pseudo-Differ. Oper. Appl. 8 (2017), no. 2, 297-317.

[12] C. Boiti, D. Jornet, and J. Juan-Huguet, Wave front sets with respect to the iterates of an operator with constant coefficients, Abstr. Appl. Anal. (2014), Art. ID 438716, 17.

[13] C. Boiti, D. Jornet, and A. Oliaro, Regularity of partial differential operators in ultradifferentiable spaces and Wigner type transforms, J. Math. Anal. Appl. 446 (2017), no. 1, 920-944.

[14] , The Gabor wave front set in spaces of ultradifferentiable functions, Monatsh. Math. 188 (2019), no. 2, 199-246.

[15] , Real Paley-Wiener theorems in spaces of ultradifferentiable functions, J. Funct. Anal. 278 (2020), no. 4, 108348, 45.

[16] C. Boiti, D. Jornet, A. Oliaro, and G. Schindl, Nuclear global spaces of ultradifferentiable functions in the matrix weighted setting, Banach J. Math. Anal. 15 (2021), no. 1, 14.

[17] Nuclearity of rapidly decreasing ultradifferentiable functions and time-frequency analysis, Collect. Math. 72 (2021), no. 2, 423-442.

[18] J. Bonet, R. Meise, and S. N. Melikhov, A comparison of two different ways to define classes of ultradifferentiable functions, Bull. Belg. Math. Soc. Simon Stevin 14 (2007), no. 3, 425-444.

[19] R. W. Braun, An extension of Komatsu's second structure theorem for ultradistributions, J. Fac. Sci. Univ. Tokyo Sect. IA Math. 40 (1993), no. $2,411-417$.

[20] R. W. Braun, R. Meise, and B. A. Taylor, Ultradifferentiable functions and Fourier analysis, Results Math. 17 (1990), no. 3-4, 206-237.

[21] J. Bruna, On inverse-closed algebras of infinitely differentiable functions, Studia Math. 69 (1980/81), no. 1, 59-68. 
[22] M. Cappiello, Pseudodifferential parametrices of infinite order for $S G$ hyperbolic problems, Rend. Sem. Mat. Univ. Politec. Torino 61 (2003), no. 4, 411-441 (2004).

[23] _ Fourier integral operators of infinite order and applications to SG-hyperbolic equations, Tsukuba J. Math. 28 (2004), no. 2, 311-361.

[24] M. Cappiello, T. Gramchev, and L. Rodino, Gelfand-Shilov spaces, pseudo-differential operators and localization operators, Modern trends in pseudo-differential operators, Oper. Theory Adv. Appl., vol. 172, Birkhäuser, Basel, 2007, pp. 297-312.

[25] M. Cappiello, S. Pilipović, and B. Prangoski, Parametrices and hypoellipticity for pseudodifferential operators on spaces of tempered ultradistributions, J. Pseudo-Differ. Oper. Appl. 5 (2014), no. 4, 491-506.

[26] M. Cappiello and R. Schulz, Microlocal analysis of quasianalytic GelfandShilov type ultradistributions, Complex Var. Elliptic Equ. 61 (2016), no. 4, $538-561$.

[27] M. Cappiello and J. Toft, Pseudo-differential operators in a GelfandShilov setting, Math. Nachr. 290 (2017), no. 5-6, 738-755.

[28] J. Chazarain and A. Piriou, Introduction to the theory of linear partial differential equations, Studies in Mathematics and its Applications, vol. 14, North-Holland Publishing Co., Amsterdam-New York, 1982.

[29] E. Cordero and K. Gröchenig, Time-frequency analysis of localization operators, J. Funct. Anal. 205 (2003), no. 1, 107-131.

[30] J. J. Duistermaat and J. A. C. Kolk, Distributions, Cornerstones, Birkhäuser Boston, Inc., Boston, MA, 2010, Theory and applications.

[31] C. Fernández and A. Galbis, Superposition in classes of ultradifferentiable functions, Publ. Res. Inst. Math. Sci. 42 (2006), no. 2, 399-419.

[32] C. Fernández, A. Galbis, and D. Jornet, $\omega$-hypoelliptic differential operators of constant strength, J. Math. Anal. Appl. 297 (2004), no. 2, 561-576, Special issue dedicated to John Horváth.

[33] _ Pseudodifferential operators on non-quasianalytic classes of Beurling type, Studia Math. 167 (2005), no. 2, 99-131.

[34] _ Pseudodifferential operators of Beurling type and the wave front set, J. Math. Anal. Appl. 340 (2008), no. 2, 1153-1170. 
[35] U. Franken, Weight functions for classes of ultradifferentiable functions, Results Math. 25 (1994), no. 1-2, 50-53.

[36] M.C. Gómez Collado, Ultradistribuciones casi-periodicas y operadores de convolucion elipticos, ProQuest LLC, Ann Arbor, MI, 2000, Thesis (Ph.D.)-Universitat de Valencia (Spain).

[37] A. Grigis and J. Sjöstrand, Microlocal analysis for differential operators, London Mathematical Society Lecture Note Series, vol. 196, Cambridge University Press, Cambridge, 1994, An introduction.

[38] K. Gröchenig, Foundations of time-frequency analysis, Applied and Numerical Harmonic Analysis, Birkhäuser Boston, Inc., Boston, MA, 2001.

[39] K. Gröchenig and G. Zimmermann, Spaces of test functions via the STFT, J. Funct. Spaces Appl. 2 (2004), no. 1, 25-53.

[40] G. Grubb, Distributions and operators, Graduate Texts in Mathematics, vol. 252, Springer, New York, 2009.

[41] S. Hashimoto, Y. Morimoto, and T. Matsuzawa, Opérateurs pseudodifférentiels et classes de Gevrey, Comm. Partial Differential Equations 8 (1983), no. 12, 1277-1289.

[42] T. Heinrich and R. Meise, A support theorem for quasianalytic functionals, Math. Nachr. 280 (2007), no. 4, 364-387.

[43] L. Hörmander, Pseudo-differential operators, Comm. Pure Appl. Math. 18 (1965), 501-517.

[44] _ Fourier integral operators. I, Acta Math. 127 (1971), no. 1-2, $79-183$.

[45] _ Quadratic hyperbolic operators, Microlocal analysis and applications (Montecatini Terme, 1989), Lecture Notes in Math., vol. 1495, Springer, Berlin, 1991, pp. 118-160.

[46] V. Iftimie, Opérateurs hypoelliptiques dans des espaces de Gevrey, Bull. Math. Soc. Sci. Math. R. S. Roumanie (N.S.) 27(75) (1983), no. 4, 317333.

[47] D. Jornet Casanova, Operadores pseudodiferenciales en clases no casianaliticas de tipo Beurling, Ph.D. thesis, Universitat Politècnica de València, 2004, 10.4995/Thesis/10251/54953. 
[48] J. J. Kohn and L. Nirenberg, An algebra of pseudo-differential operators, Comm. Pure Appl. Math. 18 (1965), 269-305.

[49] H. Komatsu, Ultradistributions. I. Structure theorems and a characterization, J. Fac. Sci. Univ. Tokyo Sect. IA Math. 20 (1973), 25-105.

[50] H. Kumano-go, Pseudodifferential operators, MIT Press, Cambridge, Mass.-London, 1981.

[51] M. Langenbruch, Continuation of Gevrey regularity for solutions of partial differential operators, Functional analysis (Trier, 1994), de Gruyter, Berlin, 1996, pp. 249-280.

[52] M. Mascarello and L. Rodino, Partial differential equations with multiple characteristics, Mathematical Topics, vol. 13, Akademie Verlag, Berlin, 1997.

[53] C. Mele and A. Oliaro, Regularity of global solutions of partial differential equations in non isotropic ultradifferentiable spaces via time-frequency methods, J. Differential Equations 286 (2021), 821-855.

[54] S. Nakamura, Propagation of the homogeneous wave front set for Schrödinger equations, Duke Math. J. 126 (2005), no. 2, 349-367.

[55] F. Nicola and L. Rodino, Global pseudo-differential calculus on Euclidean spaces, Pseudo-Differential Operators. Theory and Applications, vol. 4, Birkhäuser Verlag, Basel, 2010.

[56] J. Peetre, Concave majorants of positive functions, Acta Math. Acad. Sci. Hungar. 21 (1970), 327-333.

[57] H. J. Petzsche and D. Vogt, Almost analytic extension of ultradifferentiable functions and the boundary values of holomorphic functions, Math. Ann. 267 (1984), no. 1, 17-35.

[58] B. Prangoski, Pseudodifferential operators of infinite order in spaces of tempered ultradistributions, J. Pseudo-Differ. Oper. Appl. 4 (2013), no. 4, 495-549.

[59] A. Rainer and G. Schindl, Composition in ultradifferentiable classes, Studia Math. 224 (2014), no. 2, 97-131.

[60] L. Rodino, Linear partial differential operators in Gevrey spaces, World Scientific Publishing Co., Inc., River Edge, NJ, 1993. 
[61] L. Rodino and P. Wahlberg, The Gabor wave front set, Monatsh. Math. 173 (2014), no. 4, 625-655.

[62] V. Scheidemann, Introduction to complex analysis in several variables, Birkhäuser Verlag, Basel, 2005.

[63] R. Schulz and P. Wahlberg, Equality of the homogeneous and the Gabor wave front set, Comm. Partial Differential Equations 42 (2017), no. 5, 703-730.

[64] M. A. Shubin, Pseudodifferential operators and spectral theory, second ed., Springer-Verlag, Berlin, 2001.

[65] L. Zanghirati, Pseudodifferential operators of infinite order and Gevrey classes, Ann. Univ. Ferrara Sez. VII (N.S.) 31 (1985), 197-219 (1986). 
